Maria do Socorro Martins Sampaio

\title{
ANÁLISE NÃO LINEAR GEOMÉTRICA DE CASCAS LAMINADAS REFORÇADAS COM FIBRAS
}

\author{
VERSÃO CORRIGIDA \\ A versão original encontra-se na Escola de Engenharia de São Carlos. \\ Tese apresentada à Escola de Engenharia de São \\ Carlos da Universidade de São Paulo, como \\ parte dos requisitos para obtenção do título de \\ Doutor em Ciências - Engenharia Civil - Área de \\ Concentração: Engenharia de Estruturas.
}

Orientador: Prof. Tit. Humberto Breves Coda

São Carlos

2014 
AUTORIZO A REPRODUÇÃO TOTAL OU PARCIAL DESTE TRABALHO, POR QUALQUER MEIO CONVENCIONAL OU ELETRÔNICO, PARA FINS DE ESTUDO E PESQUISA, DESDE QUE CITADA A FONTE.

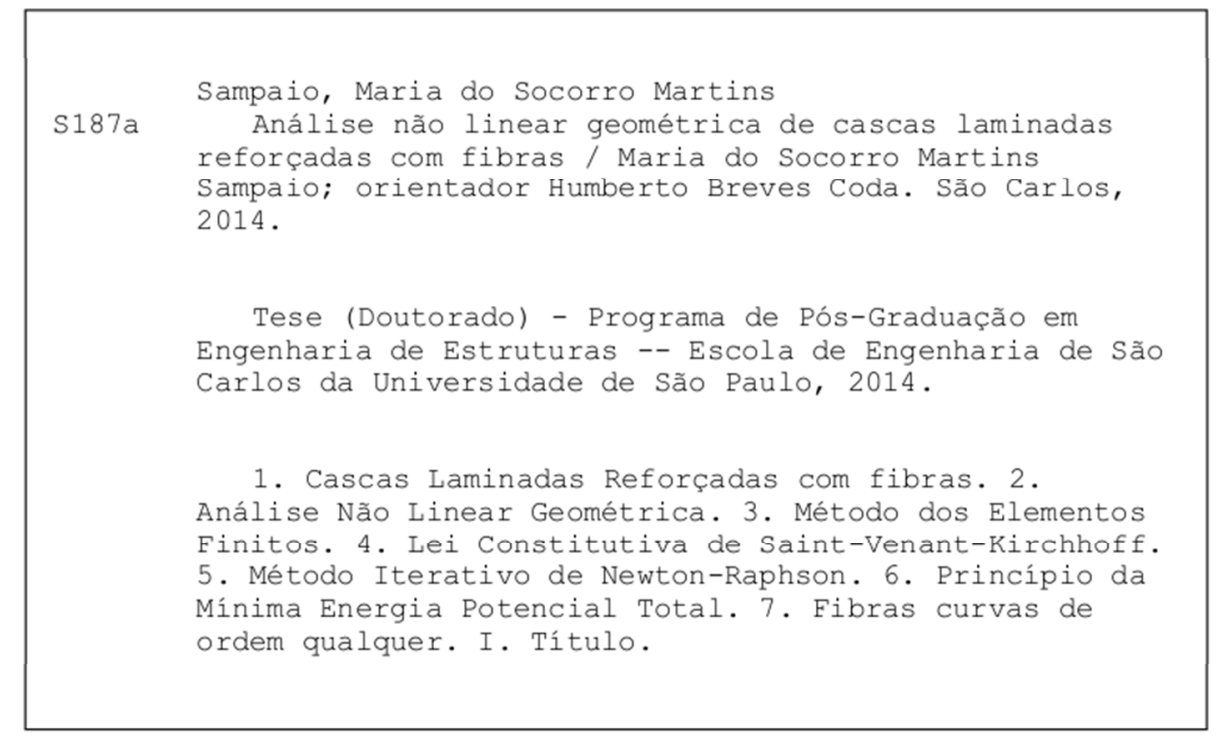


FOLHA DE JULGAMENTO

Candidata: Engenheira MARIA DO SOCORRO MARTINS SAMPAIO.

Título da tese: "Análise não linear geométrica de cascas laminadas reforçadas com fibras".

Data da defesa: 03/02/2014

\section{Comissão Julgadora:}

Resultado:

Prof. Titular Humberto Breves Coda (Orientador)

(Escola de Engenharia de São Carlos/EESC)

Apnorada

Prof. Associado Márcio Roberto Silva Corrêa

APROVADA

(Escola de Engenharia de São Carlos/EESC)

Prof. Dr. Rodrigo Ribeiro Paccola

fProvarA

(Escola de Engenharia de São Carlos/EESC)

Profa. Dra. Larissa Driemeier

APROVADA

(Escola Politécnica/USP)

Prof. Dr. José Benaque Rubert

APROVADA

(Universidade Federal de São Carlos/UFSCar)

Coordenadora do Programa de Pós-Graduação em Engenharia Civil (Engenharia de Estruturas):

Profa. Associada Ana Lucia Homce de Cresce El Debs

Presidente da Comissão de Pós-Graduação:

Prof. Titular Denis Vinicius Coury 

Para

Izabel, Sampaio,

Diego e Lorena 

"Professores nunca morrem.

Vivem em sua memória para sempre.

Eles estavam lá quando você chegou;

eles ficaram lá quando você foi embora.

Como acessórios.

Às vezes lhe ensinavam alguma coisa,

mas nem sempre.

E você nunca chegava a conhecê-los realmente

nem eles a você.

Ainda assim, por algum tempo, você acreditava neles.

E, se tivesse sorte,

talvez um deles acreditasse em você".

Extraído de Anos Incriveis

Em homenagem ao

Prof. Wilson Sérgio Venturini

(In memoriam) 



\section{AGRADECIMENTOS}

Ao professor Tit. Humberto Breves Coda gostaria de agradecer a orientação, dedicação, disponibilidade, generosidade e paciência demonstradas durante todo $o$ desenvolvimento desta pesquisa.

Ao professor Dr. Rodrigo Ribeiro Paccola agradeço as sugestões e contribuições feitas em diversas etapas deste trabalho.

Aos funcionários do LIMC (Laboratório de Informática e Mecânica Computacional "Wilson Sérgio Venturini"), Masaki Kawabata Neto, Dorival Piedade Neto, Roberto Fagá Júnior e Raphael Mulinari pelo suporte prestado durante o desenvolvimento desta pesquisa.

Aos professores e funcionários do Departamento de Engenharia de Estruturas (SET/EESC/USP). Em especial, à secretária de Pós-graduação Rosi Aparecida Jordão Rodrigues, à Maria Nadir Minatel e à Melina Benatti Ostini.

Aos funcionários do serviço de biblioteca (EESC/USP) pelos excelentes serviços prestados e pela excelente infraestrutura disponibilizada facilitando o acesso aos artigos e referências bibliográficas necessárias para o desenvolvimento desta pesquisa.

Aos amigos, Fabio Carlos da Rocha e Aref Kalilo Lima Kzam, pelas experiências e conhecimentos compartilhados ao longo destes anos.

Ao Departamento de Engenharia de Estruturas da Escola de Engenharia de São Carlos da Universidade de São Paulo (SET/EESC/USP) pela disponibilização das instalações e recursos computacionais para o desenvolvimento desta pesquisa.

À Coordenação de Aperfeiçoamento de Pessoal de Nível Superior (CAPES) pela bolsa de estudos concedida.

À Fundação de Amparo à Pesquisa do Estado do Amazonas (FAPEAM), à Secretaria de Estado de Ciência, Tecnologia e Inovação (SECTI) e ao Governo do Estado do Amazonas pela bolsa de estudos concedida. 

Onde se achará a sabedoria, e onde está o lugar da inteligência? O homem não conhece o seu valor, e nem ela se acha na terra dos viventes.

O abismo diz: Não está em mim; e o mar diz: Ela não está comigo.

Com ela não se pode comparar o ouro nem o cristal nem se trocará por joia de ouro fino. Não se fará menção de coral nem de pérolas; porque o valor da sabedoria é melhor que o dos rubis.

Não se lhe igualará o topázio da Etiópia, nem se pode avaliar por ouro puro.

Donde, pois, vem a sabedoria, e onde está o lugar da inteligência? Pois está encoberta aos olhos de todo o vivente, e oculta às aves do céu. A perdição e a morte dizem: Ouvimos com os nossos ouvidos a sua fama.

Deus entende o seu caminho, e ele sabe o seu lugar.

Porque ele vê as extremidades da terra; e vê tudo o que há debaixo dos céus.

Quando deu peso ao vento, e tomou a medida das águas;

Quando prescreveu leis para a chuva e caminho para o relâmpago dos trovões;

Então a viu e relatou;

estabeleceu- $a$, e também a esquadrinhou.

E disse ao homem:

Eis que o temor do Senhor é a sabedoria, e apartar-se do mal é a inteligência. 



\section{RESUMO}

SAMPAIO, M. S. M. Análise não linear geométrica de cascas laminadas reforçadas com fibras. 2014. 190 f. Tese (Doutorado) - Escola de Engenharia de São Carlos, Universidade de São Paulo, São Carlos, 2014.

Em geral, as formulações disponíveis na literatura para a análise de cascas laminadas reforçadas com fibras substituem o meio original heterogêneo por um homogêneo equivalente, que dificulta a identificação das tensões fibra-matriz, ou requerem que a malha de elementos finitos seja disposta de modo que os nós dos elementos finitos de fibra coincidam com os nós dos elementos finitos de casca, que é uma exigência bastante restritiva e que aumenta o número de graus de liberdade do sistema de equações resultante. Neste sentido, o objetivo geral desta tese consiste em desenvolver uma formulação para a inclusão de fibras longas e curtas aleatórias nas diversas lâminas de cascas laminadas anisotrópicas com não linearidade geométrica utilizando o método dos elementos finitos sem aumentar o número de graus de liberdade do sistema de equações resultante e sem a necessidade de coincidência de nós na discretização das fibras e da matriz. Nesta formulação, o elemento finito triangular de casca laminada utilizado para discretizar a matriz possui dez nós e sete graus de liberdade por nó, sendo três translações, três componentes do vetor generalizado e a taxa de variação linear da deformação ao longo da espessura. As fibras curvas, curtas aleatórias ou longas, são introduzidas, em qualquer camada do laminado, por meio de relações cinemáticas que garantem sua aderência à matriz sem a introdução de novos graus de liberdade no sistema de equações resultante. Para discretizá-las são utilizados elementos finitos unidimensionais de ordem qualquer com três graus de liberdade por nó e que consideram consistentemente a não linearidade geométrica. Todas as grandezas envolvidas são escritas em relação à configuração inicial do corpo, caracterizando a descrição Lagrangeana total ou material do movimento. Para modelar o comportamento do material adota-se a Lei Constitutiva de Saint-Venant-Kirchhoff que relaciona de forma linear o tensor de tensões de Piolla-Kirchhoff de segunda espécie e o tensor de deformações de GreenLagrange. O equilíbrio é encontrado a partir do Princípio da Mínima Energia Potencial Total e o sistema não linear de equações resultante é resolvido utilizando-se o procedimento iterativo de Newton-Raphson. As ações externas podem ser introduzidas ao sistema de forma total ou incremental e a contribuição das fibras para a energia do sistema é adicionada na matriz global do problema. Os exemplos numéricos testados validam e demonstram as potencialidades da formulação proposta.

Palavras-chave: Cascas laminadas reforçadas com fibras. Análise não linear geométrica. Método dos Elementos Finitos. Lei Constitutiva de Saint-Venant-Kirchhoff. Método Iterativo de Newton-Raphson. Princípio da Mínima Energia Potencial. Fibras curvas de ordem qualquer. 



\section{ABSTRACT}

SAMPAIO, M. S. M. Geometrically nonlinear analysis of fiber reinforced laminated shells. 2014. 190 f. Tese (Doutorado) - Escola de Engenharia de São Carlos, Universidade de São Paulo, São Carlos, 2014.

In general, the Finite Element (FE) formulations available in the literature for the analysis of fibre reinforced laminated shells replace the original heterogeneous medium by an equivalent homogeneous one, which makes difficult the identification of fiber-matrix stress distribution, or require that the finite element mesh is arranged in a way that the fibre finite element nodes coincide with the shell finite element ones, which is a very restrictive requirement and increases the number of degrees of freedom of the resulting system of equations. In this sense, the objective of this thesis is to develop a formulation for the inclusion of long and random short fibres in any layer of FE laminated anisotropic shells developing large displacement and rotations without increasing the number of degrees of freedom and the necessity of matching nodes in the discretization of the fibre and the matrix. In this formulation, the triangular laminated shell finite element used to discretize the matrix has ten nodes and seven degrees of freedom per node, that are, three translations, three components of a generalized vector and the linear rate of strain variation along the thickness. The curved fibres, long or random short, are introduced in any layer of the laminate shell by means of kinematic relation to ensure its adherence to the matrix without introducing new degrees of freedom in the resulting system of equations. To discretize them, any order one-dimensional finite elements with three degrees of freedom per node are used. These fibres elements are consistently considered by Geometric nonlinearity. All involved variables are written with respect to the initial configuration of the body, characterizing the Total Lagrangian description. To model the behavior of the material we use the Saint-Venant-Kirchhoff Constitutive Law that relates linearly the second Piolla-Kirchhoff stress tensor and Green-Lagrange strain tensor. The equilibrium is achieved from the Principle of Minimum Potential Energy and the non-linear system of equations is solved by the Newton-Raphson iterative procedure. External loads may be introduced to the system by one or various steps and the contribution of fibres to the energy of the system is added to the global matrix of the problem. The numerical examples validate and demonstrate the potential of the proposed formulation.

Keywords: Fibre reinforced laminated shells. Geometric nonlinear analysis. Finite Element Method. Saint-Venant-Kirchhoff Constitutive Law. Newton-Raphson iterative procedure. Principle of minimum potential energy. Any order curved fibres. 



\section{LISTA DE FIGURAS}

Figura 1 - Classificação dos materiais compósitos reforçados com fibras.

Figura 2 - Características da solução de um problema não linear. Fonte: (YANG; SHIEH, 1990).

Figura 3 - Alguns elementos planos para a análise de cascas. Fonte: (YANG; SAIGAL; LIAW, 1990).

Figura 4 - Comparação de dois conceitos básicos utilizados na obtenção de elementos de casca. (a) elementos degenerados e (b) elementos bidimensionais. Fonte: (GILEWSKI; RADWAFLSKA, 1991).

Figura 5 - Configuração de um corpo em equilíbrio

Figura 6 - Representação gráfica do Método de Newton-Raphson.

Figura 7 - Fluxograma com o algoritmo para implementação computacional do Método de

Newton-Raphson. 52

Figura 8 - Mapeamento do elemento bidimensional nas configurações inicial e final. 57

Figura 9 - Elemento finito de barra simples nas configurações inicial $\Omega^{0}$ e final $\Omega$ 61

Figura 10 - Mapeamento do elemento de fibra curvo de ordem qualquer nas configurações inicial e final.

Figura 11 - Arranjos das fibras na matriz. Fonte: (DANIEL; ISHAI, 2006)..........................72

Figura 12 - Arranjo das fibras no domínio bidimensional.................................................73

Figura 13 - Sub-rotina principal para contribuição das fibras na energia do sistema..............75 Figura 14 - Programa principal para a análise de domínios bidimensionais reforçados com fibras.

Figura 15 - Viga simplesmente engastada reforçada com fibras.

Figura 16 - Configuração deformada da viga homogênea em $\mathrm{cm}$ : (a) presente formulação linear; (b) barra geral 3D - linear; (c) presente formulação - não linear; (d) barra geral 3D não linear.

Figura 17 - Configuração deformada da viga com fibras: (a) presente formulação - linear; (b) barra geral 3D - linear; (c) presente formulação - não linear; (d) barra geral 3D - não linear.

Figura 18 - Força normal na fibra superior.

Figura 19 - Domínio bidimensional hipotético reforçado com fibra.

Figura 20 - Matriz com aproximação cúbica e fibras com aproximação variando de linear ao

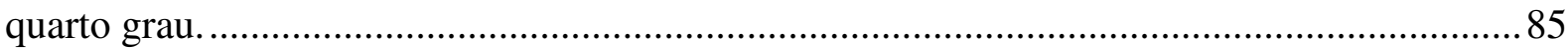

Figura 21 - Chapa engastada reforçada com fibras........................................................ 87

Figura 22 - Deslocamentos horizontais e verticais da chapa da Figura 21........................... 89

Figura 23 - Atualização das posições das fibras. .................................................................. 89

Figura 24 - Deslocamentos horizontais e verticais da chapa enrijecida com fibras aleatórias.89

Figura 25 - Deslocamentos horizontais e verticais da chapa da Figura 21............................90

Figura 26 - Atualização das posições das fibras..................................................................90 
Figura 27 - Deslocamentos horizontais e verticais da chapa enrijecida com fibras aleatórias 90 $\begin{array}{llllll}\text { Figura } & 28 & - & \text { Chopped } & \text { strand } & \text { Fonte: }\end{array}$ http://www.easycomposites.co.uk/products/chopped-strand-mat/powder-bound-choppedstrand-mat-300g-per-metre.aspx. Acesso em 01/11/2013 .................................................... 91

Figura 29 - Chapa quadrada reforçada com fibra e deslocamento imposto. ........................... 92

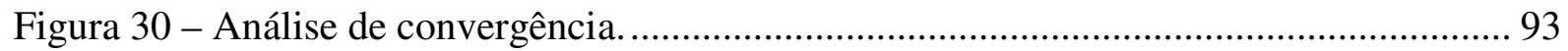

Figura 31 - Análise de dependência da discretização da fibra. ............................................. 93

Figura 32 - Resultados de Radtke, Simone e Sluys (2010a) para a análise de convergência da força de reação devido à discretização da matriz. Fonte: Radtke, Simone e Sluys (2010a).... 95

Figura 33 - Chapa reforçado com fibras com extremidades curvas em (cm)........................ 97

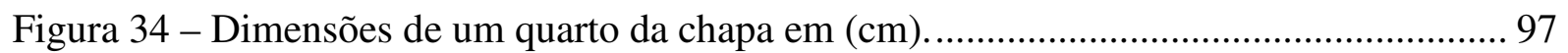

Figura 35 - Malha 1: 1000 elementos triangulares cúbicos totalizando 9362 graus de

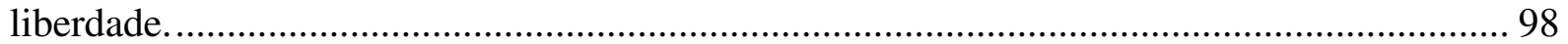

Figura 36 - Malha 2: 4000 elementos triangulares cúbicos totalizando 36722 graus de

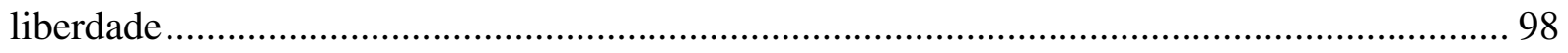

Figura 37 - Malha 3: 9000 elementos triangulares cúbicos totalizando 82082 graus de

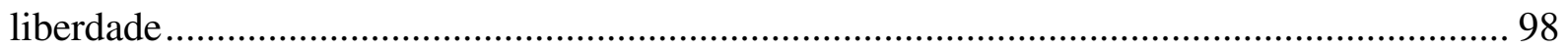

Figura 38 - Componente $\hat{\Gamma}_{11}$ da Tensão de Cauchy para as diferentes discretizações adotadas

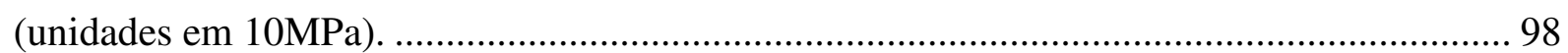

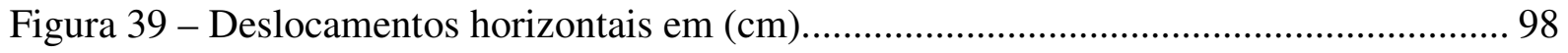

Figura 40 - Efeito da deformação de uma matriz com baixo módulo de elasticidade na fibra.

Figura 41 - Distribuição de tensão normal na fibra e de cisalhamento na interface fibra-

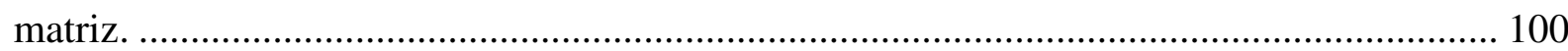

Figura 42 - Vetores associados a um nó de um trecho de fibra curvo. .................................. 101

Figura 43 - Equilíbrio diferencial na direção da fibra. ..................................................... 101

Figura 44 - Sub-rotina principal que gerencia a determinação da força normal nas fibras... 103 Figura 45 - Sub-rotina principal que gerencia a determinação da tensão de aderência nas

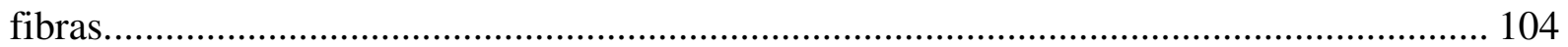

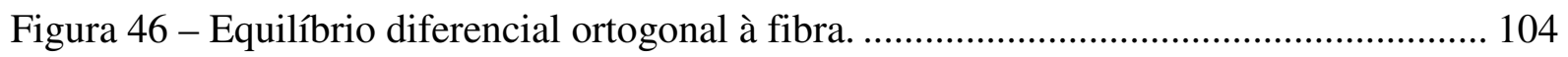

Figura 47 - Sub-rotina principal que gerencia a determinação da tensão normal de contato nas

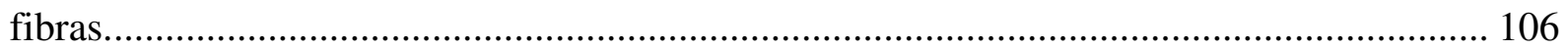

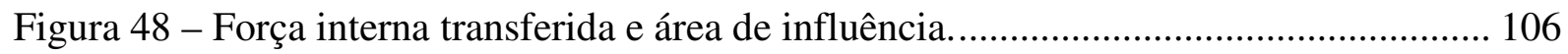

Figura 49 - Sub-rotina principal que gerencia a obtenção da tensão normal de contato e tensão de aderência nas fibras pela formulação média. ................................................................... 107

Figura 50 - Barra reforçada submetida a deslocamento controlado (unidades em cm). ....... 108 Figura 51 - Malha igualmente espaçada: 864 elementos triangulares e 80 elementos de fibra -

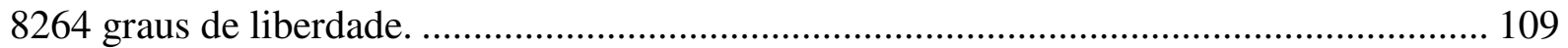

Figura 52 - Força normal e tensão de aderência: formulação diferencial. ............................ 109

Figura 53 - Tensão de aderência: formulação média. ........................................................... 109

Figura 54 - Malha distorcida: 864 elementos triangulares e 80 elementos de fibra - 8246 graus

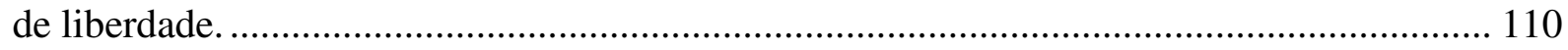

Figura 55 - Força normal na fibra e tensão de aderência: formulação diferencial................. 110 
Figura 56 - Tensão de aderência: formulação média.

Figura 57 - Barra reforçada com fibras curvas (unidades em $\mathrm{cm}$ )..................................... 111

Figura 58 - Malha distorcida: 225x45 elementos triangulares cúbicos - 183872 graus de liberdade.

Figura 59 - Malha distorcida: 150x30 elementos triangulares cúbicos - 82082 graus de liberdade.

Figura 60 - Força normal - kN.

Figura 61 - Tensão de aderência $-\mathrm{kN} / \mathrm{cm}^{2}$

Figura 62 - Tensão Normal de contato $-\mathrm{kN} / \mathrm{cm}^{2}$.

Figura 63 - Mapeamento da superfície média do elemento finito de casca. Fonte: (CODA; PACCOLA, 2008).

Figura 64 - Mapeamento dos pontos fora da superfície média. Fonte: (PASCON, 2008)... 117

Figura 65 - Mapeamento dos pontos fora da superfície média do elemento de casca. Fonte: (CODA; PACCOLA, 2008)

Figura 66 - Casca laminada.

Figura 67 - Cinemática para cascas laminadas.

Figura 68 - Discretização fibra-matriz da superfície de referência do laminado.

Figura 69 - Viga engastada reforçada com fibras.

Figura 70 - Geometria da seção transversal: (a) original, (b) laminada equivalente.

Figura 71 - Deslocamentos transversais da viga: (a) barra geral 3D; (b) domínio 2D; (c) placa laminada equivalente; (d) placa com fibras.

Figura 72 - Deslocamento transversal da viga em $(\mathrm{cm})$.

Figura 73 - Placa quadrada simplesmente apoiada reforçada com fibras: (a) reforçada em uma direção; (b) reforçada em duas direções.

Figura 74 - Discretização para um quarto do problema: (a) matriz; (b) fibras para placa reforçada em uma direção; (c) fibras para placa reforçada em duas direções.

Figura 75 - Deslocamentos transversais para um quarto do problema: (a) placa reforçada em uma direção laminada equivalente; (b) placa reforçada em uma direção com fibras; (c) placa reforçada em duas direções laminada equivalente; (d) placa reforçada em duas direções com fibras.

Figura 76 - Deslocamento transversal máximo para diferentes valores de módulo de elasticidade, coeficiente de Poisson e módulo de elasticidade transversal.....

Figura 77 - Cilindro laminado comprimido. Fonte: (SANSOUR; KOLLMANN, 2000). ....138

Figura 78 - Detalhe de 1/4 da seção transversal do cilindro laminado.

Figura 79 - Discretização da superfície de referência da matriz: 20x20 - 800 elementos com aproximação cúbica para as variáveis totalizando 3721 nós e 26047 graus de liberdade...... 139 Figura 80 - Arranjos fibra-matriz considerados: $0^{\circ}$ corresponde à direção longitudinal e $90^{\circ}$ à direção circunferencial.

Figura 81 - Discretização das fibras para os diferentes arranjos testados.

Figura 82 - Deslocamentos na direção de P de 1/8 do cilindro para os diferentes arranjos testados: (a) laminada equivalente; (b) laminada com fibras.

Figura 83 - Configurações finais deformadas de 1/8 do cilindro laminado: (a) sem fibras; (b) com 3568 fibras aleatórias; (c) com 1854 fibras aleatórias; (d) com 924 fibras aleatórias.... 146 
Figura 84 - Trajetória de equilíbrio do ponto de aplicação da carga $\mathrm{P}$ para os diferentes arranjos considerados.

Figura 85 - Trajetória de equilíbrio do ponto de aplicação da carga P para a casca laminada

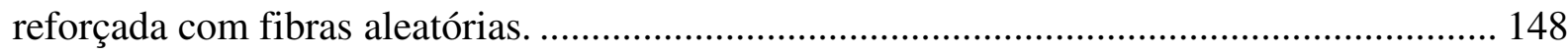

Figura 86 - Comparação entre as trajetórias de equilíbrio para os arranjos considerados. ... 149 Figura 87 - Configuração final de $1 / 8$ do cilindro em grandes deslocamentos para os diferentes arranjos testados: (a) laminada equivalente; (b) laminada com fibras; (c) laminada com fibras aleatórias.

Figura 88 - Vista ampliada da configuração final deformada de 1/8 do cilindro reforçado com fibras: arranjo $0^{\circ} / 0^{\circ} / 0^{\circ}$.

Figura 89 - Vista ampliada da configuração final deformada de 1/8 do cilindro reforçado com fibras: arranjo $0^{\circ} / 90^{\circ} / 0^{\circ}$.

Figura 90 - Vista ampliada da configuração deformada de $1 / 8$ do cilindro reforçado com fibras aleatórias.

Figura 91 - Outras vistas ampliadas da configuração final deformada de $1 / 8$ do cilindro reforçado com fibras aleatórias.

Figura 92 - Matriz com aproximação linear e fibras com aproximação variando de linear ao quarto grau.

Figura 93 - Matriz com aproximação quadrática e fibras com aproximação variando de linear ao quarto grau.

Figura 94 - Matriz com aproximação do quarto grau e fibras com aproximação variando de

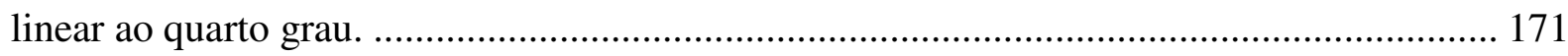

Figura 95 - Estrutura do programa para geração de fibras. ................................................ 173

Figura 96 - Programa principal para gerenciamento das sub-rotinas de geração das fibras. 174

Figura 97 - Arquivo de entrada de dados para a geração das fibras..................................... 177

Figura 98 - Arquivo de entrada de dados da superfície de referência.................................. 178

Figura 99 - Sub-rotina para geração das fibras aleatórias. ................................................ 179

Figura 100 - Arquivo de saída com dados das fibras. ......................................................... 180

Figura 101 - Arquivo de saída com informações das lâminas. ........................................... 181

Figura 102 - Discretização obtida como o ACADVIEW: (a) superfície de referência; (b)

fibras; (c) superfície de referência e fibras........................................................................ 182

Figura 103 - Coordenas homogêneas do elemento finito triangular. (Fonte: ASSAN, 2003).

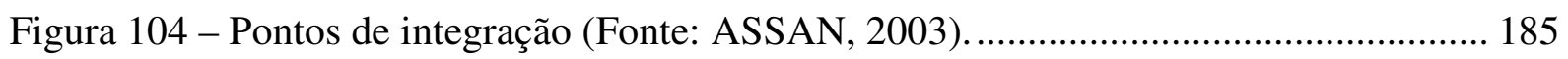

Figura 105 - PPonto P inserido em um elemento finito triangular......................................... 187

Figura 106 - Elemento finito triangular com dez nós.................................................... 189 


\section{LISTA DE TABELAS}

Tabela 1 - Deslocamentos máximos na extremidade livre da viga para os diferentes casos analisados em $(\mathrm{cm})$

Tabela 2 - Valores analíticos e numéricos de força normal para pontos selecionados em $(\mathrm{N})$.

Tabela 3 - Matriz com aproximação cúbica e fibras com aproximação variando de linear ao

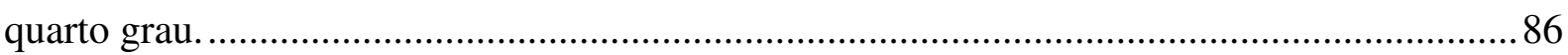
Tabela 4 - Deslocamentos máximos da chapa para as diferentes análises realizadas em $(\mathrm{cm})$.

Tabela 5 - Dados referentes à análise de convergência devido à discretização da malha da matriz.

Tabela 6 - Dados referentes à análise da influência da discretização das fibras na solução....94 Tabela 7 - Valores numéricos para o deslocamento transversal máximo em $(\mathrm{cm})$. .............. 133 Tabela 8 - Valores numéricos para o deslocamento transversal máximo em $(\mathrm{m})$................. 136 Tabela 9 - Dados utilizados para a modelagem do problema laminado equivalente com (E) $\times 10^{6}$ e $(\mathrm{G}) \times 10^{6}$.

Tabela 10 - Dados utilizados para a modelagem do problema com fibras com (E)x $10^{6}$ e (G)x $10^{6}$

Tabela 11 - Número total de nós de fibra longa para o arranjo com duas camadas de fibra por lâmina.

Tabela 12 - Deslocamento total na direção de P para o ponto de aplicação da carga P. ....... 142

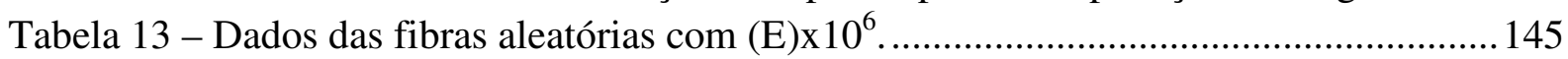

Tabela 14 - Número total de nós de fibras aleatórias........................................................ 145

Tabela 15 - Dados da matriz para análise do domínio com fibras aleatórias. ........................ 145

Tabela 16 - Deslocamentos na direção de P para o ponto de aplicação da carga $\mathrm{P}$ do cilindro reforçado com fibras aleatórias.

Tabela 17 - Matriz com aproximação linear e fibras com aproximação variando de linear ao quarto grau.

Tabela 18 - Matriz com aproximação quadrática e fibras com aproximação variando de linear ao quarto grau.

Tabela 19 - Matriz com aproximação do quarto grau e fibras com aproximação variando de linear ao quarto grau.

Tabela 20 - Pontos e pesos de Hammer (Fonte: ASSAN, 2003). 186 



\section{LISTA DE SÍMBOLOS}

Os significados dos símbolos são descritos à medida que aparecem no texto.

Em alguns casos as equações são descritas em notação intrínseca e em outros em termos das suas componentes (notação indicial), quando pareceu ser mais natural utilizar uma ou outra.

Observa-se apenas que para fazer distinção entre os meios considerados foi utilizado o padrão relacionado abaixo:

(ڤ) para o elemento bidimensional de ordem qualquer;

(•) para o elemento de fibra simples;

(ש) para o elemento de fibra curvo de ordem qualquer;

(•) para o elemento de casca. 



\section{SUMÁRIO}

1. INTRODUÇÃ

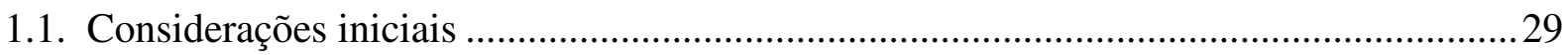

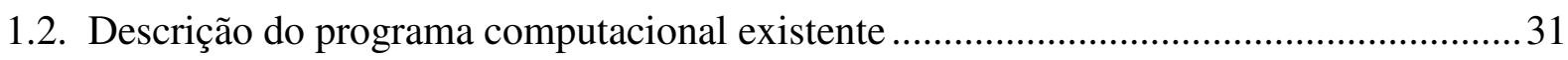

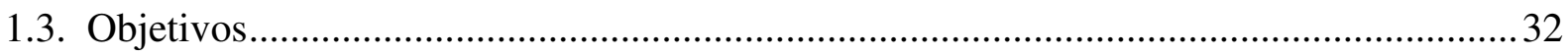

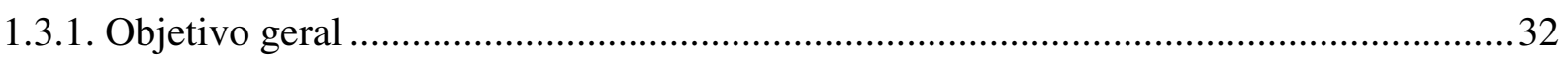

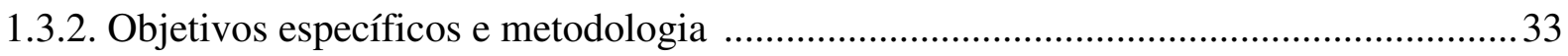

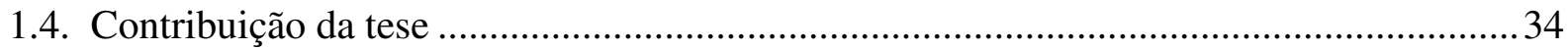

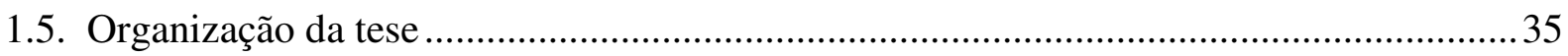

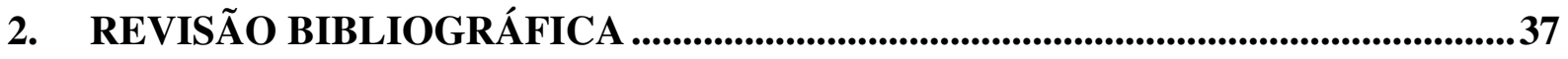

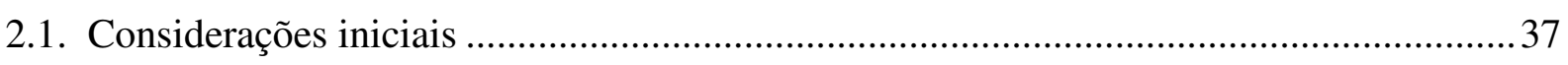

2.2. Dos primeiros elementos de casca até a sua aplicação a laminados nos dias atuais .........39

3. O PROBLEMA DE EQUILÍBRIO NÃO LINEAR GEOMÉTRICO.........................47

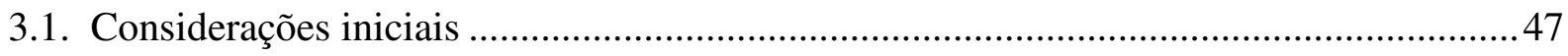

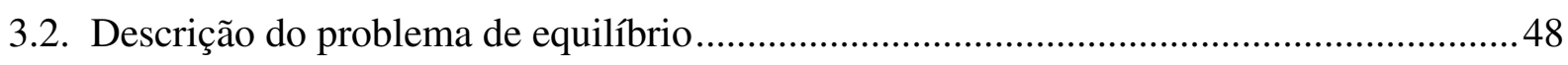

4. DOMÍNIOS BIDIMENSIONAIS REFORÇADOS COM FIBRAS ........................55

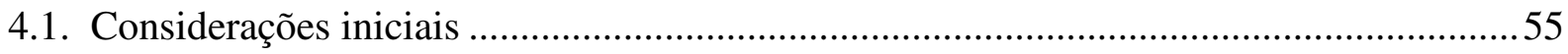

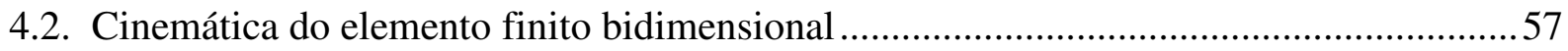

4.2.1. Energia específica de deformação do elemento bidimensional......................................59

4.2.2. Energia de deformação do elemento bidimensional ....................................................60

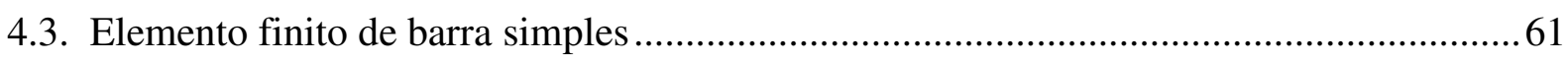

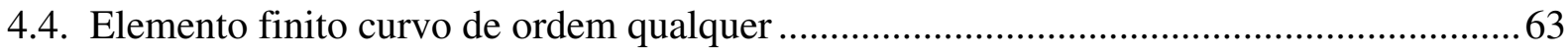

4.4.1.Cinemátca do elemento finito curvo de ordem qualquer............................................. 63

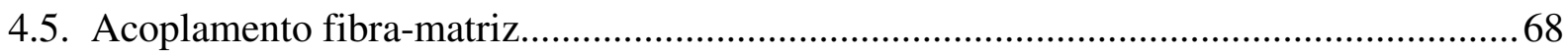

4.5.1. Energia de deformação de domínios elásticos bidimensionais reforçados com fibras força interna

4.5.2. Energia de deformação de domínios elásticos bidimensionais reforçados com fibras -

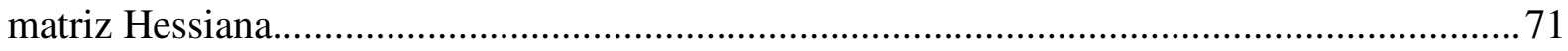

4.6. Procedimento matricial para o acoplamente fibra-matriz............................................ 72

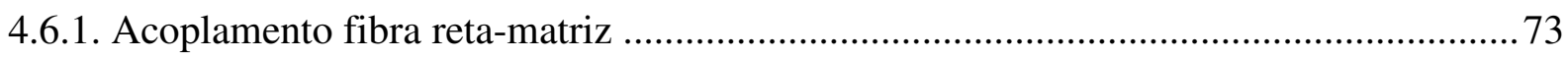


4.6.2. Acoplamento fibra curva-matriz

4.7. Conectividade nó de fibra ao elemento bidimensional ................................................. 75

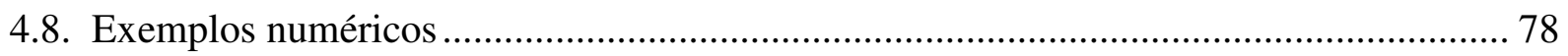

4.8.1. Exemplo 1: Viga reforçada com fibras submetida a grandes deslocamentos ................ 78

4.8.2. Exemplo 2: Conformidade do mapeamento fibra-matriz ........................................... 83

4.8.3. Exemplo 3: Chapa reforçada com fibras aleatórias ....................................................... 87

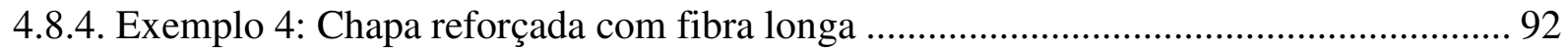

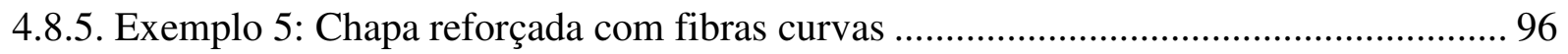

5. ANÁLISE DE TENSÃO FIBRA-MATRIZ EM MEIOS BIDIMENSIONAIS ....... 99

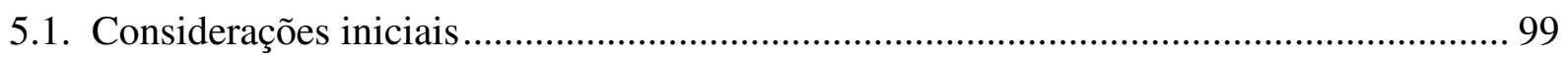

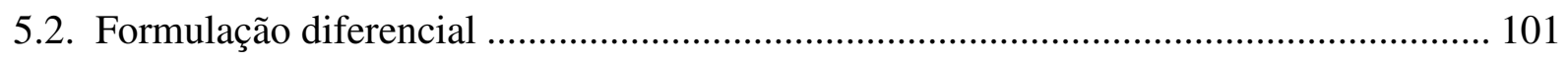

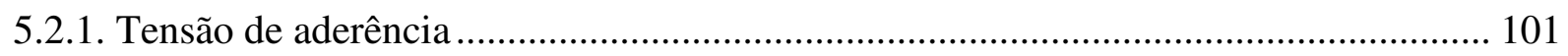

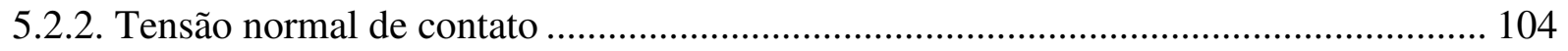

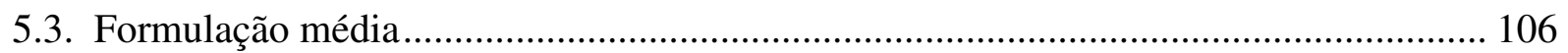

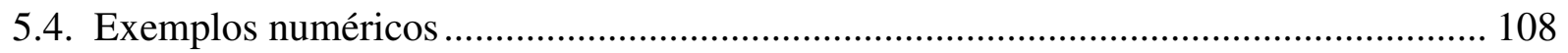

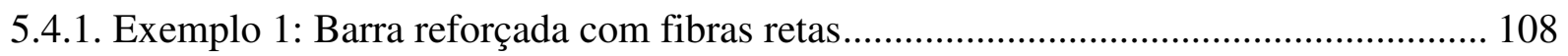

5.4.2. Exemplo 2: Barra reforçada com fibras curvas........................................................... 111

6. CASCAS LAMINADAS REFORÇADAS COM FIBRAS......................................... 115

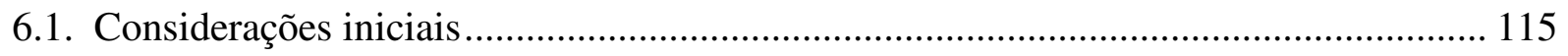

6.2. Cinemática do elemento finito de casca homogêneo ................................................... 116

6.3. Cinemática do elemento finito de casca laminada ....................................................... 122

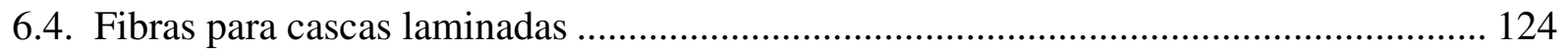

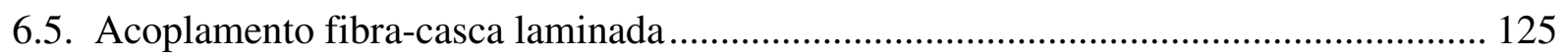

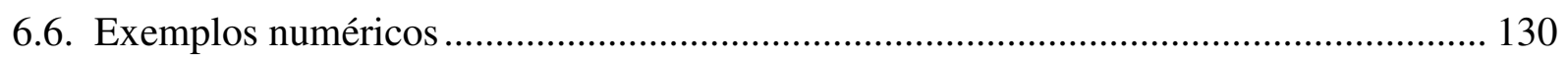

6.6.1. Exemplo 1: Viga engastada reforçada com fibras submetida a grandes deslocamentos....

6.6.2. Exemplo 2: Placa simplesmente apoiada reforçada com fibras ................................. 133

6.6.3. Exemplo 3: Casca cilíndrica laminada reforçada com fibras...................................... 138

6.6.3.1. Casca laminada reforçada com fibras aleatórias ..................................................... 144

6.6.3.2. Casca laminada reforçada com fibras em grandes deslocamentos .......................... 147

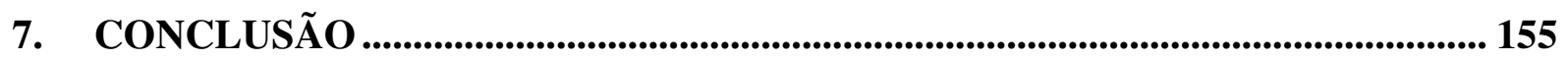

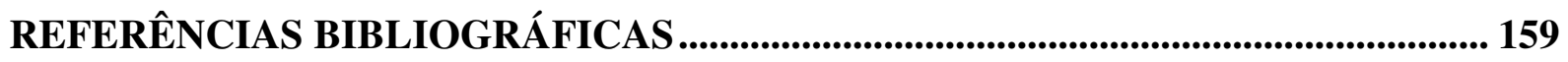

APÊNDICE A - Vetor de forças internas e matriz Hessiana do elemento de fibra simples 167

APÊNDICE B - Resultados para a análise de conformidade do mapeamento fibra-matriz 169 
APÊEDICE C - Gerador de fibras longas e curtas aleatórias

ANEXO A - Polinômios de Lagrange e primeira derivada 183

ANEXO B - Integração numérica em domínios triangulares 185 ANEXO C - Obtenção das coordenadas adimensionais de um ponto contido em um elemento finito triangular 187

ANEXO D - Funções de forma do elemento finito triangular com dez nós 189 



\section{INTRODUÇÃO}

\subsection{CONSIDERAÇÕES INICIAIS}

Compósitos laminados são cada vez mais utilizados nas indústrias em que elevadas relações resistência-peso e rigidez-peso são necessárias ou desejadas (JEYACHANDRABOSE; KIRKHOPE, 1985). Estas relações podem ser obtidas orientandose as fibras em cada lâmina do laminado, em direções predeterminadas, de forma que os parâmetros resistência e rigidez atendam às especificações de projeto (BASAR; ITSKOV; ECKSTEIN, 2000).

Nestas condições, em que se deseja otimizar suas propriedades finais e minimizar o seu peso, é necessário realizar uma análise para prever a resposta não linear geométrica da estrutura sob condições gerais de carregamento (JEYACHANDRABOSE; KIRKHOPE, 1985).

Devido à complexidade dos mecanismos que governam o comportamento de estruturas constituídas por compósitos laminados, e considerando que para a grande maioria das aplicações de interesse prático os modelos analíticos são muito complexos ou mesmo inexistentes, o Método dos Elementos Finitos se tornou uma ferramenta conveniente para a análise de problemas com geometrias arbitrárias e condições gerais de vinculação e carregamento (VENKATESH; RAO, 1985).

Além de estudar o equilíbrio na posição atual da estrutura, outro requisito importante para a concepção de componentes e/ou estruturas constituídas por compósitos laminados é a disponibilidade de modelos e métodos confiáveis para prever a falha do material (BASAR; ITSKOV; ECKSTEIN, 2000), que pode ocorrer por falha na fibra, falha na matriz, pelo descolamento entre a fibra e a matriz e pelo descolamento entre lâminas adjacentes (DANIEL; ISHAI, 2006).

A existência destes mecanismos de falha exige a disponibilidade de formulações e 
métodos que permitam identificar, com precisão, a distribuição das tensões nas fibras, na matriz e das tensões de contato entre as fibras e a matriz, fundamentais para a concepção e análise de falha de estruturas constituídas por este tipo de material (BASAR; ITSKOV; ECKSTEIN, 2000).

Embora o problema da determinação do campo de tensões em sistemas laminados seja tridimensional (HERAKOVICH, 1998; JONES, 1975) o que se observa, de modo geral, é que a grande maioria dos trabalhos disponíveis na literatura apresentam formulações com simplificações cinemáticas, que conduzem à obtenção dos elementos estruturais denominados placas e cascas para a análise do problema de forma aproximada (QATU; SULLIVAN; WANG, 2010).

Além dos desafios inerentes à modelagem matemática e a implementação computacional, a extensão dos elementos finitos de casca para a análise de sistemas laminados reforçados com fibras apresenta como dificuldades adicionais a escolha do modelo mecânico a ser utilizado para descrever o comportamento do meio reforçado e a geração das malhas das superfícies curvas tridimensionais com enrijecedores (LI et al., 1997).

Os métodos existentes para a análise de estruturas laminadas reforçadas com fibras podem ser organizados em pelo menos quatro grandes grupos: os modelos em que o meio laminado heterogêneo é substituído por um homogêneo equivalente; os modelos em que tanto a matriz quanto as fibras são discretizadas com elementos sólidos tridimensionais; os modelos em que a matriz é discretizada com elementos de casca e as fibras com elementos de barra e os modelos em que a rigidez das fibras é distribuída na rigidez do elemento finito de casca (BARUT et al., 2000). Em geral, estes modelos ou dificultam a análise do problema de forma local, ou aumentam o número de graus de liberdade do sistema de equações resultante e/ou exigem a coincidência dos nós das fibras com os nós da matriz na discretização do problema.

A malha de uma superfície curva tridimensional pode ser gerada pela discretização direta da superfície curva ou a partir de um mapeamento de uma malha plana sobre a superfície curva desejada, sendo o segundo caso o mais simples do ponto de vista computacional (LI et al., 1997). Em relação aos enrijecedores, nos modelos convencionais em elementos finitos a malha é disposta de modo que os nós dos elementos finitos de fibra coincidam com os nós dos elementos finitos de casca (LI et al., 1997), restrição que causa bastante dificuldade na geração automática da malha, principalmente em meios reforçados com fibras aleatórias. 


\subsection{DESCRIÇÃO DO PROGRAMA COMPUTACIONAL EXISTENTE}

O Grupo de Mecânica Computacional (GMEC) do Departamento de Engenharia de Estruturas (SET) da Escola de Engenharia de São Carlos da Universidade de São Paulo (EESC/USP) dispõe de um programa computacional para análise não linear física e geométrica de cascas laminadas anisotrópicas em desenvolvimento desde o ano de 2007.

Neste programa, os parâmetros nodais dos elementos finitos de casca utilizados para discretizar o domínio são as posições, diferentemente das formulações clássicas em elementos finitos, que utilizam deslocamentos. As deformações são medidas em relação à configuração inicial do corpo, caracterizando a descrição Lagrangeana Total ou Material do movimento. A medida de deformação utilizada é a de Green associada à Lei Constitutiva de Saint-VenantKirchhoff e ao tensor de tensões de Piola-Kirchhoff de segunda espécie. O equilíbrio é encontrado a partir do Princípio da Mínima Energia Potencial Total e o sistema não linear de equações resultante é resolvido utilizando-se o procedimento iterativo de Newton-Raphson. As ações externas podem ser introduzidas ao sistema de forma total ou incremental.

A partir dos trabalhos de Bonet et al. (2000) e de Coda e Greco (2004), que introduziram uma formulação do método dos elementos finitos com parâmetros nodais em posições para a análise não linear geométrica de estruturas, vários trabalhos, para diferentes aplicações, foram desenvolvidos no SET, demonstrando a eficiência e a aplicabilidade da abordagem, em posições, tanto para a formulação dos problemas, quanto para a posterior implementação computacional.

Exemplos da aplicabilidade e evolução da formulação em posições podem ser observados nos trabalhos desenvolvidos no SET a partir da introdução da formulação por Coda e Greco (2004), como por exemplo, para a análise dinâmica de estruturas formadas por barras (GRECO; CODA, 2006); para a análise não linear geométrica com impacto de domínios elásticos bidimensionais (MARQUES, 2006); para a análise não linear geométrica de estruturas formadas por cascas (CODA; PACCOLA, 2007); para a análise não linear geométrica de impacto bidimensional entre estruturas reticuladas e anteparo rígido curvo (MINSKI, 2008); para a análise não linear geométrica de problemas modelados por pórticos planos e sólidos tridimensionais (MACIEL, 2008); para a análise não linear geométrica de chapas com modelos constitutivos elastoméricos (PASCON, 2008); para a análise não linear 
geométrica de estruturas formadas por cascas considerando variação linear da deformação ao longo da espessura do elemento (CODA; PACCOLA, 2008); para a análise de impacto entre estruturas com comportamento termo-elástico e termo-plástico (CARRAZEDO, 2009); para a análise dinâmica não linear de estruturas formadas por cascas (CODA; PACCOLA, 2009); para a análise não linear geométrica de estruturas laminadas modeladas com elementos tridimensionais de barra considerando o efeito do empenamento da seção transversal (CODA; PACCOLA, 2010); para a análise não linear geométrica de estruturas com interação fluidocasca (SANCHES, 2011); para a análise dinâmica não linear de estruturas formadas por elementos de barra tridimensionais considerando o efeito do empenamento da seção transversal (CODA; PACCOLA, 2011); para a análise de estruturas de aço formadas a frio em situação de incêndio (RIGOBELLO, 2011); para a análise de materiais elásticos e elastoplásticos com gradação funcional em regime de grandes deslocamentos e deformações (PASCON, 2012); para a análise não linear geométrica de cascas laminadas anisotrópicas (CODA; PACCOLA; SAMPAIO, 2013) e; para a análise estática e dinâmica não linear de risers (SANCHEZ, 2013).

Em particular, Coda, Paccola e Sampaio (2013) utilizam a cinemática de ReissnerMindlin para formular um elemento finito de casca laminada anisotrópica. Nesta formulação, laminados reforçados com fibras podem ser analisados por meio de modelos homogêneos equivalentes, que fornecem soluções globais satisfatórias, mas dificultam a identificação das tensões de contato entre as fibras e a matriz, que são necessárias, por exemplo, caso se deseje realizar a análise de falha do laminado.

\subsection{OBJETIVOS}

Com base no exposto nos itens 1.1 e 1.2, definem-se o objetivo geral desta tese no item 1.3.1 e os objetivos específicos e a metodologia no item 1.3.2.

\subsubsection{OBJETIVO GERAL}

O objetivo geral desta tese consiste em desenvolver uma formulação para a inclusão 
de fibras longas e curtas aleatórias nas diversas lâminas de cascas laminadas anisotrópicas com não linearidade geométrica utilizando o método dos elementos finitos com parâmetros nodais em posições sem aumentar o número de graus de liberdade do sistema de equações resultante e sem a necessidade de coincidência dos nós das fibras com os nós da matriz na discretização do problema.

\subsubsection{OBJETIVOS ESPECÍFICOS E METODOLOGIA}

Os objetivos específicos desta tese são:

1.3.2.1. Formular um elemento finito de chapa de ordem qualquer para a análise não linear geométrica de domínios elásticos bidimensionais. Desenvolver e implementar um código computacional baseado na formulação desenvolvida;

1.3.2.2. Formular um elemento finito de barra simples para simular trechos de fibras longas e fibras curtas aleatórias inseridas via gerador automático em domínios bidimensionais. Acoplar a formulação ao código computacional desenvolvido no item 1.3.2.1;

1.3.2.3. Propor estratégia cinemática de elemento unidimensional curvo de ordem qualquer para simular as fibras, possibilitando a perfeita aderência com o domínio bidimensional. Desenvolver e implementar código computacional baseado na cinemática proposta e comparar os resultados obtidos com aqueles obtidos com a cinemática das fibras desenvolvida no item 1.3.2.2;

1.3.2.4. Adaptar as formulações desenvolvidas nos itens 1.3.2.2 e 1.3.2.3 ao elemento de casca laminada e implementar as sub-rotinas necessárias no programa disponível. Simular exemplos e analisar os resultados obtidos;

1.3.2.5. Propor estratégias para a determinação das tensões de aderência e normal de contato nas fibras curvas de ordem qualquer desenvolvidas neste trabalho;

1.3.2.6. Desenvolver um gerador automático de fibras curtas aleatórias e longas para a análise de domínios elásticos bidimensionais e laminados que não aumente o número de graus de liberdade do sistema de equações resultante e que seja independente da coincidência dos nós da matriz. 
Desta forma, o programa computacional de chapa para a análise de domínios bidimensionais reforçados com fibras, retas ou curvas, itens 1.3.2.1-1.3.2.3, e o gerador automático de fibras, tanto para chapa quanto para casca laminada, item 1.3.2.6, foram integralmente desenvolvidos e implementados neste trabalho. Para validar as formulações desenvolvidas e o código computacional implementado foram testados exemplos cuja solução analítica é conhecida, problemas com soluções disponíveis na literatura e uma ferramenta computacional, já validada, que utiliza cinemática de Timoshenko-Reissner denominada barra geral 3D (CODA, 2009; CODA; PACCOLA, 2010; CODA; PACCOLA, 2011).

Para a análise de cascas laminadas reforçadas com fibras, os equacionamentos relacionados à formulação das fibras foram integralmente desenvolvidos e implementados neste trabalho, item 1.3.2.4. As sub-rotinas necessárias para a consideração das fibras resultantes da formulação proposta foram implementadas no programa computacional existente descrito na seção 1.2. Para validar a formulação desenvolvida foram utilizadas ferramentas computacionais disponíveis no grupo de pesquisa, que por sua vez foram validadas com benchmarks disponíveis na literatura.

Para visualizar os resultados obtidos, os códigos desenvolvidos foram acoplados a um software de acesso livre para pós-processamento em elementos finitos 2D e 3D ACADVIEW.

\subsection{CONTRIBUIÇÃO DA TESE}

Embora todos os objetivos especificados no item 1.3 tenham sido desenvolvidos considerando-se as particularidades do programa base deste trabalho, implementado em posições, destaca-se como principal contribuição desta tese o desenvolvimento de uma formulação para a introdução de fibras curvas, longas ou curtas aleatórias, nas diversas camadas que compõem um laminado, modelado aqui com elementos finitos de casca, sem aumentar o número de graus de liberdade do sistema de equações resultante e sem a necessidade de coincidência dos nós das fibras com os nós da matriz na discretização do problema. Conforme proposta, esta formulação permite a simulação de uma grande variedade de compósitos reforçados com fibras, como, por exemplo, aqueles mostrados na Figura 1. 


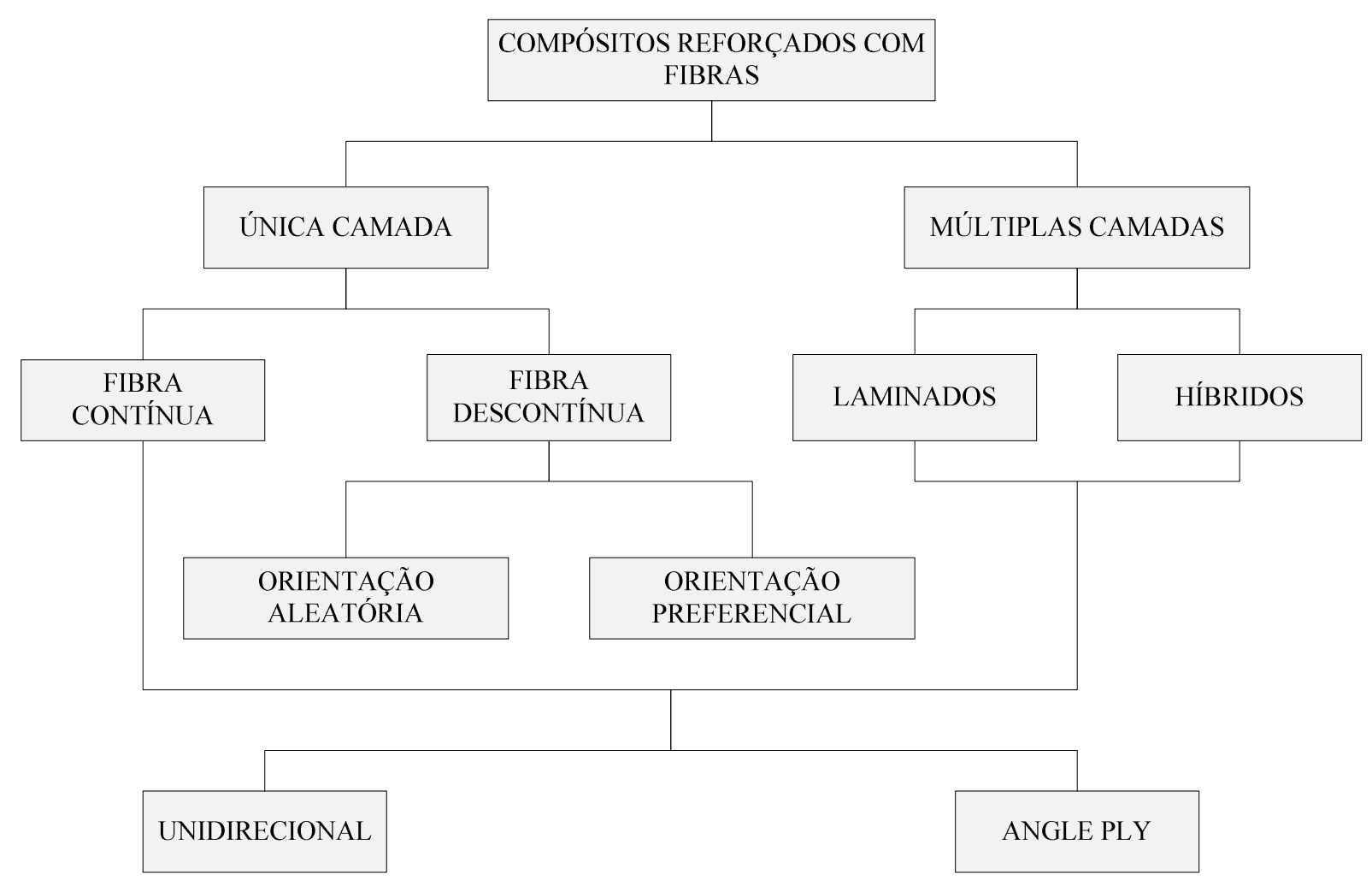

Figura 1 - Classificação dos materiais compósitos reforçados com fibras.

Adaptado de (VISON; SIERAKOWSKI, 1987).

\subsection{ORGANIZAÇÃO DA TESE}

Para apresentar a formulação desenvolvida, além do capítulo de introdução, esta tese está organizada em sete capítulos conforme descrito a seguir.

No Capítulo 2 apresenta-se uma breve revisão bibliográfica desde o surgimento dos primeiros elementos finitos de casca na década de 60 até a sua aplicação para a modelagem de sistemas laminados estruturais nos dias atuais. Esta revisão foi escrita não com o objetivo de esgotar as formulações de cascas disponíveis, pois muitos são os estudos realizados desde o surgimento dos primeiros elementos, mas sim com o objetivo de localizar a formulação desenvolvida neste trabalho dentro do universo de uma grande variedade de formulações disponíveis, evidenciando assim, as suas particularidades e demonstrando que mesmo após quase 50 anos de extensiva pesquisa nestes elementos, o interesse pelo comportamento dos mesmos continua nos dias atuais.

No Capítulo 3 descreve-se o problema de equilíbrio não linear geométrico conforme 
utilizado na resolução dos problemas de interesse deste trabalho e o método adotado para resolver o sistema de equações não lineares resultante da sua aplicação.

No Capítulo 4 descrevem-se a formulação do elemento finito bidimensional de ordem qualquer utilizado para a análise de domínios elásticos reforçados com fibras com não linearidade geométrica; a formulação do elemento de fibra simples; a cinemática do elemento finito curvo de ordem qualquer; o acoplamento fibra-matriz utilizado para introduzir a contribuição das fibras sem aumentar o número de graus de liberdade do sistema de equações resultante e os exemplos numéricos utilizados para validar as formulações desenvolvidas.

No Capítulo 5 descrevem-se as duas estratégias adotadas para a determinação das tensões de contato ao longo das fibras curvas de ordem qualquer introduzidas neste trabalho e os exemplos numéricos utilizados para validar as estratégias utilizadas.

No Capítulo 6 apresentam-se o elemento finito de casca homogêneo; a sua generalização para o caso laminado; a cinemática proposta para a inclusão das fibras curvas em qualquer camada do elemento de casca laminada sem aumentar o número de graus de liberdade do sistema de equações resultantes e os exemplos numéricos que validam o acoplamento fibra-casca proposto.

No Capítulo 7 tem-se a conclusão do trabalho e algumas sugestões para trabalhos futuros.

Por fim, listam-se, em ordem alfabética, as referências bibliográficas consultadas para o desenvolvimento deste trabalho. Salienta-se que todas as referências listadas apresentam contribuições para o entendimento do problema aqui estudado e podem ser consultadas quando detalhes específicos forem necessários. 


\section{REVISÃO BIBLIOGRÁFICA}

\subsection{CONSIDERAÇÕES INICIAIS}

Seja com o objetivo de melhorar continuamente o seu desempenho, seja para melhor compreender seus modos de falha, os materiais compósitos laminados são assunto de grande interesse da engenharia moderna (QATU; SULLIVAN; WANG, 2010), pois, uma vez que é possível combinar as propriedades de seus constituintes em certas direções preferencias e otimizar seu desempenho para atender a especificações de projeto (BASAR; ITSKOV; ECKSTEIN, 2000), o emprego destes materiais na obtenção de estruturas cada vez mais leves e resistentes e, consequentemente, mais delgadas e deslocáveis se intensificou consideravelmente nas últimas décadas (YASIN; KAPURIA, 2013).

Para descrever o comportamento de estruturas altamente deslocáveis constituídas por materiais compósitos laminados deve-se estudar a condição de equilíbrio na configuração atual do corpo, considerando os deslocamentos e deformações ocorridas durante o processo de mudança de configuração independentemente de serem grandes ou pequenos (BARUT et al., 2000) e dispor de modelos e métodos confiáveis para descrever o comportamento e prever a falha do material (BASAR; ITSKOV; ECKSTEIN, 2000).

O estudo da condição de equilíbrio considerando os deslocamentos ocorridos durante o processo de mudança de configuração é conhecido como análise não linear geométrica e seus principais objetivos são descrever a trajetória de equilíbrio da estrutura, ou seja, representar as configurações equilibradas correspondentes aos sucessivos níveis de força aplicada, identificar pontos críticos em que ocorre mudança da condição de equilíbrio de estável para instável e analisar a condição de estabilidade do equilíbrio associada a cada configuração (CRISFIELD, 1991).

Devido às características que a solução de um problema não linear geométrico pode apresentar (Figura 2), um método completo para a resolução de tais problemas deve se auto- 
adaptar às mudanças de direção do carregamento nos pontos limites, manter a estabilidade numérica para as iterações em todas as regiões, incluindo aquelas próximas a pontos críticos e deve ajustar automaticamente o tamanho dos passos de carga para refletir os comportamentos de softening e stiffening da estrutura (YANG; SHIEH, 1990).

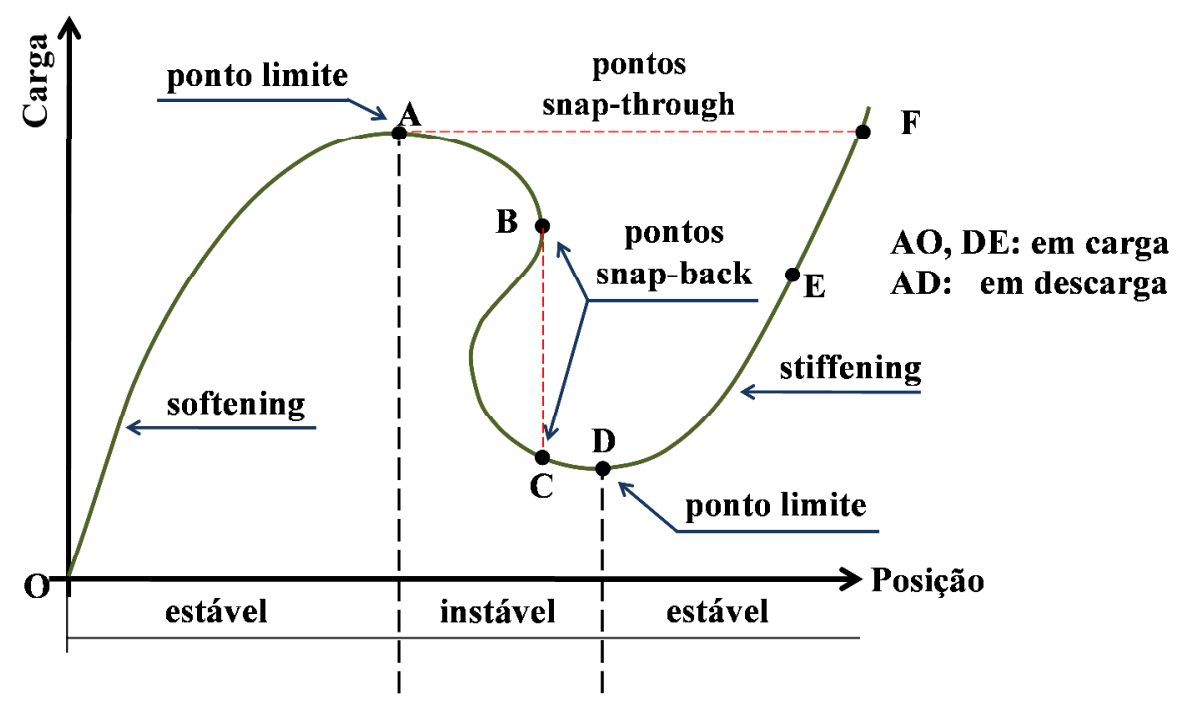

Figura 2 - Características da solução de um problema não linear. Fonte: (YANG; SHIEH, 1990).

Dentre os métodos disponíveis para a resolução de problemas não lineares geométricos citam-se o Método de Newton-Raphson (LUENBERG, 1989), o Método do Controle de Deslocamento (SAMARTIN, 1993), o Método do Comprimento de Arco (CRISFIELD, 1991), o Método do Controle do Trabalho (SAMARTIN, 1993) e o Método do Controle por Deslocamentos Generalizados, que segundo Yang e Shieh (1990) é capaz de representar todas as características apresentadas na Figura 2.

Embora existam métodos mais gerais, adota-se, neste trabalho, o método de NewtonRaphson contemplando também controle de deslocamento, que assegura, no mínimo, ordem dois de convergência (LUENBERG; 1989), e é capaz de capturar o comportamento dos problemas de interesse deste trabalho além de ser mais simples de implementar computacionalmente. Além disto, tem mostrado bom desempenho e conduzido a bons resultados nos problemas até então simulados no GMEC/SET/EESC/USP descritos na Seção 1.2 .

No que se refere ao comportamento e à falha do material, de modo geral, e, desconsiderando o tipo de falha ou mesmo as suas causas, os critérios utilizados definem um limite que deve ser satisfeito pelos materiais quando submetidos a um determinado estado de tensão, que torna imprescindível a disponibilidade de modelos e métodos eficientes para a 
determinação deste campo (BASAR; ITSKOV; ECKSTEIN, 2000).

Embora o problema da determinação do campo de tensão em sistemas laminados seja tridimensional (HERAKOVICH, 1998; JONES, 1975) o que se observa, de modo geral, é que a grande maioria dos trabalhos disponíveis na literatura apresentam formulações com simplificações cinemáticas, que conduzem à obtenção dos elementos estruturais denominados placas e cascas para a análise do problema de forma aproximada (QATU; SULLIVAN; WANG, 2010). Isso ocorre, pois as formulações tridimensionais demandam maior espaço de armazenamento e apresentam maior custo computacional devido ao grande número de variáveis envolvidas no problema.

Embora o surgimento destes elementos date do início da década de 60 e muitos sejam os trabalhos desenvolvidos desde então, o que se observa, é que ainda há grande interesse no desenvolvimento dos elementos finitos de casca não só pela abrangência da sua aplicação na modelagem de diversas estruturas (QATU; SULLIVAN; WANG, 2010) como também pelos desafios inerentes à modelagem matemática e a sua implementação computacional ainda existentes nos dias atuais (YANG et al., 2000).

Desta forma, apresenta-se, a seguir, uma breve revisão bibliográfica desde o surgimento dos primeiros elementos finitos de casca na década de 60 até a sua aplicação para a modelagem de sistemas laminados estruturais nos dias atuais.

\subsection{DOS PRIMEIROS ELEMENTOS DE CASCA ATÉ A SUA APLICAÇÃO A LAMINADOS NOS DIAS ATUAIS}

As equações da elasticidade tridimensional para corpos delimitados por duas superfícies curvas, cuja dimensão normal à superfície média, a espessura, é pequena quando comparada com as demais, não são simples e quase todas as teorias de cascas disponíveis na literatura reduzem o problema tridimensional a um problema bidimensional pela imposição de hipóteses simplificadoras relacionadas a aplicações específicas (QATU; SULLIVAN; WANG, 2010).

Devido ao complexo comportamento apresentado pelas cascas bem como às dificuldades envolvidas na modelagem matemática e numérica deste tipo de estrutura (YANG et al., 2000), muitos pesquisadores têm se dedicado ao seu estudo e diversos trabalhos relacionados ao tema podem ser encontrados na literatura. 
De acordo com Arciniega e Reddy (2005) os elementos finitos utilizados para modelar estruturas do tipo casca podem ser agrupados em quatro tipos: elementos planos, elementos sólidos tridimensionais, elementos degenerados e elementos bidimensionais baseados em uma teoria de casca, sendo os dois últimos os mais comumente utilizados.

Um dos primeiros trabalhos a utilizar o chamado elemento plano, obtido pela superposição de elemento de membrana com elemento de flexão, para a análise de casca foi apresentado por Greene, Strome e Weikel (1961). Estes elementos são de fácil formulação e descrição da geometria na discretização, são fáceis de associar a outros elementos, modelam movimento de corpo rígido livre de deformações e permitem a consideração de condições de carregamento e contorno complexas e, como desvantagens, o desacoplamento entre comportamentos de membrana e flexão, restrição à forma triangular para o tratamento de cascas mais gerais, dificuldade de tratamento das descontinuidades na junção entre elementos e influência da aproximação geométrica, plana, na solução de problemas com superfícies mais gerais (YANG; SAIGAL; LIAW, 1990). A Figura 3, extraída de Yang, Saigal e Liaw (1990), mostra alguns elementos finitos planos relacionados na revisão bibliográfica realizada por estes autores.

\begin{tabular}{|c|c|c|c|c|c|}
\hline Forma & Autores & Ano & $\begin{array}{l}\text { Funções para os } \\
\text { Deslocamentos }\end{array}$ & $\begin{array}{l}\text { Graus de } \\
\text { Liberdade }\end{array}$ & $\begin{array}{c}\text { Número total } \\
\text { de graus de liberdade }\end{array}$ \\
\hline & Zienkiewicz e Cheung [5] & 1965 & $\begin{array}{l}u, v \text { linear, } \\
\text { w cúbico }\end{array}$ & $\begin{array}{l}u, v \\
w, w_{x}, w_{y}\end{array}$ & 20 \\
\hline & Zienkiewicz et al. [6] & 1968 & $\begin{array}{l}u, v \text { linear, } \\
\text { w cúbico }\end{array}$ & $\begin{array}{l}u, v \\
w, w_{x}, w_{y}\end{array}$ & 10 \\
\hline & Clough e Johnson [7] & 1968 & $\begin{array}{l}\text { u, v linear, } \\
\text { w cúbico }(\mathrm{HCT})\end{array}$ & $\begin{array}{l}u, v \\
w, w_{x}, w_{y}\end{array}$ & 15 \\
\hline & Dawe [8] & 1972 & $\begin{array}{l}u, v \text { linear, } \\
\text { w quadrático }\end{array}$ & $\begin{array}{l}\text { vértices: } \mathrm{u}, \mathrm{v}, \mathrm{w} \\
\text { nó central: } \mathrm{w}_{\mathrm{n}}\end{array}$ & 12 \\
\hline & Argyris et al. [9] & 1977 & $\begin{array}{l}u, v \text { linear } \\
w \text { cúbico }\end{array}$ & $\begin{array}{l}u, v, w \\
w_{x}, w_{y}, w_{x y}\end{array}$ & 18 \\
\hline & Meek e Tan [10] & 1986 & $\begin{array}{l}u, v, w \\
\text { quadrático }\end{array}$ & $\begin{array}{l}u, v, w \text {; } \\
\text { Seis Loof nodes (1/3 e } \\
2 / 3 \text { de cada lado): } w_{n}\end{array}$ & 24 \\
\hline
\end{tabular}

Figura 3 - Alguns elementos planos para a análise de cascas. Fonte: (YANG; SAIGAL; LIAW, 1990). 
O primeiro elemento de casca degenerado é atribuído a Ahmad, Irons e Zienkiewicz (1970). Os elementos degenerados são obtidos pela discretização direta das equações tridimensionais da mecânica do contínuo e esta discretização é então degenerada pela introdução de hipóteses relacionas às normais à superfície média do elemento (YANG; SAIGAL; LIAW, 1990). Embora as hipóteses fundamentais por trás da obtenção do elemento de casca degenerado e do elemento bidimensional pela teoria clássica de cascas sejam as mesmas, a redução à forma final é obtida numericamente no primeiro caso e analiticamente no último. Consequentemente, a abordagem que utiliza a teoria clássica é mais complexa matematicamente (YANG; SAIGAL; LIAW, 1990). A Figura 4, extraída de Gilewski e Radwaflska (1991), mostra a diferença na obtenção dos elementos degenerados e bidimensionais.

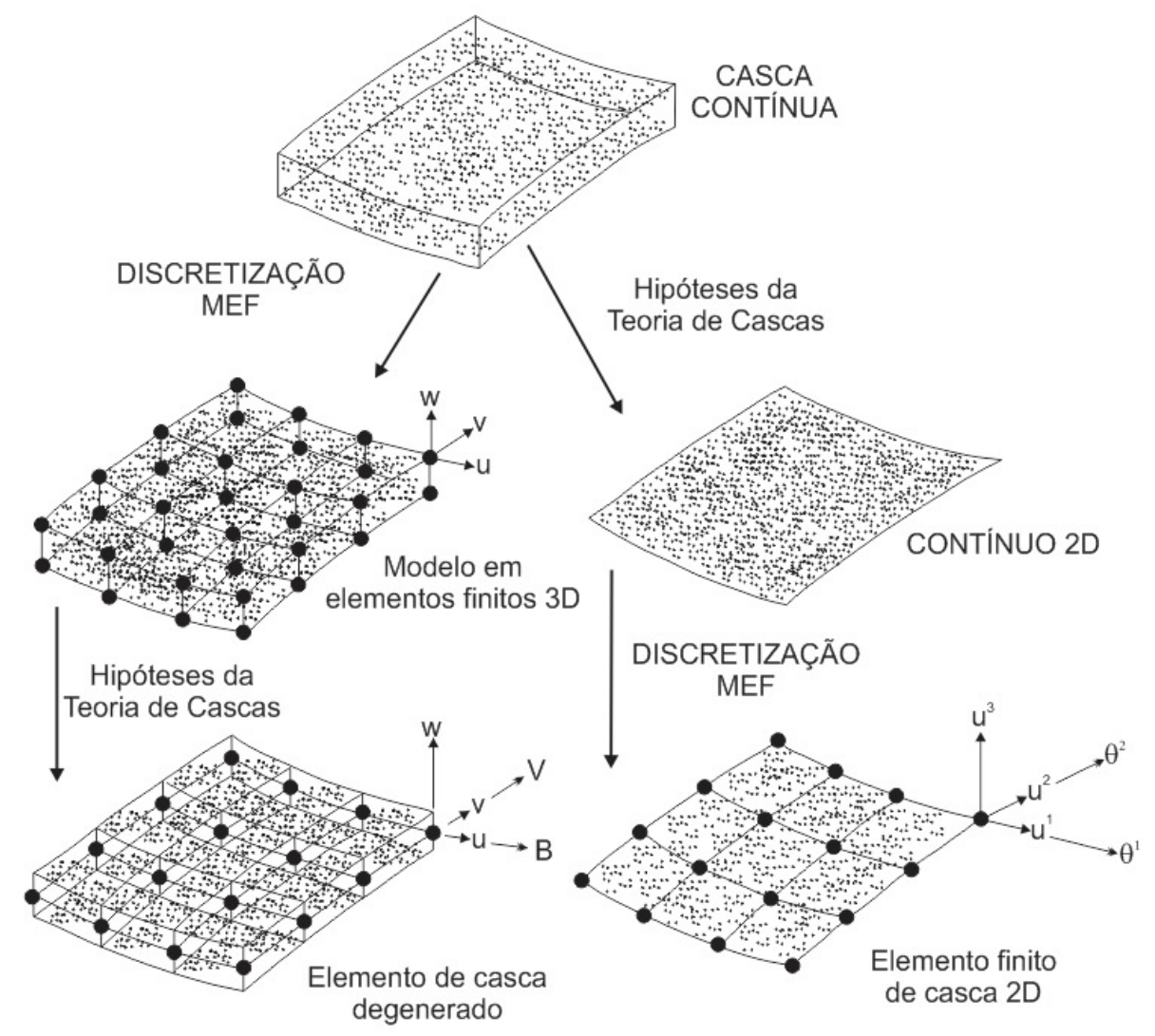

Figura 4 - Comparação de dois conceitos básicos utilizados na obtenção de elementos de casca. (a) elementos degenerados e (b) elementos bidimensionais. Fonte: (GILEWSKI; RADWAFLSKA, 1991).

Os elementos degenerados se comportam de forma satisfatória para a análise de placas e cascas grossas, mas apresentam travamento por cisalhamento quando a placa ou casca se torna muito fina. Uma forma de evitar o travamento nestes elementos é a utilização de 
esquemas de integração reduzida (YANG; SAIGAL; LIAW, 1990).

Os elementos tridimensionais surgem como uma proposta de ferramenta geral para a resolução de problemas arbitrários da mecânica dos sólidos (YANG et al., 2000). Yang et al. (2000) enumeram as características que estes elementos devem apresentar objetivando-se generalidade: (i) não travar para materiais incompressíveis; (ii) bom comportamento na flexão; (iii) não travar na análise de problemas que exigem o emprego de elementos muito finos; (iv) devem ser insensíveis à distorção; (v) boa precisão com utilização de malhas grosseiras; (vi) simples de implementar para modelos constitutivos não lineares e (vii) apresentar bons resultados. A terceira característica é a que merece maior destaque no presente caso, pois permite a simulação de problemas de cascas com elementos sólidos tridimensionais, que são relativamente mais simples de formular que os outros elementos (YANG et al., 2000). A utilização de elementos sólidos tridimensionais evita a necessidade de introdução de rotações finitas como variáveis no problema, necessárias em algumas formulações para a análise de cascas finas e permite a aplicação direta de equações constitutivas tridimensionais (YANG et al., 2000). Por outro lado, estes elementos conduzem a discretizações com muitos graus de liberdade e necessitam que todas as características citadas sejam consideradas na sua obtenção (YANG et al., 2000).

Mais recentemente, com o advento da computação paralela, a utilização de elementos de alta ordem para a análise de sólidos e cascas com comportamento elástico isocórico ou não vem sendo abordada como mostram os trabalhos de Pascon (2008) e Pascon (2012) e outros citados por ele.

Existe uma grande quantidade de trabalhos disponíveis na literatura para cada um dos tipos de elemento mencionados, abordando os mais diversos aspectos, seja geometria, material, tipo de análise, etc. Como não é objetivo deste trabalho evidenciar as peculiaridades inerentes a cada uma destas abordagens, apenas evidenciar a diferença destas para a que é aqui utilizada, relacionam-se a seguir alguns artigos de revisão bibliográfica sobre o tema que contemplam uma grande variedade de aplicações e evidenciam a quantidade de trabalhos desenvolvidos.

Yang, Saigal e Liaw (1990) apresentam uma revisão bibliográfica com 287 artigos publicados no período de 1965 a 1986 contendo os avanços obtidos na formulação de elementos finitos para análise de cascas finas neste período. Os autores apresentam uma série de trabalhos desenvolvidos com elementos planos, axissimétricos, curvos, elementos de casca do tipo DKT (Discrete Kirchhoff Theory), elementos degenerados, etc., e a extensão destes para análises estáticas e dinâmicas, linear e não linear, compósitos laminados, etc. 
Outro trabalho de revisão bibliográfica que contempla quase o mesmo período, 1965 a 1988, foi apresentado por Gilewski e Radwaflska (1991). Estes autores relacionam 350 artigos destinados à análise de cascas moderadamente espessas. Abordam elementos bidimensionais, degenerados, modelos híbridos, mistos e em tensão. Apresentam ainda uma série de artigos destinados à análise de instabilidade, dinâmica, não linear geométrica, não linear física, de cascas laminadas e sanduíche, transferência de calor e de revisão bibliográfica.

Mackerle (1993) apresenta uma relação com 248 artigos em elementos finitos e 21 artigos em elementos de contorno publicados no período de 1990 a 1992 destinados à análise de cascas. Os trabalhos relacionados abordam problemas bi e tridimensionais, lineares e não lineares, estáticos e dinâmicos, problemas de instabilidade, etc.

Yang et al. (2000) apresentam uma relação com 379 artigos publicados no período de 1985 a 2000. Os autores fazem uma revisão bibliográfica agrupando trabalhos desenvolvidos com elementos degenerados, integração reduzida com estabilização, aproximação por modos incompatíveis, aproximações baseadas em enriquecimento de deformações, elementos tridimensionais, elementos com drilling, formulações co-rotacionais e elementos para a análise de laminados.

Mackerle (2002) apresenta uma relação com 398 referências bibliográficas entre artigos, dissertações, teses, revisões e livros, em elementos finitos e elementos de contorno, publicados no período de 1999 a 2001 para a análise de cascas. Os trabalhos relacionados abordam problemas bi e tridimensionais, análise linear e não linear, estática e dinâmica, análises sísmicas, problemas de impacto, térmicos, instabilidade, fratura, delaminação, contato com e sem atrito, modelagem adaptativa, problemas estocásticos, esquemas de integração, fundações elásticas, modelos constitutivos, etc. $\mathrm{O}$ autor destaca que esta relação não inclui trabalhos de interação fluido-estrutura, problema de otimização, cascas inteligentes e geração de malha.

Para a análise de estruturas laminadas reforçadas com fibras, os métodos existentes, podem ser organizados em pelos menos quatro grandes grupos: os modelos em que o meio laminado heterogêneo é substituído por um homogêneo equivalente; os modelos em que tanto a matriz quando a fibra são discretizadas com elementos sólidos tridimensionais; os modelos em que a matriz é discretizada com elementos de casca e as fibras com elemento de barra e os modelos em que a rigidez da fibra é distribuída na rigidez do elemento finito de casca (BARUT et al., 2000).

A formulação mais simples para a análise de materiais compósitos laminados 
considera o meio como uma única camada equivalente usando a Teoria Clássica de Laminados (YANG et al., 2000). A Teoria Clássica de Laminados é uma extensão direta da Teoria Clássica de Placas, com cinemática de Kirchhoff-Love, para a análise de laminados (DANIEL; ISHAI, 2006). Esta teoria é válida para problemas não suscetíveis aos efeitos relacionados à espessura do material (YANG et al., 2000).

Para superar as limitações da Teoria Clássica de Laminados outras aproximações foram propostas como, por exemplo, a Teoria de Primeira Ordem que considera o efeito das Deformações por Cisalhamento, que utiliza como hipótese a cinemática de Reissner-Mindlin, (DANIEL; ISHAI, 2006).

Tanto a Teoria Clássica de Laminados quanto a Teoria de Primeira Ordem condensam as camadas do laminado em uma única camada equivalente e, portanto, não modelam o empenamento da seção transversal e resultam em tensões incompatíveis entre lâminas adjacentes (YANG et al., 2000). Surgem então as Teorias de Ordem Superior que, basicamente, buscam produzir uma correta variação da solução em deslocamentos e tensões ao longo da espessura de laminados a partir de hipóteses cinemáticas adotadas para descrever o comportamento dos deslocamentos (LO; CHRISTENSEN; WU, 1997; LEVISON, 1980; REDDY, 1983; BERT, 1984; REDDY, 1984; PHAN; REDDY, 1985; REDDY; LIU, 1985; KANT; KOMMINENI, 1992; LIANGXIN; ZHIYU, 1992; KANT; KOMMINENI, 1994; ARCINIEGA; REDDY, 2005; LIU; SOH, 2006; ZHANG; YANG, 2009; RAMESH et al., 2009), etc.

É possível encontrar uma abrangente revisão bibliográfica sobre os elementos de casca para a análise de laminados nos trabalhos de Yang, Saigal e Liaw (1990) e Yang et al. (2000).

Além destes, citam-se ainda, os trabalhos de revisão de Qatu, Sullivan e Wang (2010), que reúne 199 artigos publicados no período de 2000 a 2009 destinados a análise dinâmica de cascas laminadas e de Qatu, Asadi e Wang (2012) que relacionam 315 artigos destinados à análise estática de cascas laminadas publicados no período de 2000 a 2010.

Em particular, relacionam-se a seguir, alguns trabalhos em que a contribuição dos enrijedores para a energia é feita de forma distinta das estratégias que consideram um meio equivalente.

Zudans (1968) e Zudans (1970) desenvolvem um elemento finito plano retangular com quatro nós e cinco graus de liberdade por nó. Nesta formulação, obtida a partir do princípio dos trabalhos virtuais, os enrijecedores podem estar em qualquer posição da linha média do elemento de casca e são distribuídos nos nós dos elementos de casca.

Venkatesh e Rao (1983) apresentam um elemento finito retangular para a análise de 
cascas laminadas reforçadas com fibras. O elemento de casca laminada possui 48 graus de liberdade e as fibras são modeladas com elementos de viga laminada curvos com 16 graus de liberdade. Venkatesh e Rao (1985) empregam o mesmo elemento para a análise de cascas de revolução.

Bhimaraddi, Carr e Moss (1989) apresentam um elemento de casca de revolução com 64 graus de liberdade enrijecido com elemento de viga laminada curvo com 24 graus de liberdade.

Liao e Reddy (1990) utilizam um elemento de casca degenerado associado a um elemento de viga curvo degenerado para simular meios reforçados. O elemento de casca é retangular, a formulação Lagrangeana total e o método de Newton-Raphson é utilizado para descrever a trajetória de equilíbrio.

Li et al. (1997a) apresentam um elemento finito para a análise auto adaptativa de placas e cascas reforçadas com fibras. Os elementos de cascas são quadrilaterais e é necessária a coincidência dos nós da fibra com os nós do elemento de casca na discretização. Posteriormente, (LI et al., 1997b) superam esta limitação utilizando um elemento de viga cuja contribuição é distribuída nos nós do elemento de casca.

Samanta e Mukhopadhyay (1999) apresentam um elemento finito de casca para a análise não linear geométrica de cascas reforçadas com fibras. Este elemento é obtido pela superposição de um elemento de membrana com 12 graus de liberdade e um elemento de placa também com 12 graus de liberdade, mas o elemento resultante possui 18 graus de liberdade devido à imposição de restrições cinemáticas. O problema não linear é formulado com as Equações de von Karman's e resolvido com o método de Newton-Raphson. O elemento de fibra reto pode estar em qualquer posição do elemento de casca.

Barut et al. (2000) apresentam um elemento finito de casca para a análise não linear geométrica de cascas laminadas reforçadas com fibras. Os elementos de casca são triangulares com 5 graus de liberdade por nó e as fibras são modeladas com elementos de viga. Ambos os elementos consideram os efeitos das deformações transversais e anisotropia. A formulação obtida a partir do principio dos trabalhos virtuais é co-rotacional com descrição Lagrangeana total do movimento e as equações finais são escritas apenas em termos dos graus de liberdade do elemento de casca.

Prusty e Satsangi (2001) e Prusty (2003) apresentam um elemento finito de casca com fibra degenerado para a análise estática linear de estruturas oceânicas e navios. O elemento retangular isoparamétrico de casca possui 8 nós e 5 graus de liberdade por nó e as fibras são modeladas com elementos de viga curvos com 3 nós. Nesta formulação, a contribuição da 
fibra é distribuída para os nós do elemento de casca.

Embora muitos elementos de casca tenham sido desenvolvidos nos últimos cinquenta anos, observa-se que os mesmos são muito distintos entre si, seja pela geometria ou pela interpolação das variáveis de interesse, seja pela análise realizada, pelo tipo material empregado na análise, e no caso dos elementos enrijecidos, pela forma de introdução das fibras no elemento. Da mesma forma, a cinemática do elemento finito apresentado neste trabalho é bastante distinta daquelas apresentadas ao longo deste capítulo. O presente elemento de casca, triangular, curvo, isoparamétrico, possui dez nós e sete graus de liberdade por nó totalizando setenta graus de liberdade por elemento. Estes parâmetros nodais, em posições, diferentemente de todas as formulações pesquisadas, que são em deslocamentos, são as três translações, as três componentes de um vetor generalizado e a taxa de variação da deformação ao longo da espessura que evita o fenômeno do travamento volumétrico. Além disto, somente a superfície de referência é discretizada. A generalização deste elemento para análise de cascas laminadas formadas por qualquer número de lâminas não aumenta o número de graus de liberdade do elemento. Isto é possível pela introdução de variáveis simples para a consideração das camadas e é com base neste elemento que uma formulação para a consideração de fibras espalhadas nestas camadas também sem aumentar o número de graus de liberdade do problema é apresentada originalmente neste trabalho. 


\section{O PROBLEMA DE EQUILÍBRIO NÃO LINEAR GEOMÉTRICO}

\subsection{CONSIDERAÇÕES INICIAIS}

O problema central da elasticidade não linear tridimensional consiste em encontrar a posição de equilíbrio de um corpo elástico que após ser submetido a um conjunto de forças aplicadas passa de uma configuração de referência $\Omega^{0}$ a uma configuração deformada $\Omega$, caracterizada por um mapeamento $\varphi$ chamado de função mudança de configuração (CIARLET, 1988).

Para determinar as três componentes de deslocamento, as seis componentes de tensão e as seis componentes de deformação em um ponto de um corpo tridimensional, têm-se três equações de equilíbrio, seis relações deformação-deslocamento e seis relações tensãodeformação (ODEN, 1967; VALLIAPPAN, 1981; VILLAÇA; GARCIA， 2004; SOKOLNIKOFF, 1956).

Considerando que as equações de equilíbrio e as relações deformação-deslocamento são válidas para qualquer material e que dois corpos, de mesmo tamanho e forma, compostos por materiais diferentes, quando submetidos ao mesmo estímulo, geralmente não apresentam a mesma distribuição de forças resultantes, faz-se necessário introduzir informações a estas relações que permitam distinguir as diferentes respostas para diferentes materiais dos quais os corpos possam ser constituídos e assim, caracterizar completamente o seu comportamento. Estas informações relacionadas à resposta do material são introduzidas ao problema por meio das equações constitutivas. Estas equações estabelecem relação entre as tensões internas e as deformações, cuja intensidade depende das propriedades mecânicas do material (ODEN, 1967; VALLIAPPAN, 1981; VILLAÇA; GARCIA, 2004; SOKOLNIKOFF, 1956).

Em particular, é possível integrar as equações diferenciais para um conjunto de condições de contorno bastante simplificadas e obter as soluções procuradas em qualquer 
ponto do corpo. Na prática, no entanto, realizar a integração analiticamente nem sempre é uma tarefa fácil e uma alternativa para esta abordagem conhecida como vetorial pode ser desenvolvida a partir de considerações sobre as quantidades escalares trabalho e energia (TAUCHERT, 1974).

O objetivo deste capítulo é descrever o problema de equilíbrio não linear geométrico, formulado com base em considerações sobre as quantidades escalares trabalho e energia, conforme utilizado na resolução dos problemas de interesse deste trabalho e o método adotado para resolver o sistema de equações não lineares resultante da sua aplicação.

Desta forma, apenas os aspectos gerais da formulação do problema de equilíbrio não linear geométrico pela abordagem posicional são apresentados. As particularidades relacionadas ao tratamento da energia de deformação para a resolução completa do problema são detalhadas no Capítulo 4 para elementos de chapa reforçados com fibras e no Capítulo 6 para elementos de casca laminada reforçados com fibras. Destaca-se ainda que existem outras formas de tratar o problema de equilíbrio não linear geométrico disponíveis na literatura, em geral, bem mais complexas do que a formulação aqui apresentada, ver por exemplo (CIARLET, 1988). A formulação baseada em posições diminui o grau de complexidade das operações relacionadas à regra da cadeia para elementos finitos isoparamétricos e facilita a obtenção da formulação Lagrangeana Total.

\subsection{DESCRIÇÃO DO PROBLEMA DE EQUILÍBRIO}

O comportamento de um corpo em equilíbrio fica completamente determinado conhecendo-se, em qualquer um de seus pontos em uma configuração qualquer, as três componentes de deslocamento, as seis componentes de tensão e as seis componentes de deformação, para uma dada condição de carregamento externo e vinculação no contorno (ODEN, 1967).

Define-se, neste trabalho, a configuração do corpo como a posição ocupada simultaneamente por todas as suas partículas em um dado instante de tempo $t$, desconsiderado neste estudo.

Desta forma, seja $\Omega^{0}$ a configuração de referência natural indeformada de um corpo, $\Omega$ a configuração atual deformada e $F_{j}$ um conjunto de forças externas que atuam sob o corpo (Figura 5). 
Sob a ação das forças externas, os corpos se deformam e se deslocam e os pontos $X \in \Omega^{0}$ passam a ocupar pontos $Y \in \Omega$ caracterizando a mudança de configuração do corpo descrita pela função $\varphi$.

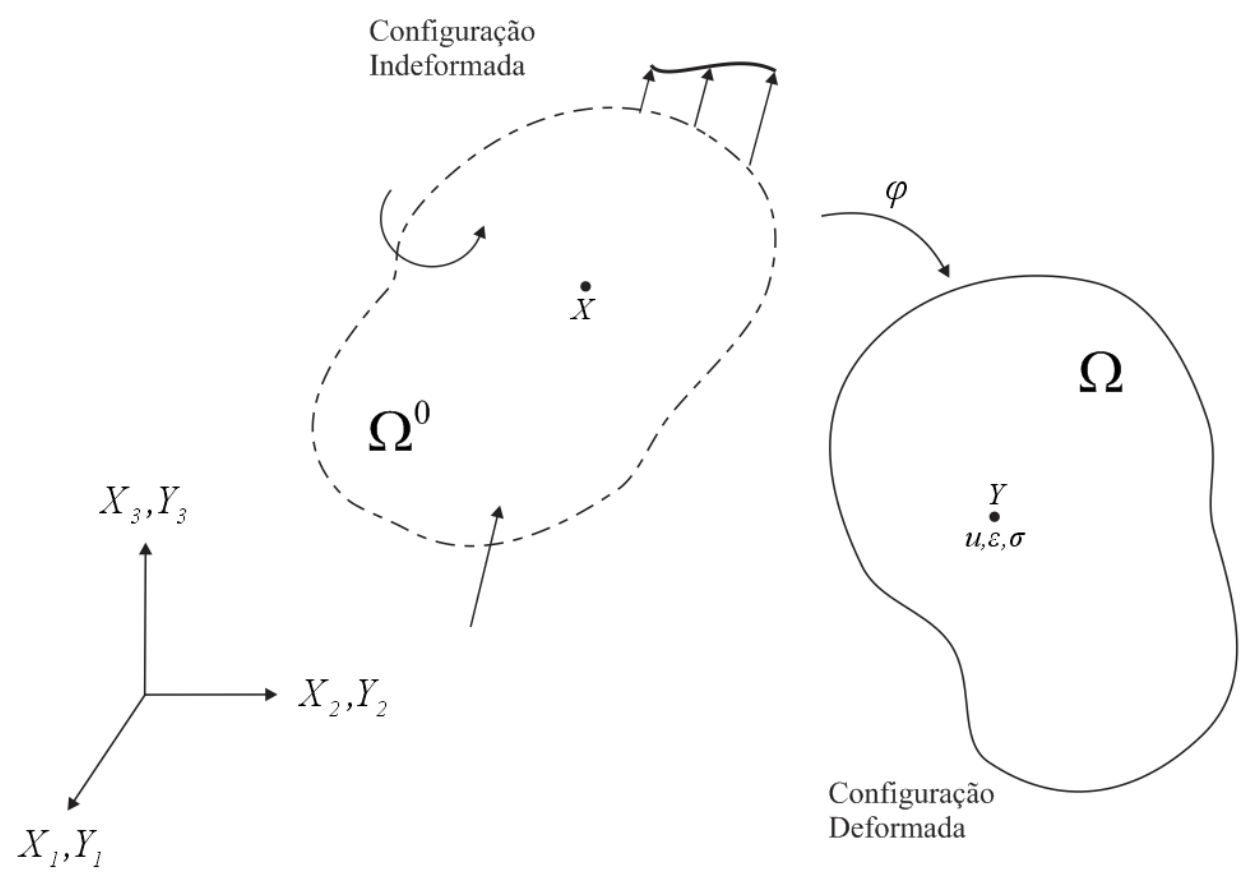

Figura 5 - Configuração de um corpo em equilíbrio.

Durante o processo de mudança de configuração governado pela Primeira Lei da Termodinâmica (TAUCHERT, 1974), a energia total gerada pelo sistema, desprezando-se a contribuição da energia cinética e considerando que o processo é adiabático, é dada por:

$$
\Pi=W+T
$$

sendo $\Pi$ a energia potencial total do sistema, $W$ a energia interna de deformação escrita, neste trabalho, em função das posições e $T$ a energia potencial total das forças externas aplicadas dada por:

$$
T=-F_{j} Y_{j}
$$

onde $F_{j}$ são as forças externas aplicadas e $Y_{j}$ as respectivas posições de aplicação destas forças. 
Considerando-se que as forças são conservativas, ou seja, não dependem das posições onde atuam, aplica-se o Princípio da Mínima Energia Potencial Total (TAUCHERT, 1974) na Eq. (3.1) para resolver a condição de equilíbrio estático do problema não linear geométrico, ou seja,

$$
g_{j}=\frac{\partial \prod}{\partial Y_{j}}=\frac{\partial W}{\partial Y_{j}}-F_{j}=F_{j}^{i n t}-F_{j}=0 .
$$

$\mathrm{Na}$ Eq. (3.3), $F_{j}^{\text {int }}$ é o vetor de forças internas ou vetor gradiente da energia de deformação e as posições atuais dos pontos do corpo, $Y_{j}$, são as incógnitas do problema.

O Princípio da Mínima Energia Potencial Total estabelece que de todas as possíveis posições que satisfazem as restrições do problema, a correta é aquela que faz a energia potencial total do corpo um mínimo (TAUCHERT, 1974), ou seja, ao se assumir uma posição tentativa diferente da posição de equilíbrio, a Eq. (3.3) não é satisfeita. Nesta condição, $g_{j}$ é o vetor desbalanceamento e uma estratégia de resolução de sistemas de equações não lineares deve ser adotada para que se encontre uma solução para o problema.

Neste trabalho, o vetor desbalanceamento, $g_{j}$, é expandido em Série de Taylor em torno de uma solução inicial tentativa definida como $Y_{l}^{0}$, ou seja,

$$
g_{j}\left(Y_{l}\right)=g_{j}\left(Y_{l}^{0}\right)+\left.\frac{\partial g_{j}}{\partial Y_{k}}\right|_{\left(Y_{l}^{0}\right)} \Delta Y_{k}+O_{j}^{2}=0
$$

Desprezando-se os termos de ordem superior, a Eq. (3.4) pode ser reescrita como:

$$
\Delta Y_{k}=-\left(\left.\frac{\partial g_{j}}{\partial Y_{k}}\right|_{\left(Y_{l}^{0}\right)}\right)^{-1} g_{j}\left(Y_{l}^{0}\right) .
$$

$\mathrm{Na}$ Eq. (3.5), $\Delta Y_{k}$ é o incremento ou a correção da solução e

$$
\left.\frac{\partial g_{j}}{\partial Y_{k}}\right|_{\left(Y_{l}^{0}\right)}=\left.\frac{\partial^{2} W}{\partial Y_{k} \partial Y_{j}}\right|_{\left(Y_{l}^{0}\right)}
$$


é a matriz Hessiana ou matriz de rigidez tangente do problema.

Uma vez determinada a correção da solução $\Delta Y_{k}$ na Eq. (3.5) pode-se melhorar a solução tentativa pela expressão:

$$
Y_{l}=Y_{l}^{0}+\Delta Y_{k}
$$

Com esta nova aproximação para a solução do problema repete-se o procedimento até que o incremento $\Delta Y_{k}$ ou o desbalanceamento $g_{j}$ sejam suficientemente pequenos, ou seja, quando certa tolerância em relação à medida, ou norma, for verificada.

A medida adotada neste trabalho para o controle sobre a convergência é definida em função do incremento $\Delta Y_{k}$ e da posição inicial tentativa $Y_{l}^{0}$ pela expressão:

$$
\frac{\sqrt{\Delta Y_{k} \Delta Y_{k}}}{\sqrt{X_{i} X_{i}}} \leq \text { tol } \text {. }
$$

Adota-se como primeira posição tentativa, $Y_{l}^{0}=X_{i}$, a configuração de referência do corpo.

Uma vez satisfeito o critério dado na Eq. (3.8), a solução $Y_{l}$ na Eq. (3.7) é aceita como a configuração de equilíbrio do corpo. Caso contrário, o procedimento é repetido até que a Eq. (3.8) seja satisfeita.

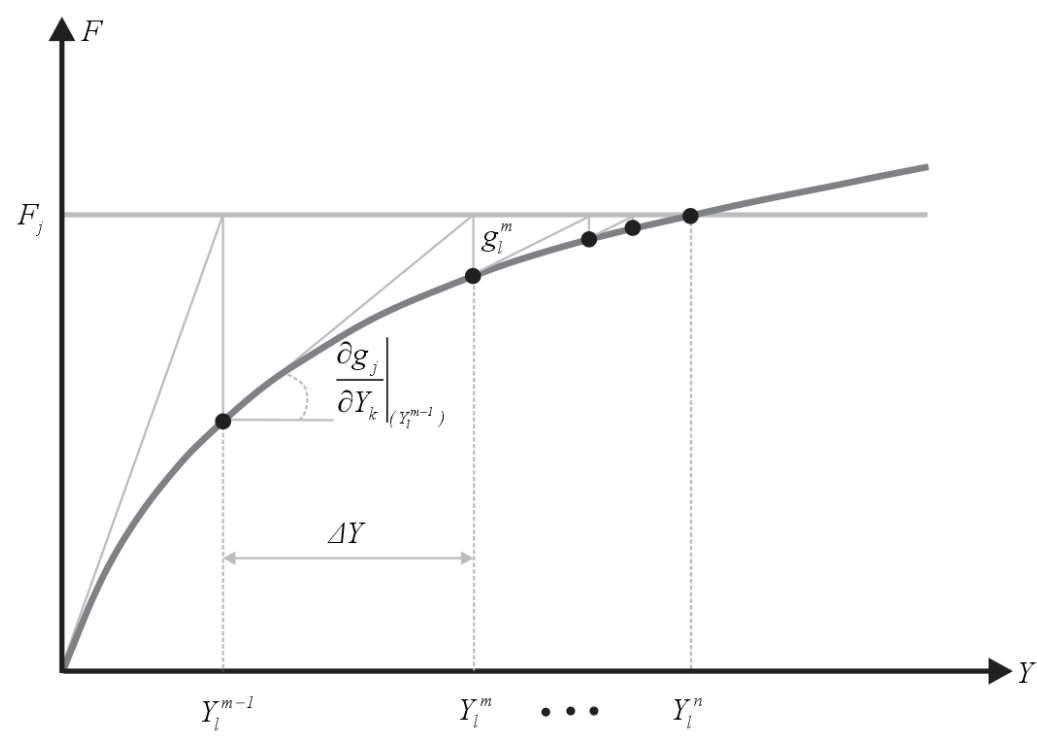

Figura 6 - Representação gráfica do Método de Newton-Raphson. 


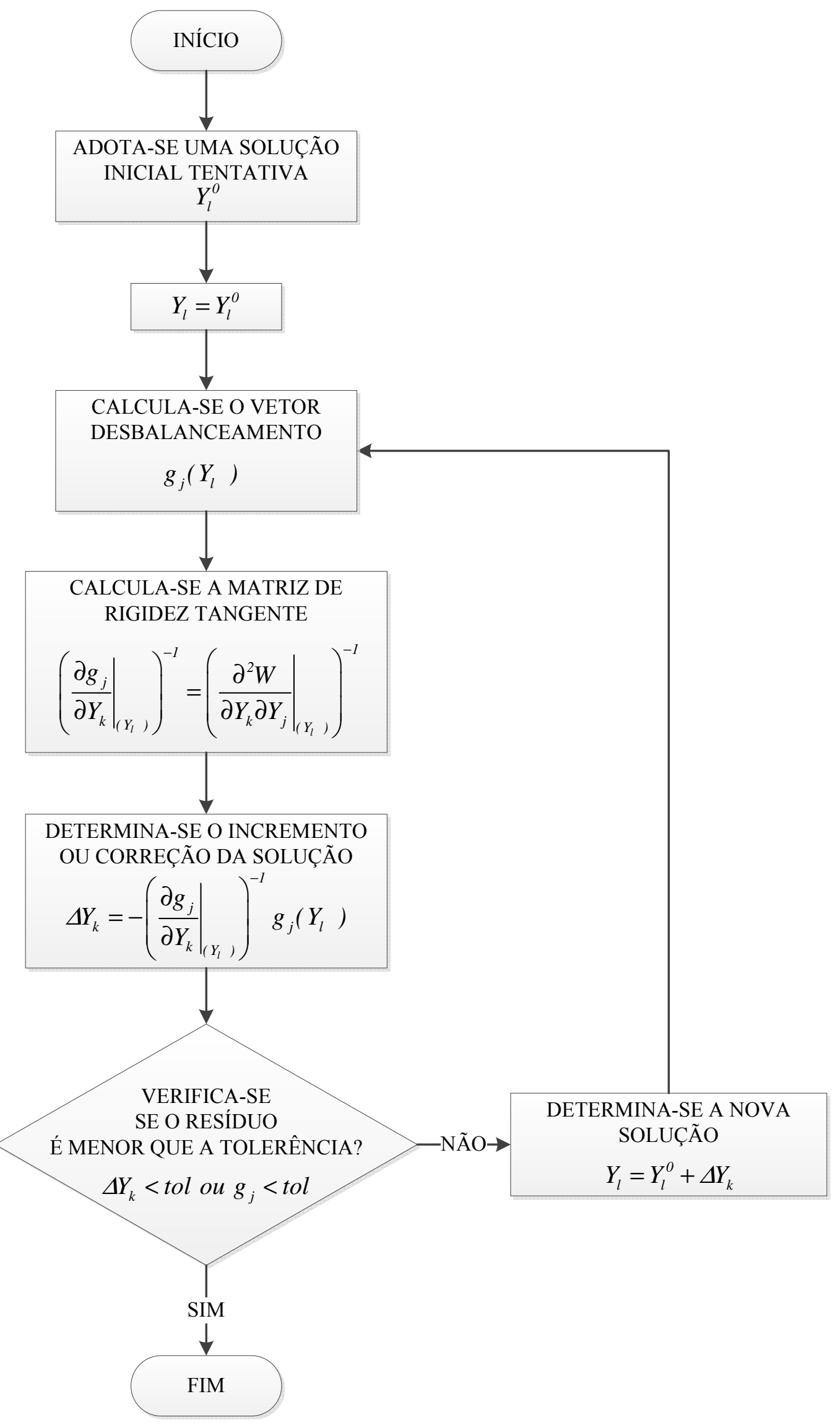

Figura 7 - Fluxograma com o algoritmo para implementação computacional do Método de Newton-Raphson. 
A representação gráfica deste procedimento conhecido como Método de NewtonRaphson com a identificação das grandezas envolvidas pode ser visualizada na Figura 6 e um fluxograma básico para a sua implementação computacional é mostrado na Figura 7.

Destaca-se que as ações externas que dão início ao processo iterativo podem ser introduzidas ao sistema de forma total ou incremental.

Este procedimento, da forma como apresentado, pode ser aplicado na resolução de muitos problemas de interesse da mecânica dos sólidos. Neste trabalho, este problema de equilíbrio é particularizado para a resolução de estruturas laminadas reforçadas com fibras utilizando-se elementos finitos de casca laminada para discretizar a matriz e elementos finitos unidimensionais de ordem qualquer para discretizar as fibras.

Desta forma, $W$ na Eq. (3.6) é dada pelas contribuições da energia da casca e da fibra e a obtenção do termo $\frac{\partial^{2} W}{\partial Y_{k} \partial Y_{j}}$ que permite a resolução do problema é apresentada nos próximos capítulos.

A energia $W$ utilizada neste trabalho, tanto para a casca quanto para as fibras, é a que descreve um Material Saint-Venant-Kirchhoff (CIARLET, 1988), ou seja, a sua Lei Constitutiva é dada pela relação linear entre o tensor de tensões de Piolla-Kirchhoff de segunda espécie e o tensor de deformações de Green-Lagrange (CIARLET, 1988; HOLZAPFEL, 2004; OGDEN, 1984).

Em geral, a Lei Constitutiva de Saint-Venant-Kirchhoff é adequada para a análise de problemas que apresentam grandes deslocamentos e pequenas deformações. Neste caso, o material se comporta semelhantemente àqueles que seguem a Lei de Hooke elástico-linear (CRISFIELD, 1991). Quando as deformações aumentam, a Lei de Saint-Venant-Kirchhoff se afasta da Lei de Hooke e não impede a degeneração do material, ou seja, a sua utilização para problemas com grandes deformações fica prejudicada.

Nas aplicações de interesse da engenharia, tais como as que empregam materiais metálicos e cerâmicos, o uso da Lei de Saint-Venant-Kirchhoff é adequado, pois os níveis de deformação admitidos por estes materiais são pequenos. 


\section{DOMÍNIOS BIDIMENSIONAIS REFORÇADOS COM FIBRAS}

\subsection{CONSIDERAÇÕES INICIAIS}

Matrizes reforçadas com fibras constituem uma classe de materiais compósitos amplamente utilizados na fabricação de equipamentos esportivos, estruturas aeroespaciais, automotivas, civis, offshore, e em muitas outras aplicações (HERAKOVICH, 1998).

O exemplo mais comum de compósito reforçado com fibras na construção civil é o concreto armado que combina o baixo custo do concreto e a sua resistência à compressão com a ductilidade e a resistência à tração do aço.

Muitos trabalhos experimentais e teóricos destinados à abordagem de materiais reforçados com fibras podem ser encontrados na literatura. Em geral estes trabalhos buscam entender melhor o comportamento deste tipo de material e/ou resolver problemas de engenharia relacionados.

Diferentes estratégias são adotadas para incorporar fibras em uma matriz. Nos trabalhos de Radtke, Simone e Sluys (2011, 2010a, 2010b), Hettich, Hung e Ramm (2008), Chudoba, Jerábek e Peiffer (2009) e Oliver et al. (2008) enriquecimentos baseados no comportamento geral das ligações fibra-matriz são impostos no domínio bidimensional para modelar o acoplamento entre os meios e são baseados principalmente no método da partição da unidade (MELENK; BABUSKA, 2006; DUARTE; ODEN, 1996; ODEN; DUARTE; ZIENKIEWICZ, 1998; DUARTE; BABUSKA; ODEN, 2000; BABUSKA; MELENK, 1997). Estas formulações são muito elegantes e bem-postas, mas de difícil obtenção para o caso de fibras curvas, por exemplo. Outros desafios relacionados à estratégia de enriquecimento de campo incluem a consideração da transmissão direta de força fibra-fibra (RADTKE; SIMONE; SLUYS, 2010a) e a incorporação de reforços entrelaçados.

Exemplos de trabalhos que consideram a microestrutura do material são Schlangen e Van Mier (1992), Bolander Jr. e Saito (1997) e Li et al. (2006). 
Estratégias que adotam graus de liberdade com deslizamento para representar meios reforçados com fibras podem ser encontradas nos trabalhos de Balakrishnan e Murray (1986) e de Désir et al. (1999).

O presente estudo se preocupa com o comportamento geral de meios reforçados que desenvolvem grandes deslocamentos e, portanto, apresenta uma formulação alternativa para a análise não linear geométrica de domínios bidimensionais reforçados com fibras. Os elementos finitos bidimensionais utilizados para discretizar o contínuo são isoparamétricos de ordem qualquer. Para descrever a aproximação das fibras, primeiramente descreve-se um elemento reto de primeira ordem e, em seguida, os conceitos deste são generalizados para a obtenção do elemento curvo de ordem qualquer. A não linearidade geométrica é considerada consistentemente em ambos os casos. Os parâmetros nodais adotados são posições, e não deslocamentos, que são adequados para a modelagem de elementos curvos em grandes deslocamentos, devido à presença natural da regra da cadeia numérica (BONET et al., 2000; CODA; PACCOLA, 2007).

Os elementos de fibra são introduzidos na matriz usando relações cinemáticas nodais que garantem a aderência dos nós da fibra à matriz sem aumentar os graus de liberdade do sistema resultante e sem a necessidade de coincidência dos nós fibra-matriz na discretização (VANALLI, 2004; VANALLI; PACCOLA; CODA, 2008).

Em particular, Vanalli, Paccola e Coda (2008) observaram que uma ligação não conforme ocorre na interface fibra-matriz quando se acopla um elemento de fibra de primeira ordem com um elemento bidimensional de alta ordem. Para superar essa limitação, desenvolve-se um elemento de fibra de alta ordem que pode ser acoplado com elementos bidimensionais de ordem qualquer. Os resultados demonstram adesão total ao longo da interface fibra-contínuo. Quando se utiliza elementos de primeira ordem o procedimento é simples, mas a sua generalização para fibras de alta ordem requer cálculos adicionais.

Para apresentar a formulação desenvolvida este capítulo foi organizado em seções conforme descrito a seguir. Na seção 4.2 descreve-se o elemento bidimensional de ordem qualquer utilizado para discretizar a matriz. Nas seções 4.3 e 4.4 descrevem-se, respectivamente, o elemento de barra simples e o elemento curvo de ordem qualquer utilizados para discretizar as fibras. Na seção 4.5 apresenta-se a estratégia para introduzir as fibras na matriz sem aumentar os graus de liberdade e sem a necessidade de coincidência dos nós na discretização das fibras e da matriz. Na seção 4.6 descreve-se o procedimento matricial para o acoplamento fibra-matriz. Na seção 4.7 o procedimento para determinar a localização dos nós da fibra em um elemento bidimensional é descrito e, finalmente, na Seção 4.8 
apresentam-se os exemplos numéricos testados.

Para fazer distinção entre os diferentes meios considerados nos equacionamentos, adota-se o padrão simbólico ( $(\hat{)})$ para as variáveis e grandezas relacionadas ao elemento finito bidimensional de ordem qualquer; $(\bar{\bullet})$ para as variáveis e grandezas relacionadas ao elemento finito de fibra simples e ( $\tilde{\bullet})$ para as variáveis e grandezas relacionadas ao elemento finito de fibra curvo de ordem qualquer.

\subsection{CINEMÁTICA DO ELEMENTO FINITO BIDIMENSIONAL}

Seja $\Omega^{0}$ a configuração de referência natural indeformada de um corpo, $\Omega$ a configuração atual deformada e $\Omega^{l}$ uma configuração adimensional auxiliar (Figura 8).

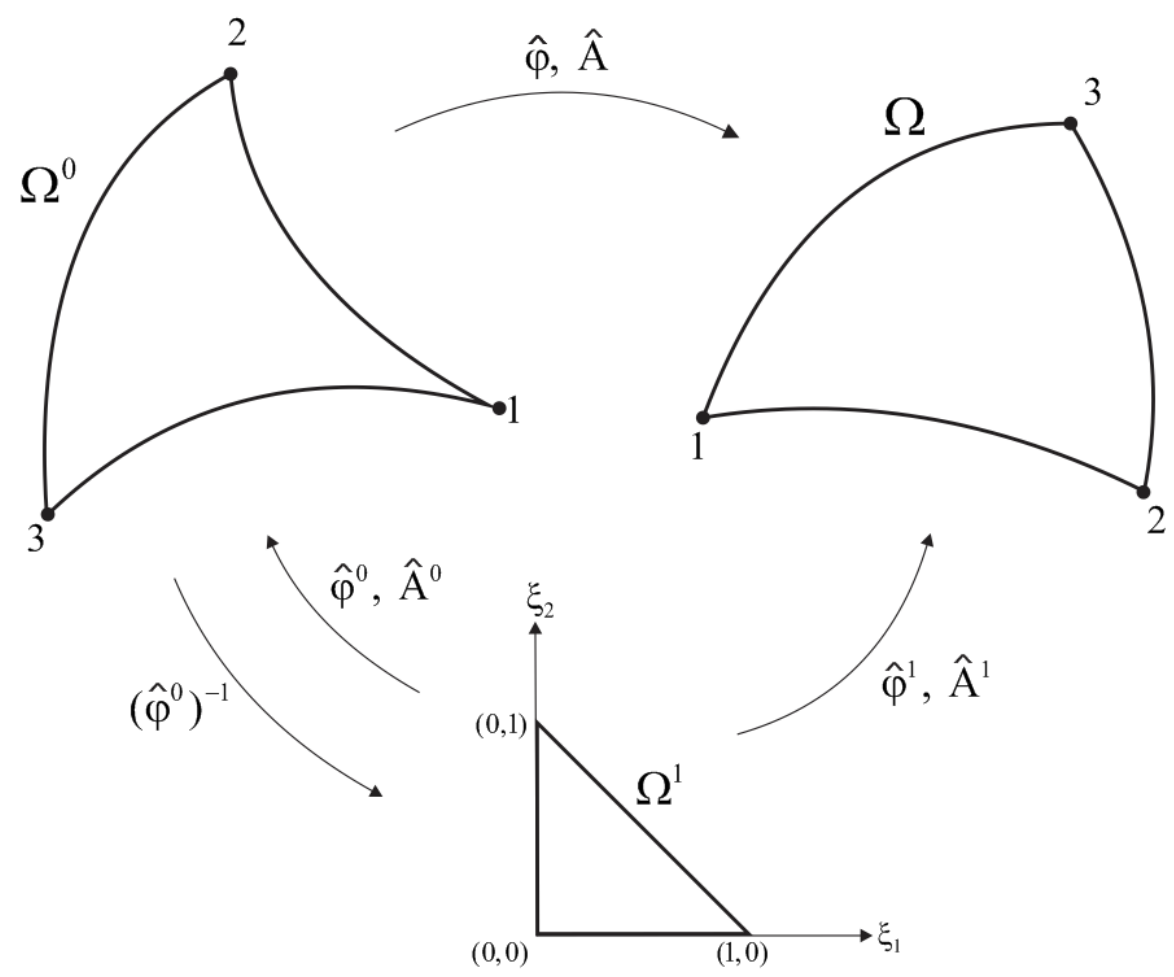

Figura 8 - Mapeamento do elemento bidimensional nas configurações inicial e final.

A configuração inicial $\Omega^{0}$ cujos pontos em seu domínio possuem coordenadas $\hat{x}_{i}$ é 
mapeada a partir do espaço adimensional $\Omega^{l}$ com coordenadas $\xi_{i}$ utilizando-se funções de forma de ordem qualquer, $\hat{\phi}_{l}\left(\xi_{1}, \xi_{2}\right)$, e as coordenadas dos nós $l$ na configuração inicial, $\hat{X}_{i}^{l}$, tal como:

$$
\hat{x}_{i}=\hat{\varphi}_{i}^{0}=\hat{\phi}_{l}\left(\xi_{1}, \xi_{2}\right) \hat{X}_{i}^{l}
$$

Analogamente, a configuração atual $\Omega$ é mapeada a partir do espaço adimensional $\Omega^{l}$ pela expressão:

$$
\hat{y}_{i}=\hat{\varphi}_{i}^{l}=\hat{\phi}_{l}\left(\xi_{1}, \xi_{2}\right) \hat{Y}_{i}^{l}
$$

onde $\hat{y}_{i}$ são as coordenadas dos pontos na configuração atual $\Omega$ e $\hat{Y}_{i}^{l}$ são as coordenadas atuais dos nós na configuração atual. Nas Eqs. (4.1)-(4.2) os índices $l=1, \ldots, n$ e $i=1,2$, correspondem, respectivamente, aos nós do elemento finito de chapa e aos graus de liberdade associados a estes nós.

A função mudança de configuração $\hat{\varphi}$ que mapeia os pontos com coordenadas $\hat{x}_{i}$ da configuração inicial $\Omega^{0}$ nos pontos com coordenadas $\hat{y}_{i}$ da configuração atual $\Omega$, em geral é desconhecida, mas pode ser escrita como uma composição dos mapeamentos $\hat{\varphi}^{0}$ que mapeia a configuração auxiliar $\Omega^{l}$ na configuração inicial $\Omega^{0}$ e $\hat{\varphi}^{l}$ que mapeia a configuração auxiliar $\Omega^{l}$ na configuração atual $\Omega$ pela expressão:

$$
\hat{\varphi}=\hat{\varphi}^{l} \circ\left(\hat{\varphi}^{0}\right)^{-1}
$$

Os gradientes dos mapeamentos $\hat{\varphi}^{0}$ e $\hat{\varphi}^{l}$, designados respectivamente por $\hat{A}^{0}$ e $\hat{A}^{l}$, compõem o gradiente $\hat{A}$ da função mudança de configuração $\hat{\varphi}$ e suas expressões são dadas, respectivamente, por:

$$
\hat{A}_{i j}^{o}=\frac{\partial \hat{\varphi}_{i}^{0}}{\partial \xi_{j}}, \hat{A}_{i j}^{l}=\frac{\partial \hat{\varphi}_{i}^{l}}{\partial \xi_{j}}, \hat{\boldsymbol{A}}=\hat{\boldsymbol{A}}^{l} \cdot\left(\hat{\boldsymbol{A}}^{0}\right)^{-1}
$$

Na Figura 8, o elemento finito bidimensional pode ter ordem de aproximação, $G P$, 
qualquer e o número total de nós do elemento, $\hat{N}$, pode ser determinado pela expressão:

$$
\hat{N}=\frac{(G P+1)(G P+2)}{2}
$$

\subsubsection{ENERGIA ESPECÍFICA DE DEFORMAÇÃO DO ELEMENTO BIDIMENSIONAL}

A energia específica de deformação empregada para o elemento finito bidimensional é a que descreve o material Saint-Venant-Kirchhoff e é dada por:

$$
\hat{w}=\frac{1}{2} \hat{\boldsymbol{E}}: \hat{\mathbb{C}}: \hat{\boldsymbol{E}} \text { ou } \hat{w}=\frac{1}{2} \hat{E}_{i j} \hat{\mathbb{C}}_{i j k l} \hat{E}_{k l},
$$

onde $\hat{C}_{i j k l}$ são as componentes do tensor de quarta ordem das constantes elásticas do material e $\hat{\boldsymbol{E}}$ é o tensor de segunda ordem das deformações de Green-Lagrange expressos, respectivamente, por:

$$
\begin{gathered}
\hat{\boldsymbol{C}}_{i j k l}=\frac{2 G v}{1-2 v} \delta_{i j} \delta_{k l}+G\left(\delta_{i k} \delta_{j l}+\delta_{i l} \delta_{j k}\right) \\
\hat{\boldsymbol{E}}=\frac{1}{2}(\hat{\boldsymbol{C}}-\hat{\boldsymbol{I}})=\frac{1}{2}\left(\hat{\boldsymbol{A}}^{T} \hat{\boldsymbol{A}}-\hat{\boldsymbol{I}}\right) \text { ou } \hat{E}_{i j}=\frac{1}{2}\left(\hat{C}_{i j}-\delta_{i j}\right)=\frac{1}{2}\left(\hat{A}_{k i} \hat{A}_{k j}-\delta_{i j}\right)
\end{gathered}
$$

Os tensores de segunda ordem $\hat{\boldsymbol{C}}=\hat{\boldsymbol{A}}^{T} \hat{\boldsymbol{A}}$ e $\hat{\boldsymbol{I}}$ na Eq. (4.8) são, respectivamente, o tensor dos estiramentos à direita de Cauchy-Green e o tensor delta de Kroenecker ou tensor Identidade.

Substituindo as Eqs. (4.7)-(4.8) na Eq. (4.6) resulta que a energia de deformação específica para Estado Plano de Deformação (EPD) e para Estado Plano de Tensão (EPT) são dadas, respectivamente, por:

$$
\begin{gathered}
\hat{w}=\frac{G}{1-2 v}\left\{(1-v)\left(E_{11}^{2}+E_{22}^{2}\right)+2 v E_{11} E_{22}+(1-2 v)\left(E_{12}^{2}+E_{21}^{2}\right)\right\}, \\
\hat{w}=\frac{G}{\left(1-v^{2}\right)}\left\{E_{11}^{2}+E_{22}^{2}+2 v E_{11} E_{22}+\left(1-v^{2}\right)\left(E_{12}^{2}+E_{21}^{2}\right)\right\}
\end{gathered}
$$


onde $G=\hat{\mathbb{E}} / 2(1+v)$ é o módulo de elasticidade transversal, $\hat{\mathbb{E}}$ é o módulo de Young e $v$ o coeficiente de Poisson.

\subsubsection{ENERGIA DE DEFORMAÇÃO DO ELEMENTO BIDIMENSIONAL}

A energia de deformação $\hat{W}$ acumulada em um elemento finito de chapa é calculada integrando-se a energia específica de deformação $\hat{w}$ dada na Eq. (4.6) no volume inicial $\hat{V}_{0}$ do elemento, ou seja,

$$
\hat{W}=\int_{\hat{V}_{0}} \hat{w} d \hat{V}_{0} .
$$

Substituindo-se a energia específica de deformação $\hat{w}$ escrita em função das coordenadas adimensionais $\xi_{1}$ e $\xi_{2}$ na Eq. (4.11) resulta:

$$
\hat{W}=\int_{0}^{1} \int_{0}^{1-\xi_{2}} \hat{w}\left(\xi_{1}, \xi_{2}\right) \hat{J}_{0}\left(\xi_{1}, \xi_{2}\right) d \xi_{1} d \xi_{2}
$$

onde $\hat{J}_{0}$ é o Jacobiano do mapeamento do espaço adimensional para a configuração inicial dado por:

$$
\hat{J}_{0}\left(\xi_{1}, \xi_{2}\right)=\operatorname{det}\left(\hat{\boldsymbol{A}}^{0}\right)
$$

com $\hat{\boldsymbol{A}}^{0}$ dado na Eq. (4.4).

A Eq. (4.12) é resolvida numericamente utilizando-se quadratura de Hammer. A integral é substituída por um somatório dos valores de $\hat{W}$ calculados em pontos préestabelecidos $\left(\xi_{1}, \xi_{2}\right)$ denominados pontos de Hammer, (nph), e multiplicados pelos respectivos pesos de integração, $\hat{c}_{i}$, ou seja,

$$
\hat{W}=\sum_{i=1}^{n p h} \hat{w}\left(\xi_{1}, \xi_{2}\right)_{i} \hat{c}_{i} \hat{J}_{0}\left(\xi_{1}, \xi_{2}\right)_{i}
$$


A energia de deformação acumulada em um meio bidimensional é calculada como a soma das energias de deformação acumuladas em todos os elementos finitos que constituem a discretização deste meio bidimensional.

\subsection{ELEMENTO FINITO DE BARRA SIMPLES}

Primeiramente, para simular trechos de fibra longa ou fibras curtas aleatórias inseridas no domínio bidimensional desenvolve-se um elemento de barra simples ou elemento de treliça com dois nós e dois graus de liberdade por nó.

A Figura 9 mostra um elemento finito de barra simples, $\bar{e}$, antes e depois da mudança de configuração. Os parâmetros nodais deste elemento são as posições $\bar{X}_{i}$, conhecidas, na configuração inicial e $\bar{Y}_{i}$, incógnitas, na configuração final, com $i=1,2$.

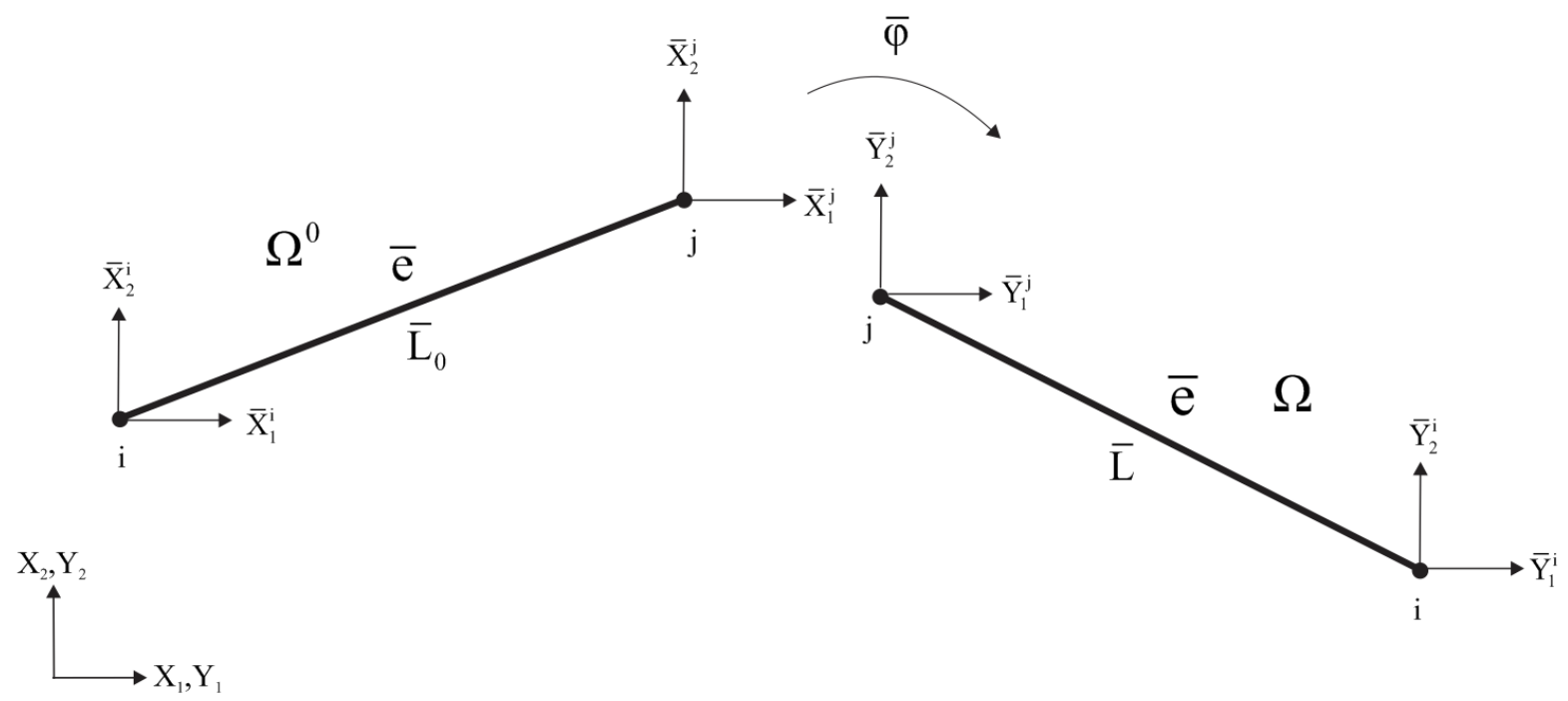

Figura 9 - Elemento finito de barra simples nas configurações inicial $\Omega^{0}$ e final $\Omega$.

Os comprimentos, inicial $\bar{L}_{0}$ e final $\bar{L}$, são obtidos a partir das posições dos nós do elemento finito por meio das expressões:

$$
\left(\bar{L}_{0}\right)^{2}=\left(\bar{X}_{1}^{j}-\bar{X}_{1}^{i}\right)^{2}+\left(\bar{X}_{2}^{j}-\bar{X}_{2}^{i}\right)^{2} e(\bar{L})^{2}=\left(\bar{Y}_{1}^{j}-\bar{Y}_{1}^{i}\right)^{2}+\left(\bar{Y}_{2}^{j}-\bar{Y}_{2}^{i}\right)^{2}
$$


Da mesma forma como para o elemento finito bidimensional adota-se o material de Saint-Venant-Kirchhoff cuja energia específica de deformação, para o elemento de barra simples, é dada por:

$$
\bar{w}=\frac{1}{2} \overline{\mathbb{E}} \bar{E}^{2},
$$

sendo $\overline{\mathbb{E}}$ o módulo elástico tangente que se confunde com o módulo de Young em pequenas deformações e $\bar{E}$ a medida de deformação de Green unidimensional definida a partir da diferença do quadrado do comprimento de uma fibra material do meio contínuo antes e depois da deformação, ou seja,

$$
\bar{E}=\frac{1}{2}\left(\frac{\bar{L}^{2}}{\bar{L}_{0}^{2}}-1\right),
$$

Integrando a energia específica de deformação $\bar{w}$ dada pela Eq. (4.16) no volume inicial $\bar{V}_{0}$ do elemento finito de barra simples tem-se que a energia de deformação $\bar{W}$ acumulada no elemento finito é dada por:

$$
\bar{W}=\int_{\bar{V}_{o}} \bar{w} d \bar{V}_{o}=\frac{1}{2} \overline{\mathbb{E}} \bar{E}^{2} \bar{V}_{0},
$$

sendo $\bar{V}_{0}=\bar{A} \bar{L}_{0}$ e $\bar{A}$ a área da seção transversal da fibra na configuração inicial, resultando da Eq. (4.18) que a energia de deformação é dada por:

$$
\bar{W}=\frac{1}{2} \overline{\mathbb{E}} \bar{E}^{2} \bar{A} \bar{L}_{0}
$$

O vetor de forças internas $\bar{F}_{i}^{\text {int }}$ definido como a primeira derivada da energia de deformação em relação aos parâmetros nodais do elemento finito considerado e a matriz Hessiana $\bar{H}_{i j}$ definida como a segunda derivada da energia de deformação em relação aos parâmetros nodais do elemento finito considerado são dados, respectivamente, por: 


$$
\begin{gathered}
\bar{F}_{i}{ }^{i n t}=\frac{\partial \bar{W}_{i}}{\partial \bar{Y}_{i}}=\frac{\partial}{\partial \bar{Y}_{i}}\left(\frac{1}{2} \bar{E}_{E} \bar{E}^{2} \bar{A} \bar{L}_{0}\right)=\frac{1}{2} \bar{E} \bar{E} \bar{E} \bar{L}_{0} \frac{\partial \bar{E}}{\partial \bar{Y}_{i}}, \\
\bar{H}_{i j}=\frac{\partial \bar{F}_{i}^{i n t}}{\partial \bar{Y}_{j}}=\frac{\partial^{2} \bar{W}}{\partial \bar{Y}_{j} \partial \bar{Y}_{i}}=\frac{\partial}{\partial \bar{Y}_{j}}\left(\frac{1}{2} \overline{\mathbb{E}} \overline{E A} \bar{L}_{0} \frac{\partial \bar{E}}{\partial \bar{Y}_{i}}\right) .
\end{gathered}
$$

As expressões das componentes do vetor de forças internas, Eq. (4.20), e da matriz Hessiana, Eq. (4.21), do elemento finito de fibra simples são obtidos utilizando-se as Eqs. (4.17) e (4.15) e são apresentadas no APÊNDICE A.

\subsection{ELEMENTO FINITO CURVO DE ORDEM QUALQUER}

Quando o elemento de fibra simples, reto e com aproximação linear é adotado, a aderência entre fibra e matriz ocorre através dos nós e a condição de aderência entre os contínuos será satisfatória com um número muito grande de elementos de fibra.

Para melhorar este comportamento, desenvolve-se um elemento finito de fibra curvo de ordem qualquer e, no final dos desenvolvimentos deste capítulo, mostrar-se-á que para elementos de fibra com mesma ordem de aproximação que os elementos de chapa, a aderência entre os meios estará plenamente satisfeita.

Desta forma, descreve-se, nesta seção, o elemento finito curvo de ordem qualquer utilizado para simular trechos de fibras longas e fibras curtas aleatórias.

\subsubsection{CINEMÁTICA DO ELEMENTO FINITO CURVO DE ORDEM QUALQUER}

A Figura 10 mostra um elemento finito de fibra curvo de ordem qualquer antes e depois da mudança de configuração. Da mesma forma como para o elemento de barra simples os parâmetros nodais deste elemento são as posições $\tilde{X}_{i}$ na configuração inicial e $\tilde{Y}_{i}$ na configuração final.

Analogamente ao descrito para o elemento de chapa apresentado na Seção 4.2, considera-se $\Omega^{0}$ uma configuração de referência natural indeformada, $\Omega$ uma configuração 
atual deformada e $\Omega^{l}$ uma configuração auxiliar adimensional (Figura 10).

A configuração inicial $\Omega^{0}$, cujos pontos em seu domínio possuem coordenadas $\tilde{x}_{i}$, é mapeada a partir do espaço adimensional $\Omega^{1}$ com coordenada $\eta$ utilizando-se funções de forma de ordem qualquer, $\tilde{\phi}_{p}(\eta)$, e das coordenadas dos nós $p$ do elemento de fibra na configuração inicial, $\tilde{X}_{i}^{p}$, tal como:

$$
\tilde{x}_{i}=\tilde{\varphi}_{i}^{0}=\tilde{\phi}_{p}(\eta) \tilde{X}_{i}^{p}
$$

A configuração atual $\Omega$ é mapeada a partir do espaço adimensional $\Omega^{l}$ pela expressão:

$$
\tilde{y}_{i}=\tilde{\varphi}_{i}^{l}=\tilde{\phi}_{p}(\eta) \tilde{Y}_{i}^{p}
$$

onde $\tilde{y}_{i}$ são coordenadas dos pontos na configuração atual $\Omega$ e $\tilde{Y}_{i}^{p}$ são as coordenadas atuais dos nós do elemento finito de fibra na configuração atual $\Omega$. Nas Eqs. (4.22)-(4.23) os índices $p=1, \ldots, n$ e $i=1,2$, correspondem, respectivamente, aos nós do elemento finito de fibra curvo e aos graus de liberdade associados a estes nós.
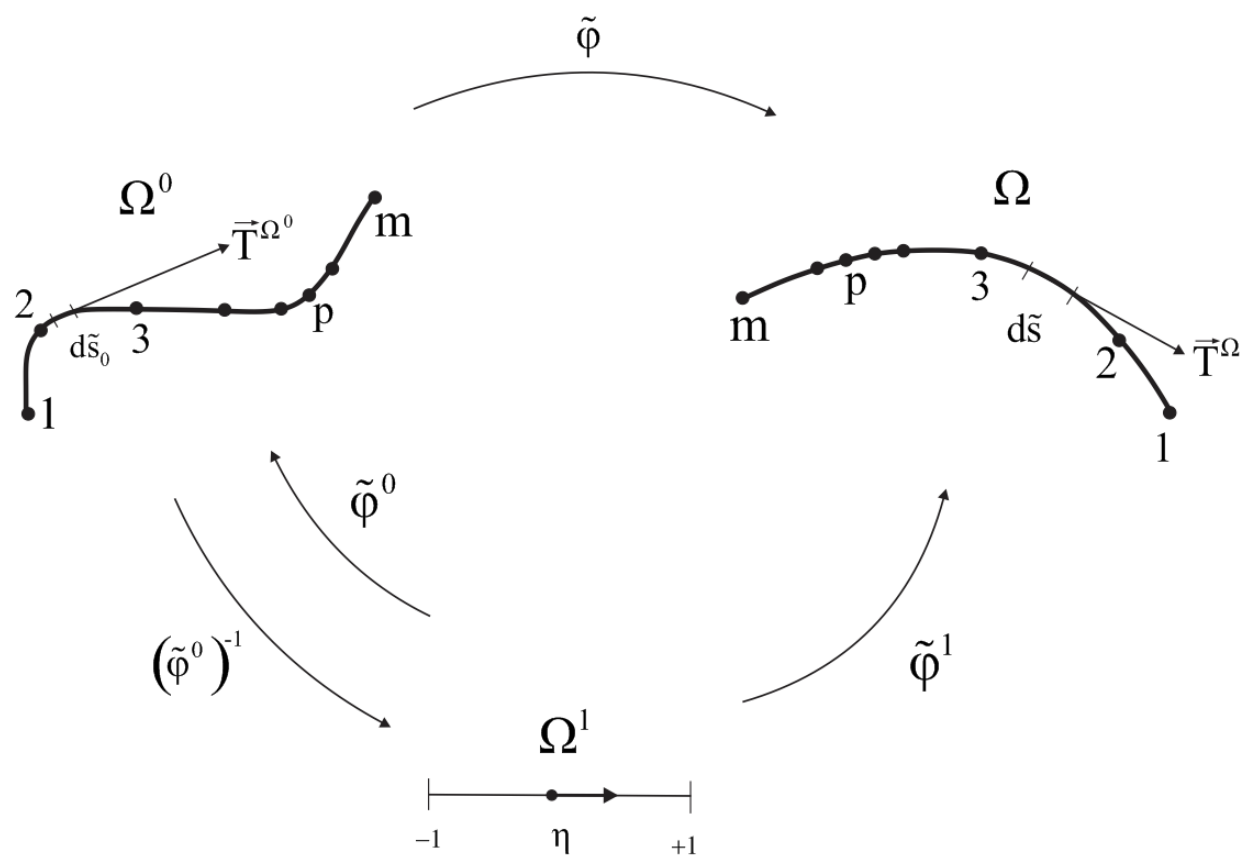

$$
+1
$$

Figura 10 - Mapeamento do elemento de fibra curvo de ordem qualquer nas configurações inicial e final. 
A cada ponto do elemento finito de fibra curva na configuração inicial $\Omega^{0}$ existe um vetor tangente associado expresso, juntamente com seu módulo, por:

$$
T_{i}^{\Omega^{0}}=\frac{d \tilde{\phi}_{p}(\eta)}{d \eta} \tilde{X}_{i}^{p} \text { e }\left|\vec{T}^{\Omega^{0}}\right|^{2}=\left(\frac{d \tilde{\phi}_{p}(\eta)}{d \eta} \tilde{X}_{1}^{p}\right)^{2}+\left(\frac{d \tilde{\phi}_{p}(\eta)}{d \eta} \tilde{X}_{2}^{p}\right)^{2}
$$

Analogamente, o vetor tangente associado aos nós do elemento finito de fibra curvo na configuração atual $\Omega$ e seu módulo são dados, respectivamente, por:

$$
T_{i}^{\Omega}=\frac{d \tilde{\phi}_{p}(\eta)}{d \eta} \tilde{Y}_{i}^{p} \text { e }\left|\vec{T}^{\Omega}\right|^{2}=\left(\frac{d \tilde{\phi}_{p}(\eta)}{d \eta} \tilde{Y}_{1}^{p}\right)^{2}+\left(\frac{d \tilde{\phi}_{p}(\eta)}{d \eta} \tilde{Y}_{2}^{p}\right)^{2}
$$

A partir dos módulos dos vetores tangentes nas configurações inicial e atual dados nas Eqs. (4.24) e (4.25) define-se a medida de deformação de Green $\tilde{E}$ associada aos nós do elemento finito de fibra curvo, como:

$$
\tilde{E}=\frac{1}{2}\left(\frac{\left|\vec{T}^{\Omega}\right|^{2}-\left|\vec{T}^{\Omega^{0}}\right|^{2}}{\left|\vec{T}^{\Omega^{0}}\right|^{2}}\right),
$$

que em sua forma expandida é reescrita como:

$$
\tilde{E}=\frac{1}{2} \frac{\left[\left(\frac{d \tilde{\phi}_{p}(\eta)}{d \eta} \tilde{Y}_{1}^{p}\right)^{2}+\left(\frac{d \tilde{\phi}_{p}(\eta)}{d \eta} \tilde{Y}_{2}^{p}\right)^{2}\right]-\left[\left(\frac{d \tilde{\phi}_{p}(\eta)}{d \eta} \tilde{X}_{1}^{p}\right)^{2}+\left(\frac{d \tilde{\phi}_{p}(\eta)}{d \eta} \tilde{X}_{2}^{p}\right)^{2}\right]}{\left(\frac{d \tilde{\phi}_{p}(\eta)}{d \eta} \tilde{X}_{1}^{p}\right)^{2}+\left(\frac{d \tilde{\phi}_{p}(\eta)}{d \eta} \tilde{X}_{2}^{p}\right)^{2}} .
$$

Da mesma forma como para o elemento finito de barra simples, adota-se a Lei Constitutiva de Saint-Venant-Kirchhoff uniaxial para descrever a energia específica $\tilde{w}$ de deformação do elemento finito de fibra curvo de ordem qualquer, ou seja,

$$
\tilde{w}=\frac{1}{2} \tilde{\mathbb{E}} \tilde{E}^{2},
$$


sendo $\tilde{\mathbb{E}}$ o módulo elástico tangente e $\tilde{E}$ a medida de deformação de Green definida na Eq. (4.27).

Lembrando-se que a energia de deformação $\tilde{W}$ acumulada em um elemento finito de fibra curvo pode ser obtida integrando-se a energia específica de deformação $\tilde{w}$ dada na Eq. (4.28)no volume inicial $\tilde{V}_{0}$ da mesma, tem-se:

$$
\tilde{W}=\int_{\tilde{V}_{0}} \tilde{w} d \tilde{V}_{0} .
$$

As componentes do vetor de forças internas para o elemento finito de fibra curvo de ordem qualquer são definidas como:

$$
\tilde{F}_{k}^{j}=\frac{\partial \tilde{W}}{\partial \tilde{Y}_{k}^{j}}=\int_{\tilde{V}_{o}} \frac{\partial \tilde{w}}{\partial \tilde{Y}_{k}^{j}} d \tilde{V}_{o}
$$

Das Eqs. (4.28) e (4.27) resulta da Eq. (4.30) que:

$$
\tilde{F}_{k}^{j}=\frac{\partial \tilde{W}}{\partial \tilde{Y}_{k}^{j}}=\int_{\tilde{V}_{o}} \tilde{\mathbb{E}} \tilde{E} \frac{\left(\frac{d \tilde{\phi}_{p}(\eta)}{d \eta} Y_{k}^{p}\right) \frac{d \tilde{\phi}_{j}(\eta)}{d \eta}}{\left|\vec{T}^{\Omega^{0}}\right|^{2}} d \tilde{V}_{0}
$$

sendo $\tilde{V}_{0}=\tilde{A} \tilde{L}_{0}$ e $\tilde{A}$ a área da seção transversal da fibra na configuração inicial. Integrando-se a Eq. (4.31) no volume inicial do elemento de fibra curvo de ordem qualquer e mudando-se os limites de integração para o intervalo $[-1,+1]$, resulta, respectivamente em:

$$
\tilde{F}_{k}^{j}=\frac{\partial \tilde{W}}{\partial \tilde{Y}_{k}^{j}}=\int_{0}^{\tilde{L}_{0}} \tilde{\mathbb{E}} \tilde{E} \frac{\left(\frac{d \tilde{\phi}_{p}(\eta)}{d \eta} \tilde{Y}_{k}^{p}\right) \frac{d \tilde{\phi}_{j}(\eta)}{d \eta}}{\left|\vec{T}^{\Omega^{0}}\right|^{2}} \tilde{A} d \tilde{s}_{0}=\int_{-1}^{1} \tilde{E} \tilde{E} \frac{\left(\frac{d \tilde{\phi}_{p}(\eta)}{d \eta} \tilde{Y}_{k}^{p}\right) \frac{d \tilde{\phi}_{j}(\eta)}{d \eta}}{\left|\vec{T}^{\Omega^{0}}\right|^{2}} \tilde{J}_{0}(\eta) \tilde{A} d \eta \cdot
$$

onde $\tilde{J}_{0}(\eta)$ é o Jacobiano do mapeamento do espaço adimensional $\Omega^{1}$ para a configuração inicial $\Omega^{0}$ dado para o caso do elemento de fibra curvo de ordem qualquer por: 


$$
\tilde{J}_{0}(\eta)=\left|\vec{T}^{\Omega^{0}}\right|=\sqrt{\left(\frac{d \tilde{x}_{1}}{d \eta}\right)^{2}+\left(\frac{d \tilde{x}_{2}}{d \eta}\right)^{2}}
$$

onde $\left|\vec{T}^{\Omega^{0}}\right|$ é o módulo do vetor tangente definido na configuração inicial dado na Eq. (4.24).

A Eq. (4.32) é resolvida numericamente utilizando-se quadratura de Gauss-Legendre. A integral é substituída por um somatório dos valores do integrando calculados em pontos pré-estabelecidos $(\eta)$ denominados pontos de Gauss, ( $n p g$ ), e multiplicados pelos respectivos pesos de integração, $\tilde{c}_{i}$, ou seja,

$$
\tilde{F}_{k}^{j}=\frac{\partial \tilde{W}}{\partial \tilde{Y}_{k}^{j}}=\left.\sum_{i=1}^{n p g} \frac{\tilde{E} \tilde{E}}{\left|\vec{T}^{\Omega^{0}}\right|^{2}}\left(\left.\frac{d \tilde{\phi}_{p}(\eta)}{d \eta}\right|_{\eta=\eta_{i}} \tilde{Y}_{k}^{p}\right) \frac{d \tilde{\phi}_{j}(\eta)}{d \eta}\right|_{\eta=\eta_{i}} \tilde{c}_{i} \tilde{J}_{0}\left(\eta_{i}\right) \tilde{A}
$$

As componentes $\tilde{H}_{k j \alpha \beta}$ da matriz Hessiana do elemento de fibra curvo de ordem qualquer são obtidas por meio da expressão:

$$
\tilde{H}_{k j \alpha \beta}=\frac{\partial^{2} \tilde{W}}{\partial \tilde{Y}_{k}^{j} \partial \tilde{Y}_{\alpha}^{\beta}}=\int_{\tilde{V}_{0}} \frac{\partial^{2} \tilde{w}}{\partial \tilde{Y}_{k}^{j} \partial \tilde{Y}_{\alpha}^{\beta}} d \tilde{V}_{o}
$$

Analogamente ao realizado para o vetor de forças internas resulta das Eqs. (4.28), (4.27) e (4.35) que:

$$
\tilde{H}_{k j \alpha \beta}=\int_{\tilde{V}_{o}}\left(\frac{\tilde{\mathbb{E}}}{\left|\vec{T}^{\Omega^{0}}\right|^{4}}\left(\frac{d \tilde{\phi}_{p}(\eta)}{d \eta} \tilde{Y}_{\alpha}^{p}\right) \frac{d \tilde{\phi}_{\beta}(\eta)}{d \eta}\left(\frac{d \tilde{\phi}_{p}(\eta)}{d \eta} \tilde{Y}_{k}^{p}\right) \frac{d \tilde{\phi}_{j}(\eta)}{d \eta}+\frac{\tilde{\mathbb{E}} \tilde{E}}{\left|\vec{T}^{\Omega^{0}}\right|^{2}} \frac{d \tilde{\phi}_{\beta}(\eta)}{d \eta} \frac{d \tilde{\phi}_{j}(\eta)}{d \eta} \delta_{k \alpha}\right) d \tilde{V}_{0},
$$

ou ainda,

$$
\tilde{H}_{k j \alpha \beta}=\int_{0}^{\tilde{L}_{0}}\left(\frac{\tilde{\mathbb{E}}}{\left|\vec{T}^{\Omega^{0}}\right|^{4}}\left(\frac{d \tilde{\phi}_{p}(\eta)}{d \eta} \tilde{Y}_{\alpha}^{p}\right) \frac{d \tilde{\phi}_{\beta}(\eta)}{d \eta}\left(\frac{d \tilde{\phi}_{p}(\eta)}{d \eta} \tilde{Y}_{k}^{p}\right) \frac{d \tilde{\phi}_{j}(\eta)}{d \eta}+\frac{\tilde{\mathbb{E}} \tilde{E}}{\left|\vec{T}^{\Omega^{o}}\right|^{2}} \frac{d \tilde{\phi}_{\beta}(\eta)}{d \eta} \frac{d \tilde{\phi}_{j}(\eta)}{d \eta} \delta_{k \alpha}\right) \tilde{A}_{d} \tilde{s}_{0}
$$

Mudando-se os limites de integração da Eq. (4.37) para o intervalo $[-1,+1]$ tem-se que: 


$$
\tilde{H}_{k j \alpha \beta}=\int_{-I}^{l}\left(\frac{\tilde{E}}{\left|\vec{T}^{\Omega^{0}}\right|^{4}}\left(\frac{d \tilde{\phi}_{p}(\eta)}{d \eta} \tilde{Y}_{\alpha}^{p}\right) \frac{d \tilde{\phi}_{\beta}(\eta)}{d \eta}\left(\frac{d \tilde{\phi}_{p}(\eta)}{d \eta} \tilde{Y}_{k}^{p}\right) \frac{d \tilde{\phi}_{j}(\eta)}{d \eta}+\frac{\tilde{E} \tilde{E}}{\left|\vec{T}^{\Omega^{0}}\right|^{2}} \frac{d \tilde{\phi}_{\beta}(\eta)}{d \eta} \frac{d \tilde{\phi}_{j}(\eta)}{d \eta} \delta_{k \alpha}\right) \tilde{J}_{o}(\eta) \tilde{A} d \eta
$$

Para a resolução numérica utilizando quadratura de Gauss-Legendre a Eq. (4.38) é reescrita convenientemente como:

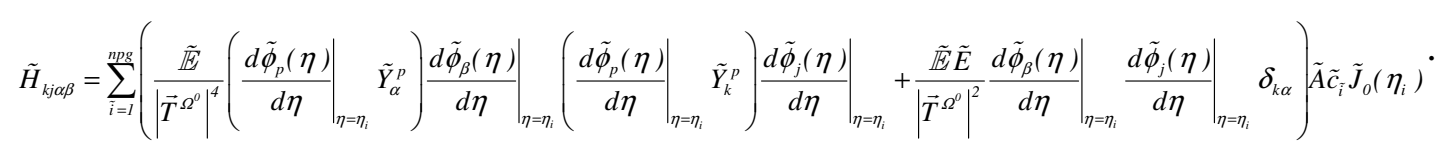

Os elementos finitos de fibra curvos de ordem qualquer são gerados utilizando-se os polinômios de Lagrange apresentados no ANEXO A.

\subsection{ACOPLAMENTO FIBRA-MATRIZ}

Da forma como apresentado até agora, os vetores de força interna e matriz Hessiana do elemento de chapa e de fibra foram obtidos independentemente. Nesta seção, as derivadas são desenvolvidas considerando-se as contribuições dos dois meios simultaneamente.

O procedimento proposto para inserir as fibras em uma posição qualquer do domínio sem aumentar o número de graus de liberdade do sistema de equações resultante e sem a necessidade de coincidência dos nós na discretização das fibras e da matriz segue a ideia inicial apresentada por Vanalli (2004) para a análise de problemas lineares geométricos e consiste em escrever as posições dos nós dos elementos finitos de fibra em função das posições dos nós dos elementos finitos de chapa onde as fibras estão imersas. Na configuração inicial se escreve as posições $\bar{X}_{i}$ dos nós das fibras retas e as posições $\tilde{X}_{i}$ dos nós das fibras curvas, respectivamente, como:

$$
\bar{X}_{i}=\hat{\phi}_{l}\left(\xi_{1}^{p}, \xi_{2}^{p}\right) \hat{X}_{i}^{l} \text { e } \tilde{X}_{i}=\hat{\phi}_{l}\left(\xi_{1}^{p}, \xi_{2}^{p}\right) \hat{X}_{i}^{l}
$$

onde $\hat{\phi}_{l}$ são as funções de forma do elemento finito de chapa calculadas para as coordenadas adimensionais $\xi_{i}^{p}$ do nó $p$ do elemento de fibra considerado e $\hat{X}_{i}^{l}$ são as posições dos nós 
da chapa na configuração inicial. Na configuração atual $\Omega$, as posições $\bar{Y}_{i}$ e $\tilde{Y}_{i}$ dos nós das fibras retas e curvas, respectivamente, podem ser obtidas a partir das expressões:

$$
\bar{Y}_{i}=\hat{\phi}_{l}\left(\xi_{1}^{p}, \xi_{2}^{p}\right) \hat{Y}_{i}^{l} \text { e } \tilde{Y}_{i}=\hat{\phi}_{l}\left(\xi_{1}^{p}, \xi_{2}^{p}\right) \hat{Y}_{i}^{l}
$$

sendo $\hat{Y}_{i}^{l}$ as posições atuais dos nós da chapa. A Eq. (4.41) garante a aderência dos nós das fibras retas ou curvas à matriz. Para o caso de fibras retas, considerando que a quantidade de elementos de fibra pode ser qualquer sem o aumento do número de incógnitas, a condição de aderência entre o domínio e as fibras pode ser estendida ao longo da superfície de contato quando o número de elementos finitos aumenta.

\subsubsection{ENERGIA DE DEFORMAÇÃO DE DOMÍNIOS ELÁSTICOS BIDIMENSIONAIS REFORÇADOS COM FIBRAS - FORÇA INTERNA}

A energia de deformação $W$ armazenada em um corpo reforçado por fibras é a soma das energias de deformação armazenadas na matriz e na fibra. Nesta seção, a obtenção do vetor de forças internas é feita considerando-se que a matriz é reforçada com fibras retas. Para matriz reforçada com fibras curvas o equacionamento é análogo ao descrito para a fibra reta. Desta forma a energia $W$ armazenada em um corpo reforçado por fibras retas é escrita como:

$$
W=\hat{W}+\bar{W}
$$

onde $\hat{W}$ é a energia de deformação armazenada nos elementos finitos bidimensionais utilizados para discretizar a matriz e $\bar{W}$ é a energia de deformação armazenada nos elementos de fibra reta. Portanto, a força interna na direção $\alpha$ em um nó $\beta$ de um elemento finito de chapa considerando a contribuição da fibra é encontrada como:

$$
\frac{\partial(\hat{W}+\bar{W})}{\partial \hat{Y}_{\alpha}^{\beta}}=F_{\alpha}^{\beta i n t} .
$$

Substituindo-se a expressão da energia de deformação do elemento de chapa e de fibra 
escrita em função das posições nodais na Eq. (4.42) tem-se:

$$
W=\int_{\hat{V}_{0}} \hat{w} d \hat{V}_{0}+\int_{\bar{V}_{0}} \bar{w}\left(\bar{Y}_{j}\right) d \bar{V}_{o}
$$

Observa-se das Eqs. (4.41) e (4.44) que a energia de deformação das fibras, $\bar{w}$, é função das coordenadas dos nós da chapa, ou seja,

$$
W=\int_{\hat{V}_{0}} \hat{w} d \hat{V}_{0}+\int_{\bar{V}_{0}} \bar{w}\left(\bar{Y}_{j}\left(\hat{Y}_{i}^{l}\right)\right) d \bar{V}_{0}
$$

Substituindo a Eq. (4.45) na Eq. (4.43) resulta:

$$
F_{\alpha}^{\beta i n t}=\frac{\partial W}{\partial \hat{Y}_{\alpha}^{\beta}}=\int_{\hat{V}_{o}} \frac{\partial \hat{w}}{\partial \hat{Y}_{\alpha}^{\beta}} d \hat{V}_{o}+\int_{\bar{V}_{o}} \frac{\partial \bar{w}}{\partial \hat{Y}_{\alpha}^{\beta}}\left(\bar{Y}_{j}\left(\hat{Y}_{i}^{l}\right)\right) d \bar{V}_{0}
$$

A derivada da energia específica de deformação do elemento de chapa $\hat{w}$ em relação aos seus parâmetros nodais na Eq. (4.46) é dada por:

$$
\frac{\partial \hat{w}}{\partial \hat{Y}_{\alpha}^{\beta}}=\frac{\partial \hat{w}}{\partial \hat{\boldsymbol{E}}}: \frac{\partial \hat{\boldsymbol{E}}}{\partial \hat{\boldsymbol{C}}}: \frac{\partial \hat{\boldsymbol{C}}}{\partial \hat{Y}_{\alpha}^{\beta}}=\frac{1}{2} \frac{\partial \hat{w}}{\partial \hat{\boldsymbol{E}}}: \frac{\partial \hat{\boldsymbol{C}}}{\partial \hat{Y}_{\alpha}^{\beta}}
$$

onde $\hat{\boldsymbol{S}}=\frac{\partial \hat{w}}{\partial \hat{\boldsymbol{E}}}$ é o tensor das tensões de Piola-Kirchhoff de segunda espécie, $\hat{w}$ é dada pela Eq. (4.9) ou Eq. (4.10) e

$$
\frac{\partial \hat{\boldsymbol{C}}}{\partial \hat{Y}_{\alpha}^{\beta}}=\left(\hat{\boldsymbol{A}}^{0}\right)^{-T} \cdot \frac{\partial\left(\hat{\boldsymbol{A}}^{l}\right)^{T}}{\partial \hat{Y}_{\alpha}^{\beta}} \cdot \hat{\boldsymbol{A}}^{1} \cdot\left(\hat{\boldsymbol{A}}^{0}\right)^{-1}+\left(\hat{\boldsymbol{A}}^{0}\right)^{-T} \cdot\left(\hat{\boldsymbol{A}}^{1}\right)^{T} \cdot \frac{\partial \hat{\boldsymbol{A}}^{l}}{\partial \hat{Y}_{\alpha}^{\beta}} \cdot\left(\hat{\boldsymbol{A}}^{0}\right)^{-1}
$$

sendo $\hat{A}_{i j}^{l}=\left(\hat{\phi}_{l, j}\left(\xi_{1}, \xi_{2}\right) \hat{Y}_{i}^{l}\right)$ e portanto $\frac{\partial \hat{A}_{i j}^{l}}{\partial \hat{Y}_{\alpha}^{\beta}}=\left(\hat{\phi}_{l, j}\left(\xi_{1}, \xi_{2}\right) \frac{\partial \hat{Y}_{i}^{l}}{\partial \hat{Y}_{\alpha}^{\beta}}\right) \operatorname{com} \frac{\partial \hat{Y}_{i}^{l}}{\partial \hat{Y}_{\alpha}^{\beta}}=\delta_{\alpha i} \delta_{\beta l}$.

A derivada da energia específica de deformação $\bar{w}$ do elemento de fibra reta em relação aos parâmetros nodais da chapa, na Eq. (4.46), é feita aplicando-se a regra da cadeia, como:

$$
\frac{\partial \bar{w}}{\partial \hat{Y}_{\alpha}^{\beta}}=\frac{\partial \bar{w}}{\partial \bar{Y}_{i}^{p}} \frac{\partial \bar{Y}_{i}^{p}}{\partial \hat{Y}_{\alpha}^{\beta}}
$$


sendo $\frac{\partial \bar{w}}{\partial \bar{Y}_{i}^{p}}$ o vetor gradiente da energia de deformação ou o vetor de forças internas da fibra descrito na Eq. (4.20) para a fibra reta e na Eq. (4.34) para a fibra curva. Finalmente se escreve $\frac{\partial \bar{Y}_{i}^{p}}{\partial \hat{Y}_{\alpha}^{\beta}}=\hat{\phi}^{\beta}\left(\xi_{1}^{p}, \xi_{2}^{p}\right)$ se $\alpha=i$.

\subsubsection{ENERGIA DE DEFORÇÃO DE DOMÍNIOS ELÁSTICOS BIDIMENSIONAIS REFORÇADOS COM FIBRAS - MATRIZ HESSIANA}

Procedendo-se conforme descrito para o cálculo das forças internas, realiza-se a segunda derivada da energia de deformação armazenada em um corpo reforçado por fibras retas em relação aos parâmetros nodais da matriz. Assim, tem-se que:

$$
H_{\alpha \beta \gamma_{\xi}^{\xi}}=\frac{\partial^{2} W}{\partial \hat{Y}_{\alpha}^{\beta} \partial \hat{Y}_{\gamma}^{\xi}}=\int_{\hat{V}_{0}} \frac{\partial^{2} \hat{w}}{\partial \hat{Y}_{\alpha}^{\beta} \partial \hat{Y}_{\gamma}^{\xi}} d \hat{V}_{o}+\int_{\bar{V}_{0}} \frac{\partial^{2} \bar{w}}{\partial \hat{Y}_{\alpha}^{\beta} \partial \hat{Y}_{\gamma}^{\xi}}\left(\bar{Y}_{j}\left(\hat{Y}_{i}^{l}\right)\right) d \bar{V}_{o}
$$

A segunda derivada da energia de deformação do elemento finito de chapa em relação aos seus parâmetros nodais, $\frac{\partial^{2} \hat{w}}{\partial \hat{Y}_{\alpha}^{\beta} \partial \hat{Y}_{\gamma}^{\xi}}$ na Eq. (4.50), é dada por:

$$
\frac{\partial^{2} \hat{w}}{\partial \hat{Y}_{\alpha}^{\beta} \partial \hat{Y}_{\gamma}^{\xi}}=\frac{1}{4} \frac{\partial^{2} \hat{w}}{\partial \hat{\boldsymbol{E}} \partial \hat{\boldsymbol{E}}}: \frac{\partial \hat{\boldsymbol{C}}}{\partial \hat{Y}_{\gamma}^{\xi}}: \frac{\partial \hat{\boldsymbol{C}}}{\partial \hat{Y}_{\alpha}^{\beta}}+\frac{1}{2} \frac{\partial \hat{w}}{\partial \hat{\boldsymbol{E}}}: \frac{\partial^{2} \hat{\boldsymbol{C}}}{\partial \hat{Y}_{\gamma}^{\xi} \partial \hat{Y}_{\alpha}^{\beta}}
$$

sendo

$$
\begin{aligned}
\frac{\partial^{2} \hat{\boldsymbol{C}}}{\partial \hat{Y}_{\alpha}^{\beta} \partial \hat{Y}_{\gamma}^{\xi}=} & \left(\hat{\boldsymbol{A}}^{0}\right)^{-T} \cdot \frac{\partial^{2}\left(\hat{\boldsymbol{A}}^{1}\right)^{T}}{\partial \hat{Y}_{\alpha}^{\beta} \partial \hat{Y}_{\gamma}^{\xi}} \cdot \hat{\boldsymbol{A}}^{1} \cdot\left(\hat{\boldsymbol{A}}^{0}\right)^{-1}+\left(\hat{\boldsymbol{A}}^{0}\right)^{-T} \cdot \frac{\partial\left(\hat{\boldsymbol{A}}^{1}\right)^{T}}{\partial \hat{Y}_{\alpha}^{\beta}} \cdot \frac{\partial \hat{\boldsymbol{A}}^{1}}{\partial \hat{Y}_{\gamma}^{\xi}} \cdot\left(\hat{\boldsymbol{A}}^{0}\right)^{-1}+ \\
& \left(\hat{\boldsymbol{A}}^{0}\right)^{-T} \cdot \frac{\partial\left(\hat{\boldsymbol{A}}^{1}\right)^{T}}{\partial \hat{Y}_{\gamma}^{\xi}} \cdot \frac{\partial \hat{\boldsymbol{A}}^{1}}{\partial \hat{Y}_{\alpha}^{\beta}} \cdot\left(\hat{\boldsymbol{A}}^{0}\right)^{-1}+\left(\hat{\boldsymbol{A}}^{0}\right)^{-T} \cdot\left(\hat{\boldsymbol{A}}^{1}\right)^{T} \cdot \frac{\partial^{2} \hat{\boldsymbol{A}}^{1}}{\partial \hat{Y}_{\alpha}^{\beta} \partial \hat{Y}_{\gamma}^{\xi}} \cdot\left(\hat{\boldsymbol{A}}^{0}\right)^{-1}
\end{aligned}
$$

Observa-se da Eq. (4.52) que a segunda derivada de $\hat{\boldsymbol{A}}^{l}$ em relação aos parâmetros nodais é nula para elementos bidimensionais. Elementos de casca não apresentam esta característica (CODA; PACCOLA, 2008).

A segunda derivada da energia de deformação da fibra reta em relação aos parâmetros 
nodais da chapa, $\frac{\partial^{2} \bar{w}}{\partial \hat{Y}_{\alpha}^{\beta} \partial \hat{Y}_{\gamma}^{\xi}}$ na Eq. (4.50), resulta em:

$$
\begin{aligned}
\frac{\partial^{2} \bar{w}}{\partial \hat{Y}_{\alpha}^{\beta} \partial \hat{Y}_{\gamma}^{\xi}=} & \frac{\partial^{2} \bar{w}}{\partial \bar{Y}_{\omega}^{\rho} \partial \bar{Y}_{\omega}^{\rho}} \frac{\partial \bar{Y}_{\omega}^{\rho}}{\partial \hat{Y}_{\alpha}^{\beta}} \frac{\partial \bar{Y}_{\omega}^{\rho}}{\partial \hat{Y}_{\gamma}^{\xi}}+\frac{\partial^{2} \bar{w}}{\partial \bar{Y}_{\omega}^{\rho} \partial \bar{Y}_{\pi}^{\eta}} \frac{\partial \bar{Y}_{\omega}^{\rho}}{\partial \hat{Y}_{\alpha}^{\beta}} \frac{\partial \bar{Y}_{\pi}^{\eta}}{\partial \hat{Y}_{\gamma}^{\xi}}+ \\
& \frac{\partial^{2} \bar{w}}{\partial \bar{Y}_{\pi}^{\eta} \partial \bar{Y}_{\omega}^{\rho}} \frac{\partial \bar{Y}_{\pi}^{\eta}}{\partial \hat{Y}_{\alpha}^{\beta}} \frac{\partial \bar{Y}_{\omega}^{\rho}}{\partial \hat{Y}_{\gamma}^{\xi}}+\frac{\partial^{2} \bar{w}}{\partial \bar{Y}_{\pi}^{\eta} \partial \bar{Y}_{\pi}^{\eta}} \frac{\partial \bar{Y}_{\pi}^{\eta}}{\partial \hat{Y}_{\alpha}^{\beta}} \frac{\partial \bar{Y}_{\pi}^{\eta}}{\partial \hat{Y}_{\gamma}^{\xi}} .
\end{aligned}
$$

sendo $\frac{\partial^{2} \bar{w}}{\partial \bar{Y}_{\omega}^{\rho} \partial \bar{Y}_{\pi}^{\eta}}$ a matriz Hessiana local da fibra dada na Eq. (4.21) para a fibra reta e na Eq. (4.39) para a fibra curva.

$\mathrm{Na}$ Eq. (4.53) não se considera soma para índices repetidos.

\subsection{PROCEDIMENTO MATRICIAL PARA O ACOPLAMENTO FIBRA-MATRIZ}

O acoplamento descrito na Seção 4.5 é válido para qualquer arranjo fibra-matriz mostrado na Figura 11.

Uma vez que as fibras são geradas sem a necessidade de coincidência com os nós da matriz, ou seja, os nós de uma fibra podem estar em quaisquer elementos da matriz, o procedimento para a introdução das fibras é generalizado nesta seção.

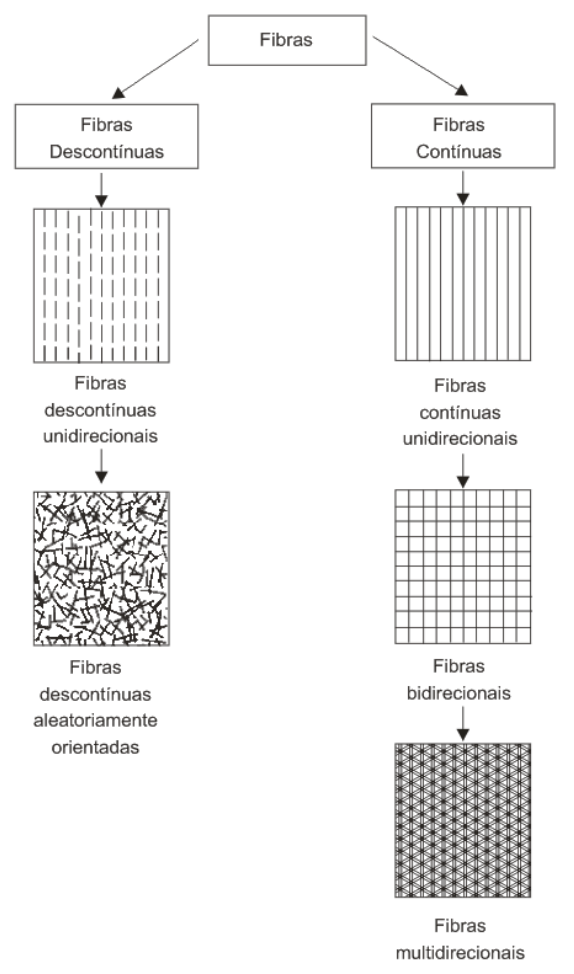

Figura 11 - Arranjos das fibras na matriz. Fonte: (DANIEL; ISHAI, 2006). 


\subsubsection{ACOPLAMENTO FIBRA RETA-MATRIZ}

Dependendo do arranjo fibra-matriz considerado (Figura 11) e da discretização adotada, as fibras, curtas ou longas, podem apresentar uma das configurações representadas na Figura 12: os dois nós da fibra podem estar inseridos no mesmo elemento finito de chapa (fibra 1); os nós da fibra podem estar em elementos adjacentes com $(\mathrm{GP}+1)$ nós em comum (fibra 2); os nós da fibra podem estar em elementos adjacentes com um nó em comum (fibra 3), ou ainda, cada um dos nós pode estar em elementos finitos não adjacentes, ou seja, sem qualquer nó em comum (fibra 4). Este fato determina os diversos valores que os termos do tipo $\frac{\partial \bar{Y}_{\pi}^{\eta}}{\partial \hat{Y}_{\gamma}^{\xi}}$ podem assumir na Eq. (4.53).

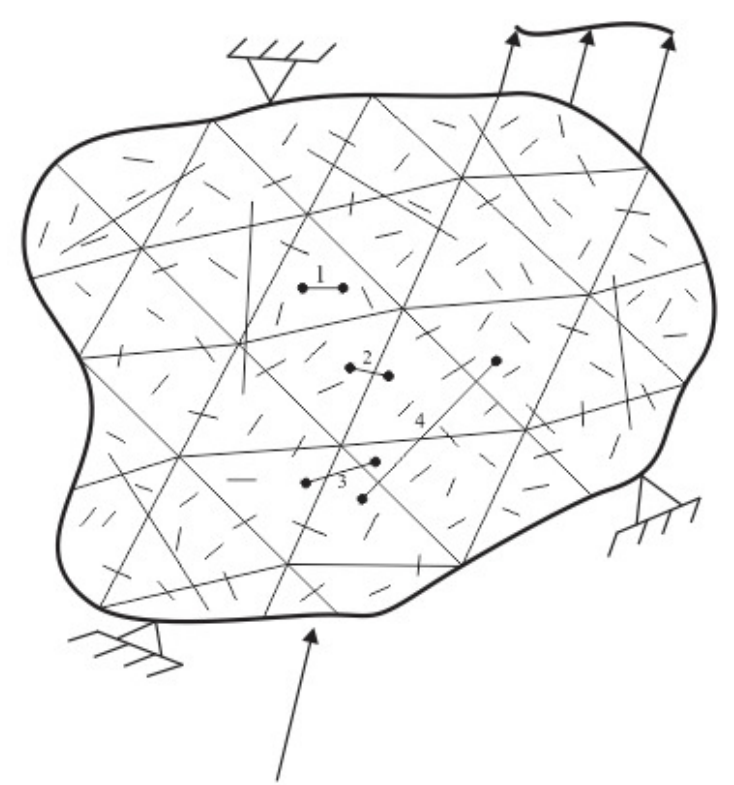

Figura 12 - Arranjo das fibras no domínio bidimensional.

Para simplificar o procedimento numérico, organiza-se de forma matricial a operação da Eq. (4.53) expandindo-se a matriz Hessiana local da fibra reta $\left[\overline{\mathrm{H}}^{\mathrm{f}}\right]_{4 \times 4}$ em uma matriz de ordem $(4 \hat{\mathrm{N}} \times 4 \hat{\mathrm{N}})$, onde $\hat{\mathrm{N}}$ é o número de nós dos elementos de chapa, ou seja,

$$
\left[\overline{\mathrm{H}}_{\mathrm{f}}\right]_{4 \hat{\mathrm{N}} \times 4 \hat{\mathrm{N}}}=\left[\bar{\phi}^{\beta}\right]_{4 \hat{\mathrm{N} \times 4}}^{\mathrm{T}} \cdot\left[\overline{\mathrm{H}}^{\mathrm{f}}\right]_{4 \times 4} \cdot\left[\bar{\phi}^{\beta}\right]_{4 \times 4 \hat{\mathrm{N}}},
$$


sendo $\left[\overline{\mathrm{H}}^{\mathrm{f}}\right]_{4 \times 4}$ obtida da Eq. (4.21) e $\hat{\mathrm{N}}$ obtido da Eq. (4.5). O subscrito $\mathrm{f}$ em $\left[\overline{\mathrm{H}}_{\mathrm{f}}\right]$ e o sobrescrito $f$ em $\left[\bar{H}_{f}\right]$ na Eq. (4.54) fazem distinção entre a matriz Hessiana expandida da fibra reta a ser contribuída na matriz Hessiana global do problema e a matriz Hessiana local da fibra reta, respectivamente. Observa-se da Eq. (4.53) que as funções de forma, para a fibra reta, são distribuídas em quatro quadrantes, sendo dois quadrantes preenchidos com os valores das funções de forma e dois quadrantes preenchidos com zeros, ou seja,

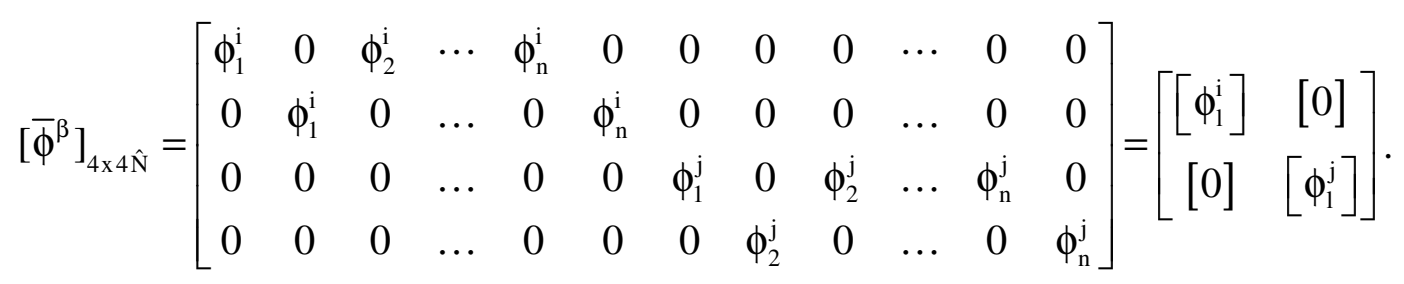

Na Eq. (4.55) o índice $i$ está relacionado ao nó inicial da fibra e o índice $j$ ao nó final. Após efetuar a operação matricial indicada na Eq. (4.54) tem-se uma matriz de rigidez para cada fibra contida no domínio que deve contribuir, respeitando a incidência dos elementos de chapa, na matriz de rigidez global do problema, evitando a soma direta ao elemento de chapa, pois existem termos adicionais que não são contemplados por estes, ou seja, a matriz de fibra expandida é menos esparsa do que duas matrizes de chapa, quando os nós do elemento de fibra estão contidos em mais de um elemento de chapa.

\subsubsection{ACOPLAMENTO FIBRA CURVA-MATRIZ}

O procedimento matricial para o acoplamento fibra curva-matriz é análogo ao procedimento apresentado para a fibra reta, ou seja, uma vez que o elemento de fibra curvo pode apresentar ordem qualquer de aproximação, a matriz de rigidez do mesmo é expandida em uma matriz de ordem $2\left(G^{f}+1\right) \hat{N} x 2\left(G P^{f}+1\right) \hat{N}$ e a Eq. (4.54) passa a ser expressa por:

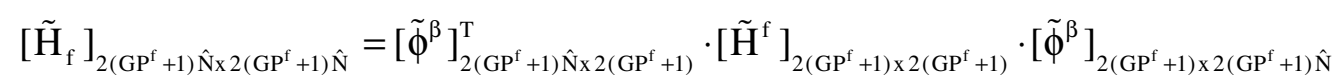

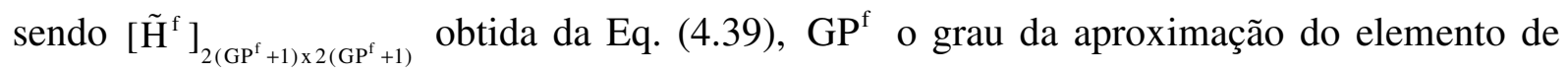
fibra curvo e $\hat{\mathrm{N}}$ obtido da Eq. (4.5). O subscrito f em $\left[\tilde{\mathrm{H}}_{\mathrm{f}}\right]$ e o sobrescrito f em $\left[\tilde{\mathrm{H}}^{\mathrm{f}}\right]$ na Eq. 
(4.56) fazem distinção entre a matriz Hessiana expandida da fibra curva a ser contribuída na matriz Hessiana global do problema e a matriz Hessiana local da fibra curva, respectivamente.

A Figura 13 mostra a sub-rotina principal para determinação da contribuição das fibras curvas de ordem qualquer para a energia total do sistema.

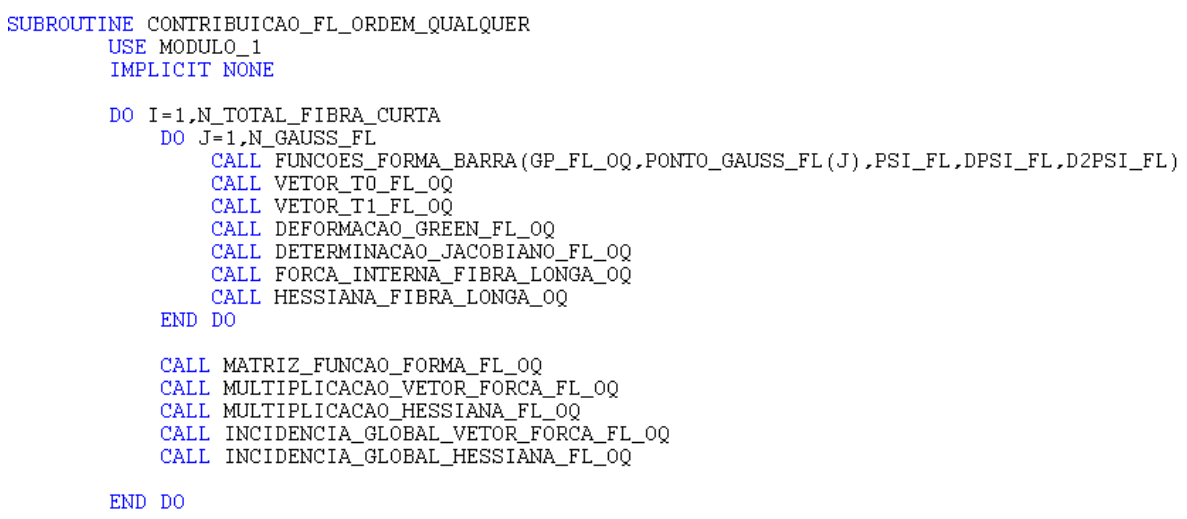

END SUBROUTINE CONTRIBUICAO_FL_ORDEM_QUALQUER

Figura 13 - Sub-rotina principal para contribuição das fibras na energia do sistema.

\subsection{CONECTIVIDADE NÓ DE FIBRA AO ELEMENTO BIDIMENSIONAL}

Como a malha das fibras é gerada independentemente da malha da matriz, ou seja, sem a necessidade de coincidência dos nós entre os dois meios, para proceder à contribuição das fibras no sistema é necessário conhecer a que elemento de chapa pertence o nó de fibra considerado. Para o elemento finito bidimensional curvo, a determinação da localização do ponto ocorre pela resolução de um sistema não linear de equações pelo método de NewtonRaphson e o procedimento é descrito a seguir.

Para iniciar a análise de domínios curvos reforçados com fibras curvas é necessário conhecer as coordenadas adimensionais dos nós de fibra em termos das coordenadas dos nós da matriz. Isto é possível pela resolução de um par de variáveis adimensionais $\left(\xi_{1}^{p}, \xi_{2}^{p}\right)$ do domínio bidimensional associadas com a posição física do nó de fibra pelo sistema não linear:

$$
\tilde{X}_{i}^{p}=\hat{\phi}_{l}\left(\xi_{1}^{p}, \xi_{2}^{p}\right) \hat{X}_{i}^{l}
$$

sendo $\hat{\phi}_{l}$ as funções de forma do elemento bidimensional, $\tilde{X}_{i}^{p}$ as coordenadas físicas do nós da fibra e $\hat{X}_{i}^{l}$ as coordenadas dos nós do elemento bidimensional. A Eq. (4.57) é expandida 
em série de Taylor, para um par de coordenadas adimensionais tentativa $\left(\xi_{1}^{p t}, \xi_{2}^{p t}\right)$, como:

$$
\tilde{X}_{i}^{p} \cong \hat{\phi}_{l}\left(\xi_{1}^{p t}, \xi_{2}^{p t}\right) \hat{X}_{i}^{l}+\left.\frac{\partial \hat{\phi}_{l}\left(\xi_{1}, \xi_{2}\right)}{\partial \xi_{j}}\right|_{\left(\xi_{1}^{p t}, \xi_{2}^{p t}\right)} \Delta \xi_{j} \text { ou } \tilde{X}_{i}^{p}=\tilde{X}_{i}^{p t}+H_{i j} \Delta \xi_{j}
$$

sendo $\tilde{X}_{i}^{p t}$ a posição tentativa dos nós da fibra calculada a partir da geometria do elemento bidimensional e das coordenadas adimensionais tentativa e $H_{i j}$ é uma matriz bidimensional. A correção das coordenadas adimensionais tentativa $\Delta \xi_{i}$ é calculada resolvendo-se o sistema não linear de equações:

$$
H_{i j} \Delta \xi_{j}=\tilde{X}_{i}^{p}-\tilde{X}_{i}^{p t}
$$

Este procedimento, método de Newton-Raphson, relaciona todos os nós de fibra ao correspondente elemento bidimensional, retornando o par de variáveis adimensionais $\left(\xi_{1}^{p}, \xi_{2}^{p}\right)$. A partir desta informação é possível conhecer as posições atuais dos nós de fibra em função das posições dos nós do elemento bidimensional, pela expressão:

$$
\tilde{Y}_{i}^{p}=\hat{\phi}_{l}\left(\xi_{1}^{p}, \xi_{2}^{p}\right) \hat{Y}_{i}^{l}
$$

sendo $\hat{Y}_{i}^{l}$ as posições atuais dos nós do elemento bidimensional. A Eq. (4.60) assegura a conexão dos nós de fibra à matriz considerada.

A Eq. (4.60) deve ser diferenciada em relação aos parâmetros nodais do elemento bidimensional, ou seja:

$$
\frac{\partial \tilde{Y}_{i}^{p}}{\partial \hat{Y}_{\alpha}^{\beta}}=\frac{\partial \hat{Y}_{i}^{l}}{\partial \hat{Y}_{\alpha}^{\beta}} \hat{\phi}_{l}\left(\xi_{l}^{p}, \xi_{2}^{p}\right)=\delta_{\alpha i} \delta_{\beta l} \hat{\phi}_{l}\left(\xi_{l}^{p}, \xi_{2}^{p}\right)=\delta_{\alpha i} \hat{\phi}_{\beta}\left(\xi_{l}^{p}, \xi_{2}^{p}\right) .
$$

Quando o nó da fibra pertence ao elemento bidimensional, a Eq. (4.61) indica que $\partial \tilde{Y}_{i}^{p} / \partial \hat{Y}_{\alpha}^{\beta}=\hat{\phi}_{\beta}\left(\xi_{1}^{p}, \xi_{2}^{p}\right)$ se a direção $\alpha$ do elemento bidimensional é igual à direção $i$ da fibra e zero caso contrário.

A ideia inicial do procedimento aqui descrito foi apresentada por Vanalli (2004) e Vanalli, Paccola e Coda (2008) para o caso de fibras retas e elementos triangulares bidimensionais em aplicações lineares. 
A Figura 14 mostra a estrutura do programa principal para a análise de domínios bidimensionais reforçados com fibras conforme implementado neste trabalho.

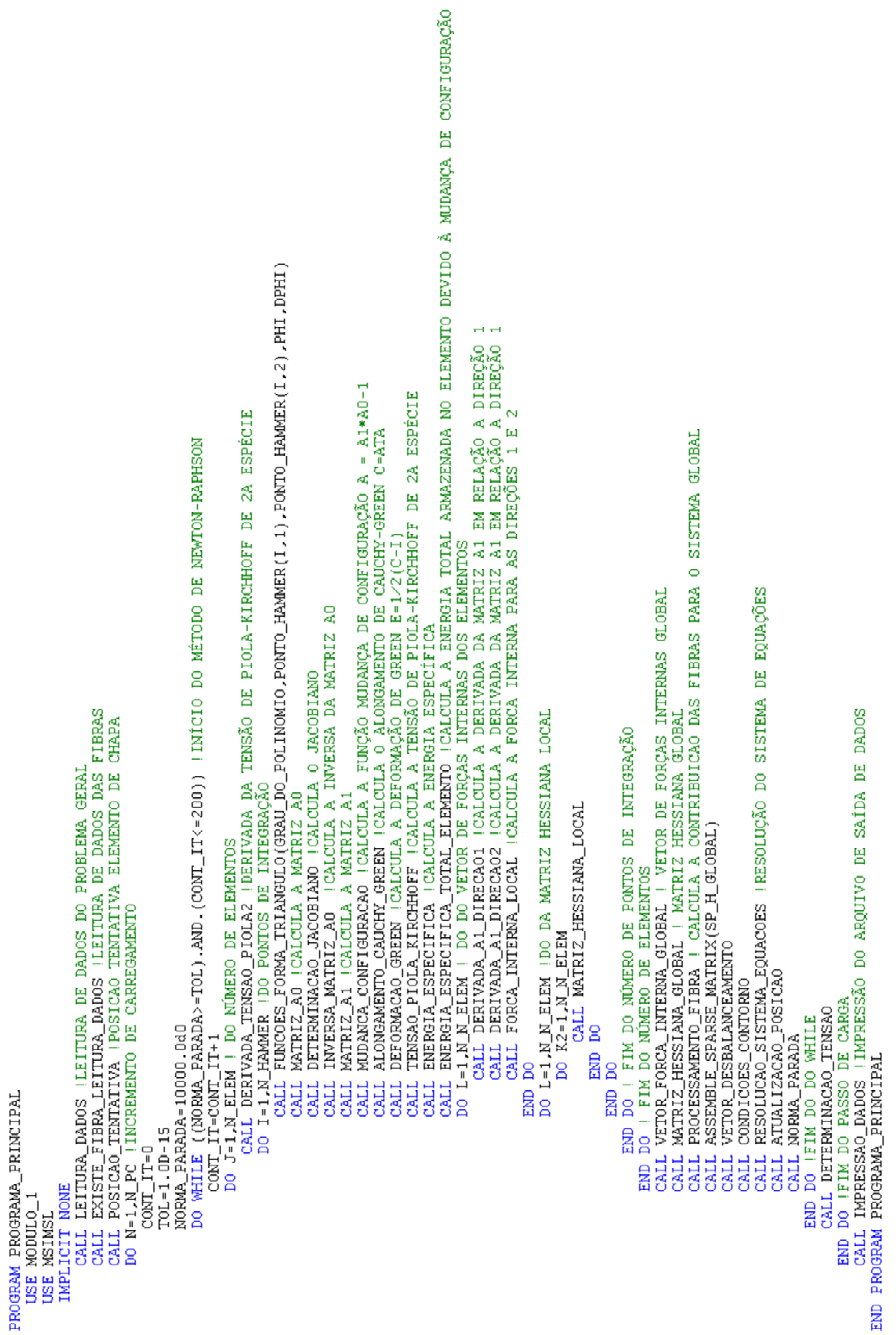

Figura 14 - Programa principal para a análise de domínios bidimensionais reforçados com fibras. 


\subsection{EXEMPLOS NUMÉRICOS}

Para validar a formulação introduzida e demonstrar as suas potencialidades cinco exemplos numéricos são apresentados nesta seção. O primeiro tem por objetivo verificar se o acoplamento fibra-matriz proposto funciona adequadamente para análises em grandes deslocamentos. O segundo tem por objetivo verificar a conformidade do acoplamento fibramatriz. O terceiro tem por objetivo demonstrar as potencialidades da formulação para a análise de domínios bidimensionais reforçados com fibras aleatórias. O quarto compara os resultados obtidos com a presente formulação com resultados obtidos na literatura e o quinto tem por objetivo analisar a convergência do campo de tensões desenvolvido pelo domínio bidimensional.

\subsubsection{EXEMPLO 1: VIGA REFORÇADA COM FIBRAS SUBMETIDA A GRANDES DESLOCAMENTOS}

Este exemplo foi utilizado para verificar se o acoplamento fibra-matriz está funcionando adequadamente para análises em grandes deslocamentos. As propriedades geométricas adotadas para a viga são: $L=300 \mathrm{~cm}, h=10 \mathrm{~cm}, \quad b=1 \mathrm{~cm}, d=2,5 \mathrm{~cm}$ e $h^{\prime}=5 \mathrm{~cm}$. O módulo de elasticidade e o coeficiente de Poisson da matriz são dados, respectivamente, por $E_{m}=21 G P a$ e $v_{m}=0,0$. Para as fibras, o módulo de elasticidade e a área da seção transversal são dados, respectivamente, por $E_{f}=210 G P a$ e $A_{f}=0,1 \mathrm{~cm}^{2}$.

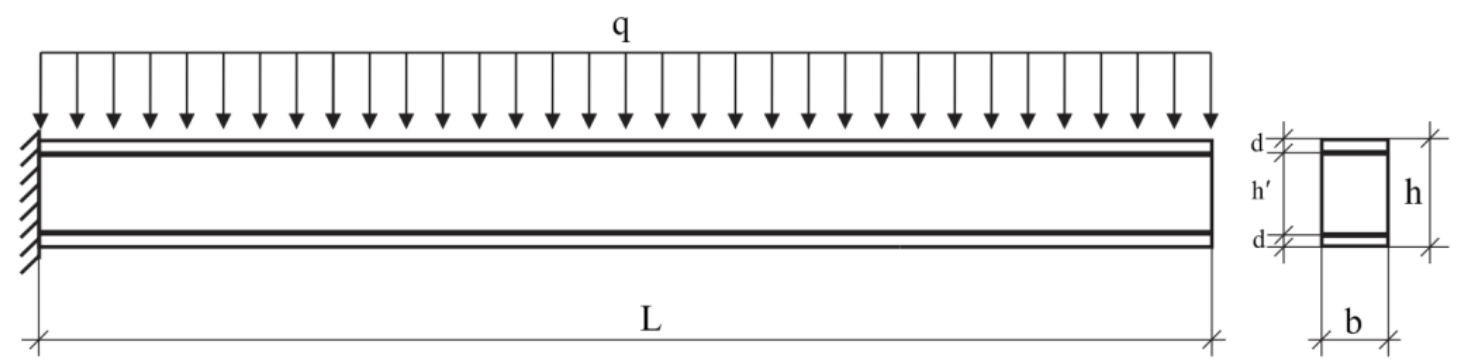

Figura 15 - Viga simplesmente engastada reforçada com fibras. 
O problema foi estudado considerando-se os regimes linear e não linear, para a viga com fibras e sem fibras. Para o caso linear o carregamento transversal aplicado é $q=0,5 \mathrm{~N} / \mathrm{cm}$ e para o caso não linear $q=50 \mathrm{~N} / \mathrm{cm}$. Os resultados em grandes deslocamentos são comparados com resultados de referência obtidos com uma formulação denominada barra geral 3D que utiliza cinemática de Timoshenko-Reissner apresentada por Coda (2009), Coda e Paccola (2010) e Coda e Paccola (2011). Para a análise em pequenos deslocamentos, além da formulação de barra geral 3D utilizam-se as expressões analíticas da Resistência dos Materiais.

Para a formulação proposta neste trabalho, a matriz foi discretizada em 300 elementos finitos bidimensionais quadráticos, totalizando 671 nós e 1342 graus de liberdade. Para as fibras foram adotadas duas discretizações. Uma com 120 elementos lineares e outra com 60 elementos quadráticos. Para a formulação de barra geral 3D foram utilizados 10 elementos com aproximação do quinto grau para as variáveis. A análise não linear foi realizada em 10 passos de carga. A Figura 16 e a Figura 17 mostram as configurações deformadas obtidas para a viga sem fibras e com fibras, respectivamente. Não foi observada qualquer diferença entre os resultados obtidos usando fibras lineares e quadráticas em todas as análises realizadas, validando a formulação para a análise de fibras curvas de ordem qualquer proposta.

$\mathrm{Na}$ análise linear sem fibras, a flecha máxima na extremidade livre da viga obtida com a formulação proposta foi de $\delta \cong 2,896 \mathrm{~cm}$, Figura 16a, e o resultado obtido da Resistência dos Materiais foi de $\delta \cong 2,892 \mathrm{~cm}$, resultando em um desvio entre as soluções de aproximadamente $0,138 \%$.

Para a análise linear considerando as fibras, a flecha máxima na extremidade livre da viga obtida com a formulação proposta foi de $\delta \cong 1,1606 \mathrm{~cm}$, Figura 17 a, e o resultado obtido da Resistência dos Materiais considerando a seção homogeneizada foi de $\delta \cong 1,1571 \mathrm{~cm}$, correspondendo a um desvio entre as soluções de aproximadamente $0,302 \%$.

Para a análise não linear sem fibras, a flecha máxima na extremidade livre da barra obtida com a formulação de barra geral 3D foi de $\delta \cong 189,269 \mathrm{~cm}$, Figura $16 \mathrm{~d}$, e a flecha máxima no nó central da extremidade livre da viga com a formulação proposta foi de $\delta \cong 193,053 \mathrm{~cm}$, Figura 16c, resultando em um desvio entre as soluções de aproximadamente 1,999\% . Acredita-se que os resultados obtidos são satisfatórios visto que a formulação de referência, barra geral 3D, utiliza cinemática de Timoshenko-Reissner em que seções planas permanecem planas após a deformação. Uma vez que a formulação apresentada neste trabalho utiliza elementos finitos de chapa espera-se uma estrutura mais flexível conforme resultados 
obtidos.
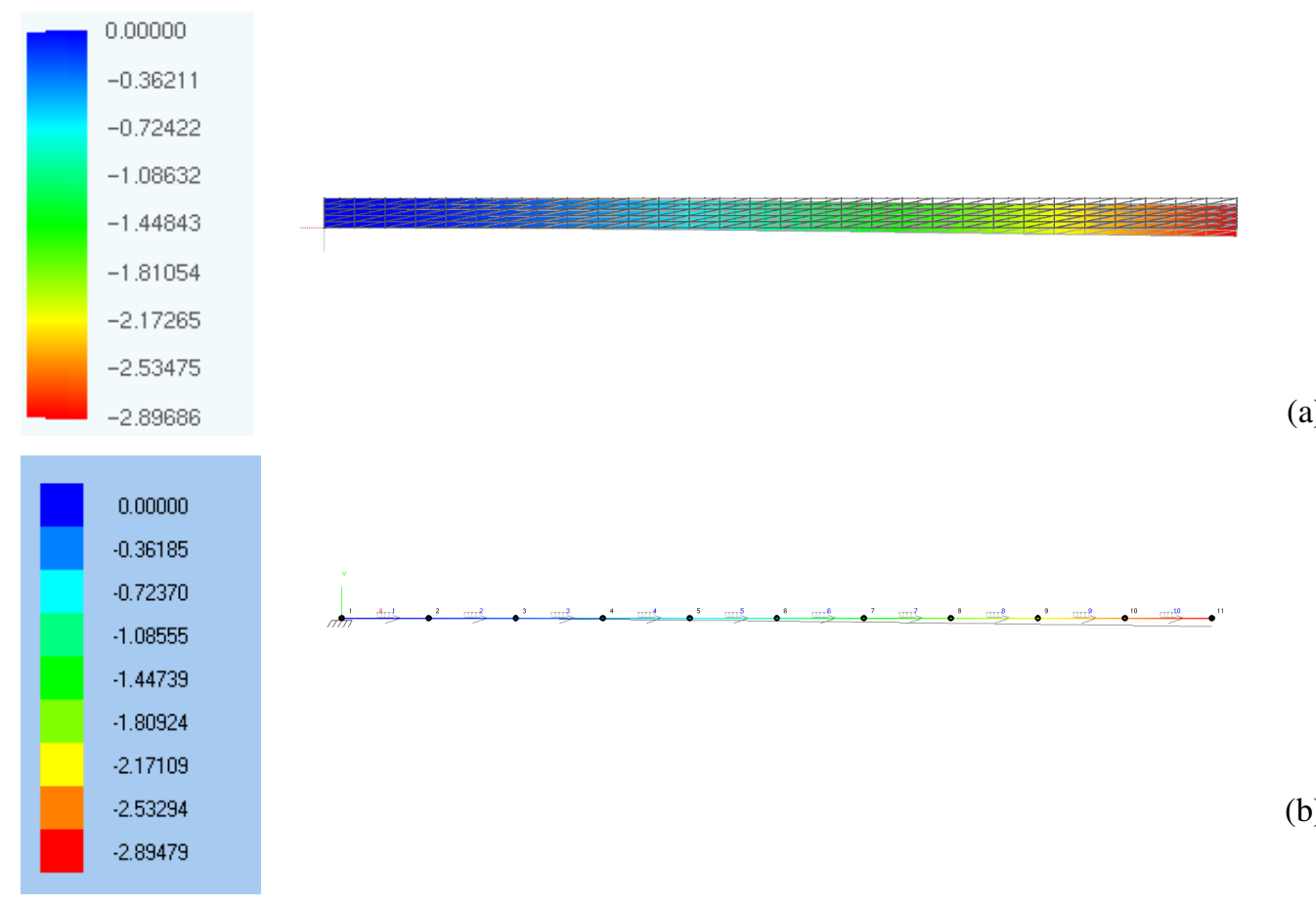

(a)

(b)
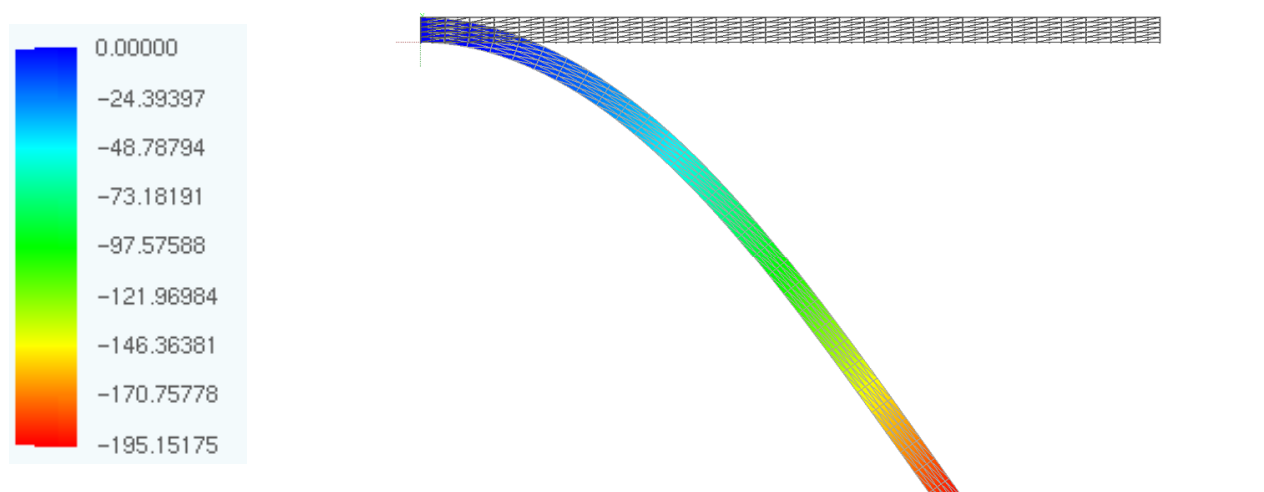

(c)

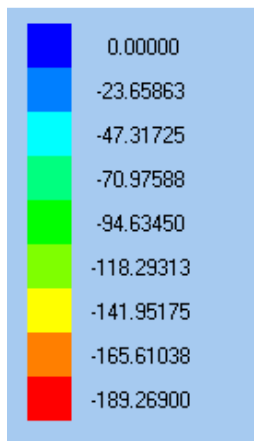

(d)

Figura 16 - Configuração deformada da viga homogênea em cm: (a) presente formulação - linear; (b) barra geral 3D - linear; (c) presente formulação - não linear; (d) barra geral 3D - não linear. 

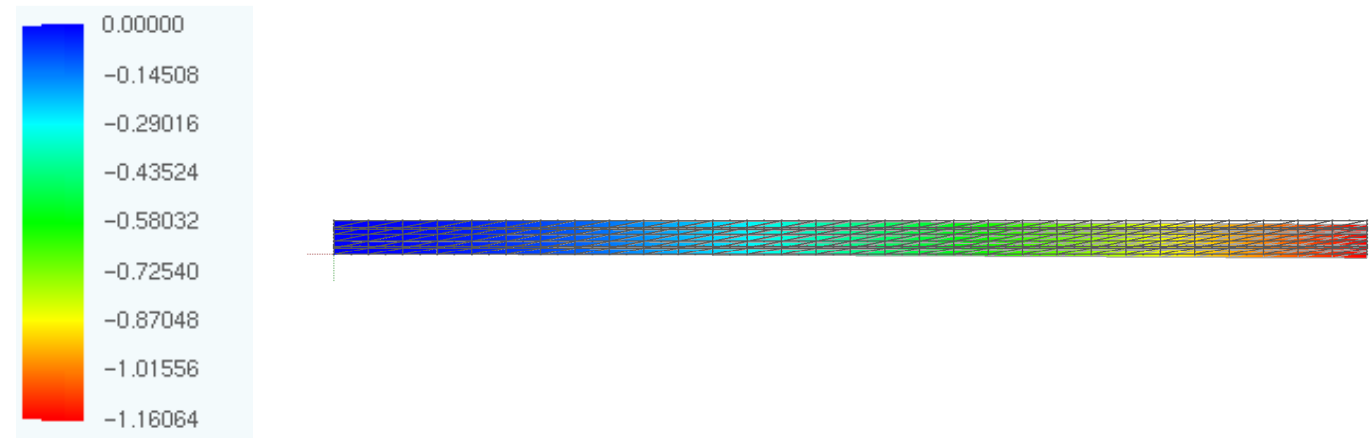

$-0.72540$

$-0.87048$

$-1.16064$

(a)
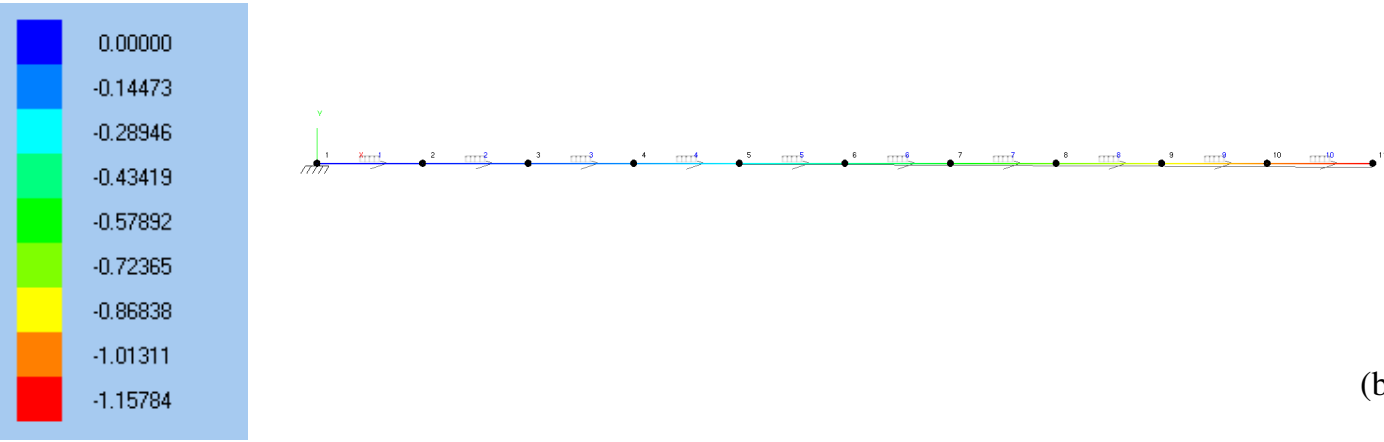

(b)
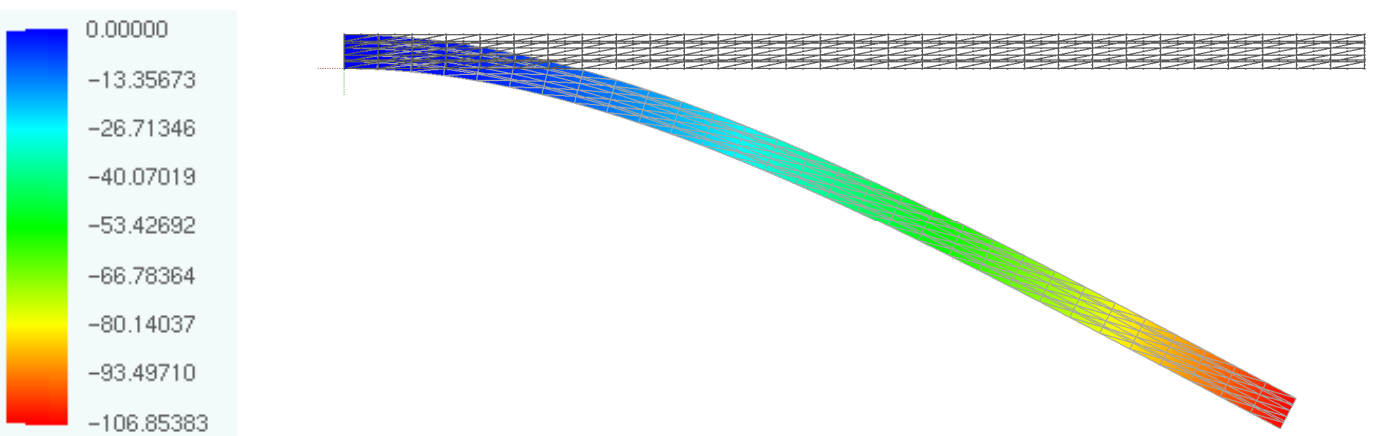

(c)
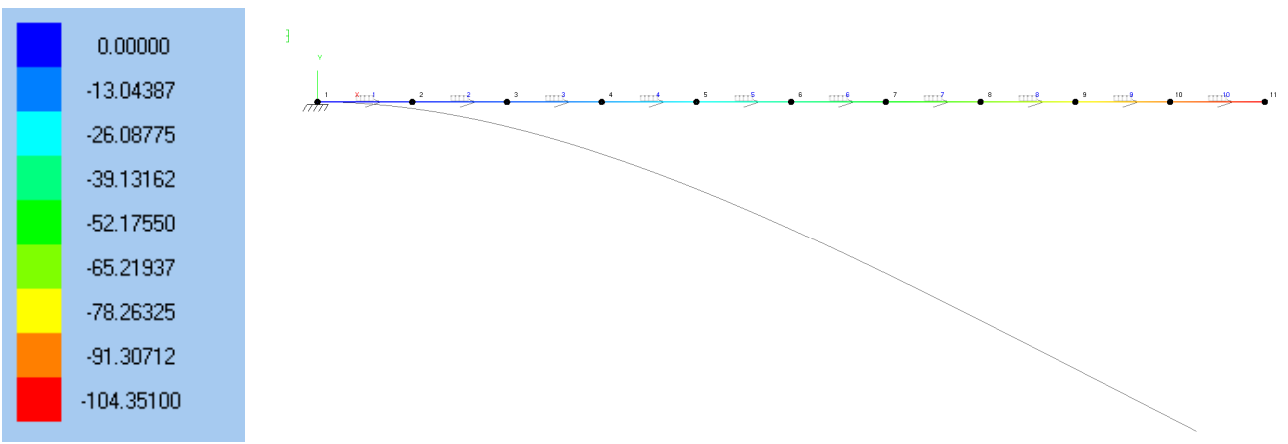

(d)

Figura 17 - Configuração deformada da viga com fibras: (a) presente formulação - linear; (b) barra geral 3D linear; (c) presente formulação - não linear; (d) barra geral 3D - não linear. 
Para a análise não linear com fibras, a flecha máxima no nó central da extremidade livre da viga obtida com a formulação proposta foi de $\delta \cong 106,393 \mathrm{~cm}$, Figura $17 \mathrm{c}$, e a flecha máxima na extremidade livre da barra obtida com a formulação de barra geral 3D foi de $\delta \cong 104,351 \mathrm{~cm}$, Figura $17 \mathrm{~d}$, resultando em um desvio entre as soluções de aproximadamente 1,957\% . Da mesma forma que no caso anterior, é possível observar que a estrutura se mostrou mais flexível com a formulação proposta do que com a formulação de barra geral 3D, ou seja, o modelo de chapa confere mais liberdade ao contínuo modelado. Entretanto, os resultados são bastante próximos e validam o reforço em elementos finitos de fibras curvos implementado.

Os resultados discutidos anteriormente estão organizados na Tabela 1.

Tabela 1 - Deslocamentos máximos na extremidade livre da viga para os diferentes casos analisados em $(\mathrm{cm})$.

\begin{tabular}{|c|c|c|c|c|c|}
\hline \multirow{3}{*}{ DISCRETIZAÇÃO } & \multicolumn{5}{|c|}{ TIPO DE ANÁLISE } \\
\hline & \multicolumn{3}{|c|}{ LINEAR } & \multicolumn{2}{|c|}{ NÃO LINEAR } \\
\hline & PRESENTE & $\begin{array}{c}\text { RESISTÊNCIA } \\
\text { DOS MATERIAIS }\end{array}$ & BARRA GERAL 3D & PRESENTE & BARRA GERAL 3D \\
\hline sem fibras & 2,896 & 2,892 & 2,894 & 193,053 & 189,269 \\
\hline DESVIO (\%) & \multicolumn{2}{|c|}{$\mathbf{0 , 1 3 8}$} & 0,069 & \multicolumn{2}{|c|}{1,999} \\
\hline com fibras & 1,1606 & 1,1571 & 1,1578 & 106,393 & 104,351 \\
\hline DESVIO $(\%)$ & \multicolumn{2}{|c|}{0,302} & 0,242 & \multicolumn{2}{|c|}{1,957} \\
\hline
\end{tabular}

A Figura 18 compara as curvas de força normal na fibra superior obtidas com a formulação numérica e com a expressão analítica para pequenos deslocamentos dada por:

$$
F_{n}= \pm \frac{3 q h^{\prime} E_{f} A_{f}}{E_{m} b h^{3}+6 E_{f} A_{f} h^{2}}(x-L)^{2},
$$

onde o sinal positivo está relacionado à fibra superior e o sinal negativo à fibra inferior.

Para esta análise considerou-se $q=0,5 \mathrm{~N} / \mathrm{cm}$ e é possível observar da Figura 18 que os resultados obtidos estão em conformidade com aqueles esperados.

A solução numérica é bidimensional e a solução analítica é baseada na solução técnica de engenharia. Assim, as forças de tração e de compressão são diferentes na solução numérica devido à influência das forças de cisalhamento. No entanto, o valor médio é muito próximo da 
solução analítica.

A Tabela 2 compara os resultados obtidos com as formulações numérica e analítica em três pontos ao longo da fibra.

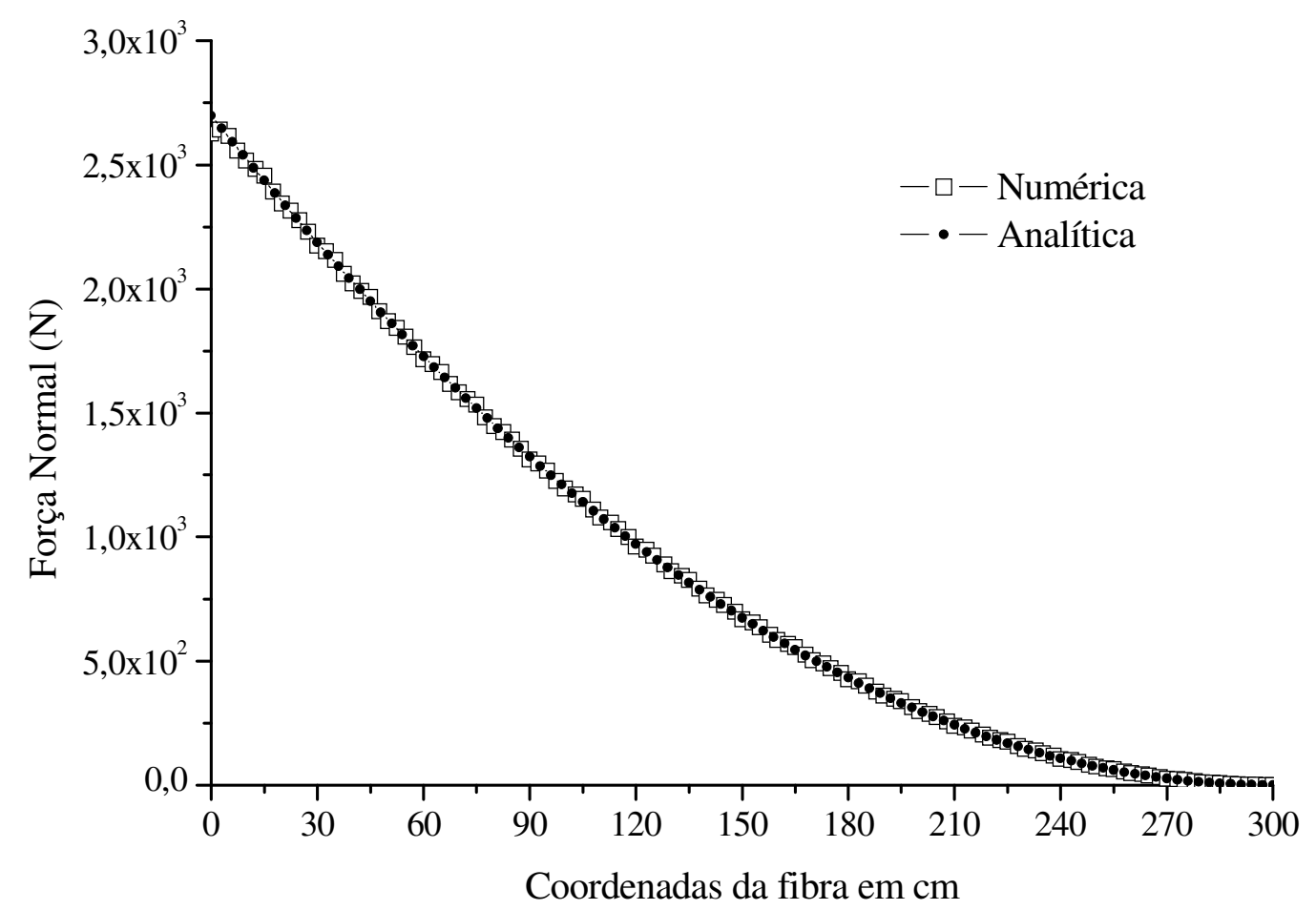

Figura 18 - Força normal na fibra superior.

Tabela 2 - Valores analíticos e numéricos de força normal para pontos selecionados em (N).

\begin{tabular}{c|c|c|c|c|c}
\hline $\begin{array}{c}\text { Posição horizontal } \\
(\mathrm{X} \mathrm{em} \mathrm{cm})\end{array}$ & Analítica & $\begin{array}{c}\text { Numérica } \\
\text { Superior }\end{array}$ & $\begin{array}{c}\text { Numérica } \\
\text { Inferior }\end{array}$ & $\begin{array}{c}\text { Numérica } \\
\text { Média }\end{array}$ & $\begin{array}{c}\text { Diferença } \\
\text { Relativa }\end{array}$ \\
\hline 10 & 2523 & 2540,78 & $-2511,33$ & 2526,06 & $0,12 \%$ \\
\hline 150 & 675 & 682,72 & $-667,94$ & 675,33 & $0,05 \%$ \\
\hline 290 & 3 & 3,42 & $-2,66$ & 3,04 & $1,33 \%$ \\
\hline
\end{tabular}

\subsubsection{EXEMPLO 2: CONFORMIDADE DO MAPEAMENTO FIBRA-MATRIZ}

Neste exemplo, utilizou-se a análise de um domínio bidimensional hipotético para demonstrar a aderência total entre as fibras e a matriz quando a ordem de aproximação das 
fibras é maior ou igual a ordem de aproximação da matriz.

Esta análise foi realizada em um único elemento bidimensional reforçado por uma fibra. Para modelar o elemento bidimensional foram utilizadas quatro aproximações, linear, quadrática, cúbica e do quarto grau. Em cada uma destas aproximações variou-se a ordem de aproximação da fibra de linear até a quarta ordem.

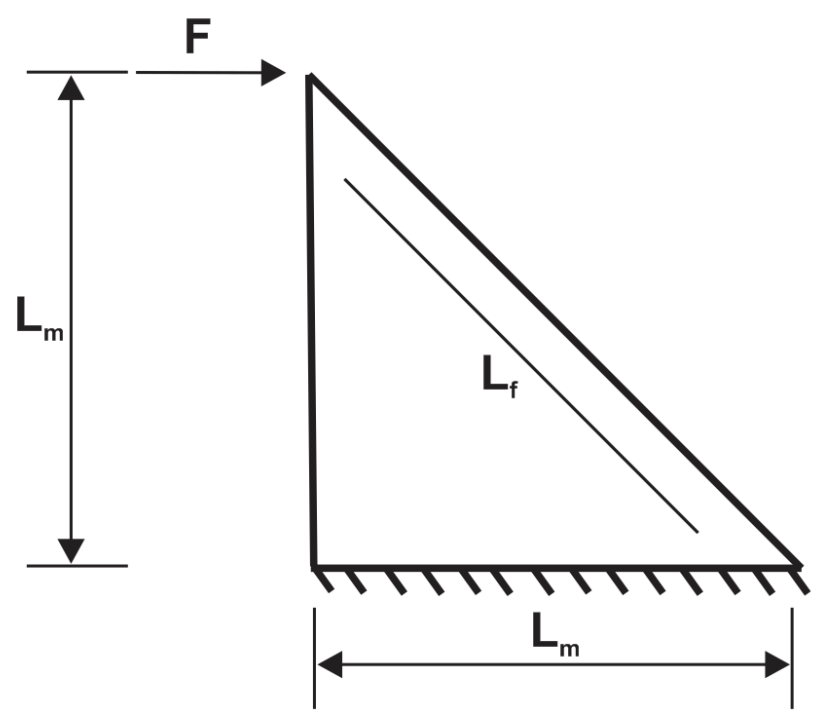

Figura 19 - Domínio bidimensional hipotético reforçado com fibra.

O módulo de elasticidade e o coeficiente de Poisson da matriz são dados, respectivamente, por $E_{m}=10^{4} \mathrm{~N} / \mathrm{m}^{2}$ e $v_{m}=0,0$ enquanto que o módulo de elasticidade e a área da seção transversal da fibra são dados, respectivamente, por $E_{f}=10^{5} \mathrm{~N} / \mathrm{m}^{2}$ e $A_{f}=0,1 \mathrm{~cm}^{2}$. Os pontos inferiores do domínio bidimensional estão totalmente restritos e uma força horizontal de $F=5,0 \mathrm{~N}$ é aplicada no nó de topo conforme mostrado na Figura 19. Os lados do domínio bidimensional têm o mesmo comprimento $L_{m}=200 \mathrm{~cm}$ e o comprimento da fibra é $L_{f}=180 \mathrm{~cm}$ localizada a $10 \mathrm{~cm}$ dos vértices do triângulo.

O objetivo desta análise foi verificar se os pontos ao longo da fibra, exceto os nós, apresentam movimento relativo quando comparados aos pontos correspondentes no contínuo após uma mudança na configuração. Para esta verificação foi necessário determinar as coordenadas adimensionais dos pontos de interesse na fibra. Neste caso, foram selecionados doze pontos igualmente espaçados ao longo da fibra. Para determinar as coordenadas físicas destes pontos na configuração inicial foram utilizadas as funções de forma da fibra. Com estas 
coordenadas físicas, determinam-se as correspondentes coordenadas adimensionais no elemento bidimensional, Eqs. (4.57)-(4.59). Depois de aplicar a força externa e determinar as posições nodais de equilíbrio, utilizam-se as funções de forma da fibra para determinar as posições atuais dos pontos escolhidos e as funções de forma do elemento bidimensional para determinar as correspondentes posições atuais do contínuo. Quando os valores obtidos são diferentes entre si ocorre movimento relativo entre o meio contínuo e a fibra, caso contrário a condição de aderência total é assegurada.

A Figura 20 e a Tabela 3 mostram os resultados obtidos para o caso de matriz com aproximação cúbica e fibra com aproximação variando de linear ao quarto grau. Os resultados obtidos para as demais aproximações do domínio bidimensional, linear, quadrática e do quarto grau são apresentados no APÊNDICE B.

A primeira linha na Figura 20 mostra a discretização do domínio bidimensional e da fibra, enquanto que a segunda linha ilustra os deslocamentos horizontais e a terceira ilustra os deslocamentos verticais. Neste caso, a aproximação do domínio bidimensional é sempre cúbica e a aproximação das fibras varia de linear ao quarto grau da esquerda para a direita, respectivamente.
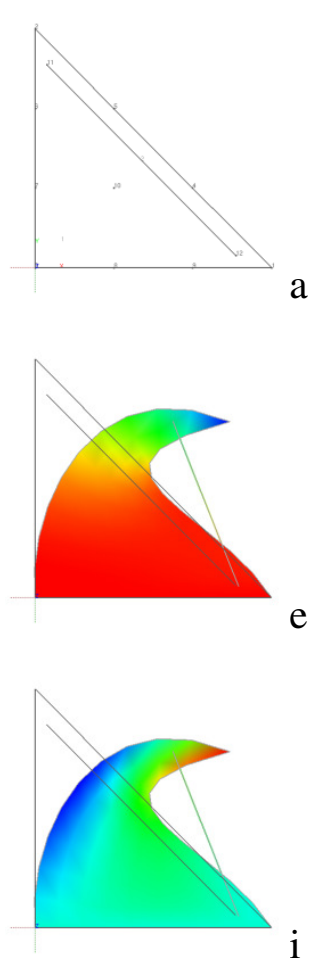
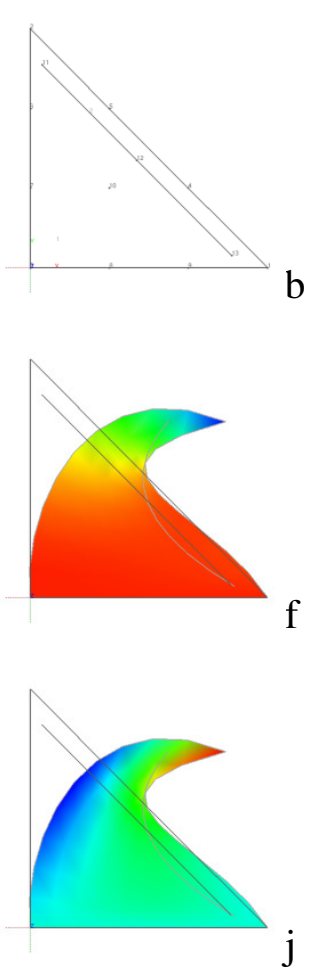
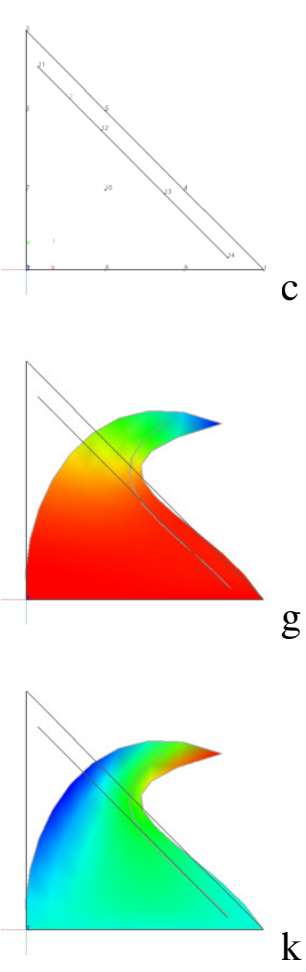
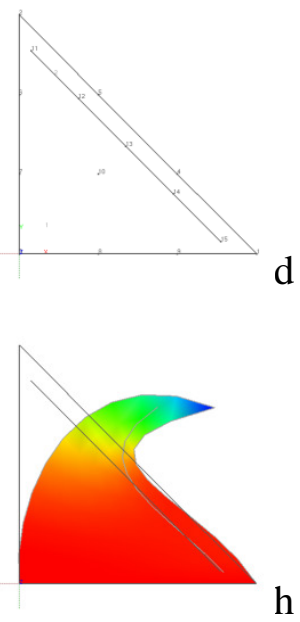

d

h

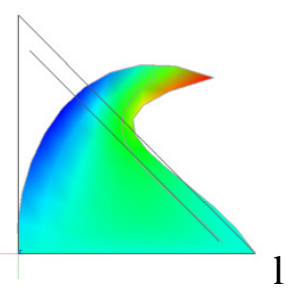

Figura 20 - Matriz com aproximação cúbica e fibras com aproximação variando de linear ao quarto grau. 
Tabela 3 - Matriz com aproximação cúbica e fibras com aproximação variando de linear ao quarto grau.

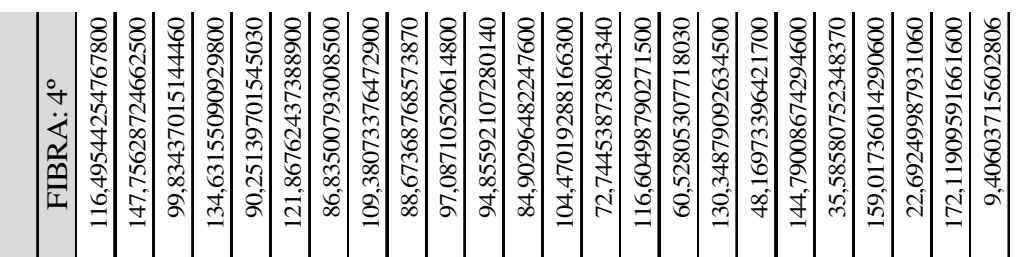

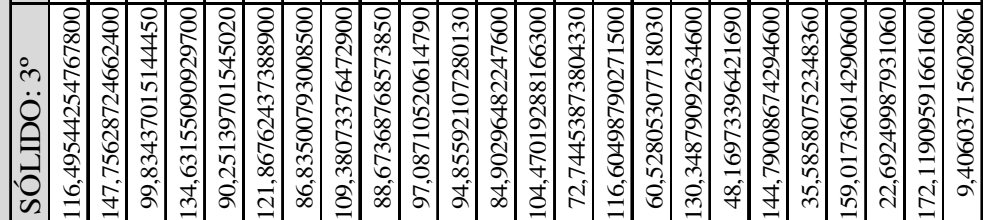
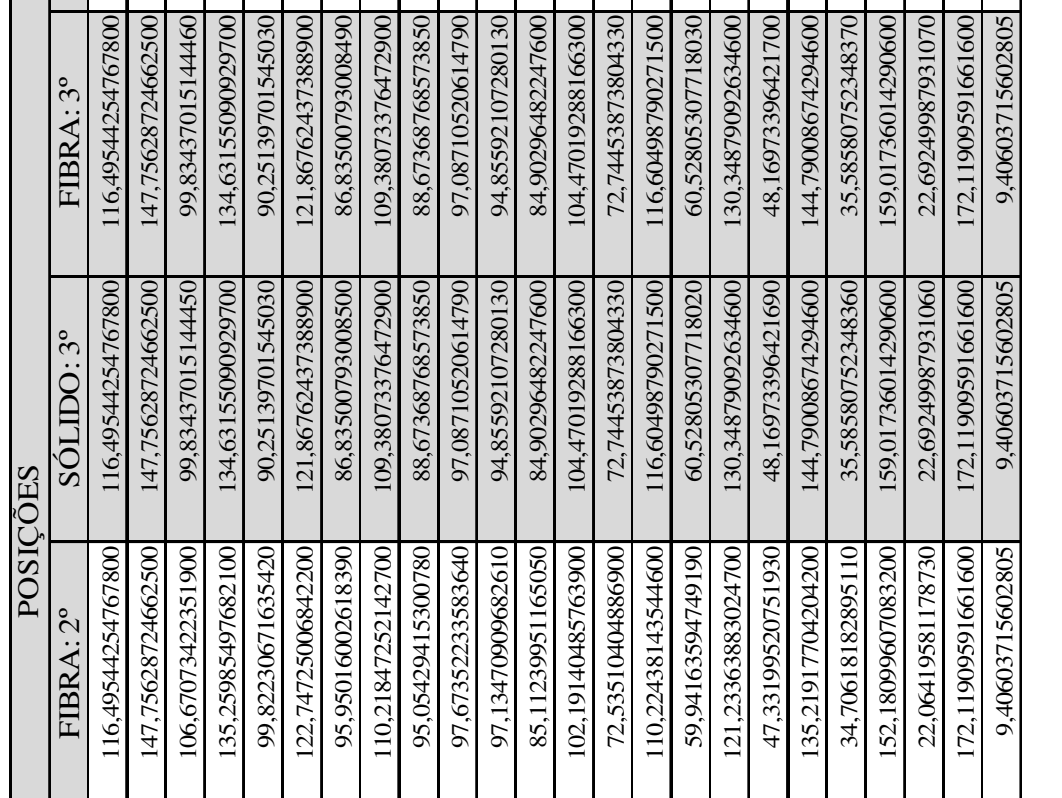

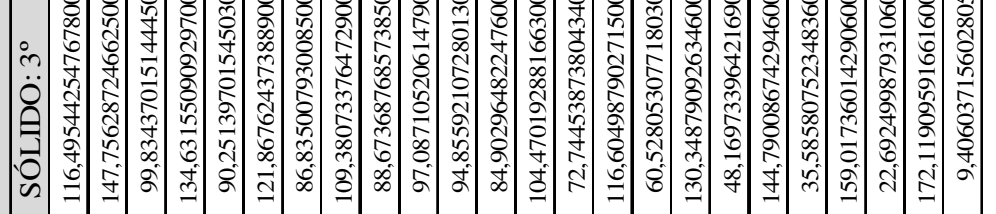

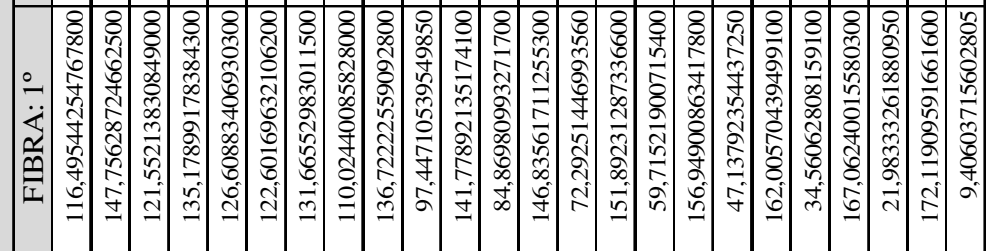

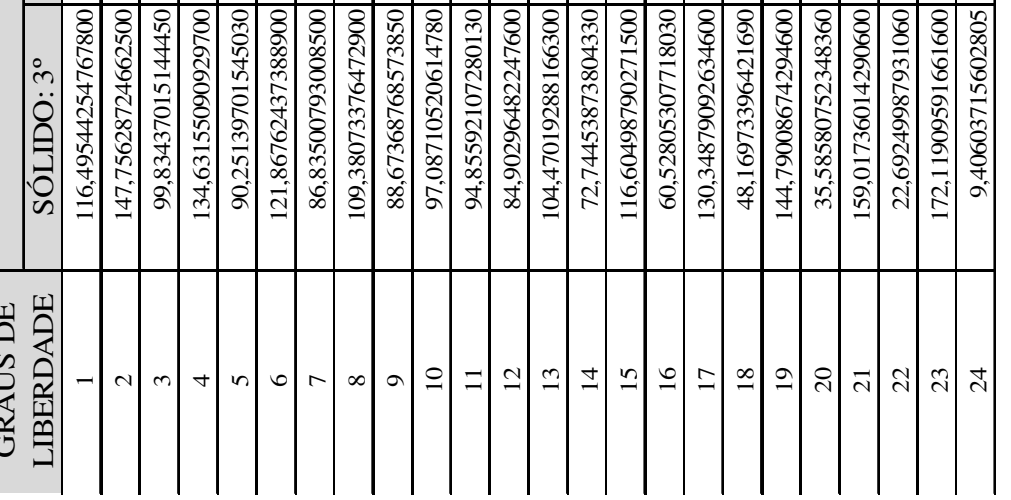


Da Figura 20 é possível observar que existe deslocamento relativo entre os pontos do contínuo e as aproximações linear e quadrática da fibra, mas não existe movimento para as aproximações do terceiro e quarto grau. Este comportamento foi verificado numericamente conforme mostrado na Tabela 3, onde as posições atuais da fibra são comparadas com os correspondentes pontos do contínuo.

Com base em todos os experimentos numéricos realizados para as diferentes discretizações adotadas para a matriz e para a fibra, observa-se a partir dos resultados obtidos que a condição de aderência total fibra-matriz é garantida se a ordem de aproximação da fibra é pelo menos a mesma ordem de aproximação adotada para o elemento bidimensional.

\subsubsection{EXEMPLO 3: CHAPA REFORÇADA COM FIBRAS ALEATÓRIAS}

Neste exemplo, estuda-se o comportamento dos deslocamentos horizontais e verticais de uma chapa enrijecida com fibras dispostas aleatoriamente no domínio. A chapa, engastada em duas extremidades é solicitada por um carregamento uniformemente distribuído conforme mostrado na Figura 21.

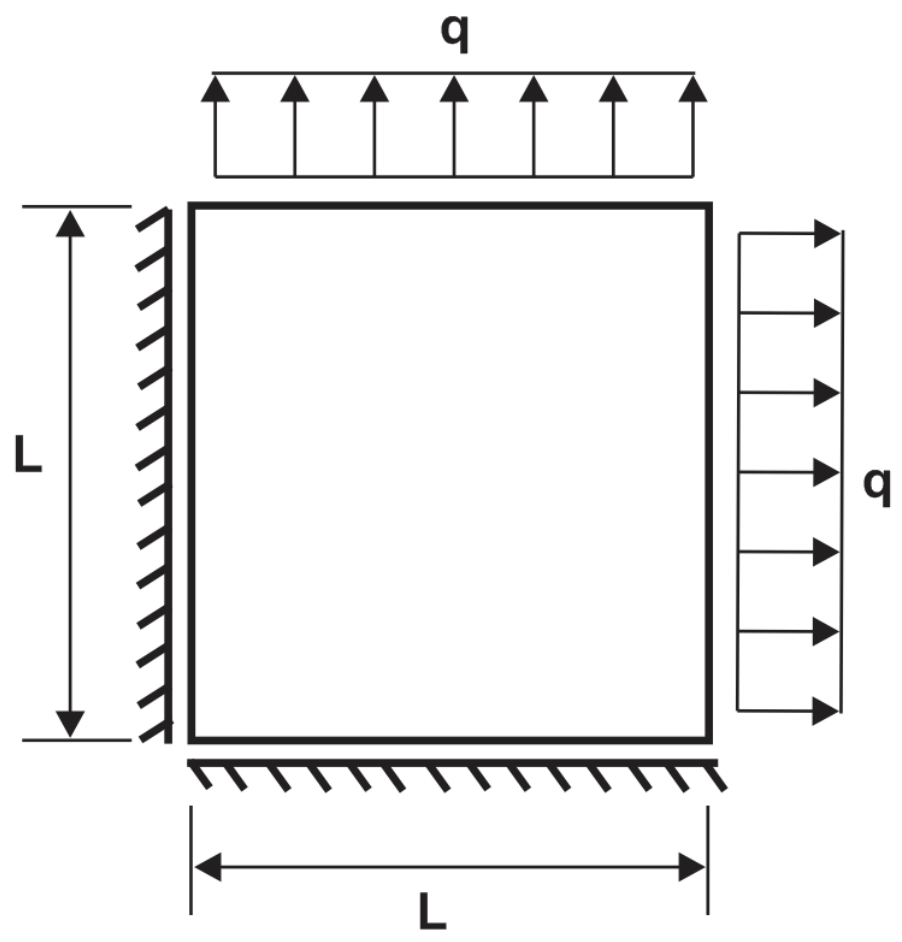

Figura 21 - Chapa engastada reforçada com fibras. 
O objetivo deste exemplo é verificar se o mapeamento das fibras aleatórias na análise de domínios bidimensionais está sendo feito corretamente e demonstrar potencialidades da formulação proposta.

A Figura 22 mostra os deslocamentos horizontais e verticais da chapa da Figura 21 considerando $L_{m}=200 \mathrm{~cm}, q=300 \mathrm{~N} / \mathrm{cm}, E_{m}=1 \mathrm{~N} / \mathrm{cm}^{2}$ e $v_{m}=0,0$.

Para analisar o mapeamento foram distribuídas aleatoriamente noventa fibras no interior da chapa. O módulo de elasticidade, a área da seção transversal e o comprimento das fibras são dados, respectivamente, por $E_{f}=10 \mathrm{~N} / \mathrm{cm}^{2}, A_{f}=0,1 \mathrm{~cm}^{2}$ e $L_{f}=20 \mathrm{~cm}$.

$\mathrm{Na}$ primeira análise, considerou-se que as fibras aleatórias introduzidas possuem módulo de elasticidade nulo, ou seja, não devem contribuir para a rigidez do sistema e a análise deve retornar os resultados mostrados na Figura 22 com a posição das fibras atualizadas conforme mostra a Figura 23.

Em seguida, repetiu-se a análise, considerando-se o módulo de elasticidade das fibras $E_{f}=10 \mathrm{~N} / \mathrm{cm}^{2}$. Os resultados obtidos para os deslocamentos horizontais e verticais podem ser visualizados na Figura 24. Observa-se que a consideração das fibras proporcionou um aumento de rigidez na direção horizontal de aproximadamente 0,673\% e de aproximadamente $1,542 \%$ na direção vertical. Nestas análises foram utilizados apenas dois elementos finitos na discretização da matriz.

A mesma análise foi realizada considerando-se uma discretização para a matriz com duzentos elementos finitos. Os resultados obtidos para os deslocamentos da chapa, para a atualização das posições das fibras e para os deslocamentos da chapa enrijecida podem ser visualizados na Figura 24, na Figura 25 e na Figura 26, respectivamente.

Para esta discretização observa-se um aumento de rigidez na direção horizontal de aproximadamente $0,615 \%$ e de aproximadamente $1,391 \%$ na direção vertical.

Os valores máximos obtidos em cada análise e os desvios observados para o caso em que o módulo de elasticidade da fibra é dado por $E_{f}=10 \mathrm{~N} / \mathrm{cm}^{2}$ estão organizados na Tabela 4.

Relembra-se que o objetivo deste exemplo é demonstrar o mapeamento das fibras aleatórias devido à formulação apresentada e que exemplos com proporções mais realistas entre fibras e matriz podem ser testados. 


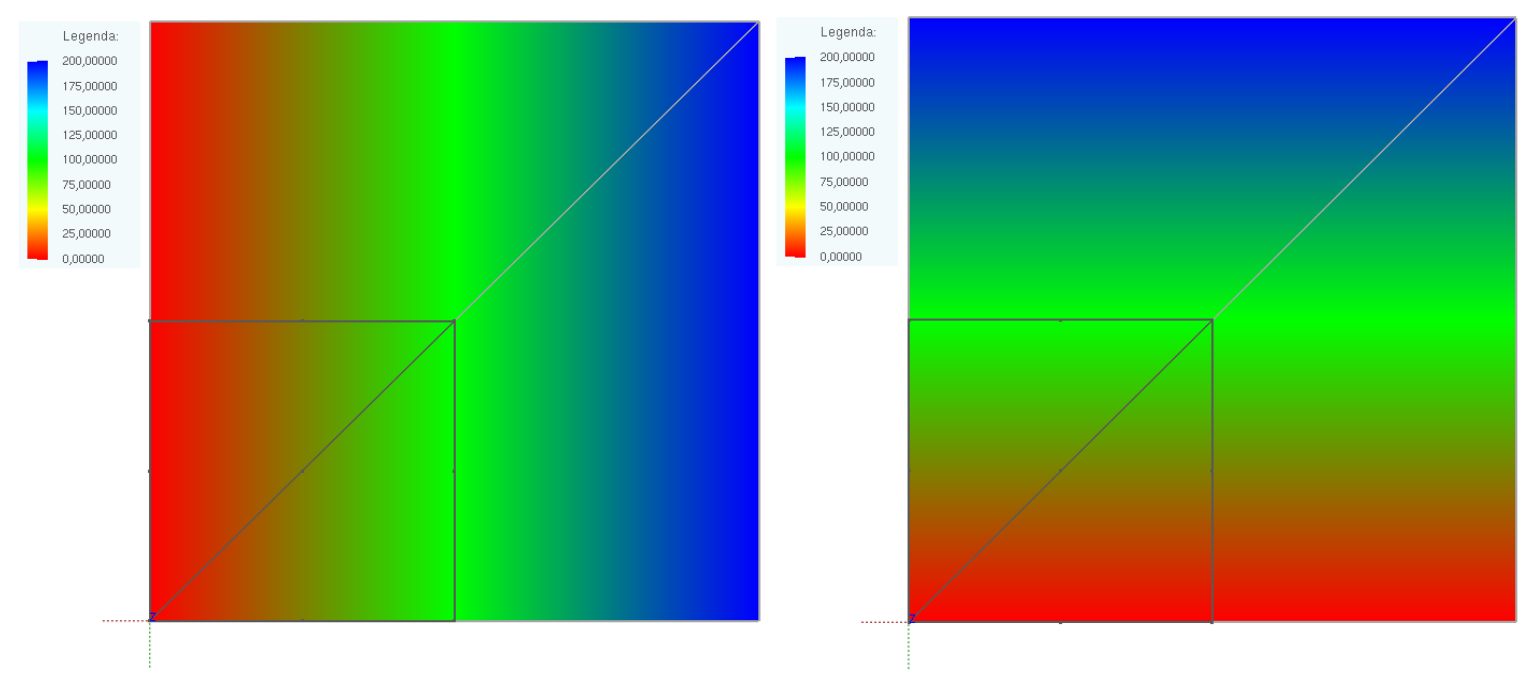

Figura 22 - Deslocamentos horizontais e verticais da chapa da Figura 21.
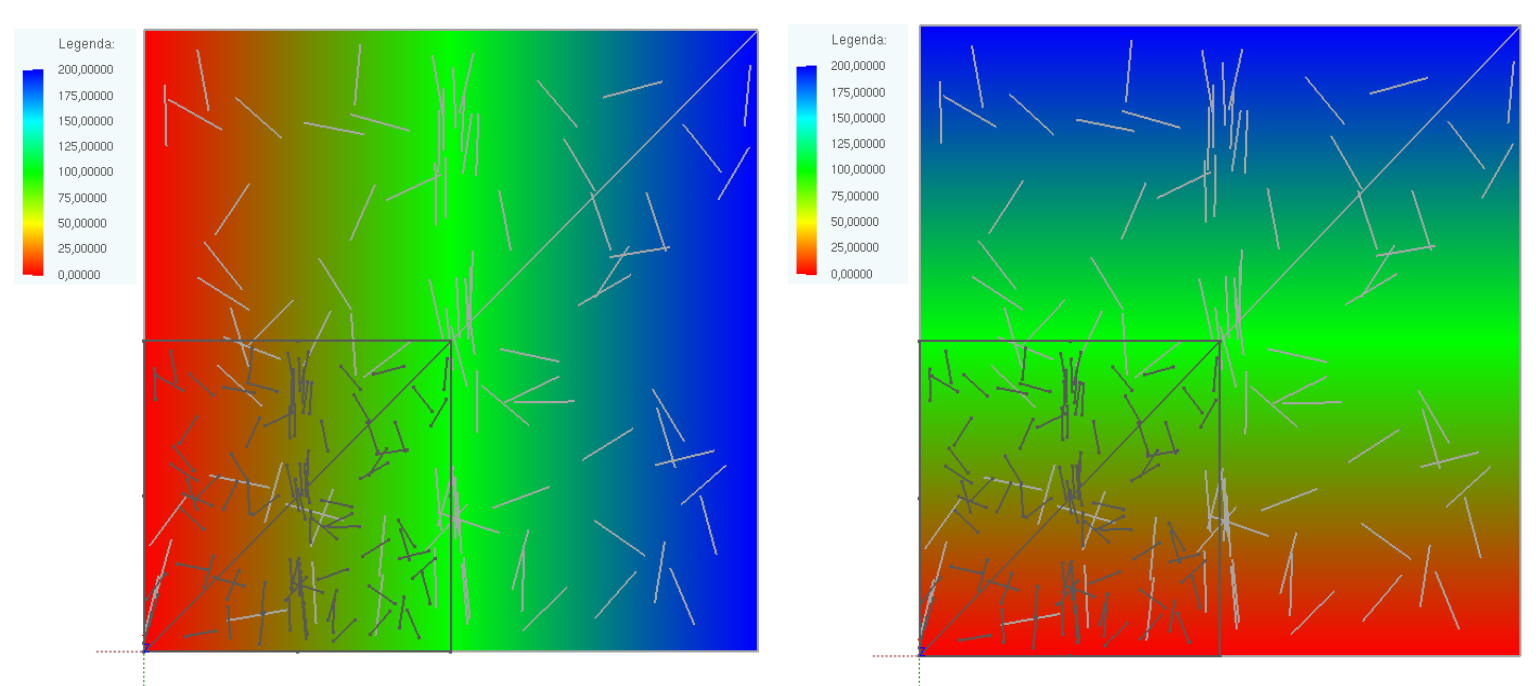

Figura 23 - Atualização das posições das fibras.

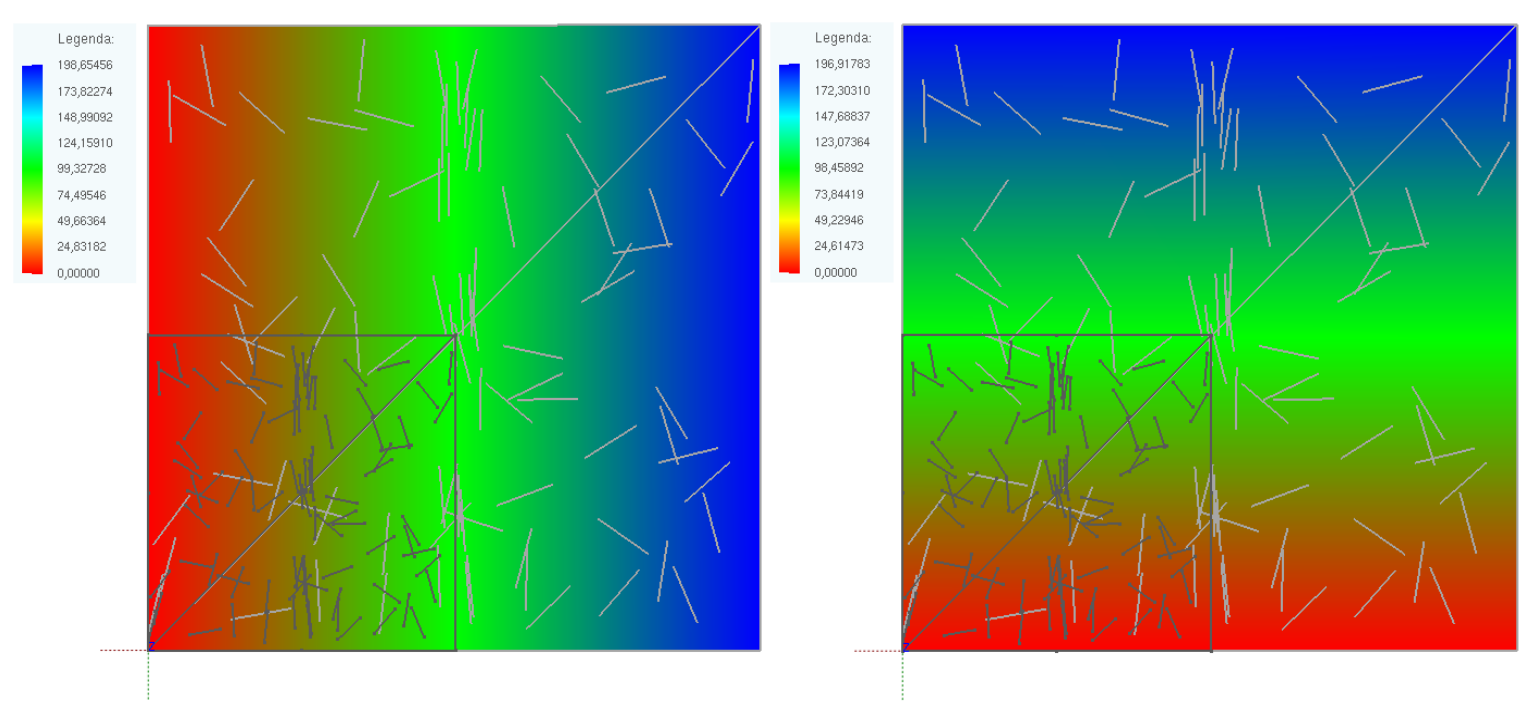

Figura 24 - Deslocamentos horizontais e verticais da chapa enrijecida com fibras aleatórias. 


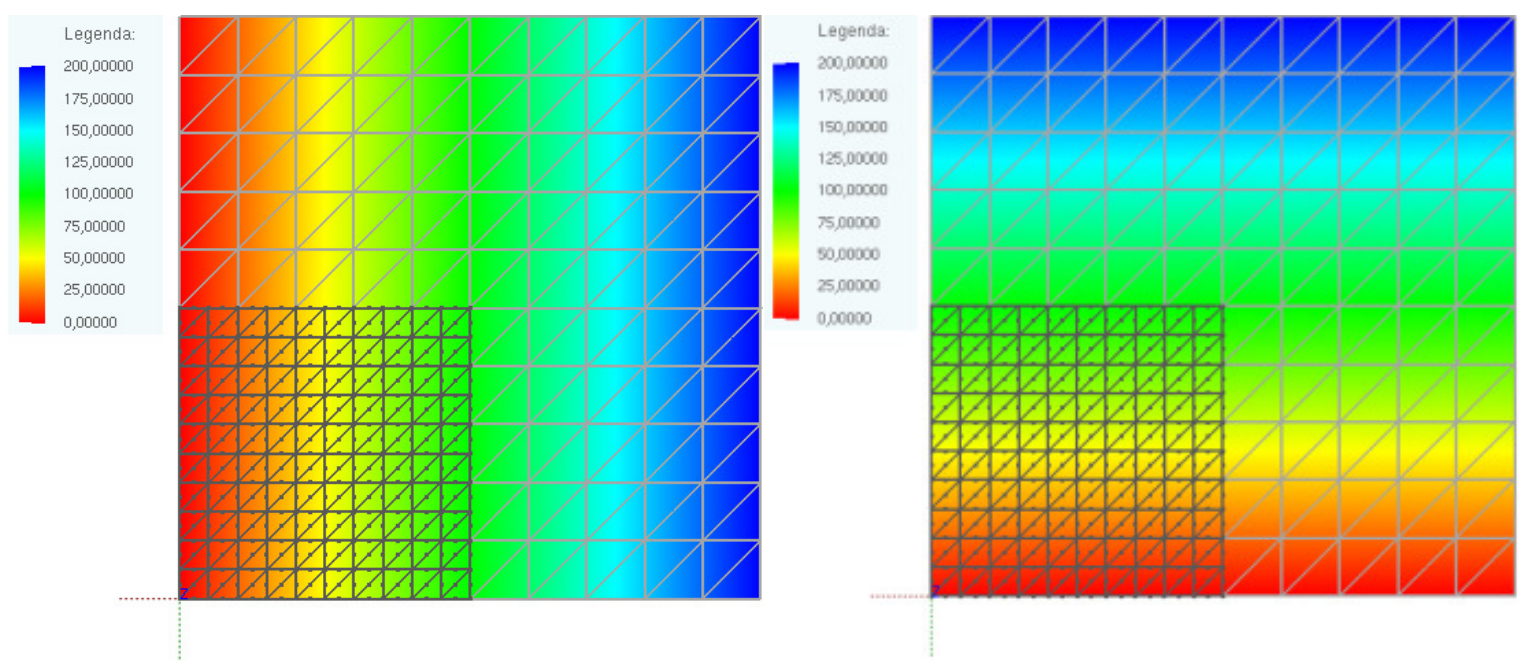

Figura 25 - Deslocamentos horizontais e verticais da chapa da Figura 21.

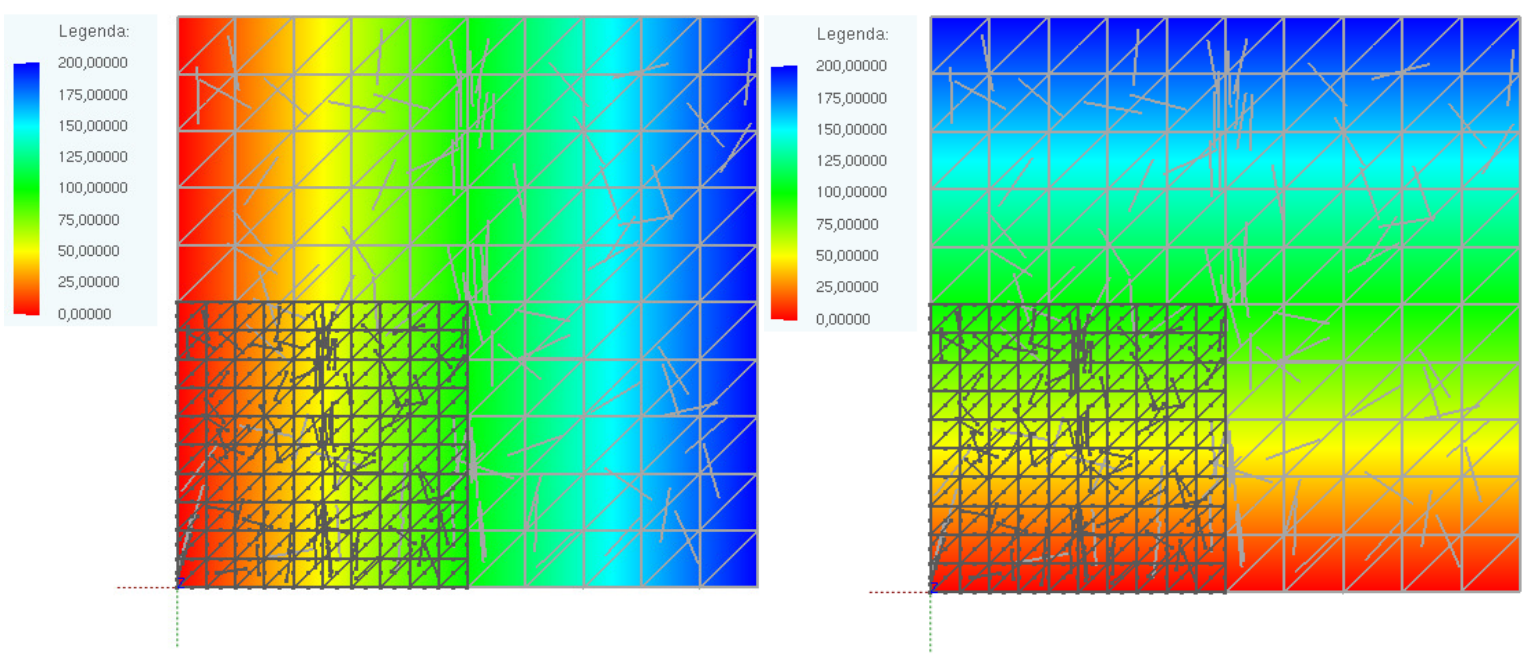

Figura 26 - Atualização das posições das fibras.

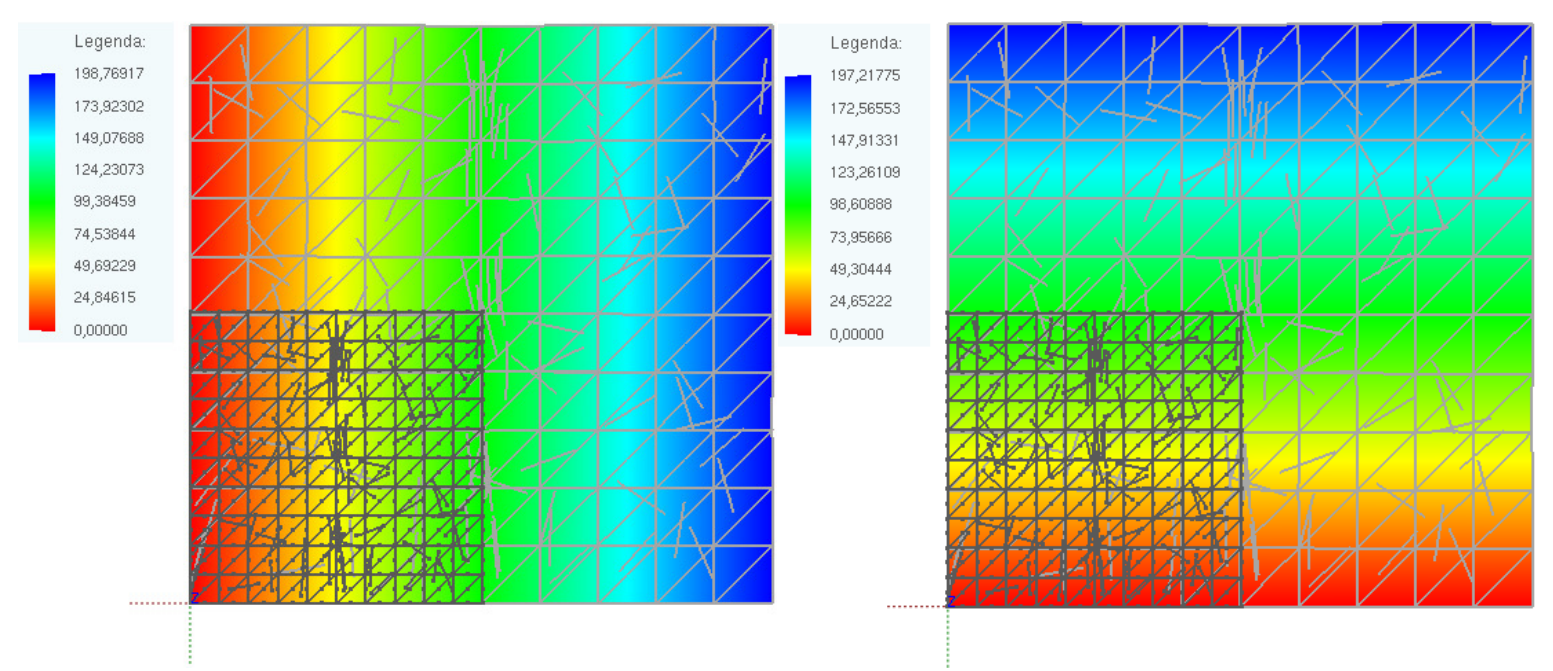

Figura 27 - Deslocamentos horizontais e verticais da chapa enrijecida com fibras aleatórias. 
Tabela 4 - Deslocamentos máximos da chapa para as diferentes análises realizadas em $(\mathrm{cm})$.

\begin{tabular}{|c|c|c|c|c|c|c|}
\hline \multirow{2}{*}{ DISCRETIZAÇÃOO } & \multicolumn{2}{|c|}{ HOMOGÊNEA } & \multicolumn{2}{|c|}{$E_{F}=0$} & \multicolumn{2}{|c|}{$E_{F}=10 N / \mathrm{cm}^{2}$} \\
\hline & $\mathbf{x}$ & $\mathbf{Y}$ & $\mathbf{x}$ & $\mathbf{Y}$ & $\mathbf{x}$ & $\mathbf{Y}$ \\
\hline \multirow{3}{*}{2 elementos } & \multirow{3}{*}{200,00} & \multirow{3}{*}{200,00} & \multirow{3}{*}{200,00} & \multirow{3}{*}{200,00} & 198,654 & 196,917 \\
\hline & & & & & & \\
\hline & & & & & 0,673 & 1,542 \\
\hline \multirow{3}{*}{200 elementos } & \multirow{3}{*}{200,00} & \multirow{3}{*}{200,00} & \multirow{3}{*}{200,00} & \multirow{3}{*}{200,00} & 198,769 & 197,217 \\
\hline & & & & & \multicolumn{2}{|c|}{ DESVIO (\%) } \\
\hline & & & & & 0,615 & 1,391 \\
\hline
\end{tabular}

Materiais com estas características são conhecidos como compósitos 2D ou compósitos randomicamente orientados no plano. Exemplos destes materiais são o Chopped Strand Mat (CSM) mostrado na Figura 28 e o Sheet Moulding Compounds (SMC) (MATTHEWS; RAWLINGS, 1994). Embora não seja o mais avançado, o CSM é um dos materiais compósitos mais utilizados na fabricação, reforço e laminação de componentes utilizados em diversas indústrias, tais como, automotiva, marítima, civil, etc.

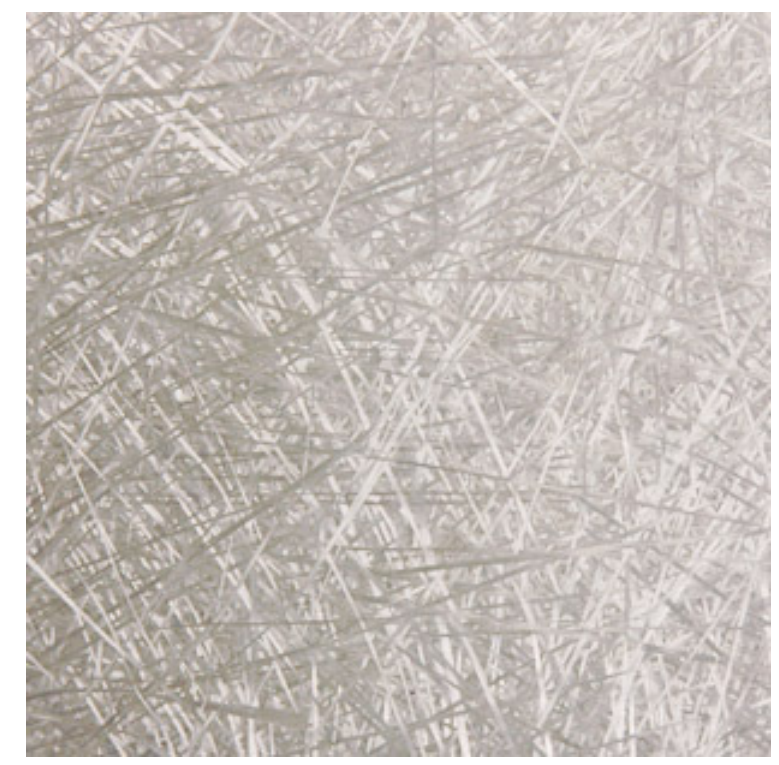

Figura 28 - Chopped strand mat (CSM). Fonte: http://www.easycomposites.co.uk/products/chopped-strandmat/powder-bound-chopped-strand-mat-300g-per-metre.aspx. Acesso em 01/11/2013. 


\subsubsection{EXEMPLO 4: CHAPA REFORÇADA COM FIBRA LONGA}

Neste exemplo, uma chapa quadrada de $(4 \times 4) \mathrm{mm}$ mostrada na Figura 29, originalmente apresentada por Radtke, Simone e Sluys (2010a) foi analisada. Este exemplo foi escolhido pelo reduzido número de variáveis envolvidas possibilitando a comparação com os resultados obtidos pelos autores de referência.

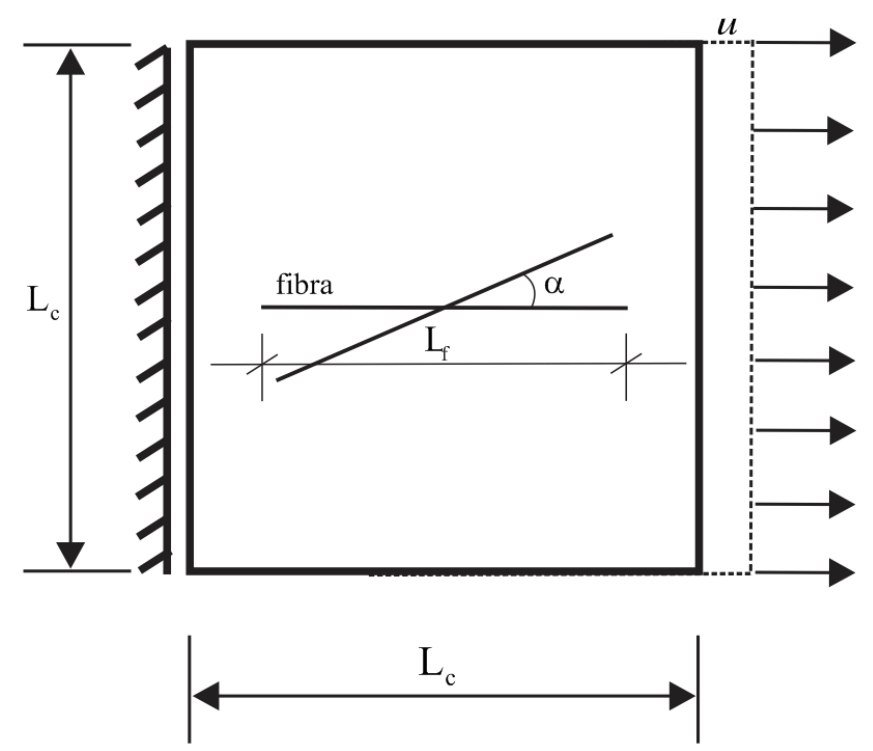

Figura 29 - Chapa quadrada reforçada com fibra e deslocamento imposto.

O material da matriz possui módulo de elasticidade $E_{m}=20 G P a$ e coeficiente de Poisson $v_{m}=0,2$. A fibra considerada como reforço tem $L_{f}=3 \mathrm{~mm}$ de comprimento e módulo de elasticidade $E_{f}=500 G P a$. A espessura da fibra é $d_{f}=0,05 \mathrm{~mm}$.

Nas análises realizadas neste trabalho, o módulo de elasticidade da matriz foi subtraído do módulo da fibra, pois ocupam o mesmo lugar no espaço. Para assegurar a análise não linear do problema e evitar diferenças entre deformação de engenharia e deformação de Green, o deslocamento aplicado foi dividido por mil e os valores obtidos em força foram multiplicados pela mesma quantidade.

A formulação apresentada por Radtke, Simone e Sluys (2010a) utiliza outras constantes relacionadas com a rigidez de contato fibra-matriz que não são aqui apresentadas, pois a formulação proposta no presente trabalho considera condições de contato perfeito. A extremidade direita da placa é tracionada por um deslocamento de $u=0,05 \mathrm{~mm}$ e a força de 
reação correspondente obtida é apresentada na Figura 30 para as diferentes discretizações utilizadas. Duas posições para a fibra foram consideradas, horizontal e inclinada, sendo que a última forma um ângulo de $30^{\circ}$ com a horizontal.

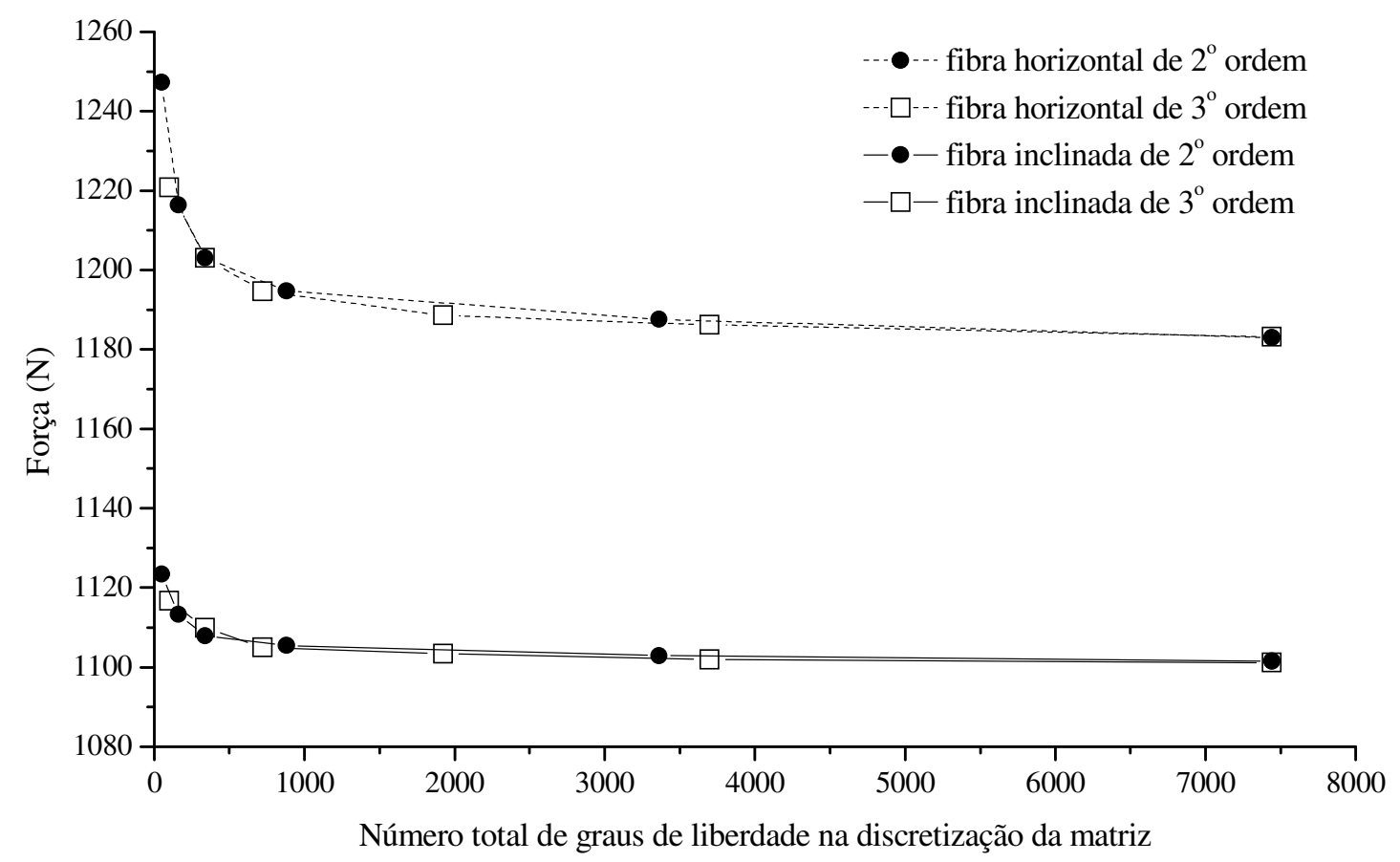

Figura 30 - Análise de convergência.

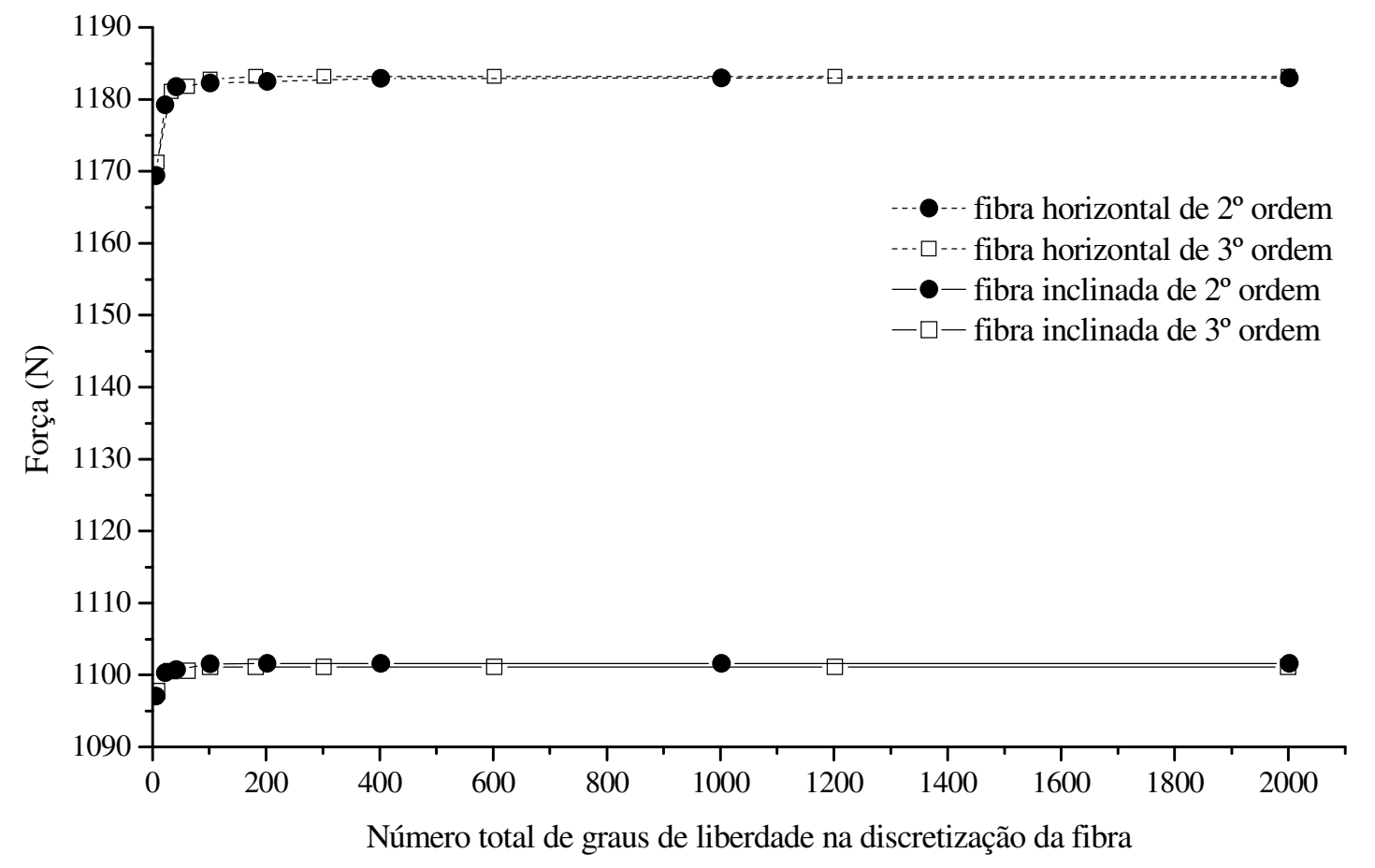

Figura 31 - Análise de dependência da discretização da fibra. 
A Figura 30 mostra a convergência dos resultados obtidos com elementos finitos quadráticos e cúbicos reforçados com fibras quadráticas e cúbicas, respectivamente. Para assegurar que a discretização das fibras não influencia nos resultados obtidos, a discretização, das fibras, com elementos quadráticos possui 500 elementos e 1001 nós e a discretização, das fibras, com elementos cúbicos tem 333 elementos e 1000 nós. Para discretizar a matriz foram utilizadas seis malhas quadrática com 8, 32, 72, 200, 800 e 1800 elementos e seis malhas cúbicas com 8, 32, 72, 200, 392 e 800 elementos. Os dados referentes às discretizações adotadas e os valores da força de reação correspondente para cada caso analisado são mostrados na Tabela 5. Destaca-se que o número de graus de liberdade total não é afetado pela discretização da fibra.

Os valores das forças obtidos por Radtke, Simone e Sluys (2010a) mostrados na Figura 32 são menores que os valores obtidos neste trabalho com desvio máximo de $2 \%$ para a fibra horizontal e de $\sim 0,5 \%$ para a fibra inclinada. Acredita-se que a diferença entre os resultados obtidos ocorre principalmente pela condição de contato fibra-matriz. Radtke, Simone e Sluys (2010a) adotam conexão elástica e neste trabalho adota-se conexão rígida.

Tabela 5 - Dados referentes à análise de convergência devido à discretização da malha da matriz.

\begin{tabular}{|c|c|c|c|c|c|c|c|c|c|c|c|}
\hline \multicolumn{6}{|c|}{ APROXIMAÇÃO DO $2^{\circ}$ GRAU } & \multicolumn{6}{|c|}{ APROXIMAÇÃO DO $3^{\circ}$ GRAU } \\
\hline \multirow[b]{2}{*}{ MALHA } & \multicolumn{3}{|c|}{ NÚMERO TOTAL } & \multicolumn{2}{|c|}{ FORÇA (N) } & \multirow[b]{2}{*}{ MALHA } & \multicolumn{3}{|c|}{ NÚMERO TOTAL } & \multicolumn{2}{|c|}{ FORÇA (N) } \\
\hline & Nós & ELEMENTOS & $\begin{array}{c}\text { GRAUS DE } \\
\text { LIBERDADE }\end{array}$ & \begin{tabular}{|c|} 
FIB RA \\
HORIZONTAL
\end{tabular} & $\begin{array}{c}\text { FIB RA } \\
\text { INCLINADA }\end{array}$ & & Nós & ELEMENTOS & $\begin{array}{c}\text { GRAUS DE } \\
\text { LIBERDADE }\end{array}$ & \begin{tabular}{|c|} 
FIB RA \\
HORIZONTAL
\end{tabular} & $\begin{array}{c}\text { FIB RA } \\
\text { INCLINADA }\end{array}$ \\
\hline 1 & 25 & 8 & 50 & 1247,3393 & 1123,5163 & 1 & 49 & 8 & 98 & 1220,8864 & 1116,7164 \\
\hline 2 & 81 & 32 & 162 & 1216,4354 & 1113,3673 & 2 & 169 & 32 & 338 & 1203,0790 & 1109,8933 \\
\hline 3 & 169 & 72 & 338 & 1203,0123 & 1107,8982 & 3 & 361 & 72 & 722 & 1194,6855 & 1104,9465 \\
\hline 4 & 441 & 200 & 882 & 1194,8322 & 1105,5204 & 4 & 961 & 200 & 1922 & 1188,5744 & 1103,4072 \\
\hline 5 & 1681 & 800 & 3362 & 1187,6122 & 1102,8744 & 5 & 1849 & 392 & 3698 & 1186,2091 & 1101,8926 \\
\hline 6 & 3721 & 1800 & 7442 & 1182,9726 & 1101,5823 & 6 & 3721 & 800 & 7442 & 1183,1528 & 1101,1088 \\
\hline
\end{tabular}

Tabela 6 - Dados referentes à análise da influência da discretização das fibras na solução.

\begin{tabular}{|c|c|c|c|c|c|c|c|c|c|c|c|}
\hline \multicolumn{6}{|c|}{ APROXIMAÇÃO DO $2^{\circ}$ GRAU } & \multicolumn{6}{|c|}{ APROXIMAÇÃO DO $3^{\circ}$ GRAU } \\
\hline \multirow{2}{*}{ MALHA } & \multicolumn{3}{|c|}{ NÚMERO TOTAL } & \multicolumn{2}{|c|}{ FORÇA (N) } & \multirow{2}{*}{ MALHA } & \multicolumn{3}{|c|}{ NÚMERO TOTAL } & \multicolumn{2}{|c|}{ FORÇA (N) } \\
\hline & Nós & ELEMENTOS & \begin{tabular}{|c|} 
GRAUS DE \\
LIBERDADE
\end{tabular} & $\begin{array}{c}\text { FIBRA } \\
\text { HORIZONTAL }\end{array}$ & \begin{tabular}{|c|} 
FIB RA \\
INCLINADA
\end{tabular} & & Nós & ELEMENTOS & \begin{tabular}{|c|} 
GRAUS DE \\
LIBERDADE
\end{tabular} & $\begin{array}{c}\text { FIBRA } \\
\text { HORIZONTAL }\end{array}$ & \begin{tabular}{|c|} 
FIBRA \\
INCLINADA
\end{tabular} \\
\hline 1 & 3 & 1 & 6 & 1169,3786 & 1097,0646 & 1 & 4 & 1 & 8 & 1171,2433 & 1097,6973 \\
\hline 2 & 11 & 5 & 22 & 1179,2248 & 1100,2675 & 2 & 16 & 5 & 32 & 1181,0762 & 1100,4677 \\
\hline 3 & 21 & 10 & 42 & 1181,7257 & 1100,7169 & 3 & 51 & 20 & 102 & 1182,8088 & 1101,0991 \\
\hline 4 & 51 & 25 & 102 & 1182,2415 & 1101,5043 & 4 & 91 & 30 & 182 & 1183,1544 & 1101,1062 \\
\hline 5 & 101 & 50 & 202 & 1182,4541 & 1101,5705 & 5 & 151 & 50 & 302 & 1183,1547 & 1101,1095 \\
\hline 6 & 201 & 100 & 402 & 1182,9156 & 1001,5767 & 6 & 301 & 100 & 602 & 1183,1532 & 1101,1088 \\
\hline 7 & 501 & 250 & 1002 & 1182,9379 & 1101,5836 & 7 & 601 & 200 & 1202 & 1183,1572 & 1101,1088 \\
\hline 8 & 1001 & 500 & 2002 & 1182,9726 & 1101,5823 & 8 & 1000 & 333 & 2000 & 1183,1528 & 1101,1088 \\
\hline
\end{tabular}

Embora o número de graus de liberdade das fibras não afete o número de graus de liberdade do problema, este parâmetro foi utilizado para mostrar a dependência da solução 
em relação à discretização adotada para a fibra. Para esta análise utilizou-se a malha mais refinada da análise anterior para discretizar a matriz, ou seja, 1800 elementos para o caso quadrático e 800 elementos para o caso cúbico e variou-se a malha da fibra em ambos os casos. Para o caso quadrático as malhas utilizadas para discretizar as fibras possuem 1, 5, 10, 25, 50, 100, 250 e 500 e 1, 5, 20, 30, 50, 100, 200 e 333 elementos para o caso cúbico. Os dados referentes às discretizações adotadas e os valores da força de reação correspondente para cada caso analisado são mostrados na Tabela 6 e as curvas obtidas com estes dados são mostradas na Figura 31. É possível observar, da Figura 31, que a influência da discretização das fibras na força de reação é pequena, entretanto, a utilização de elementos finitos de fibra com comprimento igual à dimensão do lado do elemento finito de chapa garante a conformidade da discretização e melhora o comportamento global do sistema. É importante observar que ao utilizar poucos elementos de fibra o comportamento obtido é mais flexível, pois a taxa de transferência de força da matriz para a fibra é menor que aquela observada para um modelo com uma discretização mais densa.

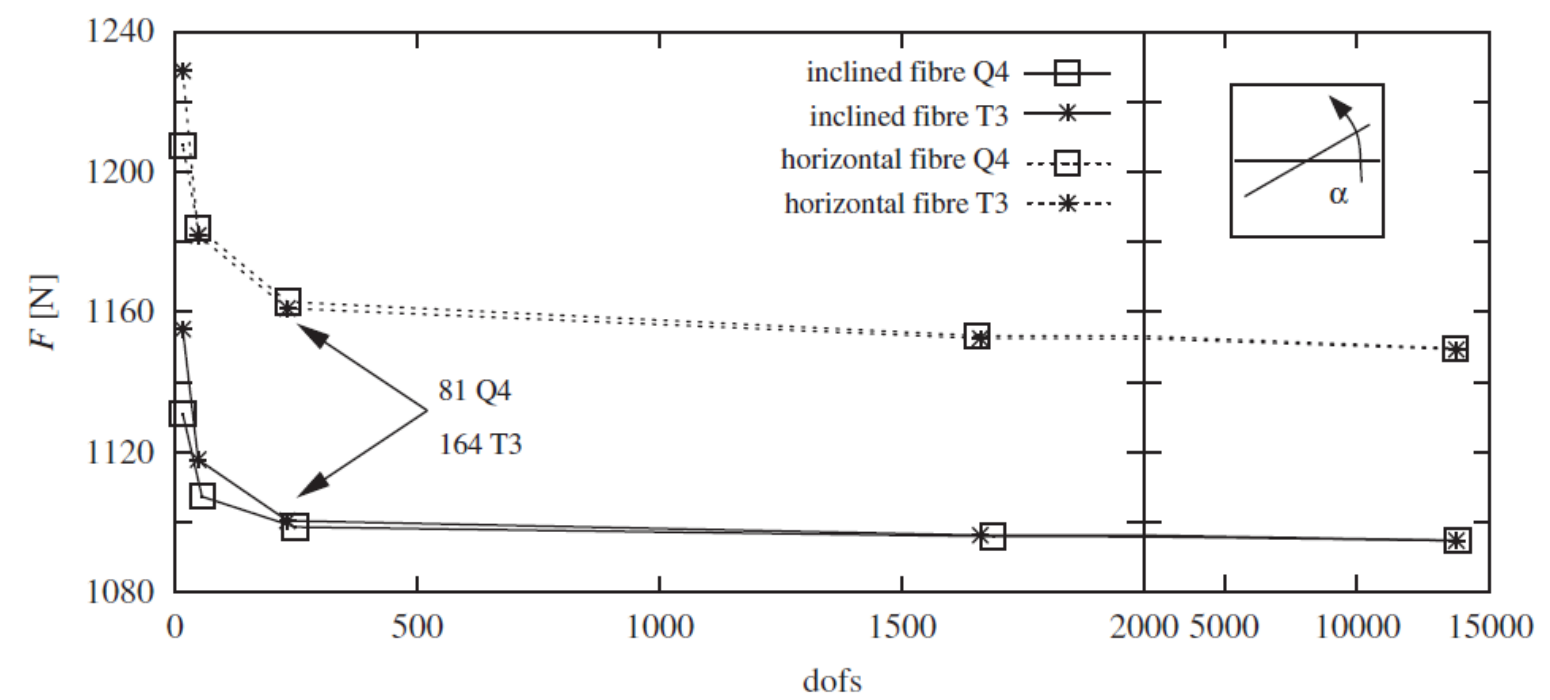

Figura 32 - Resultados de Radtke, Simone e Sluys (2010a) para a análise de convergência da força de reação devido à discretização da matriz. Fonte: Radtke, Simone e Sluys (2010a). 


\subsubsection{EXEMPLO 5: CHAPA REFORÇADA COM FIBRAS CURVAS}

O objetivo deste exemplo é analisar a convergência do campo de tensões desenvolvido pelo domínio bidimensional quando reforçado com fibras que possuem trechos curvos em suas extremidades. A tensão existente num corpo submetido a um carregamento qualquer considerada como verdadeira é aquela dada pelo tensor das tensões de Cauchy, $\hat{\boldsymbol{\Gamma}}$, que relaciona as forças na configuração final deformada com as áreas da configuração final deformada. Uma vez conhecido o tensor tensão de Piola-Kirchhoff de segunda espécie, $\hat{\boldsymbol{S}}=\frac{\partial \hat{w}}{\partial \hat{\boldsymbol{E}}}$, o tensor das tensões de Cauchy, $\hat{\boldsymbol{\Gamma}}$, pode ser obtido a partir da relação:

$$
\hat{\boldsymbol{\Gamma}}=\frac{\hat{\boldsymbol{A}} \hat{\boldsymbol{S}} \hat{\boldsymbol{A}}^{T}}{\hat{\boldsymbol{J}}}
$$

onde $\hat{J}=\operatorname{det}(\hat{\boldsymbol{A}})$ é o Jacobiano da transformação, $\hat{A}$ é o tensor gradiente da função mudança de configuração dado na Eq.(4.4) e $\hat{\boldsymbol{A}}^{T}$ é o tensor gradiente da função mudança de configuração transposto.

Para tanto, realiza-se um ensaio de tração da chapa reforçada com fibras mostrada na Figura 33. Os trechos curvos das fibras possuem raio $R_{f}=0,5 \mathrm{~cm}$. O módulo de elasticidade da matriz é $E_{m}=1 G P a$ e o coeficiente de Poisson $v_{m}=0,0$. O módulo de elasticidade das fibras e a área da seção transversal são dados, respectivamente, por $E_{f}=20,0 G P a$ $E_{f}=20,0 G P a$ e $A_{f}=0,1 \mathrm{~cm}^{2}$.

Devido à dupla simetria somente um quarto do problema foi discretizado. Um deslocamento total de $u=0,5 \mathrm{~cm}$ foi imposto na extremidade esquerda do quarto da chapa cujas dimensões são apresentadas na Figura 33 e na Figura 34. A matriz foi discretizada em três diferentes malhas com 1000, 4000 e 9000 elementos finitos triangulares cúbicos, mostradas na Figura 35, na Figura 36 e na Figura 37, respectivamente, e a fibra foi discretizada em 150 elementos cúbicos no trecho horizontal e 20 elementos finitos cúbicos no trecho curvo. 
A Figura 38 mostra a distribuição para a componente $\hat{\Gamma}_{11}$ da tensão de Cauchy em uma porção retangular da chapa com dimensões de $2,4 \mathrm{~cm}$ na direção horizontal e $3,0 \mathrm{~cm}$ na direção vertical localizada em uma região próxima ao trecho curvo da fibra. Apresenta-se um gráfico descontínuo em que os valores em azul representam tensões acima de 93,60MPa e valores em vermelho representam tensões inferiores a 2,84MPa. É possível observar que as variações entre os valores obtidos para a segunda e a terceira malha são pequenas caracterizando a convergência do campo de tensões para todos os pontos da chapa exceto para uma região próxima à ponta da fibra.

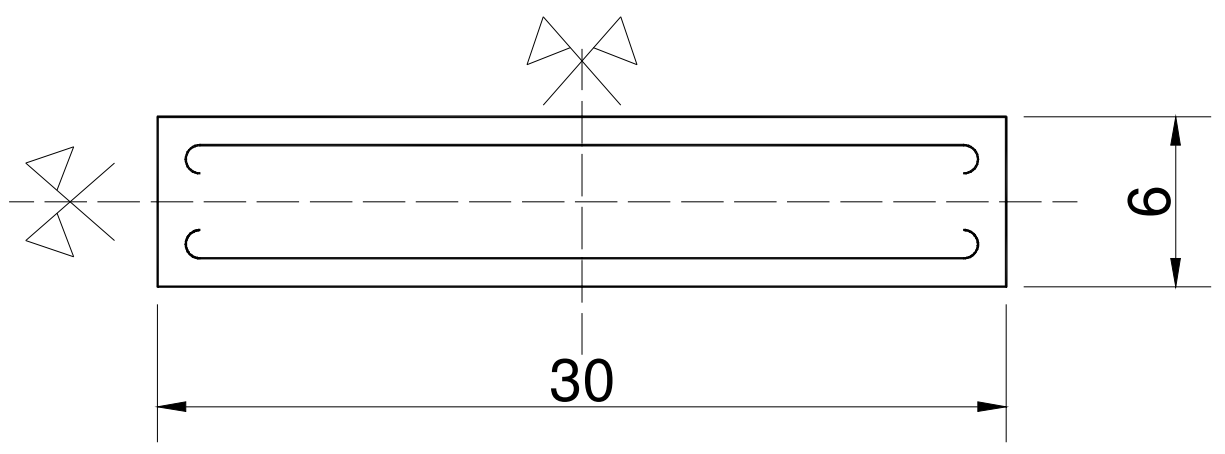

Figura 33 - Chapa reforçado com fibras com extremidades curvas em (cm).

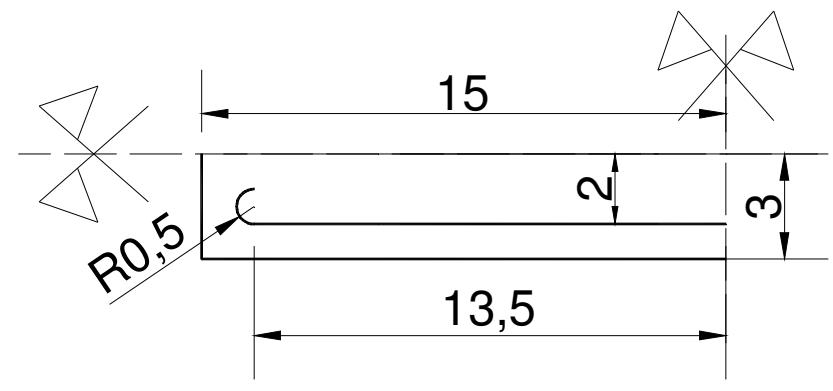

Figura 34 - Dimensões de um quarto da chapa em $(\mathrm{cm})$.

Ao ampliar os gráficos é possível verificar que há concentração de tensão na extremidade da fibra. Os valores máximos da tensão horizontal para cada discretização são respectivamente, $\hat{\Gamma}_{11}^{1}=93,60 \mathrm{MPa}, \hat{\Gamma}_{11}^{2}=109,09 \mathrm{MPa}$ e $\hat{\Gamma}_{11}^{3}=118,40 \mathrm{MPa}$, concentrados em uma extensão muito pequena do domínio medindo aproximadamente $0,03 \mathrm{~cm}$ ou um terço da dimensão do lado do menor elemento finito bidimensional. Estudos futuros considerando não linearidade material e outras condições de contato fibra-matriz devem ser realizados com a formulação proposta objetivando melhorias na taxa de convergência observada.

A Figura 39 mostra os deslocamentos horizontais para um quarto da chapa reforçada com fibras com extremidades curvas onde é possível observar a influência global do reforço 
na solução.

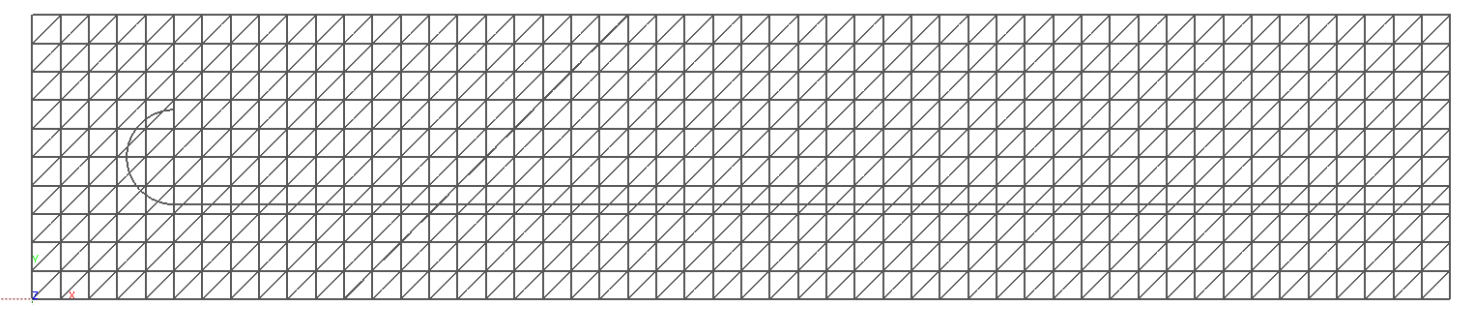

Figura 35 - Malha 1: 1000 elementos triangulares cúbicos totalizando 9362 graus de liberdade.

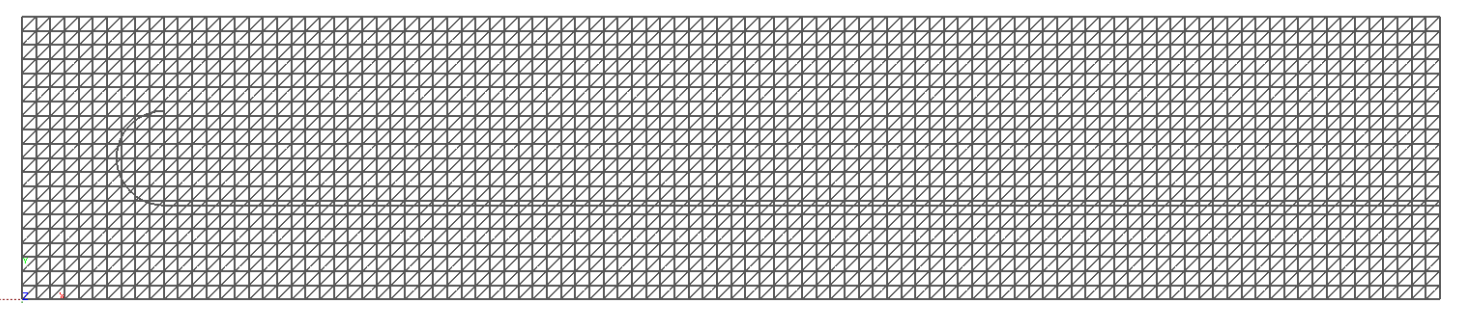

Figura 36 - Malha 2: 4000 elementos triangulares cúbicos totalizando 36722 graus de liberdade.

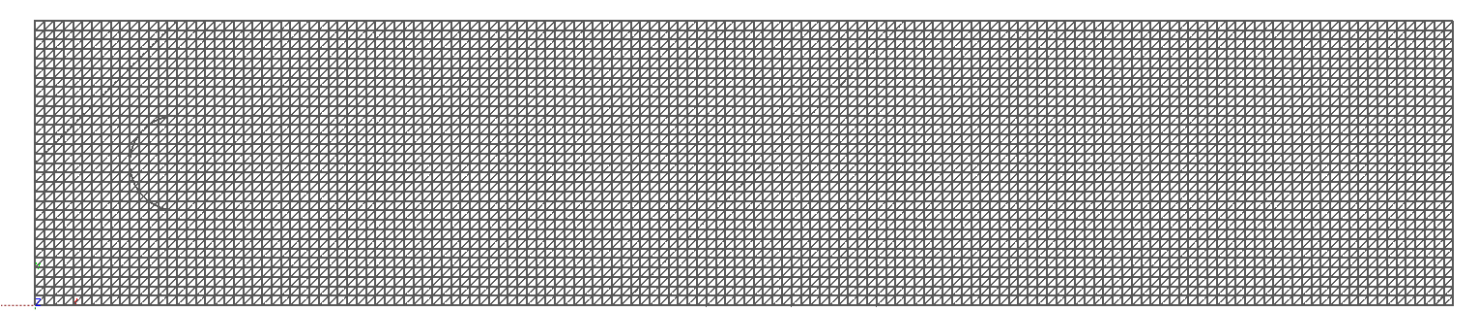

Figura 37 - Malha 3: 9000 elementos triangulares cúbicos totalizando 82082 graus de liberdade.
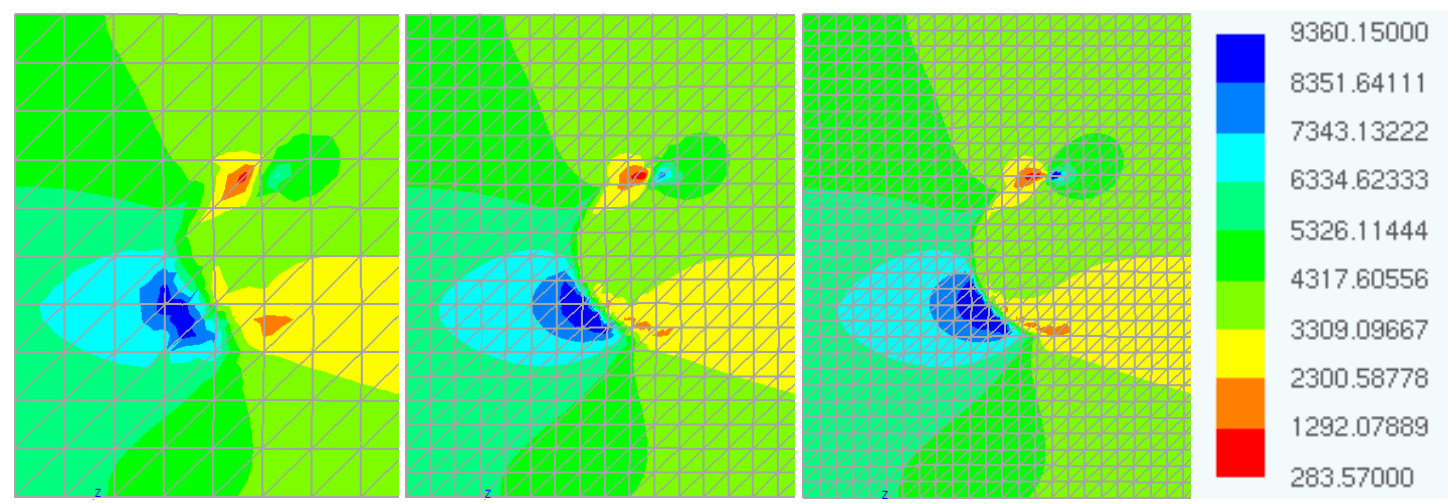

Figura 38 - Componente $\hat{\Gamma}_{11}$ da Tensão de Cauchy para as diferentes discretizações adotadas (unidades em 10MPa).

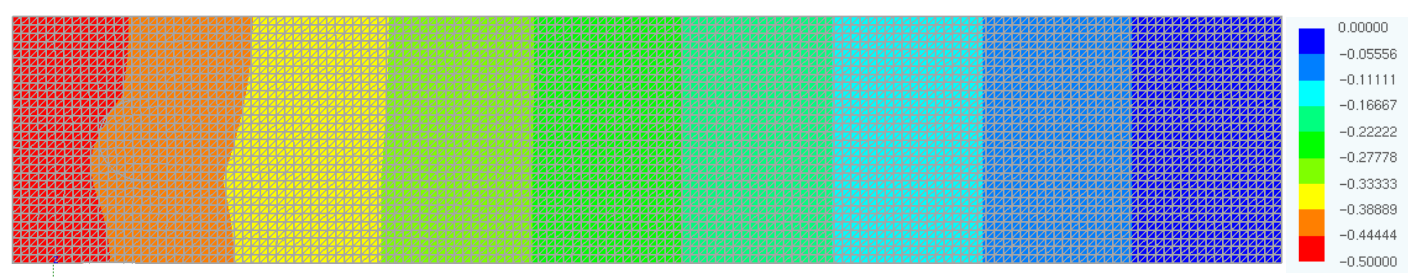

Figura 39 - Deslocamentos horizontais em $(\mathrm{cm})$. 


\section{ANÁLISE DE TENSÃO FIBRA-MATRIZ EM MEIOS BIDIMENSIONAIS}

\subsection{CONSIDERAÇÕES INICIAIS}

Em condições de contato perfeito e deformações elásticas, a distribuição das tensões ao longo de uma fibra alinhada paralelamente à direção de aplicação de um carregamento na matriz considerada (Figura 40) pode ser representada conforme mostrado na Figura 41, ou seja, a tensão normal é nula nas extremidades da fibra e máxima no centro, e a tensão de cisalhamento é máxima nas extremidades e praticamente nula no centro da fibra (MATTHEWS; RAWLINGS, 1994).

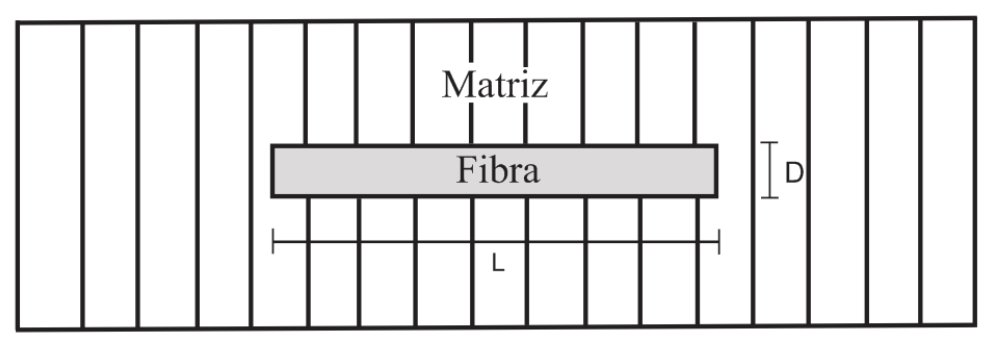

Indeformada

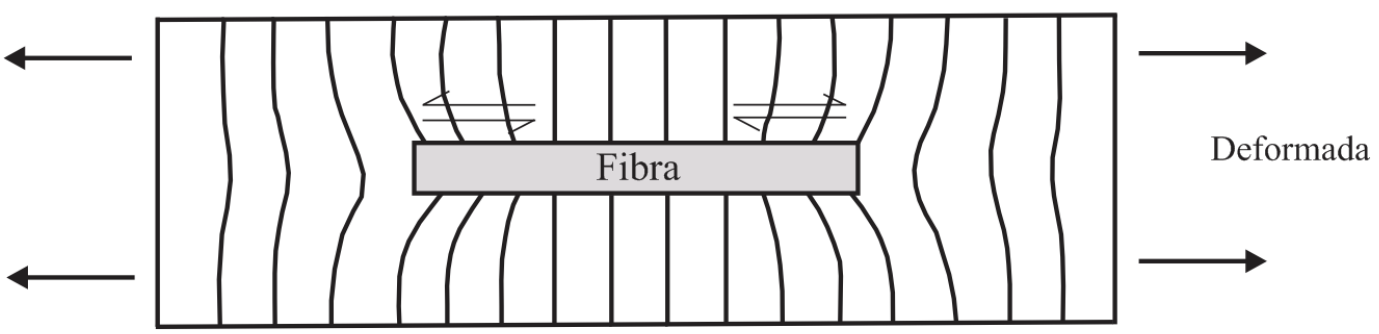

Figura 40 - Efeito da deformação de uma matriz com baixo módulo de elasticidade na fibra. Fonte: (MATTHEWS; RAWLINGS, 1994).

Em trechos de fibra curvos, além da tensão normal ao longo da fibra e da tensão de cisalhamento na interface, surge uma terceira tensão na direção do vetor $\vec{N}$ mostrado na Figura 42, denominada aqui como tensão normal de contato. Lembrando-se que $\vec{N}$ forma um 
ângulo reto com o vetor tangente $\vec{T}$ e $\vec{B}$ é o vetor binormal perpendicular ao vetores $\vec{T}$ e $\vec{N}$ muito útil ao se trabalhar com fibras no espaço tridimensional.

Como exemplo prático de trechos de fibra curvos citam-se os ganchos empregados para ancorar as barras de aço no concreto armado e melhorar a condição de aderência.

Uma das vantagens da formulação introduzida neste trabalho para a discretização das fibras independentemente da matriz é a possibilidade de determinação da distribuição das tensões na fibra.

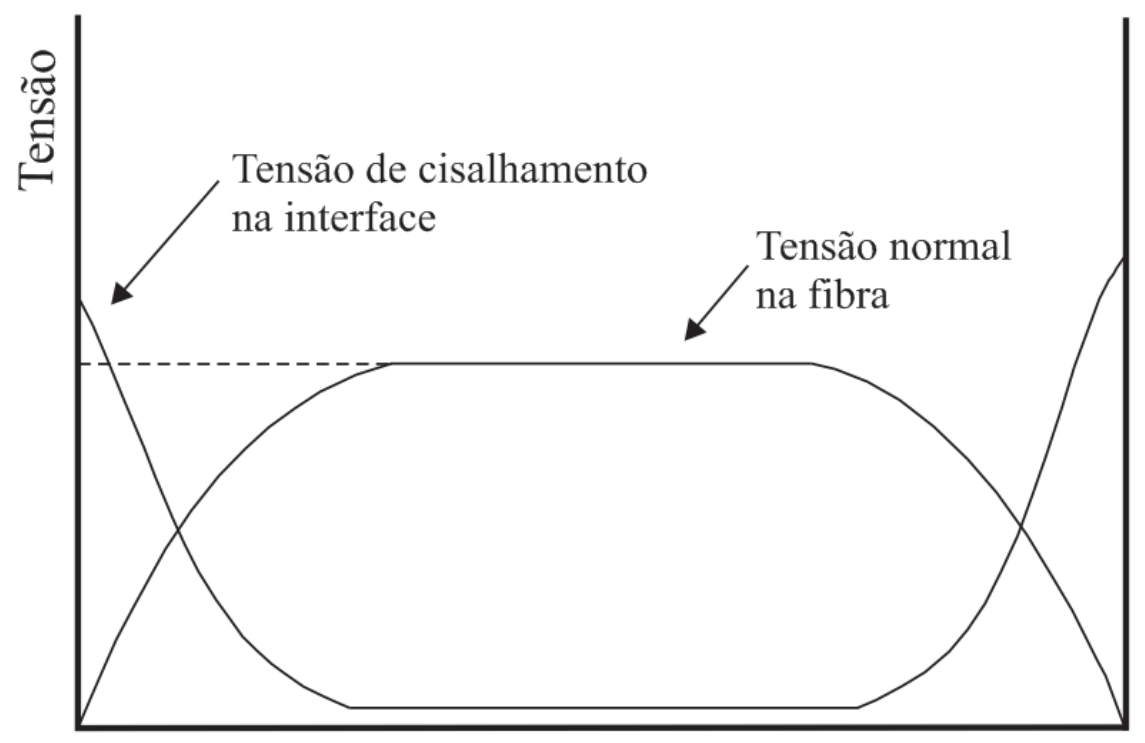

Fibra

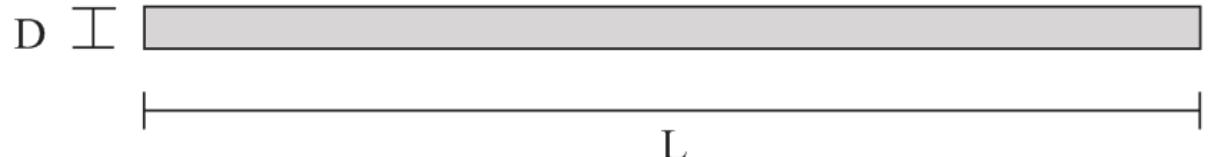

Figura 41 - Distribuição de tensão normal na fibra e de cisalhamento na interface fibra-matriz. Fonte: (MATTHEWS; RAWLINGS, 1994).

Desta forma, descrevem-se neste capítulo, duas estratégias adotadas para a determinação das tensões de contato ao longo das fibras curvas de ordem qualquer introduzidas neste trabalho.

A primeira estratégia, obtida a partir de relações diferenciais, é apresentada na Seção 5.2. Na segunda estratégia, descrita na Seção 5.3, a transferência das forças nodais da fibra para a matriz é decomposta segundo as direções normal e tangencial ao nó da fibra e dividida por uma porção do comprimento da fibra, denominado comprimento de influência. Esta estratégia é aqui designada como formulação média. 
Exemplos numéricos utilizados para validar as estratégias introduzidas são apresentados na Seção 5.4.

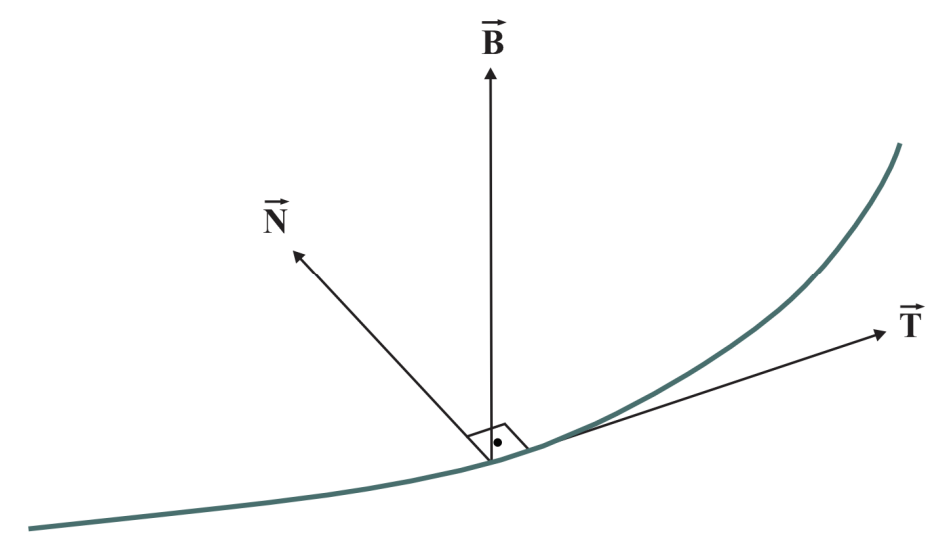

Figura 42 - Vetores associados a um nó de um trecho de fibra curvo.

\subsection{FORMULAÇÃO DIFERENCIAL}

\subsubsection{TENSÃO DE ADERÊNCIA}

A Figura 43 mostra um elemento diferencial de fibra curva de ordem qualquer com comprimento diferencial da coordenada curvilínea ao longo da fibra deformada $d \tilde{s}$ e espessura $\tilde{t}$. Da condição de equilíbrio na direção da fibra, tem-se que a tensão de aderência $\sigma_{a}^{d}(\eta)$ é dada por:

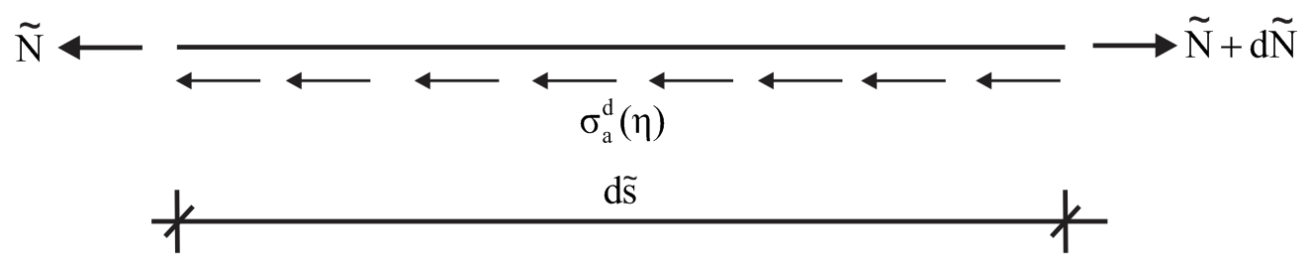

Figura 43 - Equilíbrio diferencial na direção da fibra.

$$
\sigma_{a}^{d}=\frac{1}{\tilde{t}} \frac{d \tilde{N}}{d \tilde{s}}
$$


O comprimento diferencial $d \tilde{s}$ é calculado como uma função das coordenadas adimensionais $\eta$ pela expressão:

$$
d \tilde{s}=\sqrt{d \tilde{y}_{1}^{2}+d \tilde{y}_{2}^{2}}=\sqrt{\left(\frac{d \tilde{y}_{1}}{d \eta} d \eta\right)^{2}+\left(\frac{d \tilde{y}_{2}}{d \eta} d \eta\right)^{2}}=\tilde{J}(\eta) d \eta,
$$

ou simplesmente,

$$
\frac{d \tilde{s}}{d \eta}=\tilde{J}(\eta)
$$

Aplicando a regra da cadeia no termo $\frac{d \tilde{N}}{d \tilde{s}}$ da Eq. (5.1) e usando a Eq.(5.3), tem-se que:

$$
\frac{d \tilde{N}}{d \tilde{s}}=\frac{d \tilde{N}}{d \eta} \frac{d \eta}{d \tilde{s}}=\frac{d \tilde{N}}{d \eta} \frac{1}{\frac{d \tilde{s}}{d \eta}}=\frac{1}{\tilde{J}(\eta)} \frac{d \tilde{N}}{d \eta}
$$

Considerando que a espessura $\tilde{t}$ da fibra é constante, a componente normal da Tensão de Cauchy no interior da fibra, $\sigma(\eta)$, é calculada a partir da Tensão de Piola-Kirchhoff, $\tilde{S}(\eta)$, pela expressão:

$$
\sigma(\eta)=\frac{\tilde{J}(\eta)}{\tilde{J}_{0}(\eta)} \tilde{S}(\eta)=\frac{\tilde{J}(\eta)}{\tilde{J}_{0}(\eta)} \tilde{\mathbb{E}} \tilde{E}(\eta)
$$

e, consequentemente, a Força Normal na fibra curva, $\tilde{N}(\eta)$, é dada por:

$$
\tilde{N}(\eta)=\frac{\tilde{J}(\eta)}{\tilde{J}_{0}(\eta)} \tilde{\mathbb{E}} \tilde{E}(\eta) \tilde{A}
$$

$\operatorname{com} \tilde{E}(\eta)$ é dada pela Eq. (4.26).

Em vez de diferenciar a Eq. (5.6) em relação a $\eta$ e substituir na Eq. (5.4), e o 
resultado desta na Eq. (5.1), calculam-se os valores nodais da força normal usando a Eq. (5.6) e faz-se:

$$
\tilde{N}(\eta)=\tilde{\phi}_{p}(\eta) \tilde{N}^{p}
$$

portanto,

$$
\frac{d \tilde{N}}{d \eta}(\eta)=\frac{d \tilde{\phi}_{p}(\eta)}{d \eta} \tilde{N}^{p}
$$

Substituindo a Eq. (5.8) na Eq. (5.4) e a Eq. (5.4) na Eq. (5.1) resulta que a tensão de aderência pode ser calculada como:

$$
\sigma_{a}^{d}(\eta)=\frac{1}{\tilde{t}} \frac{1}{\tilde{J}(\eta)} \frac{d \tilde{\phi}_{p}(\eta)}{d \eta} \tilde{N}^{p}
$$

Da forma como apresentada, a Eq. (5.7) permite que a ordem de aproximação de $\tilde{N}(\eta)$, no cálculo da Eq. (5.9), seja diferente daquela adotada para a aproximação das posições dos nós do elemento de fibra curvo.

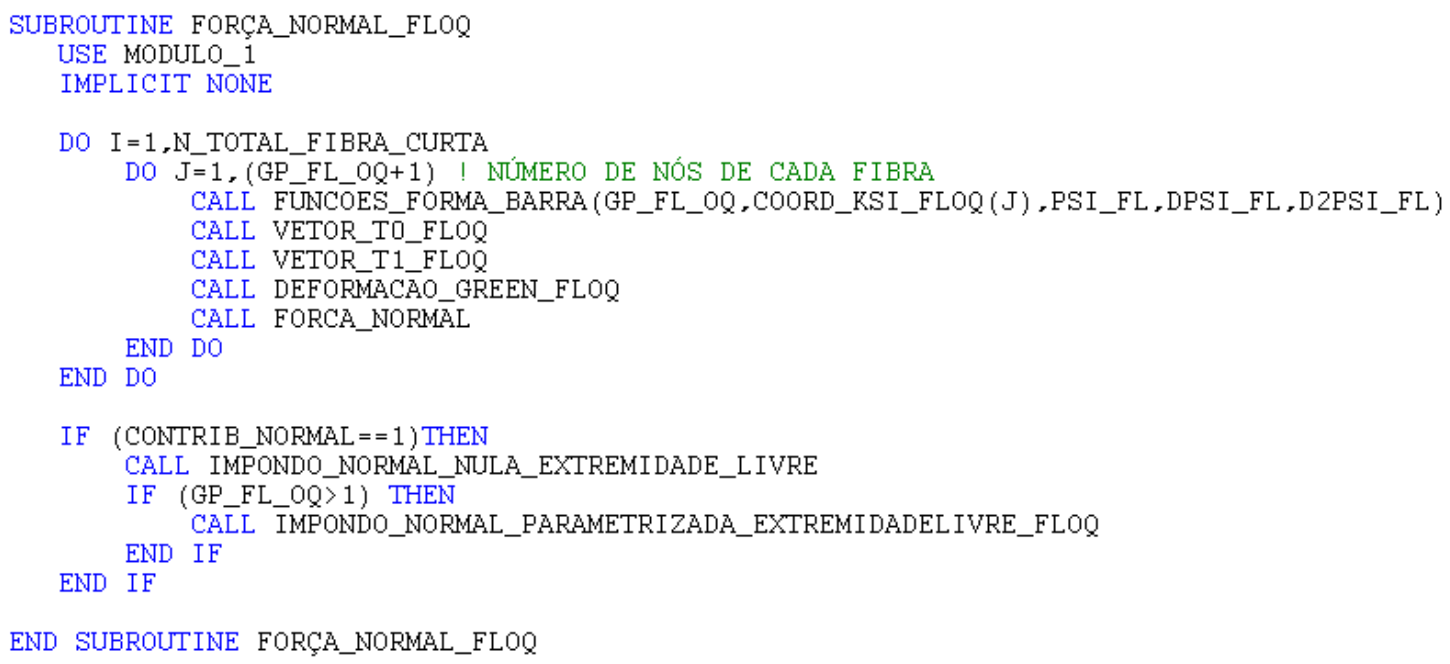

Figura 44 - Sub-rotina principal que gerencia a determinação da força normal nas fibras.

Uma característica desta formulação, observada nas análises numéricas, é a presença 
de um valor significativo de força normal nas extremidades dos enrijecedores revelando que o uso direto da Eq. (5.9) permite transferência de força por tensão normal nas extremidades dos elementos. Para remover este mecanismo de transferência é necessário impor $\tilde{N}=0$ nas extremidades das barras na Eq. (5.9). Com isto, a transferência de força pela ponta ocorre apenas por tensão de aderência.

A Figura 44 mostra o aspecto geral da sub-rotina principal que gerencia a obtenção da força normal nas fibras e a Figura 45 mostra a sub-rotina principal que gerencia a obtenção da tensão de aderência nas fibras.

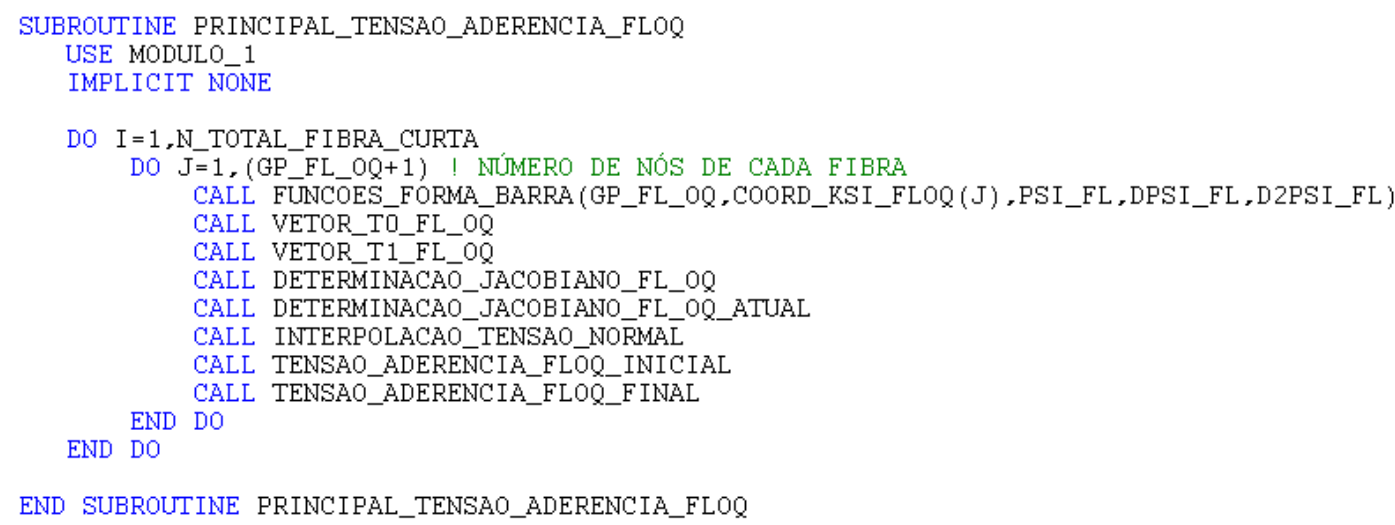

END SUBROUTINE PRINCIPAL_TENSAO_ADERENCIA_FLOQ

Figura 45 - Sub-rotina principal que gerencia a determinação da tensão de aderência nas fibras.

\subsubsection{TENSÃO NORMAL DE CONTATO}

Para determinar a expressão da tensão normal de contanto considera-se um elemento diferencial de fibra curva de ordem qualquer, $d \tilde{s}$, submetido a um carregamento distribuído $\sigma_{n c}^{d}$, conforme mostrado na Figura 46.

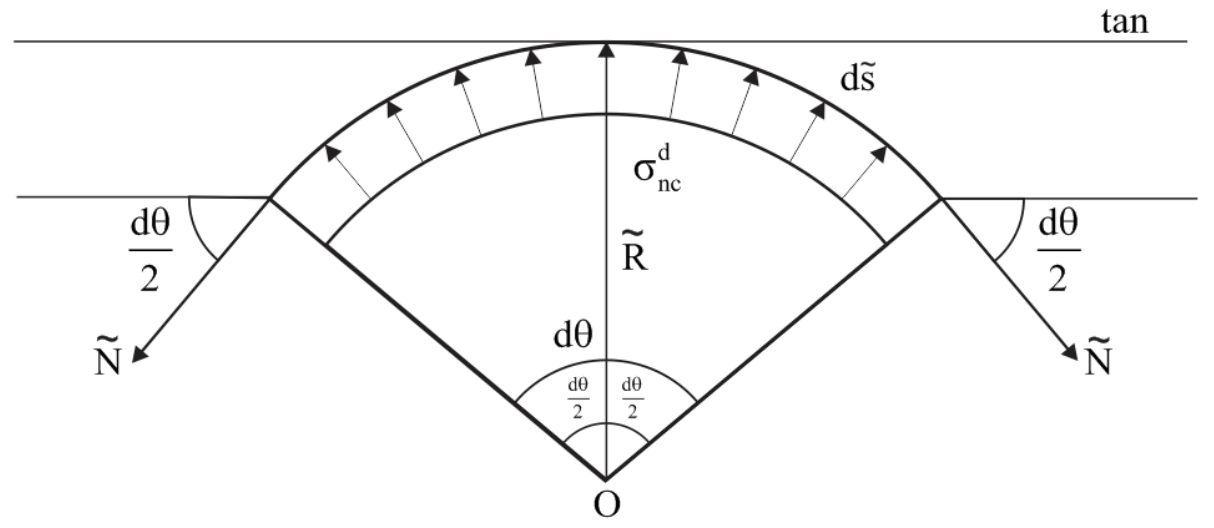

Figura 46 - Equilíbrio diferencial ortogonal à fibra. 
Para o comprimento infinitesimal $d \tilde{s}$ de fibra curva, há um centro de curvatura $O$ que define o ângulo $d \theta$ e um raio de curvatura $\tilde{R}$, a partir dos quais é possível definir a relação geométrica:

$$
d \tilde{s}=\tilde{R} d \theta
$$

Da condição de equilíbrio na direção ortogonal à fibra tem-se que:

$$
\sigma_{n c}^{d} \tilde{t} d \tilde{s}=\sigma_{n c}^{d} \tilde{t}(\tilde{R} d \theta)=2 \tilde{N} \operatorname{sen}\left(\frac{d \theta}{2}\right)=\tilde{N} d \theta
$$

$\mathrm{ou}$

$$
\sigma_{n c}^{d}(\eta)=\frac{1}{\tilde{t}} \frac{\tilde{N}(\eta)}{\tilde{R}(\eta)}
$$

Como $\tilde{N}(\eta)$ é conhecida da Eq. (5.7), é necessário calcular a curvatura $1 / \tilde{R}(\eta)$, dada por:

$$
\frac{1}{\tilde{R}(\eta)}=\frac{\frac{d^{2} \tilde{y}_{2}}{d \eta^{2}} \frac{d \tilde{y}_{1}}{d \eta}-\frac{d^{2} \tilde{y}_{1}}{d \eta^{2}} \frac{d \tilde{y}_{2}}{d \eta}}{(\tilde{J}(\eta))^{3}} .
$$

Substituindo a Eq. (5.13) na Eq. (5.12) tem-se a expressão final para a tensão normal de contato entre fibra e matriz dada por:

$$
\sigma_{n c}^{d}(\eta)=\frac{1}{\tilde{t}} \tilde{N}(\eta) \frac{\frac{d^{2} \tilde{y}_{2}}{d \eta^{2}} \frac{d \tilde{y}_{1}}{d \eta}-\frac{d^{2} \tilde{y}_{1}}{d \eta^{2}} \frac{d \tilde{y}_{2}}{d \eta}}{(\tilde{J}(\eta))^{3}}
$$

A Figura 47 mostra o aspecto geral da sub-rotina principal que gerencia a obtenção da tensão normal de contato nas fibras. 


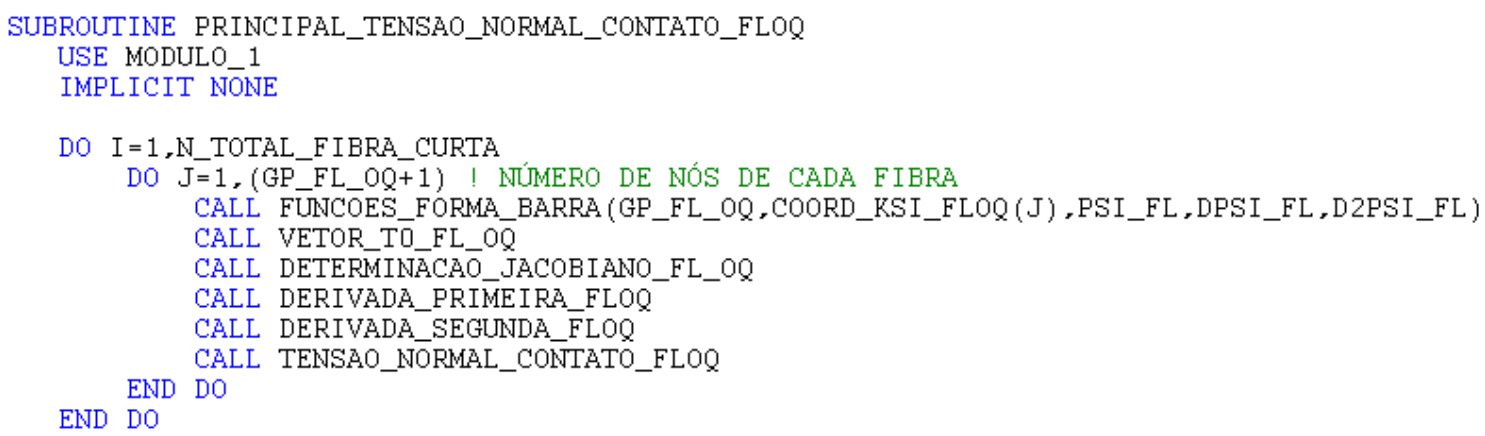

END SUBROUTINE PRINCIPAL_TENSAO_NORMAL_CONTATO_FLOQ

Figura 47 - Sub-rotina principal que gerencia a determinação da tensão normal de contato nas fibras.

\subsection{FORMULAÇÃ̃O MÉDIA}

A Figura 48 mostra a transferência de força interna $\overrightarrow{\mathrm{F}}^{\text {int }}$, da matriz para a fibra, em um nó genérico de uma fibra considerada e os vetores, normal $\vec{n}$ e tangencial $\vec{t}$, unitários calculados neste mesmo nó. As componentes, normal $\overrightarrow{\mathrm{P}}$ e tangencial $\overrightarrow{\mathrm{Q}}$, da força transferida são dadas por:

$$
\begin{aligned}
& \overrightarrow{\mathrm{Q}}=\overrightarrow{\mathrm{F}}^{\mathrm{int}} \cdot \overrightarrow{\mathrm{t}}, \\
& \overrightarrow{\mathrm{P}}=\overrightarrow{\mathrm{F}}^{\mathrm{int}} \cdot \overrightarrow{\mathrm{n}} .
\end{aligned}
$$

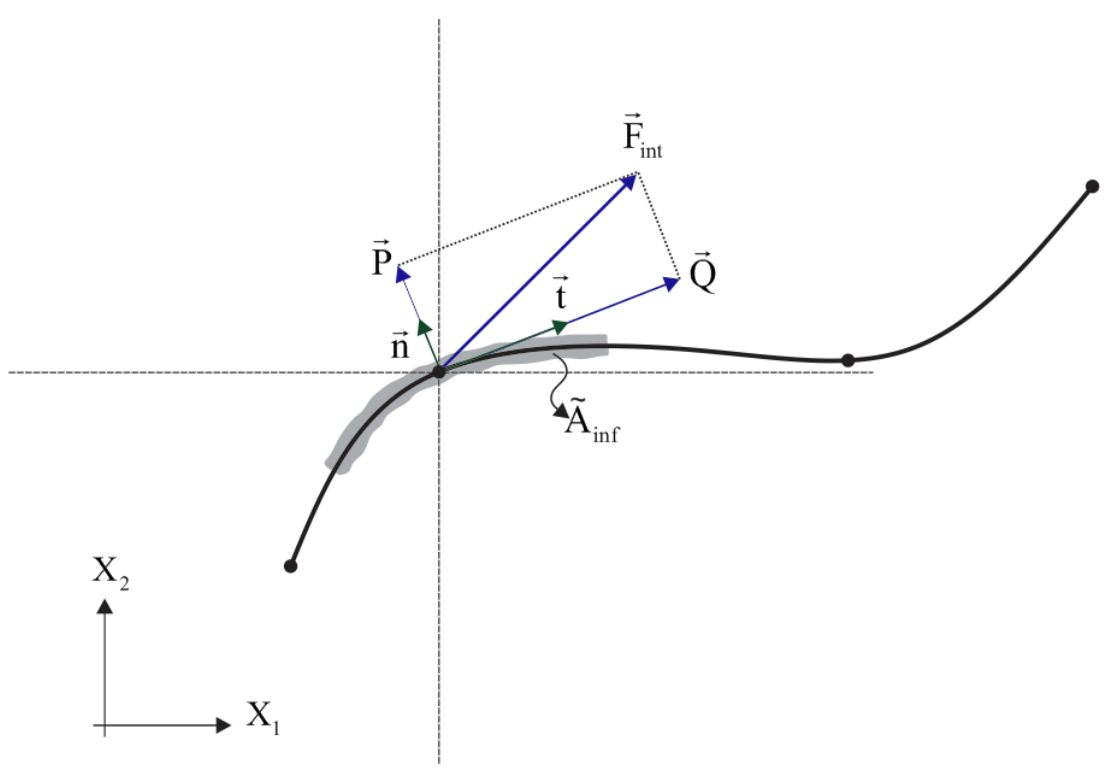

Figura 48 - Força interna transferida e área de influência. 
As forças normal e tangencial dadas pelas Eqs. (5.15) e (5.16) são divididas pela área de influência em que atuam, mostrada na Figura 48, e os valores médios obtidos são dados, respectivamente, por:

$$
\begin{aligned}
& \vec{\sigma}_{\mathrm{a}}^{\mathrm{m}}=\overrightarrow{\mathrm{F}}^{\mathrm{int}} \cdot \overrightarrow{\mathrm{t}} / \tilde{\mathrm{A}}_{\mathrm{inf}}=\overrightarrow{\mathrm{Q}} / \tilde{\mathrm{A}}_{\mathrm{inf}}, \\
& \vec{\sigma}_{\mathrm{nc}}^{\mathrm{m}}=\overrightarrow{\mathrm{F}}^{\mathrm{int}} \cdot \overrightarrow{\mathrm{n}} / \tilde{\mathrm{A}}_{\mathrm{inf}}=\overrightarrow{\mathrm{P}} / \tilde{\mathrm{A}}_{\mathrm{inf}} .
\end{aligned}
$$

Para os nós comuns a dois elementos o valor resultante é dado pela média entre os valores obtidos para o nó de cada elemento.

Diferentemente da formulação diferencial apresentada na Seção 5.2, a Eq. (5.17) calcula a tensão de aderência a partir da força total transferida para o nó. Quando o modelo permite transferência de força por tensão normal, na direção da barra, para o nó da extremidade da fibra deve-se modificar a Eq. (5.17) para:

$$
\vec{\sigma}_{\mathrm{a}}^{\mathrm{m}}=\left(\overrightarrow{\mathrm{F}}^{\mathrm{int}} \cdot \overrightarrow{\mathrm{t}}-\tilde{\mathrm{N}}\right) / \tilde{\mathrm{A}}_{\mathrm{inf}}
$$

A Figura 49 mostra o aspecto geral da sub-rotina principal que gerencia a obtenção da tensão normal de contato e tensão de aderência nas fibras pela formulação média.

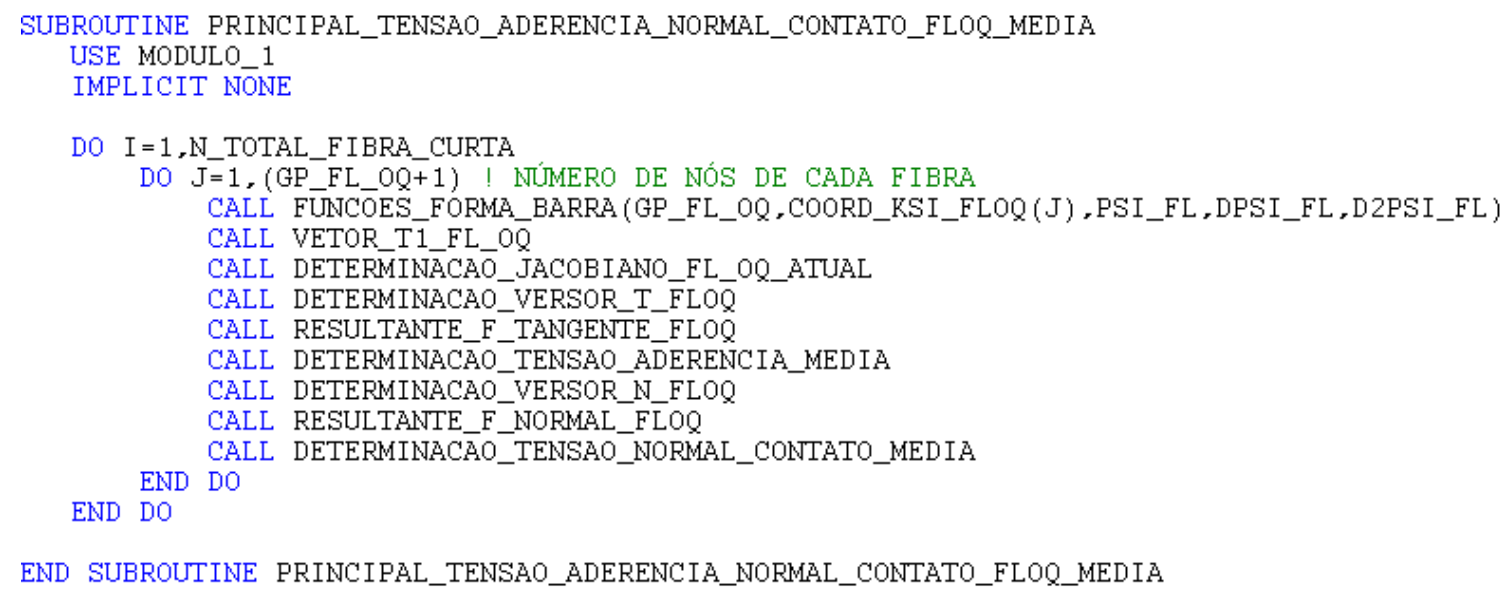

END SUBROUTINE PRINCIPAL_TENSAO_ADERENCIA_NORMAL_CONTATO_FLOQ_MEDIA

Figura 49 - Sub-rotina principal que gerencia a obtenção da tensão normal de contato e tensão de aderência nas fibras pela formulação média. 


\subsection{EXEMPLOS NUMÉRICOS}

Para avaliar o comportamento das formulações apresentadas, dois exemplos são analisados. O primeiro consiste em um domínio retangular reforçado com quatro enrijecedores retos e o segundo um domínio retangular reforçado com fibras com trechos curvos.

\subsubsection{EXEMPLO1: BARRA REFORÇADA COM FIBRAS RETAS}

Neste exemplo, um domínio retangular reforçado por quarto enrijecedores igualmente espaçados foi analisado (Figura 50). A matriz tem $L_{m}=20 \mathrm{~cm}$ de comprimento, $h_{m}=2 \mathrm{~cm}$ de altura e $b_{m}=1 \mathrm{~cm}$ de espessura. O comprimento das fibras é $L_{f}=19,8 \mathrm{~cm}$. O módulo de elasticidade e o coeficiente de Poisson adotados para a matriz são dados, respectivamente, por $E_{m}=2,0 \mathrm{MN} / \mathrm{cm}^{2}$ e $v_{m}=0,0$. Cada fibra possui módulo de elasticidade $E_{f}=100,0 \mathrm{MN} / \mathrm{cm}^{2}$, área da seção transversal $A_{f}=0,01 \mathrm{~cm}^{2}$ e a mesma espessura da matriz. Devido à dupla simetria somente um quarto do problema foi discretizado. O problema foi resolvido por controle de deslocamentos sendo $\Delta=0,5 \mathrm{~cm}$ o deslocamento total imposto na extremidade da barra. Para a análise linear do problema o deslocamento imposto foi dividido por $10^{4}$ e os resultados obtidos foram multiplicados por $10^{4}$. Para pequenos deslocamentos a Lei de Saint-Venant-Kirchhoff se aproxima da Lei de Hooke.
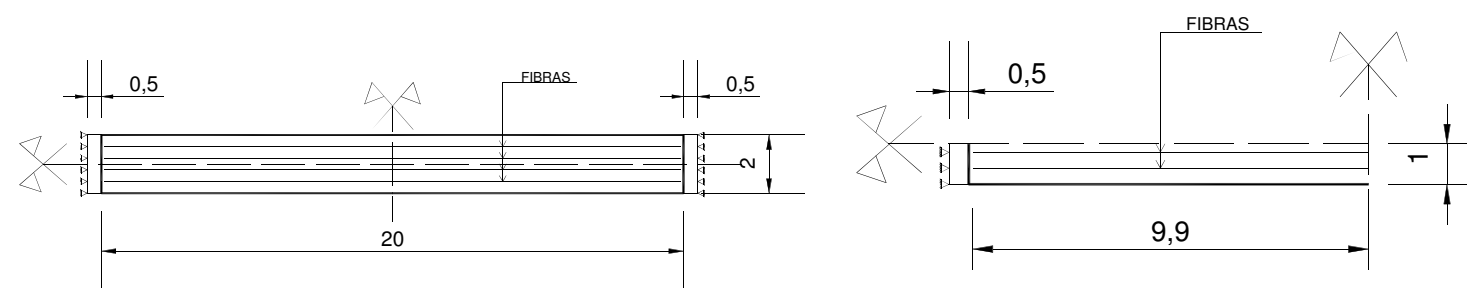

Figura 50 - Barra reforçada submetida a deslocamento controlado (unidades em $\mathrm{cm}$ ).

Para todas as análises realizadas foram utilizados 864 elementos finitos triangulares com aproximação cúbica para as variáveis para discretizar a matriz. Os elementos foram dispostos de duas maneiras: (a) igualmente espaçados resultando em uma malha como 
mostrado na Figura 51 e (b) distorcidos resultando em uma malha como mostrado na Figura 54. Para discretizar as fibras, foram utilizados oitenta elementos finitos com aproximação cúbica para as variáveis.

A Figura 52 mostra a distribuição da força normal e da tensão de aderência na fibra superior obtidas com a formulação diferencial para a malha mostrada na Figura 51. Para a tensão de aderência dois casos foram testados. No primeiro, a força normal e as posições possuem a mesma ordem de aproximação e no segundo caso a aproximação da força normal é uma ordem menor que a aproximação adotada para as posições. A Figura 53 mostra a tensão de aderência obtida com a formulação média para a malha da Figura 51.

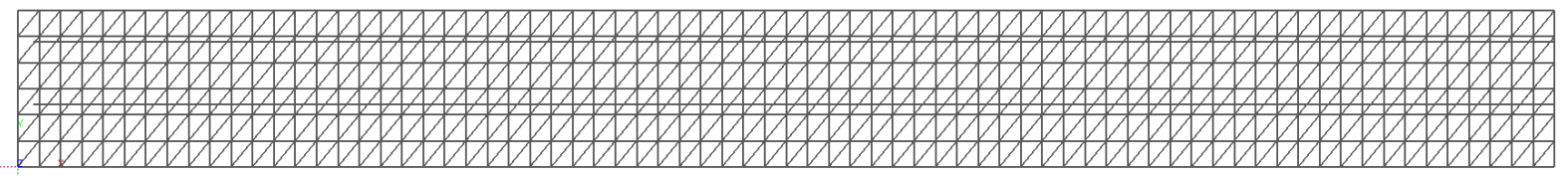

Figura 51 - Malha igualmente espaçada: 864 elementos triangulares e 80 elementos de fibra - 8264 graus de liberdade.
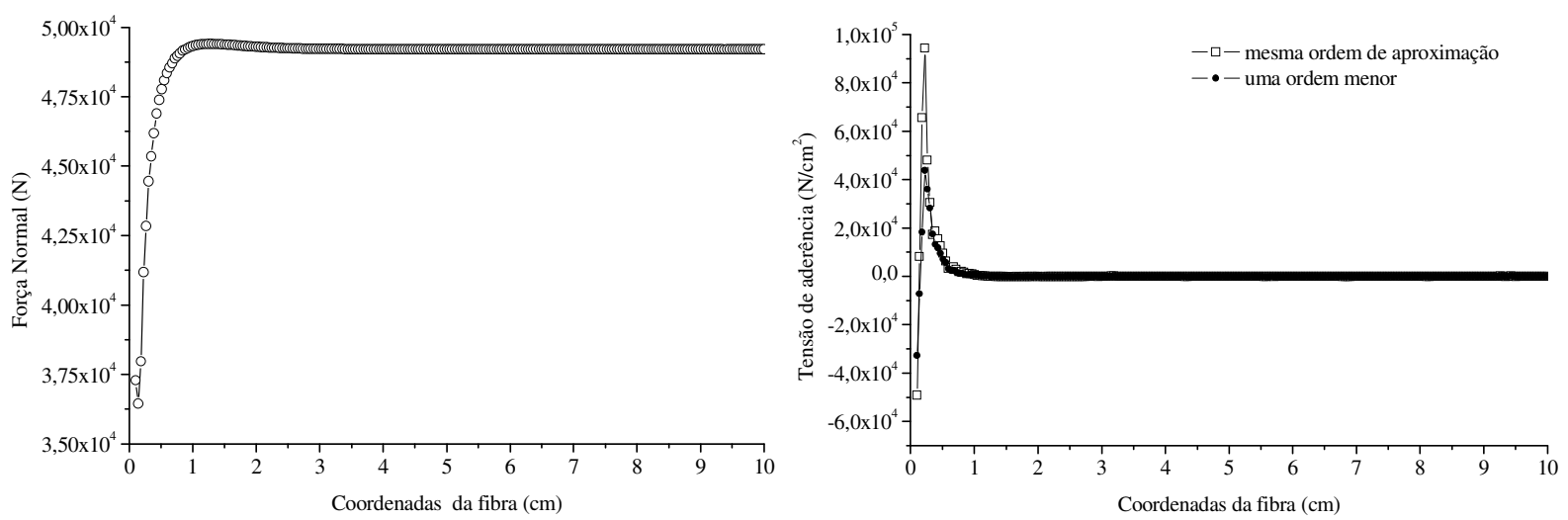

Figura 52 - Força normal e tensão de aderência: formulação diferencial.

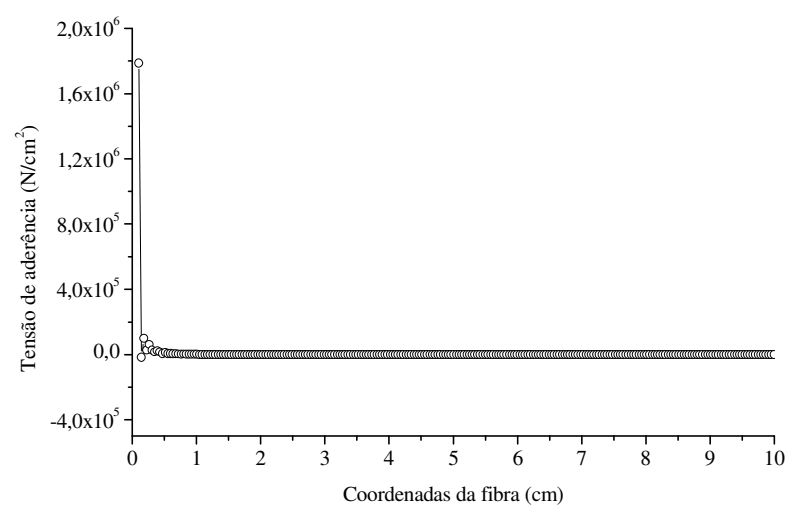

Figura 53 - Tensão de aderência: formulação média.

A partir da Figura 52 e da Figura 53 é possível observar a presença de oscilações nos 
resultados obtidos em uma região próxima à extremidade da fibra para todos os casos analisados. Acredita-se que estas oscilações ocorrem, pois a malha utilizada não é refinada o suficiente para representar o problema de forma apropriada. Para melhorar os resultados obtidos, a malha inicialmente empregada, Figura 51, foi melhorada impondo-se uma distorção nas posições dos nós de canto dos elementos triangulares na forma $X_{i}=X_{i}\left(X_{I} / L\right)^{3}$. Os nós internos de cada elemento permaneceram igualmente espaçados e a malha resultante pode ser observada na Figura 54. O número de elementos permaneceu inalterado.

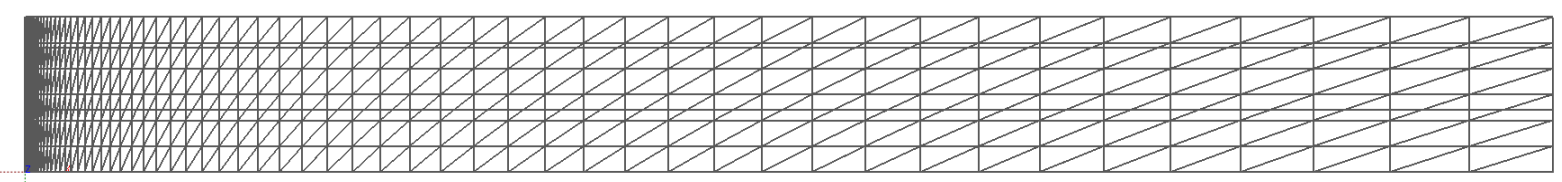

Figura 54 - Malha distorcida: 864 elementos triangulares e 80 elementos de fibra - 8246 graus de liberdade.
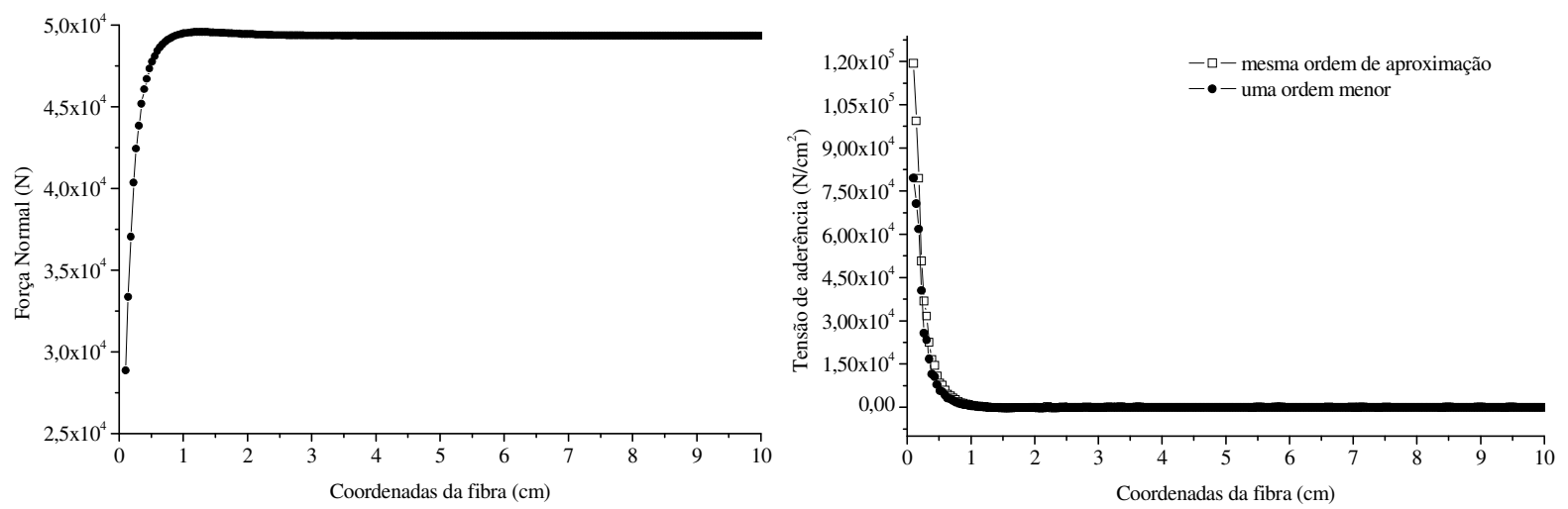

Figura 55 - Força normal na fibra e tensão de aderência: formulação diferencial.

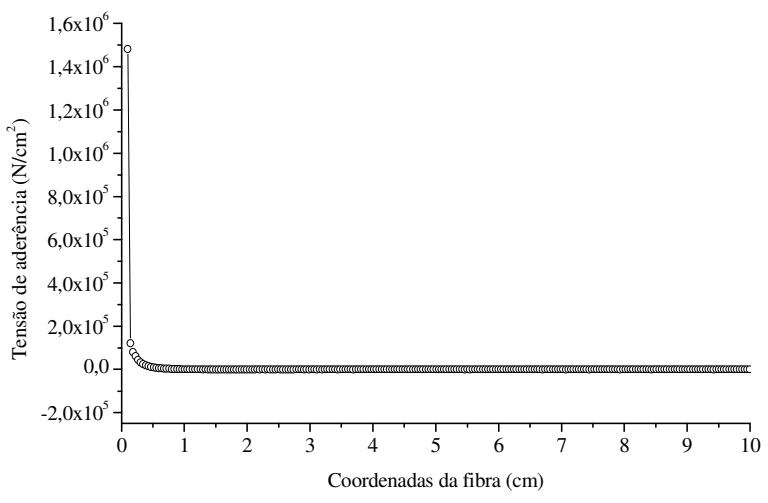

Figura 56 - Tensão de aderência: formulação média.

A Figura 55 mostra a distribuição da força normal e da tensão de aderência na fibra superior obtidas com a formulação diferencial para a malha distorcida mostrada na Figura 54. 
Para a tensão de aderência dois casos foram testados. No primeiro a força normal e as posições possuem a mesma ordem de aproximação e no segundo caso a aproximação da força normal é uma ordem menor que a aproximação adotada para as posições. A Figura 56 mostra a tensão de aderência obtida com a formulação média para a malha distorcida da Figura 54.

A partir dos resultados obtidos é possível observar que a malha mais refinada na região de concentração de tensão regularizou o comportamento observado nas curvas de força normal e tensão de aderência em todos os casos considerados.

\subsubsection{EXEMPLO 2: BARRA REFORÇADA COM FIBRAS CURVAS}

Este exemplo consiste em um ensaio de tração de uma chapa reforçada com fibras conforme mostrado na Figura 57. As fibras possuem trechos curvos nas extremidades com raio $R=0,5 \mathrm{~cm}$. O módulo de elasticidade e o coeficiente de Poisson adotados para a matriz são dados, respectivamente, por $E_{m}=100 \mathrm{kN} / \mathrm{cm}^{2} E_{m}=100 \mathrm{kN} / \mathrm{cm}^{2}$ e $v_{m}=0,0$. Para a fibra, o módulo de elasticidade e a área da seção transversal são dados, respectivamente, por $E_{f}=2 M N / \mathrm{cm}^{2}$ e $A_{f}=0,1 \mathrm{~cm}^{2}$. Um deslocamento total de $u=0,1 \mathrm{~cm}$ foi aplicado nas extremidades da chapa.
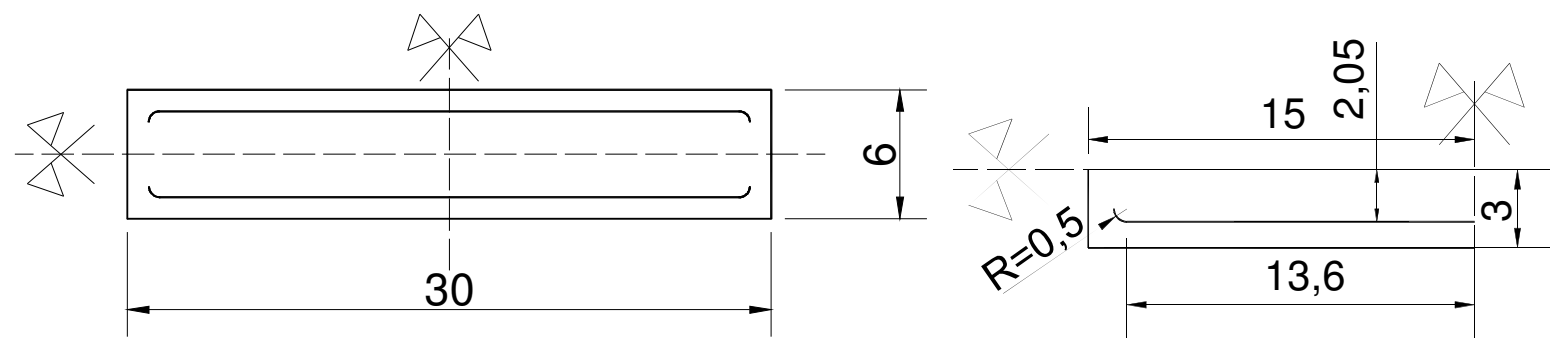

Figura 57 - Barra reforçada com fibras curvas (unidades em $\mathrm{cm}$ ).

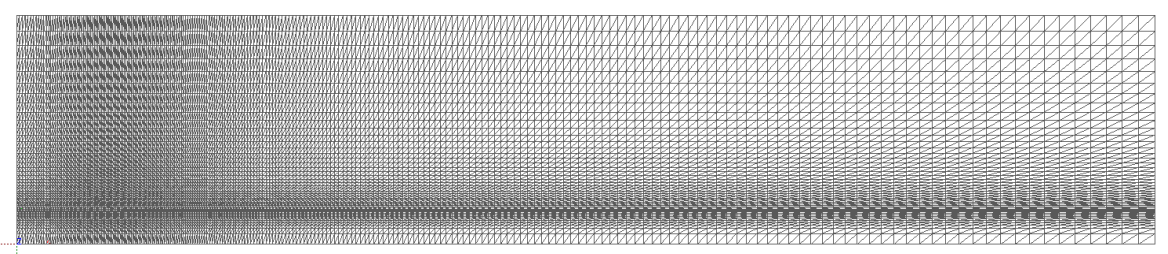

Figura 58 - Malha distorcida: 225x45 elementos triangulares cúbicos - 183872 graus de liberdade.

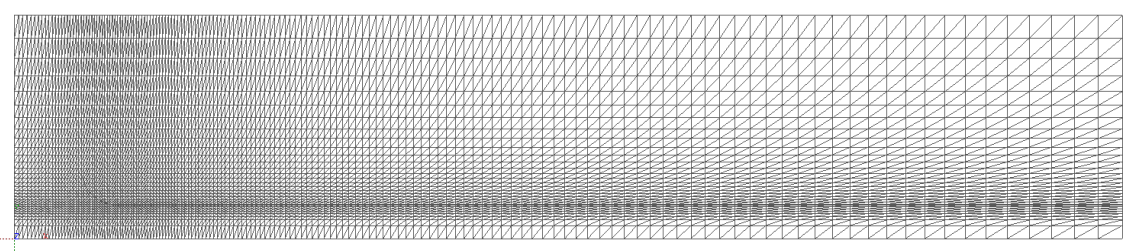

Figura 59 - Malha distorcida: 150x30 elementos triangulares cúbicos - 82082 graus de liberdade. 


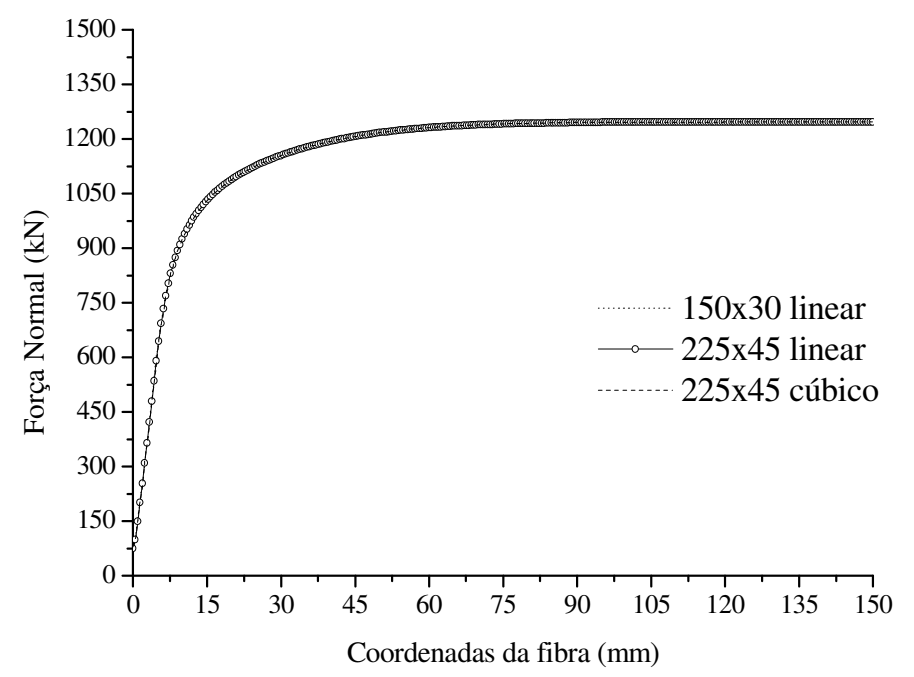

Figura 60 - Força normal - kN.
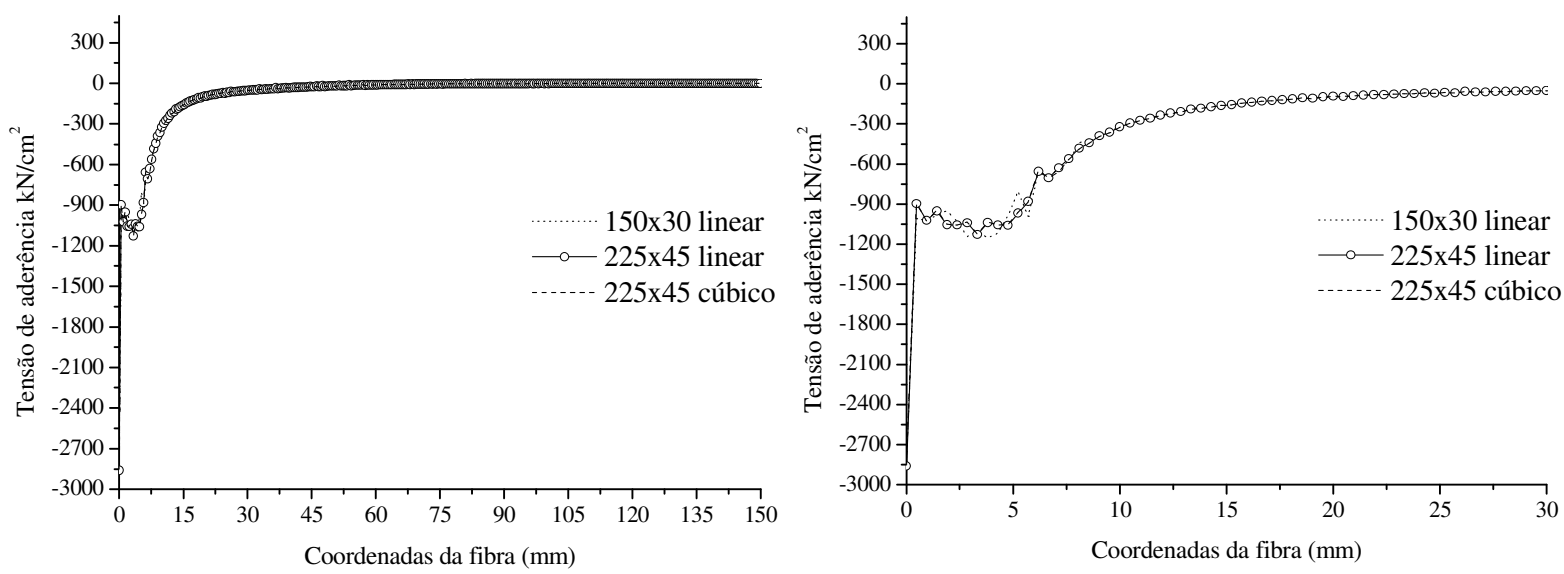

Figura 61 - Tensão de aderência $-\mathrm{kN} / \mathrm{cm}^{2}$.
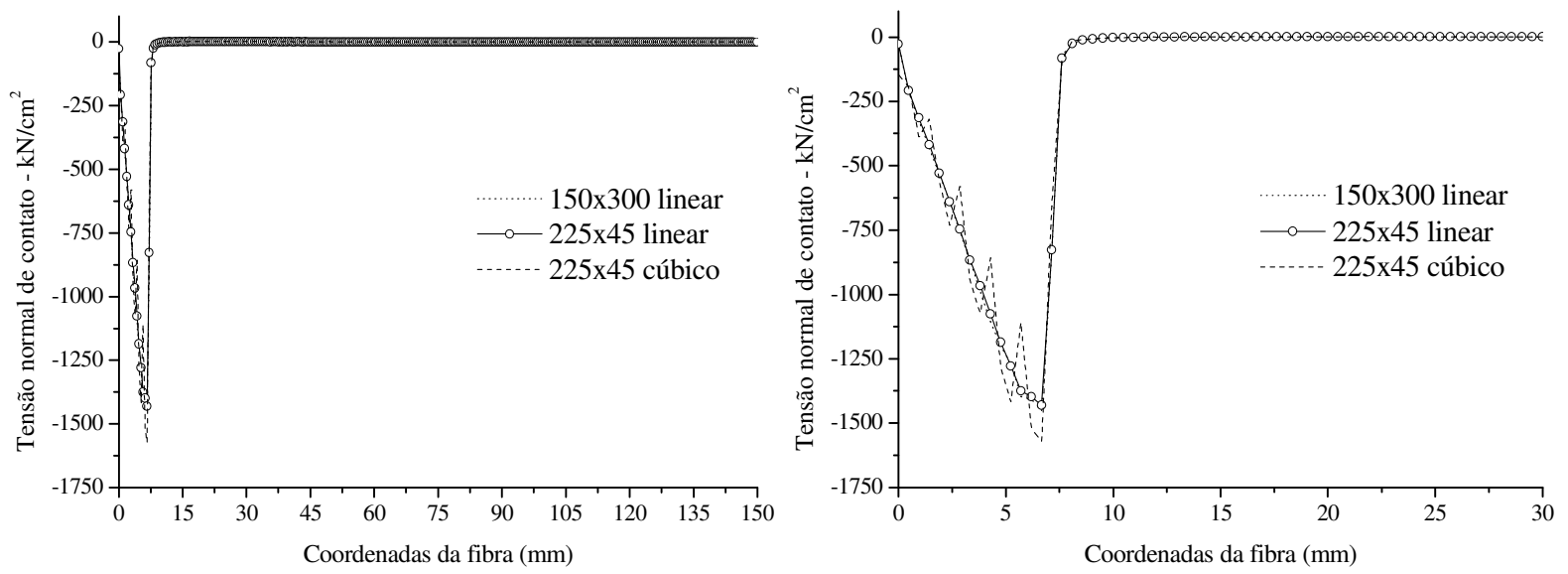

Figura 62 - Tensão Normal de contato $-\mathrm{kN} / \mathrm{cm}^{2}$.

Devido à dupla simetria somente um quarto do problema foi discretizado. Para as análises utilizaram-se duas malhas para discretizar a matriz. A primeira com $225 \times 45$ 
elementos cúbicos triangulares e outra com 150x30 elementos cúbicos triangulares, mostradas na Figura 58 e na Figura 59, respectivamente. A fibra foi discretizada com 315 elementos lineares e 105 elementos cúbicos igualmente espaçados.

A Figura 60 mostra a distribuição da força normal na fibra medida a partir da extremidade curva do enrijecedor. A tensão de aderência e a tensão normal de contato, juntamente com uma ampliação da região próxima ao gancho, são mostradas na Figura 61 e na Figura 62, respectivamente.

Embora o comportamento da distribuição esteja conforme esperado é possível observar a presença de oscilações nas tensões obtidas, principalmente para o elemento cúbico.

Acredita-se que uma possível explicação para a presença das oscilações está associada à hipótese de contato-perfeito adotada para modelar fibra-matriz. Como este problema não foi solucionado no presente trabalho, sugere-se para trabalhos futuros testar outras estratégias, como por exemplo, considerar modelos de deslizamento fibra-matriz para tentar eliminar as oscilações observadas. 


\section{CASCAS LAMINADAS REFORÇADAS COM FIBRAS}

\subsection{CONSIDERAÇÕES INICIAIS}

Os métodos existentes para a análise de estruturas laminadas reforçadas por fibras podem ser organizados em pelos menos quatro grandes grupos, a saber: os modelos em que o meio laminado heterogêneo é substituído por um homogêneo equivalente; os modelos em que tanto a matriz quanto a fibra são discretizadas com elementos sólidos tridimensionais; os modelos em que a matriz é discretizada com elementos de casca e as fibras com elemento de barra e os modelos em que a rigidez da fibra é distribuída na rigidez do elemento finito de casca (BARUT et al., 2000). Enquanto o primeiro modelo dificulta a identificação da distribuição das tensões de contato fibra-matriz, o segundo e o terceiro aumentam o número de graus de liberdade do sistema de equações resultante e juntamente com o quarto exigem a coincidência dos nós da fibra com os nós da matriz na discretização do problema.

Desta forma, apresenta-se, neste capítulo, uma formulação para a introdução de fibras curvas, longas ou curtas aleatórias, nas diversas camadas que compõem um laminado, sem aumentar o número de graus de liberdade do sistema de equações resultante e sem a necessidade de coincidência de nós na discretização das fibras e da matriz.

Primeiramente, descreve-se o elemento finito de casca homogêneo não linear geométrico conforme apresentado por Coda e Paccola (2008). Este elemento possui sete parâmetros por nó, sendo três translações, três componentes do vetor generalizado e a taxa de variação da deformação ao longo da espessura, responsável por evitar a ocorrência do fenômeno de travamento volumétrico neste elemento.

Em seguida, a generalização da cinemática deste elemento para obtenção do elemento finito de casca laminada é apresentada (CODA; PACCOLA; SAMPAIO, 2013). Uma importante característica deste elemento é que não há acréscimo de graus de liberdade na discretização do problema em função do número de lâminas que compõe o laminado 
considerado.

Finalmente, a estratégia cinemática adotada para inserir as fibras em qualquer camada do laminado sem aumentar o número de graus de liberdade do problema e os exemplos de validação da formulação são apresentados.

Para fazer distinção entre os diferentes meios considerados nos equacionamentos, adota-se o padrão simbólico $(\bullet)$ para as variáveis e grandezas relacionadas ao elemento finito de casca e $(\tilde{\bullet})$ para as variáveis e grandezas relacionadas ao elemento finito de fibra curvo de ordem qualquer.

\subsection{CINEMÁTICA DO ELEMENTO FINITO DE CASCA HOMOGÊNEO}

Seja um elemento finito triangular curvo de casca com aproximação cúbica para as variáveis e espessura $\widehat{h}_{0}$ constante na configuração inicial conforme mostrado na Figura 63. As funções de forma deste elemento e suas respectivas derivadas em relação aos parâmetros nodais são apresentadas no ANEXO D.

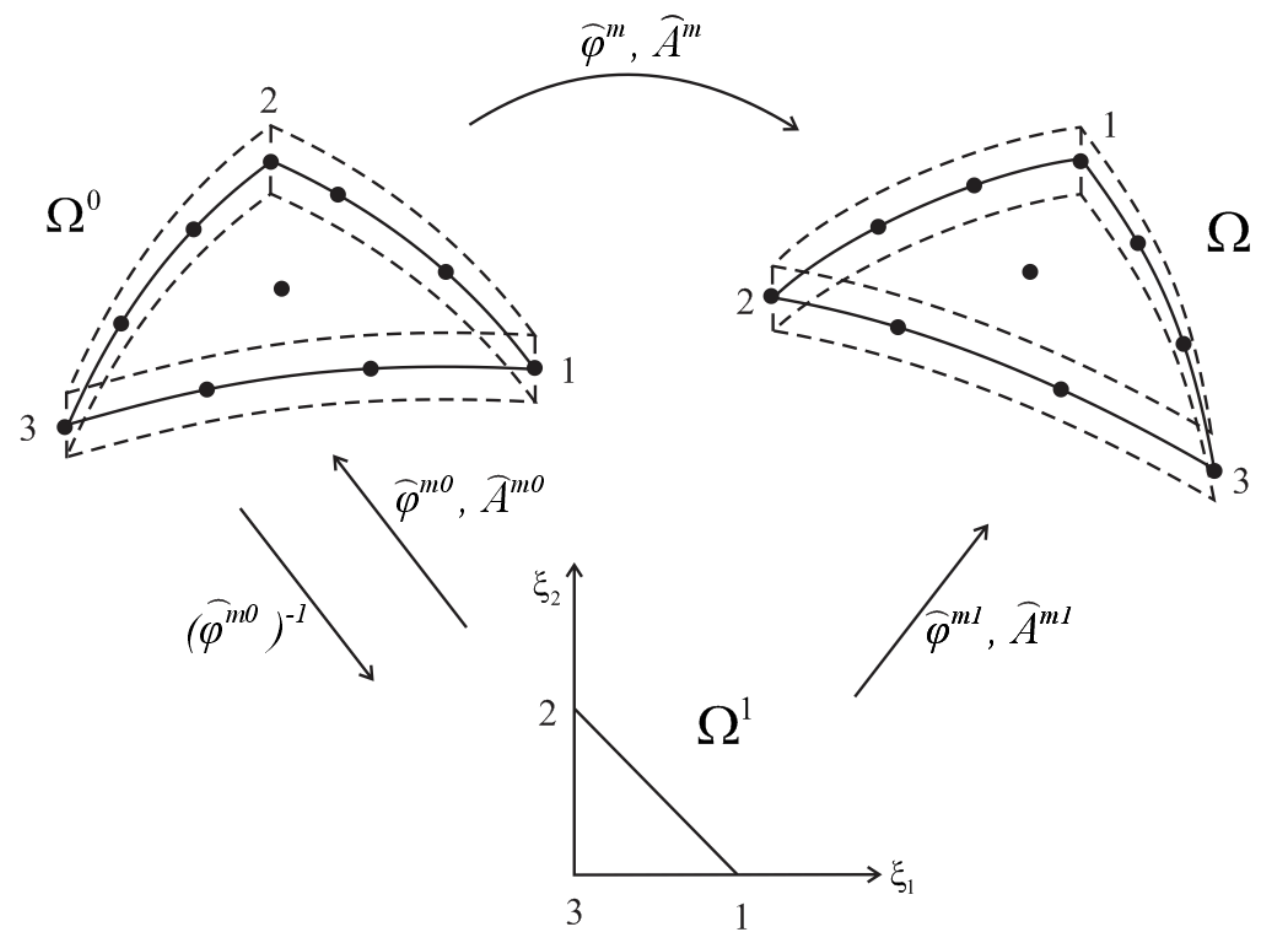

Figura 63 - Mapeamento da superfície média do elemento finito de casca. Fonte: (CODA; PACCOLA, 2008). 
Sejam $\hat{x}_{i}^{m}$ e $\hat{y}_{i}^{m}$ as coordenadas de um ponto qualquer sobre a superfície média do elemento finito de casca na configuração inicial e corrente, respectivamente e $\hat{X}_{l i}$ e $\hat{Y}_{l i}$ os valores das coordenadas nodais relacionadas a cada uma das configurações.

$\mathrm{O}$ mapeamento $\hat{\varphi}_{i}^{m 0}$ que leva do sistema auxiliar adimensional para superfície de referência na configuração inicial e o mapeamento $\widehat{\varphi}_{i}^{m 1}$ que leva do sistema auxiliar para superfície de referência na configuração corrente são dados, respectivamente, por

$$
\begin{gathered}
\hat{\varphi}_{i}^{m 0}=\widehat{x}_{i}^{m}\left(\xi_{1}, \xi_{2}, \widehat{X}_{l i}\right)=\widehat{\phi}_{l}\left(\xi_{1}, \xi_{2}\right) \widehat{X}_{l i} \\
\widehat{\varphi}_{i}^{m 1}=\widehat{y}_{i}^{m}\left(\xi_{1}, \xi_{2}, \widehat{Y}_{l i}\right)=\widehat{\phi}_{l}\left(\xi_{1}, \xi_{2}\right) \widehat{Y}_{l i} .
\end{gathered}
$$

Na Figura 63, $\widehat{\varphi}^{m}$ é o mapeamento da superfície de referência da configuração inicial para a configuração corrente, que pode ser obtido por meio de uma composição entre os mapeamentos $\hat{\varphi}_{i}^{m 0}$ e $\widehat{\varphi}_{i}^{m 1}$, sendo $\widehat{A}^{m}, \widehat{A}^{m 0}$ e $\widehat{A}^{m 1}$ os correspondentes gradientes destes mapeamentos.

Para mapear os pontos que estão fora da superfície média ou de referência do elemento finito de casca adota-se um vetor de posicionamento $\widehat{\vec{g}}^{0}$ associado à configuração inicial do corpo e um vetor de posicionamento $\widehat{\vec{g}}^{1}$ associado à configuração atual do corpo, conforme ilustrado na Figura 64.
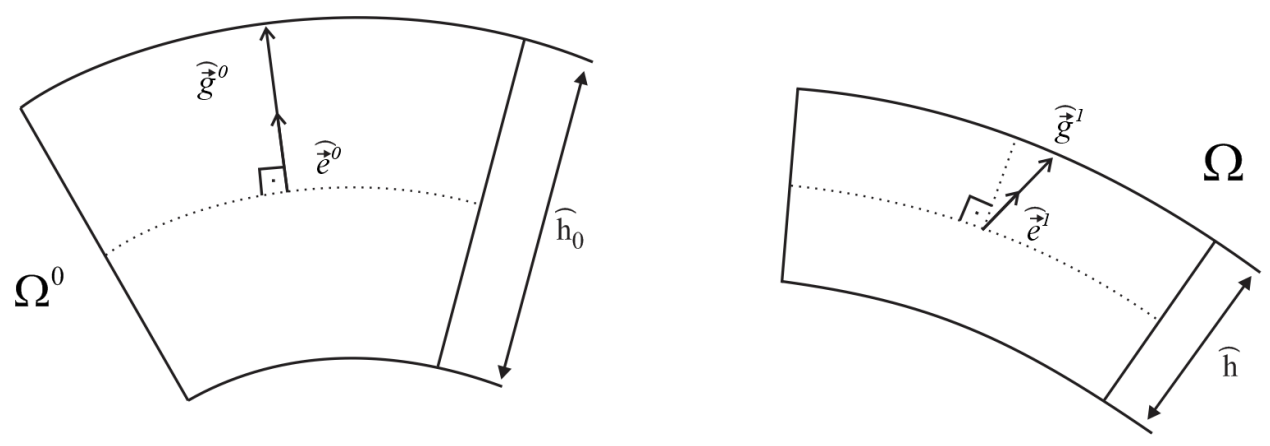

Figura 64 - Mapeamento dos pontos fora da superfície média. Fonte: (PASCON, 2008).

Observa-se na Figura 64 que a espessura da casca, inicialmente constante, $\hat{h}_{0}$, passa a ser $\widehat{h}$ na configuração atual. Essa variação da espessura é considerada através da mudança de 
tamanho do vetor $\widehat{\vec{g}}^{1}$, que pode alongar ou diminuir dependendo do processo de mudança de configuração.

Os vetores $\widehat{\vec{g}}^{0}$ e $\widehat{\vec{g}}^{1}$ representam a diferença entre as coordenadas de um ponto fora da superfície média e seu correspondente, pertencente à superfície média.

Por outro lado, com os vetores $\hat{\vec{g}}^{0}$ e $\widehat{\vec{g}}^{1}$, os pontos genéricos $\hat{x}_{i}$ e $\hat{y}_{i}$ no domínio do elemento finito, na configuração inicial e corrente, respectivamente, podem ser obtidos somando-se a estes vetores os mapeamentos da superfície de referência $\hat{x}_{i}^{m}$ e $\hat{y}_{i}^{m}$, pelas expressões:

$$
\begin{aligned}
& \hat{x}_{i}=\hat{x}_{i}^{m}+\hat{g}_{i}^{0}, \\
& \hat{y}_{i}=\hat{y}_{i}^{m}+\hat{g}_{i}^{1} .
\end{aligned}
$$

A Figura 65 ilustra o mapeamento dos pontos fora da superfície média em ambas as configurações, inicial e corrente.

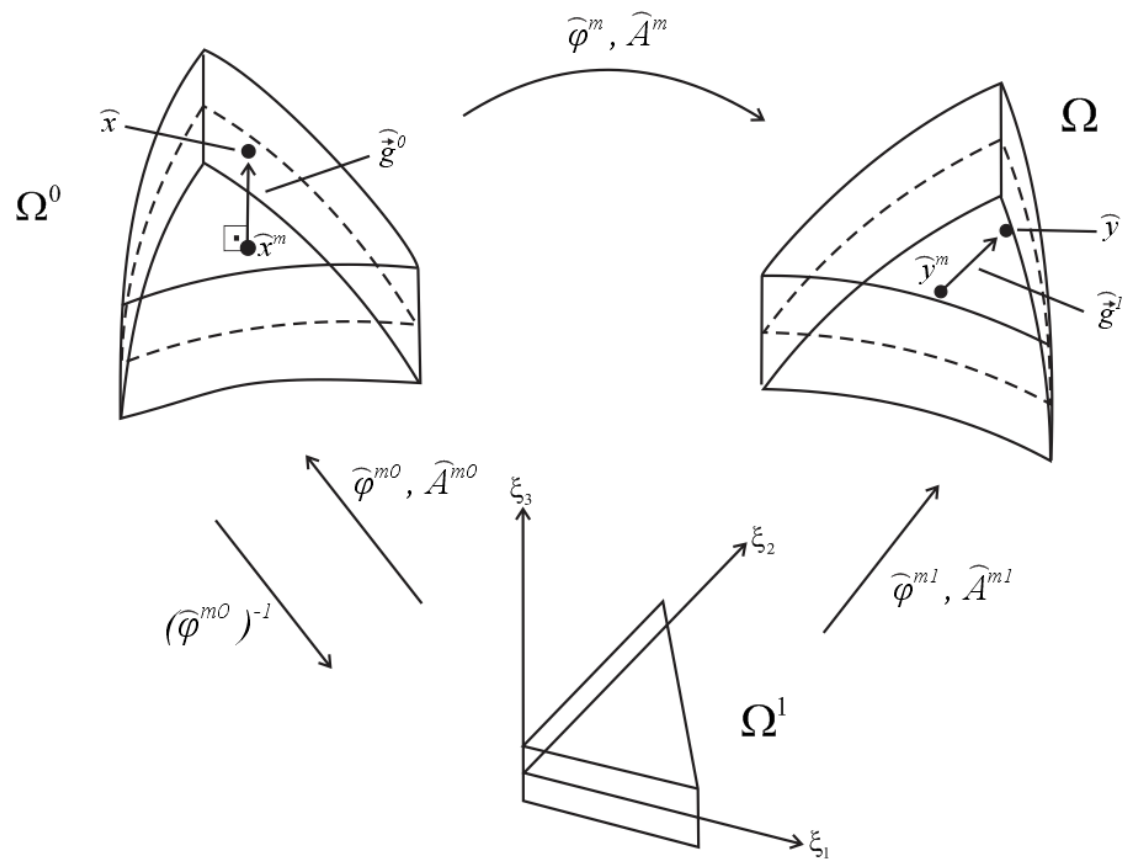

Figura 65 - Mapeamento dos pontos fora da superfície média do elemento de casca. Fonte: (CODA; PACCOLA, 2008).

Considerando-se deformação constante ao longo da espessura da casca, os vetores $\widehat{\vec{g}}^{0}$ 
e $\widehat{\vec{g}}^{1}$ podem ser escritos em função das variáveis adimensionais, nas configurações, inicial e atual, respectivamente, como:

$$
\begin{gathered}
\hat{g}_{i}^{0}=\frac{\widehat{h}_{0}}{2} \widehat{e}_{i}^{0}\left(\xi_{1}, \xi_{2}\right) \xi_{3}, \\
\hat{g}_{i}^{1}=\frac{\widehat{h}\left(\xi_{1}, \xi_{2}\right)}{2} \hat{e}_{i}^{1}\left(\xi_{1}, \xi_{2}\right) \xi_{3},
\end{gathered}
$$

sendo $\widehat{h}_{0}$ a espessura inicial do elemento de casca, $\hat{h}$ a espessura atual, $\widehat{\vec{e}}^{0}$ um vetor unitário normal à superfície média inicial e $\widehat{\vec{e}}^{1}$ um vetor unitário, porém não necessariamente normal à superfície média da casca na configuração atual.

Da forma como descrito até então, este elemento, apresenta o fenômeno indesejado do travamento volumétrico. Para evitá-lo, adiciona-se à cinemática do problema, a variável escalar, $a$, que considera a taxa de variação da espessura ao longo do elemento de casca e permite reescrever a Eq. (6.6) como:

$$
\hat{g}_{i}^{1}=\frac{\widehat{h}\left(\xi_{1}, \xi_{2}\right)}{2} \hat{e}_{i}^{1}\left(\xi_{1}, \xi_{2}\right)\left[\xi_{3}+a\left(\xi_{1}, \xi_{2}\right) \xi_{3}^{2}\right] .
$$

Admitindo-se que a espessura da casca na configuração inicial é constante, o vetor $\widehat{\vec{e}}^{0}$ na Eq. (6.5) é aproximado a partir de vetores normais unitários, com componentes $\widehat{N}_{i l}^{0}$ avaliadas nos pontos nodais $l$ da configuração inicial, pela expressão:

$$
\widehat{e}_{i}^{0}\left(\xi_{1}, \xi_{2}\right)=\widehat{\phi}_{l}\left(\xi_{1}, \xi_{2}\right) \widehat{N}_{i l}^{0}
$$

Para a configuração atual, Coda e Paccola (2008) propõem a aproximação:

$$
\widehat{h}\left(\xi_{1}, \xi_{2}\right) \hat{e}_{i}^{1}\left(\xi_{1}, \xi_{2}\right)=\widehat{h}_{0} \widehat{g}_{i}\left(\xi_{1}, \xi_{2}\right)
$$

onde $\widehat{g}_{i}\left(\xi_{1}, \xi_{2}\right)$ é vetor generalizado não unitário e não ortogonal à superfície média da casca na configuração atual. 
A partir da Eq. (6.9), a variação da espessura da casca ao longo do elemento pode ser escrita como:

$$
\widehat{h}\left(\xi_{1}, \xi_{1}\right)=\widehat{h}_{0} \sqrt{\widehat{g}_{i}\left(\xi_{1}, \xi_{1}\right) \hat{g}_{i}\left(\xi_{1}, \xi_{1}\right)}
$$

sendo as componentes do vetor unitário $\widehat{\vec{e}}^{1}$ dadas por:

$$
\hat{e}_{i}^{1}=\frac{\widehat{g}_{i}\left(\xi_{1}, \xi_{1}\right)}{\sqrt{\hat{g}_{i}\left(\xi_{1}, \xi_{1}\right) \hat{g}_{i}\left(\xi_{1}, \xi_{1}\right)}} .
$$

Finalmente, as componentes dos vetores $\overline{\vec{g}}^{0}$ e $\widehat{\vec{g}}^{1}$ podem ser reescritas como:

$$
\begin{gathered}
\hat{g}_{i}^{0}=\frac{\widehat{h}_{0}}{2} \xi_{3} \widehat{\phi}_{l}\left(\xi_{1}, \xi_{2}\right) \widehat{N}_{i l}^{0}, \\
\hat{g}_{i}^{1}=\frac{\widehat{h}_{0}}{2}\left[\xi_{3}+a\left(\xi_{1}, \xi_{2}\right) \xi_{3}^{2}\right] \widehat{\phi}_{l}\left(\xi_{1}, \xi_{2}\right) \widehat{G}_{i l},
\end{gathered}
$$

onde $\widehat{G}_{l l}$ são incógnitas nodais do vetor generalizado na configuração corrente.

A partir das Eqs. (6.3)-(6.4) e Eqs. (6.12)-(6.13), tem-se que os mapeamentos do elemento finito de casca para as configurações, inicial e atual, são dados respectivamente por:

$$
\begin{gathered}
\widehat{\varphi}_{i}^{0}=\widehat{x}_{i}=\widehat{\phi}_{l}\left(\xi_{1}, \xi_{2}\right) \widehat{X}_{l i}+\frac{\widehat{h}_{0}}{2} \xi_{3} \widehat{\phi}_{l}\left(\xi_{1}, \xi_{2}\right) \widehat{N}_{i l}^{0}, \\
\hat{\varphi}_{i}^{1}=\widehat{y}_{i}=\widehat{\phi}_{l}\left(\xi_{1}, \xi_{2}\right) \widehat{Y}_{l i}+\frac{\widehat{h}_{0}}{2}\left[\xi_{3}+a\left(\xi_{1}, \xi_{2}\right) \xi_{3}^{2}\right] \widehat{\phi}_{l}\left(\xi_{1}, \xi_{2}\right) \widehat{G}_{i l},
\end{gathered}
$$

sendo $a\left(\xi_{1}, \xi_{2}\right)=\widehat{\phi}_{l}\left(\xi_{1}, \xi_{2}\right) \widehat{a}_{l}$ a aproximação para a variação linear da deformação ao longo da espessura e $\widehat{a}_{l}$ os seus valores nodais.

Os gradientes da função mudança de configuração $\widehat{A}_{i j}^{1}$, ou seja, as derivadas das funções $\widehat{\varphi}_{i}^{\prime}$ em relação às variáveis adimensionais são dadas por: 


$$
\begin{gathered}
\widehat{\mathrm{A}}_{i 1}^{1}=\widehat{\phi}_{l, 1}\left(\xi_{1}, \xi_{2}\right) \widehat{Y}_{l i}+\frac{\widehat{h}_{0}}{2}\left\{\left[\xi_{3}+\widehat{\phi}_{l}\left(\xi_{1}, \xi_{2}\right) \widehat{a}_{l} \xi_{3}^{2}\right] \widehat{\phi}_{l, 1}\left(\xi_{1}, \xi_{2}\right) \widehat{G}_{i l}+\left[\widehat{\phi}_{l, 1}\left(\xi_{1}, \xi_{2}\right) \widehat{a}_{l} \xi_{3}^{2}\right] \widehat{\phi}_{l}\left(\xi_{1}, \xi_{2}\right) \widehat{G}_{i l}\right\}, \\
\widehat{\mathrm{A}}_{i 2}^{1}=\widehat{\phi}_{l, 2}\left(\xi_{1}, \xi_{2}\right) \widehat{Y}_{l i}+\frac{\widehat{h}_{0}}{2}\left\{\left[\xi_{3}+\widehat{\phi}_{l}\left(\xi_{1}, \xi_{2}\right) \widehat{a}_{l} \xi_{3}^{2}\right] \widehat{\phi}_{l, 2}\left(\xi_{1}, \xi_{2}\right) \widehat{G}_{i l}+\left[\widehat{\phi}_{l, 2}\left(\xi_{1}, \xi_{2}\right) \widehat{a}_{l} \xi_{3}^{2}\right] \widehat{\phi}_{l}\left(\xi_{1}, \xi_{2}\right) \widehat{G}_{i l}\right\}, \\
\tilde{\mathrm{A}}_{i 3}^{1}=\frac{\widehat{h}_{0}}{2}\left[1+2 \widehat{\phi}_{l}\left(\xi_{1}, \xi_{2}\right) \widehat{\mathrm{a}}_{l} \xi_{3}\right] \widehat{\phi}_{l}\left(\xi_{1}, \xi_{2}\right) \widehat{G}_{i l} .
\end{gathered}
$$

com $i=1, . ., 3$.

Utilizando a Lei Constitutiva de Saint-Venant-Kirchhoff para obter a energia específica de deformação, a energia potencial total do sistema, para um volume de referência $\widehat{V}_{0}$, é dada por:

$$
\widehat{\Pi}=\int_{V_{0}} \frac{1}{2} \widehat{E}_{k l} \widehat{C}_{k l i j} \widehat{E}_{i j} d \widehat{V}_{0}-\widehat{F}_{k} \widehat{Y}_{k}
$$

Aplicando-se o Princípio da Mínima Energia Potencial Total à Eq. (6.19), tem-se que o vetor desbalanceamento, para o elemento de casca, é dado por:

$$
\widehat{g}_{j}=\frac{\partial \widehat{\prod}}{\partial \widehat{Y}_{j}}=\int_{\widehat{V}_{0}} \frac{1}{2} \frac{\partial}{\partial \widehat{Y}_{j}}\left(\widehat{E}_{k l} \widehat{C}_{k \lim } \widehat{E}_{i m}\right) d \widehat{V}_{0}-\widehat{F}_{j},
$$

sendo

$$
\frac{1}{2} \frac{\partial}{\partial \widehat{Y}_{j}}\left(\widehat{E}_{k l} \widehat{C}_{k \lim } \widehat{E}_{i m}\right)=\frac{1}{2} \frac{\partial}{\partial \widehat{E}_{\alpha \beta}}\left(\widehat{E}_{k l} \widehat{C}_{k \lim } \widehat{E}_{i m}\right) \frac{\partial \widehat{E}_{\alpha \beta}}{\partial \widehat{Y}_{j}}=\widehat{C}_{\alpha \beta i m} \widehat{E}_{i m} \frac{\partial \widehat{E}_{\alpha \beta}}{\partial \widehat{Y}_{j}} .
$$

Das Eqs. (6.20) e (6.21), tem-se que a matriz Hessiana é dada por:

$$
\left.\frac{\partial \widehat{g}_{j}}{\partial \widehat{Y}_{k}}\right|_{\left(\hat{Y}_{l}^{0}\right)}=\left.\int_{V_{0}} \frac{\partial}{\partial \widehat{Y}_{k}}\left(\widehat{C}_{\alpha \beta i m} \widehat{E}_{i m} \frac{\partial \widehat{E}_{\alpha \beta}}{\partial \widehat{Y}_{j}}\right) d \widehat{V}_{0}\right|_{\left(\hat{Y}_{l}^{0}\right)},
$$

sendo que a derivada dentro da integral resulta em:

$$
\frac{\partial}{\partial \widehat{Y}_{k}}\left(\widehat{\mathbb{C}}_{\alpha \beta i m} \widehat{E}_{i m} \frac{\partial \widehat{E}_{\alpha \beta}}{\partial \widehat{Y}_{j}}\right)=\left(\frac{\partial \widehat{E}_{i m}}{\partial \widehat{Y}_{k}} \widehat{\mathbb{C}}_{\alpha \beta i m} \frac{\partial \widehat{E}_{\alpha \beta}}{\partial \widehat{Y}_{j}}+\widehat{E}_{i m} \widehat{C}_{\alpha \beta i m} \frac{\partial^{2} \widehat{E}_{\alpha \beta}}{\partial \widehat{Y}_{j} \partial \widehat{Y}_{k}}\right)
$$


A derivada primeira e a derivada segunda da deformação de Green em relação aos parâmetros nodais na Eq. (6.23) são dadas, respectivamente, por:

$$
\frac{\partial \hat{\boldsymbol{E}}}{\partial \widehat{Y}_{j}}=\frac{1}{2} \frac{\partial \widehat{\boldsymbol{C}}}{\partial \widehat{Y}_{j}} \mathrm{e} \frac{\partial^{2} \widehat{\boldsymbol{E}}}{\partial \widehat{Y}_{j} \partial \widehat{Y}_{k}}=\frac{1}{2} \frac{\partial^{2} \widehat{\boldsymbol{C}}}{\partial \widehat{Y}_{j} \partial \widehat{Y}_{k}}
$$

onde a derivada primeira do tensor de Cauchy-Green em relação aos parâmetros nodais e a derivada segunda são dadas, respectivamente, por:

$$
\begin{aligned}
& \frac{\partial \widehat{\boldsymbol{C}}}{\partial \widehat{Y}_{j}}=\left(\widehat{\boldsymbol{A}}^{0}\right)^{-T} \cdot \frac{\partial\left(\widehat{\boldsymbol{A}}^{I}\right)^{T}\left(\widehat{Y}_{i}\right)}{\partial \widehat{Y}_{j}} \cdot \widehat{\boldsymbol{A}}^{I}\left(\widehat{Y}_{i}\right) \cdot\left(\widehat{\boldsymbol{A}}^{0}\right)^{-1}+\left(\widehat{\boldsymbol{A}}^{0}\right)^{-T} \cdot\left(\widehat{\boldsymbol{A}}^{I}\right)^{\boldsymbol{T}}\left(\widehat{Y}_{i}\right) \cdot \frac{\partial \widehat{\boldsymbol{A}}^{l}\left(\widehat{Y}_{i}\right)}{\partial \widehat{Y}_{j}} \cdot\left(\widehat{\boldsymbol{A}}^{0}\right)^{-1}, \\
& \frac{\partial^{2} \widehat{\boldsymbol{C}}}{\partial \widehat{Y}_{j} \partial \widehat{Y}_{k}}=\left(\widehat{\boldsymbol{A}}^{0}\right)^{-T} \cdot \frac{\partial\left(\widehat{\boldsymbol{A}}^{I}\right)^{T}\left(\widehat{Y}_{i}\right)}{\partial \widehat{Y}_{j}} \cdot \frac{\partial \widehat{\boldsymbol{A}}^{I}\left(\widehat{Y}_{i}\right)}{\partial \widehat{Y}_{k}} \cdot\left(\widehat{\boldsymbol{A}}^{0}\right)^{-1}+\left(\widehat{\boldsymbol{A}}^{0}\right)^{-T} \cdot \frac{\partial\left(\widehat{\boldsymbol{A}}^{I}\right)^{T}\left(\widehat{Y}_{i}\right)}{\partial \widehat{Y}_{k}} \cdot \frac{\partial \widehat{\boldsymbol{A}}^{I}\left(\widehat{Y}_{i}\right)}{\partial \widehat{Y}_{j}} \cdot\left(\widehat{\boldsymbol{A}}^{0}\right)^{-1}+ \\
& \left(\widehat{\boldsymbol{A}}^{0}\right)^{-T} \cdot\left(\widehat{\boldsymbol{A}}^{I}\right)^{T}\left(\widehat{Y}_{i}\right) \cdot \frac{\partial^{2} \widehat{\boldsymbol{A}}^{I}\left(\widehat{Y}_{i}\right)}{\partial \hat{Y}_{j} \partial \widehat{Y}_{k}} \cdot\left(\widehat{\boldsymbol{A}}^{0}\right)^{-1}+\left(\widehat{\boldsymbol{A}}^{0}\right)^{-T} \cdot \frac{\partial^{2}\left(\widehat{\boldsymbol{A}}^{I}\right)^{T}\left(\widehat{Y}_{i}\right)}{\partial \hat{Y}_{j} \partial \widehat{Y}_{k}} \cdot \widehat{\boldsymbol{A}}^{I}\left(\widehat{Y}_{i}\right) \cdot\left(\widehat{\boldsymbol{A}}^{0}\right)^{-1},
\end{aligned}
$$

sendo $\widehat{A}^{1}$ dado pelas Eqs. (6.16)-(6.18).

\subsection{CINEMÁTICA DO ELEMENTO FINITO DE CASCA LAMINADA}

As expressões para a análise de cascas homogêneas apresentadas na Seção 6.2 são generalizadas nesta seção para a análise de cascas ortotrópicas/anisotrópicas laminadas.

Para isto, considera-se uma casca laminada formada por $m$ lâminas conforme mostrado na Figura 66 cuja cinemática do elemento finito é mostrada na Figura 67. Na Figura 66, $\widehat{d}^{\text {lam é }}$ a distância da superfície de referência do laminado na configuração inicial até o centro de uma lâmina considerada segundo a direção $\xi_{3}$ e $\widehat{h}_{0}^{\text {lam }}$ é a espessura inicial desta lâmina. Da introdução destas considerações, os mapeamentos dados nas Eqs. (6.14) e (6.15) passam a ser dados, respectivamente, por: 


$$
\begin{gathered}
\hat{\varphi}_{i}^{\text {olam }}=\widehat{\phi}_{l}\left(\xi_{1}, \xi_{2}\right) \widehat{X}_{l i}+\left(\hat{d}^{\text {lam }}+\frac{\hat{h}_{0}^{\text {lam }}}{2} \xi_{3}\right) \widehat{\phi}_{l}\left(\xi_{1}, \xi_{2}\right) \widehat{N}_{i l}^{0} \\
\widehat{\varphi}_{i}^{\text {llam }}=\widehat{\phi}_{l}\left(\xi_{1}, \xi_{2}\right) \widehat{Y}_{l i}+\left[\left(\hat{d}^{\text {lam }}+\frac{\hat{h}_{0}^{\text {lam }}}{2} \xi_{3}\right)+\widehat{\phi}_{l}\left(\xi_{1}, \xi_{2}\right) \widehat{a}_{l}\left(\hat{d}^{\text {lam }}+\frac{\hat{h}_{0}^{\text {lam }}}{2} \xi_{3}\right)^{2}\right] \widehat{\phi}_{k}\left(\xi_{1}, \xi_{2}\right) \widehat{G}_{i k}
\end{gathered}
$$

ou seja, o termo $\frac{\widehat{h}_{0}}{2} \xi_{3}$ é substituído por $\widehat{d}^{\text {lam }}+\frac{\widehat{h}_{0}^{\text {lam }}}{2} \xi_{3}$, onde lam indica a lâmina considerada.

A taxa de variação da espessura de cada lâmina, âa lam $_{l}$ pode ser recuperada da representação média de $\widehat{a}_{l}$ pela expressão $\hat{a}_{l}^{\text {lam }}=\left(\hat{h}_{0}^{\text {lam }} / 2\right) \widehat{a}_{l}$.
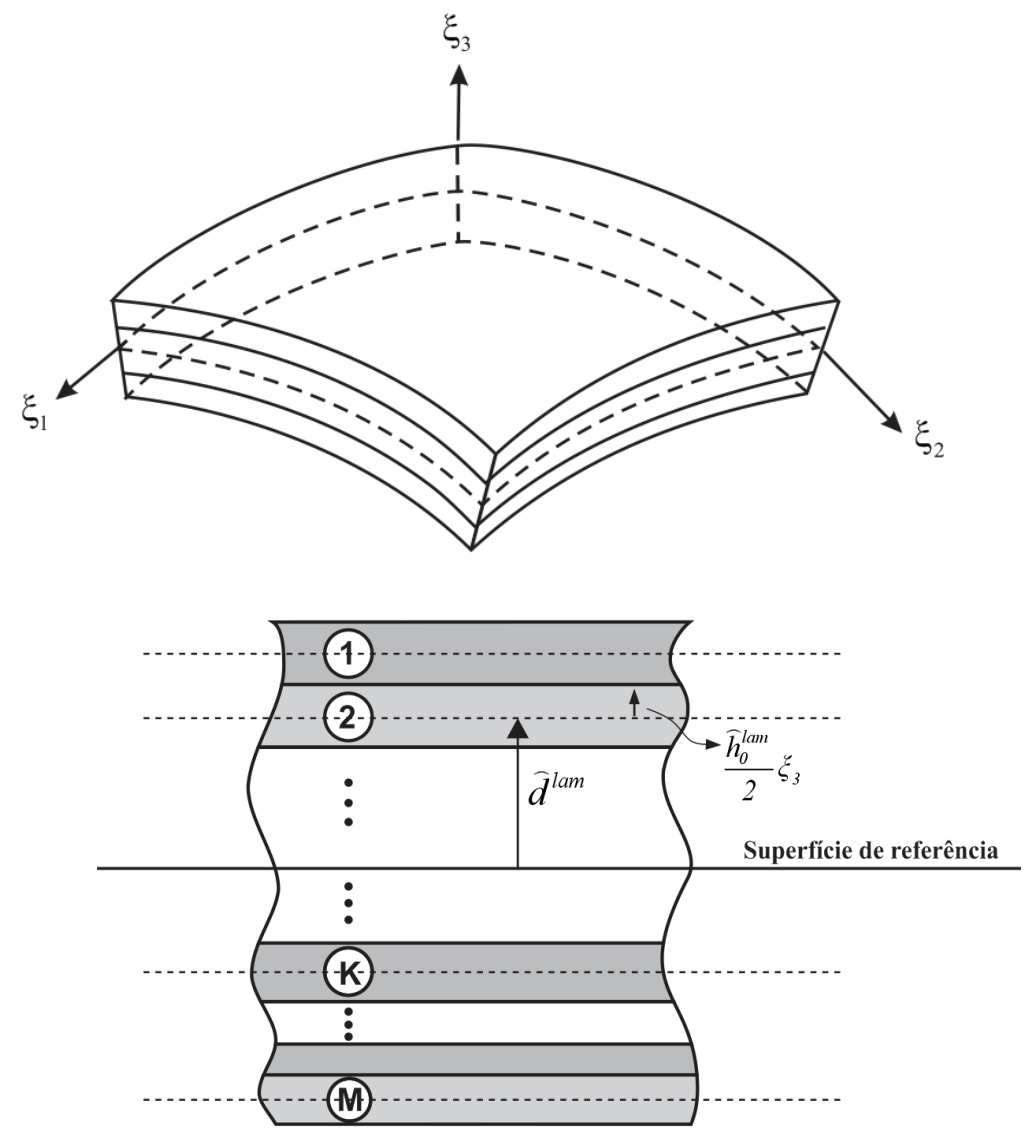

Figura 66 - Casca laminada.

As derivadas de $\hat{\varphi}_{i}^{\text {llam }}$ em relação às variáveis adimensionais são dadas por: 


$$
\begin{aligned}
& \widehat{A}_{i l}^{\text {llam }}=\widehat{\phi}_{l, 1} \widehat{Y}_{l i}+\widehat{\phi}_{l, 1} \widehat{a}_{l}\left(\hat{d}^{\text {lam }}+\frac{\widehat{h}_{0}^{\text {lam }}}{2} \xi_{3}\right)^{2} \widehat{\phi}_{k} \widehat{G}_{i k}+\left[\left(\hat{d}^{\text {lam }}+\frac{\widehat{h}_{0}^{\text {lam }}}{2} \xi_{3}\right)+\widehat{\phi}_{l} \widehat{a}_{l}\left(\hat{d}^{\text {lam }}+\frac{\widehat{h}_{0}^{\text {lam }}}{2} \xi_{3}\right)^{2}\right] \widehat{\phi}_{k, 2} \widehat{G}_{i k} \\
& \widehat{A}_{i 2}^{\text {llam }}=\widehat{\phi}_{l, 2} \widehat{Y}_{l i}+\widehat{\phi}_{l, 2} \widehat{a}_{l}\left(\hat{d}^{\text {lam }}+\frac{\hat{h}_{0}^{\text {lam }}}{2} \xi_{3}\right)^{2} \widehat{\phi}_{k} \widehat{G}_{i k}+\left[\left(\hat{d}^{\text {lam }}+\frac{\hat{h}_{0}^{\text {lam }}}{2} \xi_{3}\right)+\widehat{\phi}_{l} \widehat{a}_{l}\left(\hat{d}^{\text {lam }}+\frac{\widehat{h}_{0}^{\text {lam }}}{2} \xi_{3}\right)^{2}\right] \widehat{\phi}_{k, 2} \widehat{G}_{i k} \\
& \widehat{A}_{i 3}^{\text {llam }}=\frac{\widehat{h}_{0}}{2}\left[1+\widehat{\phi}_{l} \widehat{a}_{l}\left(2 \widehat{d}^{\text {lam }}+\widehat{h}_{0}^{\text {lam }} \xi_{3}\right)\right] \widehat{\phi}_{k} \widehat{G}_{i k}
\end{aligned}
$$

Exemplos de validação desta cinemática para a análise de cascas laminadas foram apresentados por Coda, Paccola e Sampaio (2013).

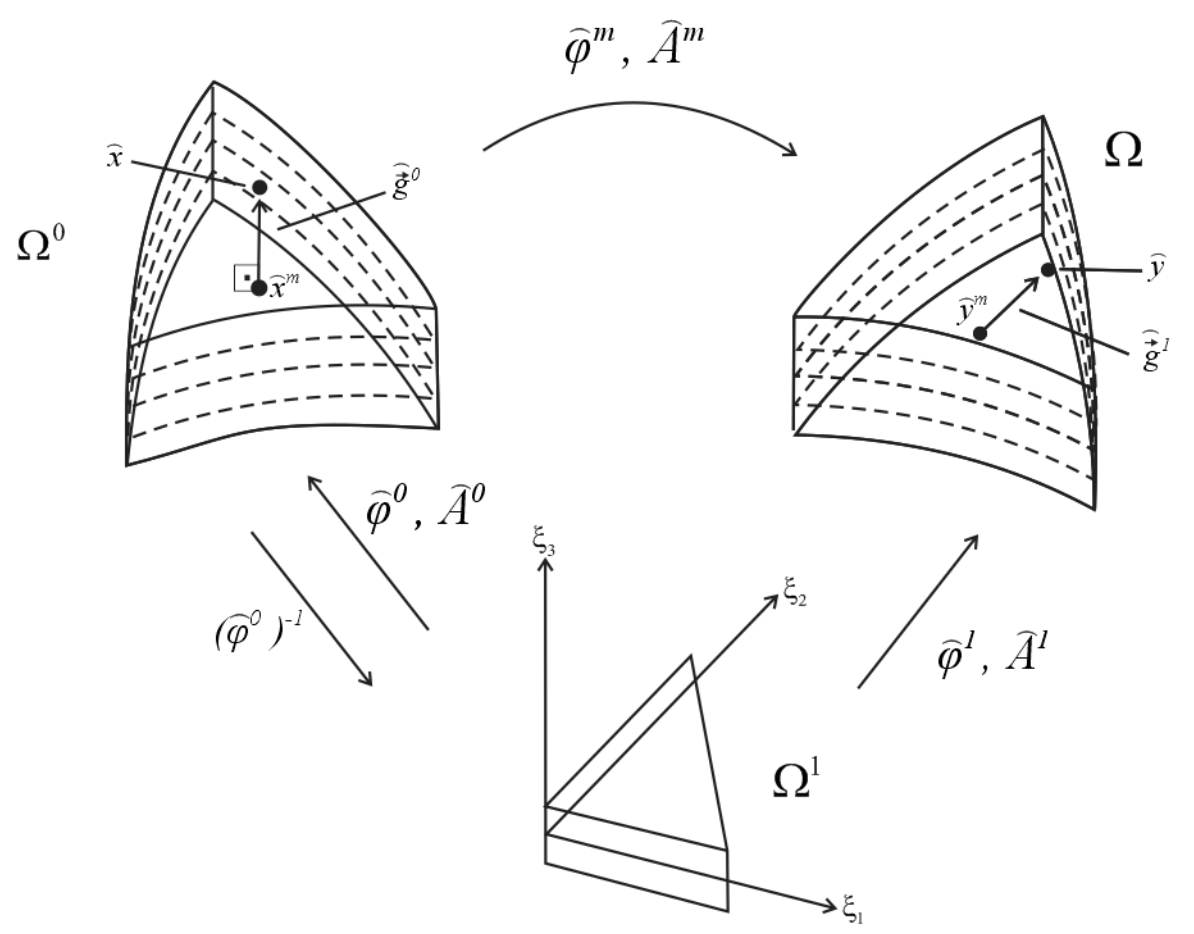

Figura 67 - Cinemática para cascas laminadas.

\subsection{FIBRAS PARA CASCAS LAMINADAS}

O elemento finito de fibra curvo de ordem qualquer utilizado para a análise de laminados reforçados com fibras é o mesmo desenvolvido na Seção 4.4, porém com três parâmetros por nó, as posições $\tilde{X}_{1}^{p}, \tilde{X}_{2}^{p}$ e $\tilde{X}_{3}^{p}$. Desta forma, os módulos dos vetores 
tangentes associados a um ponto da fibra curva na configuração inicial e atual e a deformação de Green-Lagrange dados, respectivamente, pelas Eqs. (4.24), (4.25) e (4.27) passam a ser expressos, respectivamente, por:

$$
\begin{gathered}
\left|\vec{T}^{B_{0}}\right|^{2}=\left(\frac{d \tilde{\phi}_{p}(\eta)}{d \eta} \tilde{X}_{1}^{p}\right)^{2}+\left(\frac{d \tilde{\phi}_{p}(\eta)}{d \eta} \tilde{X}_{2}^{p}\right)^{2}+\left(\frac{d \tilde{\phi}_{p}(\eta)}{d \eta} \tilde{X}_{3}^{p}\right)^{2}, \\
\left|\vec{T}^{B}\right|^{2}=\left(\frac{d \tilde{\phi}_{p}(\eta)}{d \eta} \tilde{Y}_{1}^{p}\right)^{2}+\left(\frac{d \tilde{\phi}_{p}(\eta)}{d \eta} \tilde{Y}_{2}^{p}\right)^{2}+\left(\frac{d \tilde{\phi}_{p}(\eta)}{d \eta} \tilde{Y}_{3}^{p}\right)^{2}, \\
\tilde{E}=\frac{1}{2} \frac{\left[\left(\frac{d \tilde{\phi}_{p}(\eta)}{d \eta} \tilde{Y}_{1}^{p}\right)^{2}+\left(\frac{d \tilde{\phi}_{p}(\eta)}{d \eta} \tilde{Y}_{2}^{p}\right)^{2}+\left(\frac{d \tilde{\phi}_{p}(\eta)}{d \eta} \tilde{Y}_{3}^{p}\right)^{2}\right]-\left[\left(\frac{d \tilde{\phi}_{p}(\eta)}{d \eta} \tilde{X}_{1}^{p}\right)^{2}+\left(\frac{d \tilde{\phi}_{p}(\eta)}{d \eta} \tilde{X}_{2}^{p}\right)^{2}+\left(\frac{d \tilde{\phi}_{p}(\eta)}{d \eta} \tilde{X}_{3}^{p}\right)^{2}\right] .}{\left(\frac{d \tilde{\phi}_{p}(\eta)}{d \eta} \tilde{X}_{1}^{p}\right)^{2}+\left(\frac{d \phi_{p}(\eta)}{d \eta} \tilde{X}_{2}^{p}\right)^{2}+\left(\frac{d \tilde{\phi}_{p}(\eta)}{d \eta} \tilde{X}_{3}^{p}\right)^{2}}
\end{gathered}
$$

Os demais equacionamentos necessários para a obtenção do vetor de forças internas e da matriz Hessiana são análogos aos desenvolvidos naquela seção, com exceção para o acoplamento fibra-casca laminada apresentado a seguir.

\subsection{ACOPLAMENTO FIBRA-CASCA LAMINADA}

Nesta seção descreve-se a estratégia adotada para inserir a contribuição das fibras, dispostas nas diversas lâminas de uma dada casca laminada, na energia total do sistema. Destaca-se que as fibras podem estar em qualquer posição ao longo da espessura de cada lâmina conforme representado na Figura 68. Esta estratégia é uma extensão da estratégia apresentada na Seção 4.6.

As posições $\tilde{X}_{i}^{p}$ dos nós das fibras curvas na configuração inicial e as posições $\tilde{Y}_{i}^{p}$ dos nós das fibras curvas na configuração atual são escritas em funções dos parâmetros nodais do elemento de casca laminada, respectivamente, como:

$$
\tilde{X}_{i}^{p}=\widehat{\phi}_{l}\left(\xi_{1}^{p}, \xi_{2}^{p}\right) \widehat{X}_{l i}+\left(\widehat{d}^{l a m}+\frac{\widehat{h}_{0}^{\text {lam }}}{2} \xi_{3}^{p}\right) \widehat{\phi}_{l}\left(\xi_{1}^{p}, \xi_{2}^{p}\right) \widehat{N}_{i l}^{0}
$$




$$
\tilde{Y}_{i}^{p}=\widehat{\phi}_{l}\left(\xi_{l}^{p}, \xi_{2}^{p}\right) \widehat{Y}_{l i}+\left[\left(\widehat{d}^{l a m}+\frac{\hat{h}_{0}^{l a m}}{2} \xi_{3}^{p}\right)+\widehat{\phi}_{l}\left(\xi_{1}^{p}, \xi_{2}^{p}\right) \widehat{a}_{l}\left(\widehat{d}^{\text {lam }}+\frac{\widehat{h}_{0}^{l a m}}{2} \xi_{3}^{p}\right)^{2}\right] \widehat{\phi}_{k}\left(\xi_{l}^{p}, \xi_{2}^{p}\right) \widehat{G}_{i k}
$$
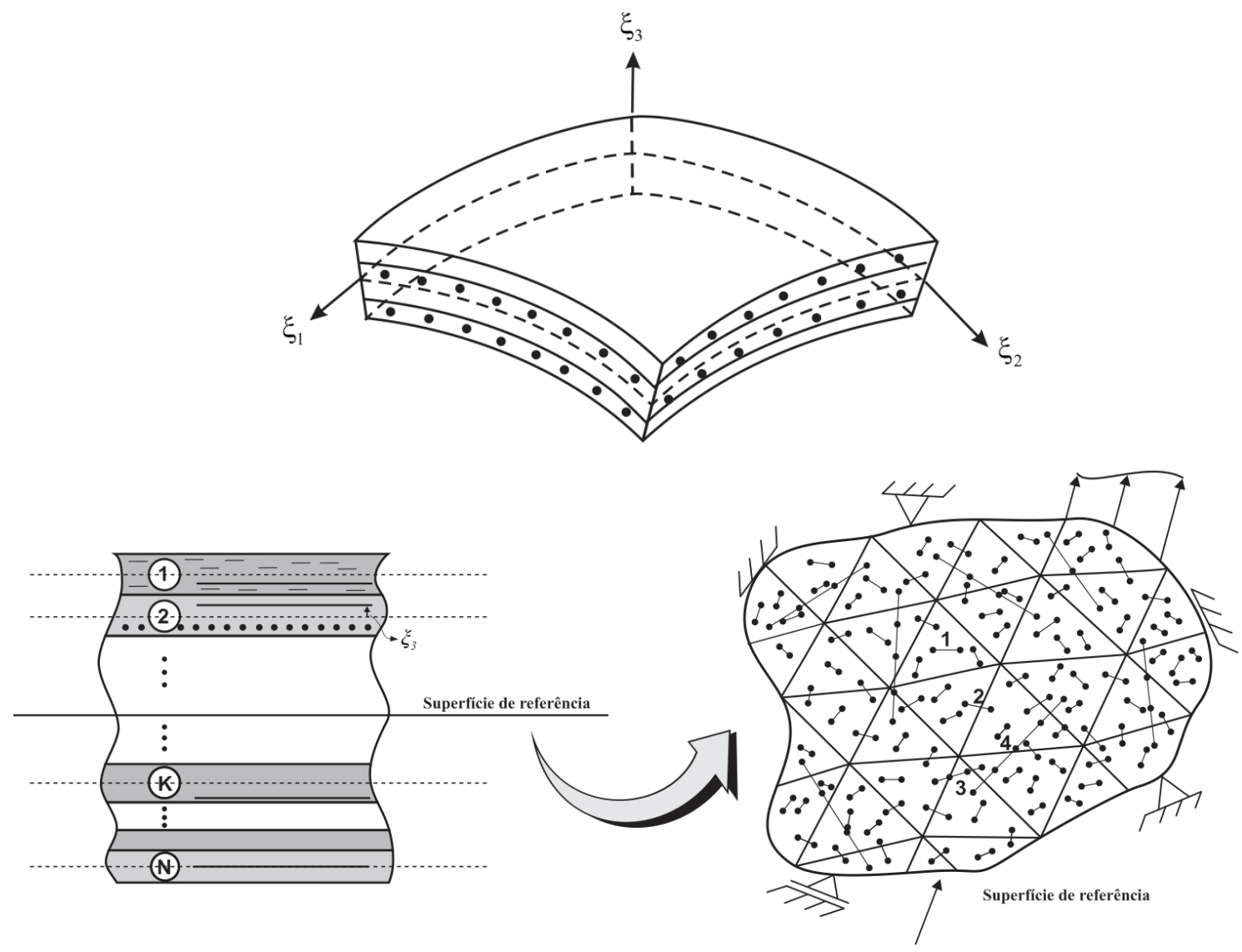

Figura 68 - Discretização fibra-matriz da superfície de referência do laminado.

Da mesma forma que para o caso bidimensional, a energia de deformação armazenada em um laminado reforçado com fibras é dada pela soma da energia de deformação armazenada na matriz discretizada com elementos finitos de casca laminada e da energia de deformação armazenada nas fibras, lembrando que neste caso, o elemento finito de casca laminada possui dez nós e sete parâmetros por nó e as fibras três parâmetros por nó.

Além das coordenadas adimensionais $\xi_{1}^{p} e \xi_{2}^{p}$ relacionadas à superfície de referência da casca, as fibras possuem um terceiro parâmetro, $-1 \leq \xi_{3}^{p} \leq+1$, associado às posições que ocupam ao longo da espessura da camada onde estão contidas.

Da mesma forma como descrito na Seção 4.5.2 e 4.5.3 para o caso de domínios bidimensionais, os diferentes valores que o termo $\frac{\partial \tilde{Y}_{n}^{\eta}}{\partial \tilde{Y}_{\gamma}^{\xi}}$ na Eq. (4.53) pode assumir ao realizar a segunda derivada da energia de deformação para a obtenção da matriz Hessiana, considerando 
elemento de fibra curvo e elemento de casca laminada, dependem da discretização adotada, ou seja, das configurações apresentadas pelas fibras em relação à superfície de referência do laminado (Figura 68).

Assim, para uma fibra curva com ordem de aproximação GP $^{f}$ com três parâmetros por nó e um elemento de casca com $\widehat{N}$ nós e sete parâmetros por nó, a matriz dada pela Eq. (4.56) passa a ser dada por:

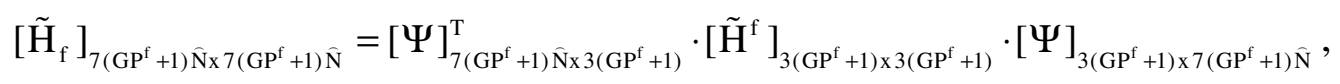

sendo $\left[\tilde{\mathrm{H}}^{\mathrm{f}}\right]_{3\left(\mathrm{GP}^{\mathrm{f}}+1\right) \times 3\left(\mathrm{GP}^{\mathrm{f}}+1\right)}$ a matriz Hessiana local da fibra curva de ordem qualquer obtida da Eq. (4.39) com parâmetros nodais variando de 1 a 3 para o caso de fibra curva na casca. $\mathrm{O}$ subscrito $\mathrm{f}$ em $\left[\tilde{\mathrm{H}}_{\mathrm{f}}\right]$ e o sobrescrito $\mathrm{f}$ em $\left[\tilde{\mathrm{H}}^{\mathrm{f}}\right]$ na Eq. (6.37) fazem distinção entre a matriz Hessiana expandida da fibra curva a ser contribuída na matriz Hessiana global da casca e a matriz Hessiana local da fibra curva, respectivamente.

A matriz esparsa $[\Psi]_{3\left(\mathrm{GP}^{\mathrm{f}}+1\right) \times 7\left(\mathrm{GP}^{\mathrm{f}}+1\right) \hat{\mathrm{N}}}$ na Eq. $(6.37)$ tem a forma:

$$
[\Psi]=\left[\begin{array}{ccccccc}
{[\Theta]_{3 \times 7 \widehat{N}}^{1}} & 0 & 0 & 0 & 0 & 0 & 0 \\
0 & {[\Theta]_{3 \times 7 \widehat{N}}^{2}} & 0 & 0 & 0 & 0 & 0 \\
0 & 0 & {[\Theta]_{3 \times 7 \widehat{N}}^{3}} & 0 & 0 & 0 & 0 \\
0 & 0 & 0 & \ddots & 0 & 0 & 0 \\
0 & 0 & 0 & 0 & {[\Theta]_{3 \times 7 \hat{N}}^{\mathrm{GP}^{\mathrm{f}}-1}} & 0 & 0 \\
0 & 0 & 0 & 0 & 0 & {[\Theta]_{3 \times 7 \widehat{N}}^{\mathrm{GP}^{\mathrm{f}}}} & 0 \\
0 & 0 & 0 & 0 & 0 & 0 & {[\Theta]_{3 \times 7 \widehat{N}}^{\mathrm{GP}^{\mathrm{f}}+1}}
\end{array}\right]_{3\left(\mathrm{GP}^{\mathrm{f}}+1\right) \times 7\left(\mathrm{GP}^{\mathrm{f}}+1\right) \hat{\mathrm{N}}}
$$

Por sua vez, cada termo da matriz na Eq. (6.38) tem a forma:

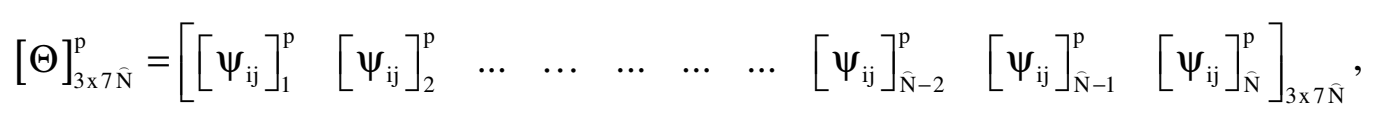

onde $p$ é o nó de fibra considerado.

Para uma fibra cúbica, $\left[\tilde{\mathrm{H}}^{\mathrm{f}}\right]_{12 \times 12}$, e um elemento de casca com 10 nós, a matriz dada na 
Eq. (6.37) é dada por:

$$
\left[\tilde{\mathrm{H}}_{\mathrm{f}}\right]_{280 \times 280}=[\Psi]_{280 \times 12}^{\mathrm{T}} \cdot\left[\mathrm{H}^{\mathrm{f}}\right]_{12 \times 12} \cdot[\Psi]_{12 \times 280} .
$$

Para uma fibra cúbica a matriz esparsa $[\Psi]$ na Eq. (6.40) é dada por:

$$
[\Psi]=\left[\begin{array}{cccc}
{[\Theta]_{3 \times 70}^{1}} & 0 & 0 & 0 \\
0 & {[\Theta]_{3 \times 70}^{2}} & 0 & 0 \\
0 & 0 & {[\Theta]_{3 \times 70}^{3}} & 0 \\
0 & 0 & 0 & {[\Theta]_{3 \times 70}^{4}}
\end{array}\right]_{12 \times 280}
$$

onde os termos do tipo $[\Theta]_{3 \times 70}^{p}$ na Eq. (6.41) são dados pela contribuição do nó $p$ de fibra considerado em relação a todos os nós do elemento finito de casca que contém o nó $p$ e são dadas por:

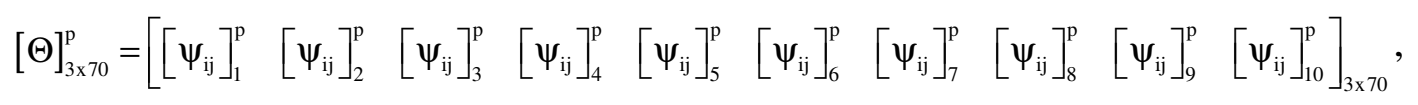

sendo $i$ os graus de liberdade dos nós do elemento de fibra e $j$ os graus de liberdade dos nós do elemento de casca. Desta forma, cada termo $\left[\psi_{\mathrm{ij}}\right]_{\beta}^{\mathrm{p}}$ na Eq. (6.42) ou Eq. (6.39) tem a forma:

$$
\left[\psi_{\mathrm{ij}}\right]_{\beta}^{\mathrm{p}}=\left[\begin{array}{ccccccc}
\psi_{11} & 0 & 0 & \psi_{14} & \psi_{15} & 0 & 0 \\
0 & \psi_{22} & 0 & \psi_{24} & 0 & \psi_{26} & 0 \\
0 & 0 & \psi_{33} & \psi_{34} & 0 & 0 & \psi_{37}
\end{array}\right]_{3 \times 7},
$$

sendo $\beta$ os nós do elemento finito de casca que contém o nó $p$ da fibra considerado.

Os termos $\psi_{\mathrm{ij}}$ na Eq. (6.43) são obtidos a partir da Eq. (6.36). Desta forma, lembrando que $\hat{Y}_{4}^{k}=\hat{a}^{k}, \hat{Y}_{5}^{k}=\widehat{G}_{1}^{k}, \hat{Y}_{6}^{k}=\widehat{G}_{2}^{k}$ e $\hat{Y}_{7}^{k}=\widehat{G}_{3}^{k}$, as derivadas da Eq. (6.36) em relação aos parâmetros nodais da casca são dadas por: 


$$
\begin{gathered}
\psi_{i(i)}=\frac{\partial \tilde{Y}_{i}^{p}}{\partial \hat{Y}_{(i)}^{\beta}}=\widehat{\phi}_{\beta}\left(\xi_{1}^{p}, \xi_{2}^{p}\right), \\
\psi_{i 4}=\frac{\partial \tilde{Y}_{i}^{p}}{\partial \hat{Y}_{4}^{\beta}}=\left[\widehat{\phi}_{\beta}\left(\xi_{1}^{p}, \xi_{2}^{p}\right)\left(\hat{d}^{\text {lam }}+\frac{\hat{h}_{0}^{\text {lam }}}{2} \xi_{3}^{p}\right)^{2}\right] \widehat{\phi}_{k}\left(\xi_{1}^{p}, \xi_{2}^{p}\right) \widehat{Y}_{i+4}^{k}, \\
\psi_{i(i+4)}=\frac{\partial \tilde{Y}_{i}^{p}}{\partial \widehat{Y}_{(i+4)}^{\beta}}=\left[\left(\hat{d}^{\text {lam }}+\frac{\hat{h}_{0}^{\text {lam }}}{2} \xi_{3}^{p}\right)+\widehat{\phi}_{\ell}\left(\xi_{1}^{p}, \xi_{2}^{p}\right) \widehat{Y}_{4}^{\ell}\left(\hat{d}^{\text {lam }}+\frac{\widehat{h}_{0}^{\text {lam }}}{2} \xi_{3}^{p}\right)^{2}\right] \widehat{\phi}_{\beta}\left(\xi_{1}^{p}, \xi_{2}^{p}\right),
\end{gathered}
$$

com $i=1,2,3$.

Os termos $\psi_{\mathrm{ij}}$ na Eq. (6.43) podem ainda ser obtidos a partir da Eq. (6.36), desprezando-se a taxa de variação da espessura, ou seja,

$$
\begin{gathered}
\psi_{i(i)}=\frac{\partial \tilde{Y}_{i}^{p}}{\partial \widehat{Y}_{(i)}^{\beta}}=\widehat{\phi}_{\beta}\left(\xi_{1}^{p}, \xi_{2}^{p}\right), \\
\psi_{i 4}=\frac{\partial \tilde{Y}_{i}^{p}}{\partial \widehat{Y}_{4}^{\beta}}=0, \\
\psi_{i(i+4)}=\frac{\partial \tilde{Y}_{i}^{p}}{\partial \hat{Y}_{(i+4)}^{\beta}}=\left[\left(\hat{d}^{\text {lam }}+\frac{\widehat{h}_{0}^{\text {lam }}}{2} \xi_{3}^{p}\right)\right] \widehat{\phi}_{\beta}\left(\xi_{1}^{p}, \xi_{2}^{p}\right),
\end{gathered}
$$

com $i=1,2,3$ e assim a Eq. (6.43) passa a ser dada por:

$$
\left[\psi_{\mathrm{ij}}\right]_{\beta}^{\mathrm{p}}=\left[\begin{array}{ccccccc}
\psi_{11} & 0 & 0 & 0 & \psi_{15} & 0 & 0 \\
0 & \psi_{22} & 0 & 0 & 0 & \psi_{26} & 0 \\
0 & 0 & \psi_{33} & 0 & 0 & 0 & \psi_{37}
\end{array}\right]_{3 \times 7}
$$

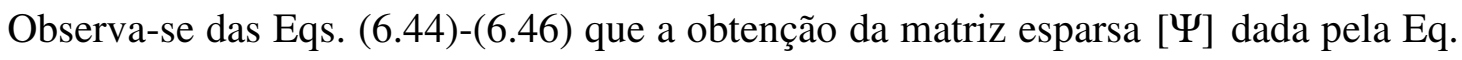
(6.41) ou Eq. (6.38) depende dos valores de $\widehat{\phi}_{\beta}\left(\xi_{1}^{p}, \xi_{2}^{p}, \xi_{3}^{p}, d_{\text {lam }}\right)$ e do elemento de casca que contém o nó de fibra $P$ considerado. A matriz Hessiana expandida $\left[\tilde{\mathrm{H}}_{\mathrm{f}}\right]$ dada pela Eq. (6.40) ou Eq. (6.37) é distribuída na matriz Hessiana total do sistema seguindo a conectividade da casca associada ao nó de fibra considerado.

Neste trabalho, o procedimento para a determinação das posições das fibras em cascas é feito de forma indireta, ou seja, determinam-se as coordenadas adimensionais $\xi_{i}^{p}$ dos nós 
das fibras e o elemento finito bidimensional onde estes nós estão contidos em uma malha auxiliar plana que serve de base para o mapeamento da casca. Desta forma, as fibras são transferidas automaticamente para a sua posição na casca seguindo o mesmo mapeamento utilizado para a sua geração na malha auxiliar.

\subsection{EXEMPLOS NUMÉRICOS}

Para demonstrar a aplicabilidade da estratégia introduzida neste trabalho são apresentados três exemplos numéricos. O primeiro consiste em uma viga reforçada por quatro enrijecedores submetida a grandes deslocamentos, o segundo exemplo consiste em uma placa simplesmente apoiada reforçada por fibras em uma e em duas direções e o terceiro uma casca laminada cilíndrica reforçada com fibras submetida a pequenos e grandes deslocamentos.

\subsubsection{EXEMPLO 1: VIGA ENGASTADA REFORÇADA COM FIBRAS SUBMETIDA A GRANDES DESLOCAMENTOS}

Neste exemplo uma viga engastada solicitada por um carregamento uniformemente distribuído $q$ é analisada. A viga está reforçada por quatro enrijecedores dispostos conforme mostrado na Figura 69. As propriedades geométricas adotadas para a viga são dadas por: $L=300 \mathrm{~cm}, b=20 \mathrm{~cm}, h=60 \mathrm{~cm}, d=3 \mathrm{~cm}$ e $q=5,0 \mathrm{MN} / \mathrm{m}$. O módulo de elasticidade e o coeficiente de Poisson são dados, respectivamente, por $E_{m}=21 G P a$ e $v_{m}=0,0$. Para as fibras, o módulo de elasticidade e a área da seção transversal são dados, respectivamente, por $E_{f}=210 G P a$ e $A_{f}=2 \mathrm{~cm}^{2}$.

Os resultados obtidos com a formulação proposta neste trabalho são comparados com resultados obtidos por três diferentes formulações: a) a primeira denominada como barra geral 3D utiliza cinemática de Timoshenko-Reissner e foi apresentada por Coda (2009), Coda e Paccola (2010) e Coda e Paccola (2011); b) a segunda apresentada por Sampaio, Paccola e Coda (2013) consiste em uma formulação para a análise de domínios bidimensionais reforçados com fibras apresentada no Capítulo 4; e c) a terceira apresentada por Coda, 
Paccola e Sampaio (2013) destina-se a análise de cascas laminadas.

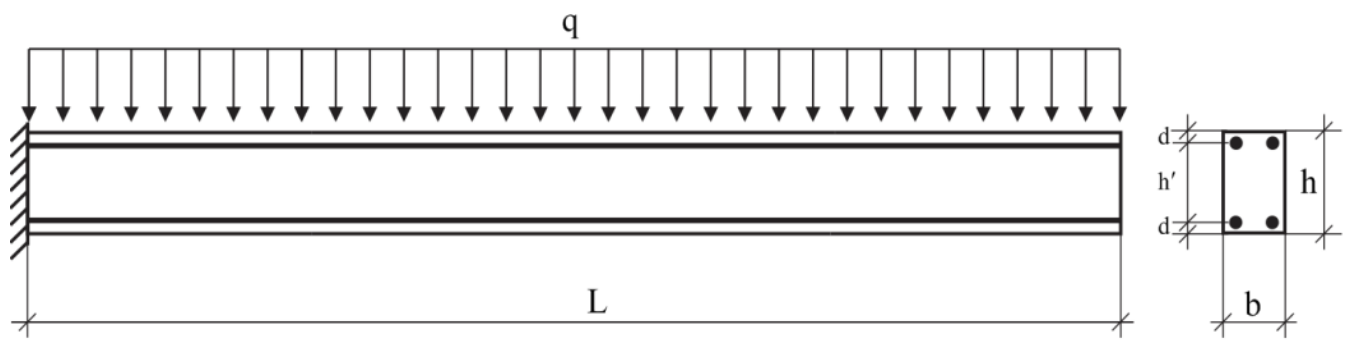

Figura 69 - Viga engastada reforçada com fibras.

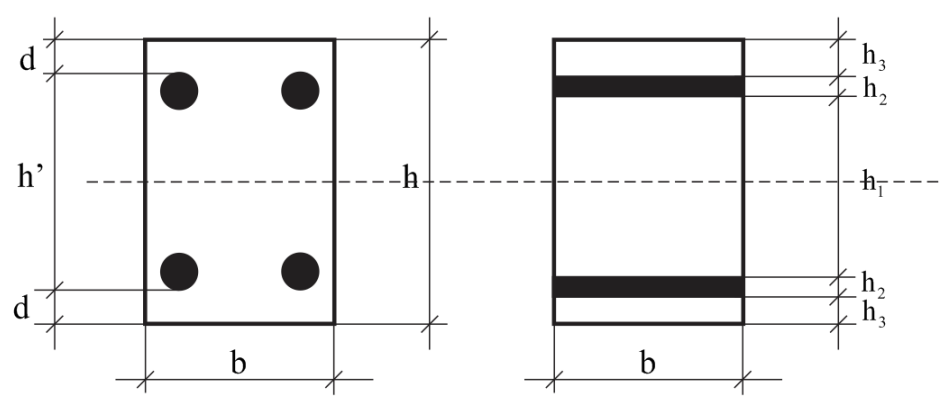

( a )

(b)

Figura 70 - Geometria da seção transversal: (a) original, (b) laminada equivalente.

Para as análises realizadas com a formulação proposta neste trabalho, a formulação de cascas laminadas e a formulação para a análise de domínios bidimensionais reforçados com fibras a matriz foi discretizada em 60 elementos finitos triangulares com aproximação cúbica para as variáveis totalizando 364 nós. Para a análise com a formulação de barra geral 3D a viga foi discretizada em 10 elementos finitos do quinto grau.

Na Figura 71 é possível observar a configuração final da viga após a deformação para as diferentes formulações consideradas e a Figura 72 compara os deslocamentos transversais obtidos.

Os deslocamentos máximos na extremidade da viga reforçada com fibras bem como as diferenças relativas obtidas entre as formulações em relação à solução de referência (barra geral 3D) são mostradas na Tabela 7. Observa-se que as soluções obtidas pela formulação proposta neste trabalho e pela formulação de cascas laminadas estão bem próximas à solução de referência. A grande diferença relativa apresentada pela formulação para análise de domínios bidimensionais reforçados com fibras ocorre, pois a solução de referência (barra geral 3D) apresenta mais restrições cinemáticas (Timoshenko-Reissner) e a altura da barra analisada está acima do limite de representatividade da cinemática de Reissner. A partir dos resultados obtidos é possível observar que a formulação proposta neste trabalho apresenta 
resultados satisfatórios para análises em grandes deslocamentos.
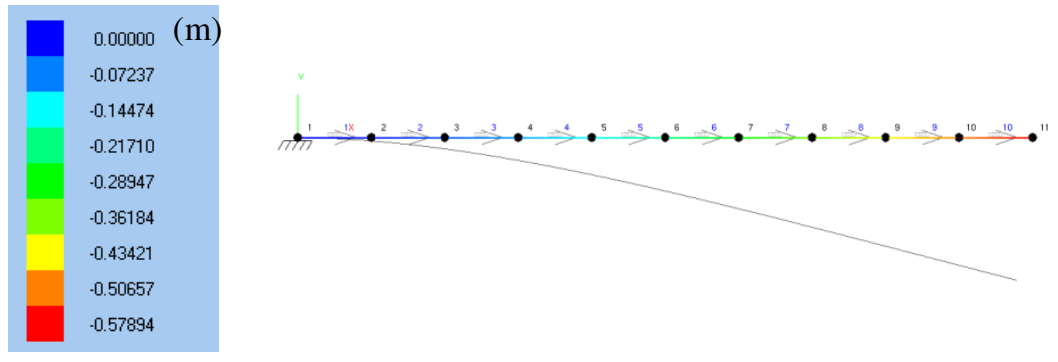

(a)
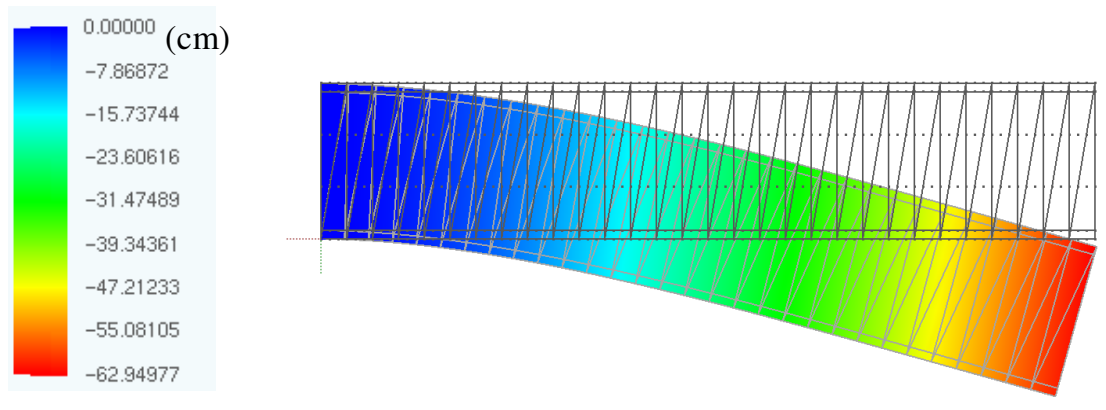

(b)
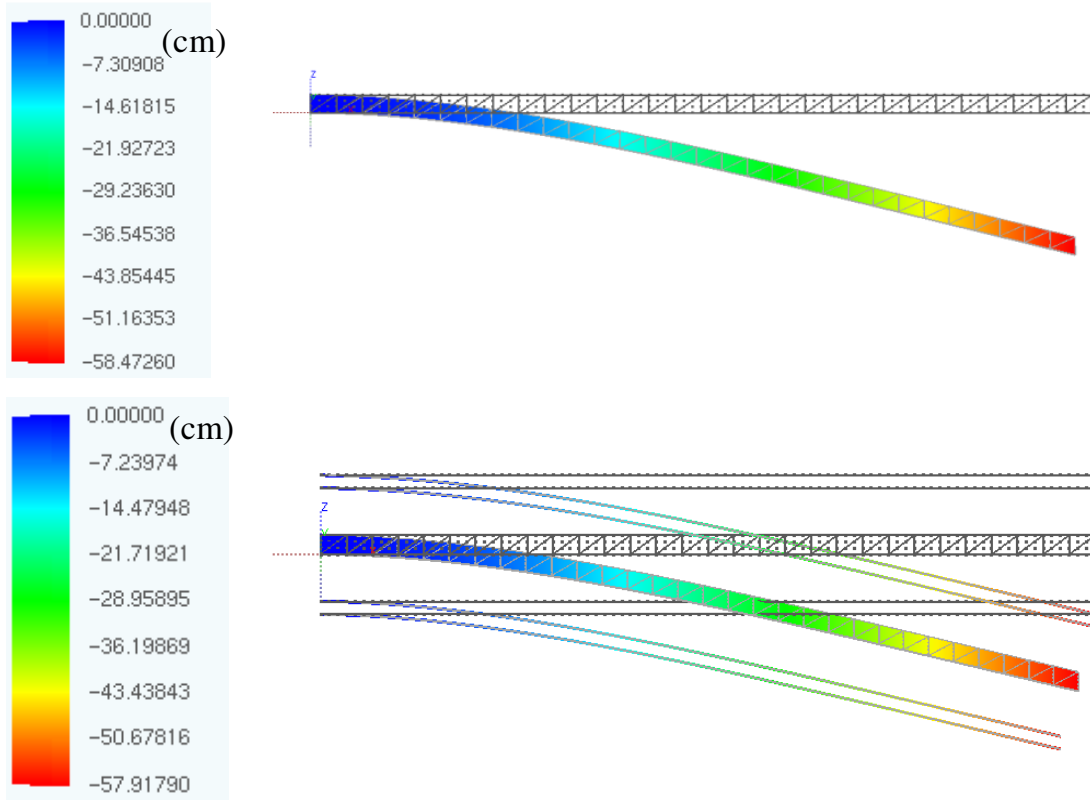

(c)

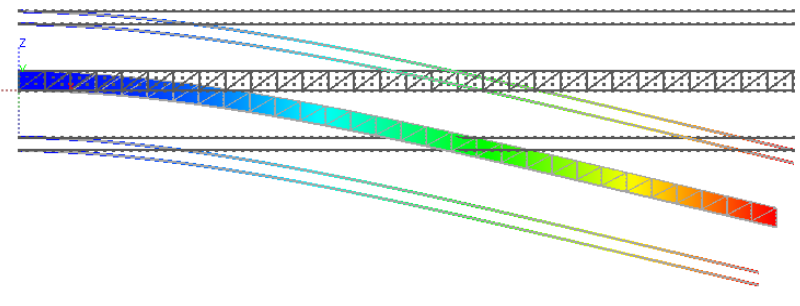

(d)

Figura 71 - Deslocamentos transversais da viga: (a) barra geral 3D; (b) domínio 2D; (c) placa laminada equivalente; (d) placa com fibras. 


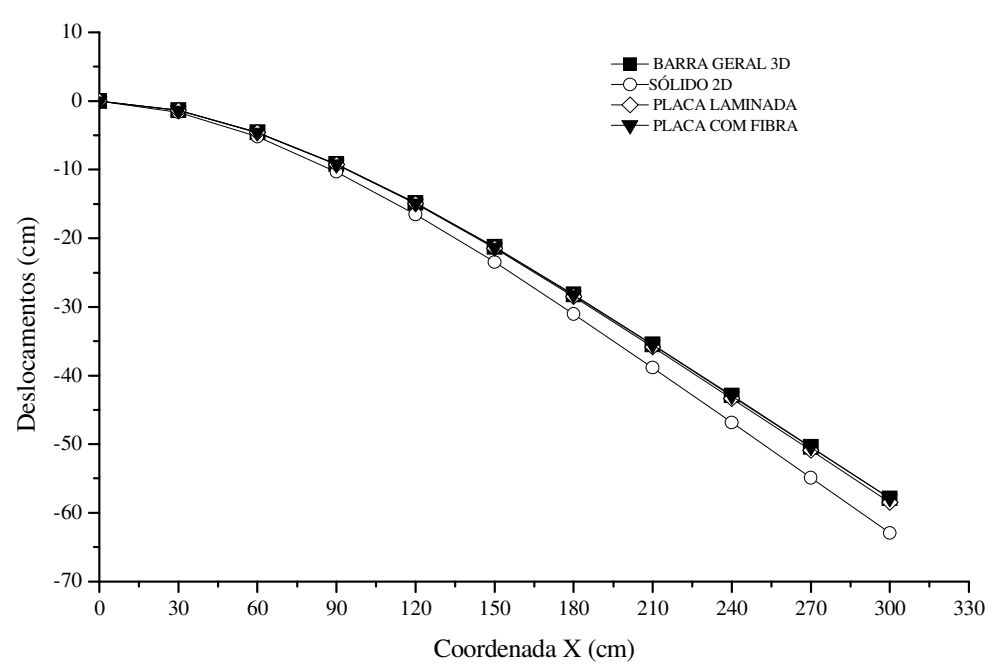

Figura 72 - Deslocamento transversal da viga em $(\mathrm{cm})$.

Tabela 7 - Valores numéricos para o deslocamento transversal máximo em $(\mathrm{cm})$.

\begin{tabular}{|c|c|c|c|}
\hline \multirow{2}{*}{$\begin{array}{l}\text { BARRA GERAL 3D } \\
\text { (solução de referência) }\end{array}$} & \multirow[b]{2}{*}{ DOMÍNIO 2D } & \multicolumn{2}{|c|}{ VIGA } \\
\hline & & $\begin{array}{l}\text { LAMINANADA } \\
\text { EQUIVALENTE }\end{array}$ & COM FIBRAS \\
\hline$-57,894$ & $-62,949$ & $-58,472$ & $-57,917$ \\
\hline Diferença relativa & $\sim 8,03 \%$ & $\sim 0,98 \%$ & $\sim 0,039 \%$ \\
\hline
\end{tabular}

\subsubsection{EXEMPLO 2: PLACA SIMPLESMENTE APOIADA REFORÇADA COM FIBRAS}

Neste exemplo uma placa quadrada simplesmente apoiada com lado $L=400 \mathrm{~cm}$ e espessura $h=16 \mathrm{~cm}$ submetida a um carregamento uniformemente distribuído, $q=-1,0 \mathrm{kN} / \mathrm{m}^{2}$, é analisada (Figura 73).

Dois casos são considerados: no primeiro, a placa é reforçada com fibras dispostas segundo a direção $X_{1}$, Figura 73a; no segundo, a placa é reforçada com fibras dispostas segundo duas direções, $X_{1}$ e $X_{2}$, Figura 73b. O módulo de elasticidade e o coeficiente de Poisson adotados para a matriz são dados, respectivamente, por $E_{m}=21 G P a$ e $v_{m}=0,25$. O módulo de elasticidade e a área da seção transversal de cada fibra são dados, respectivamente, 
por $E_{f}=210 G P a$ e $A_{f}=2 \mathrm{~cm}^{2}$. O centro de gravidade de cada fibra está localizado a uma distância $d=2 \mathrm{~cm}$, medida a partir da base inferior da placa.

Devido à dupla simetria, somente um quarto do problema foi discretizado. Desta forma, a superfície de referência da matriz foi discretizada em uma malha regular com 200 elementos finitos triangulares, com aproximação cúbica para as variáveis, totalizando 961 nós, Figura 74a. Para a placa reforçada em uma direção, cada uma das 20 fibras longas foi discretizada em 10 elementos finitos cúbicos, Figura 74b. Analogamente, para a placa reforçada em duas direções, cada direção possui 20 fibras longas discretizadas em 10 elementos finitos com aproximação cúbica para as variáveis, Figura 74c. Em ambos os casos o espaçamento entre as fibras é $10 \mathrm{~cm}$.

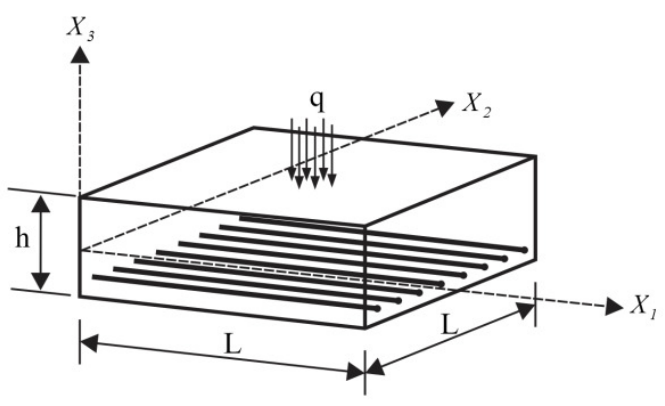

(a)

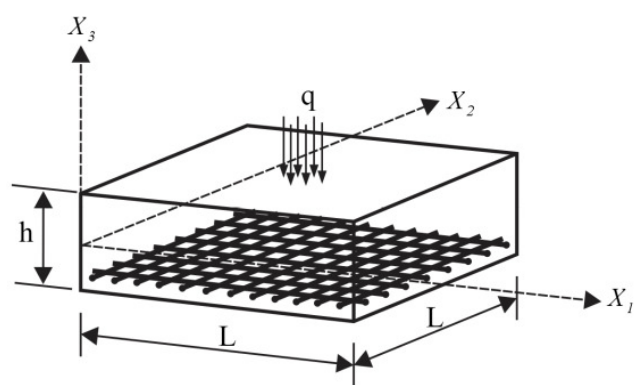

(b)

Figura 73 - Placa quadrada simplesmente apoiada reforçada com fibras: (a) reforçada em uma direção; (b) reforçada em duas direções.

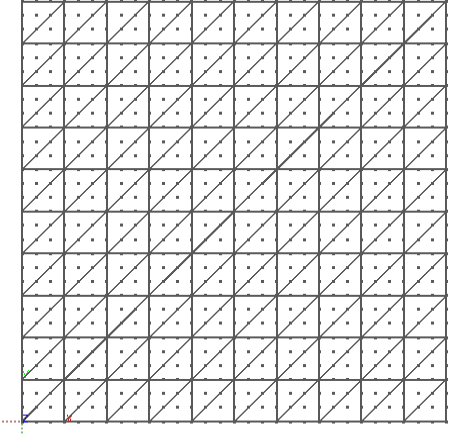

(a)

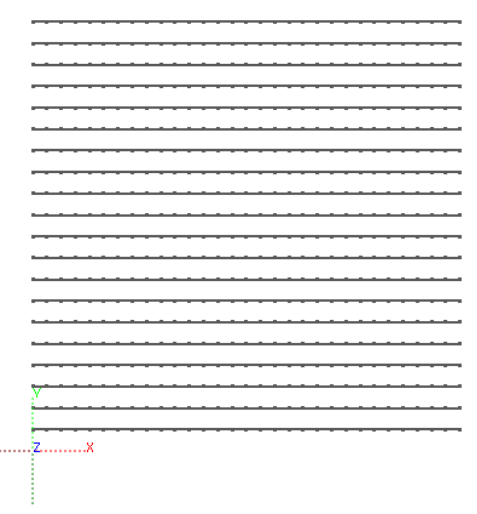

(b)

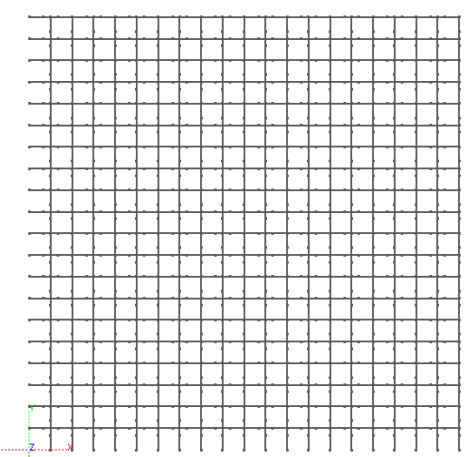

(c)

Figura 74 - Discretização para um quarto do problema: (a) matriz; (b) fibras para placa reforçada em uma direção; (c) fibras para placa reforçada em duas direções.

Os resultados obtidos com a formulação introduzida neste trabalho são comparados com resultados obtidos com uma formulação de referência (CODA; PACCOLA; SAMPAIO, 
2013) em que a seção transversal original reforçada com fibras é simulada como uma seção laminada equivalente. Neste caso, a seção transversal da placa foi discretizada em cinco camadas. Para a placa reforçada em uma direção, adotou-se, para a camada equivalente às fibras, $E_{11}=210 G P a, E_{22}=E_{33}=21 G P a, v_{12}=v_{13}=v_{23}=0,25, G_{12}=84 G P a, G_{13}=G_{23}=8,4 G P a \mathrm{e}$ $h=0,195 \mathrm{~cm}$. Para a placa reforçada em duas direções adotou-se, para a camada equivalente às fibras, $E_{11}=E_{22}=210 G P a, E_{33}=21 G P a, v_{12}=v_{13}=v_{23}=0,25, G_{12}=G_{13}=84 G P a, G_{23}=8,4 G P a$ e $h=0,195 \mathrm{~cm}$. Em ambos os casos adotou-se $E_{11}=E_{22}=E_{33}=21 G P a, v_{12}=v_{13}=v_{23}=0,25 \mathrm{e}$ $G_{l 2}=G_{13}=G_{23}=8,4 G P a$ para as outras camadas. Foram utilizados dez pontos de Gauss para a integração ao longo da espessura do laminado.

A Figura 75 mostra as distribuições dos deslocamentos transversais da placa para os diferentes casos analisados e a Tabela 8 compara seus valores máximos.
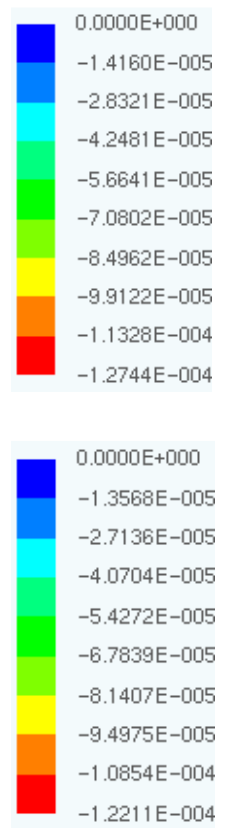

$-1.2211 \mathrm{E}-004$

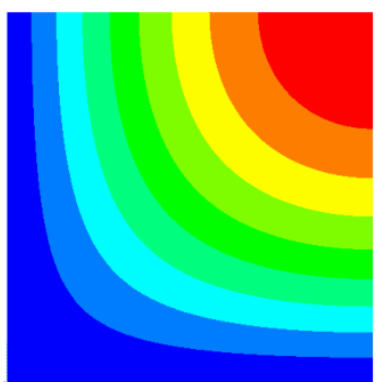

(a)

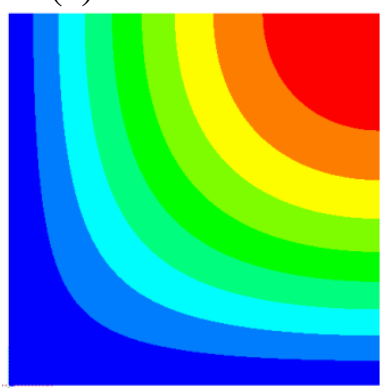

(c)
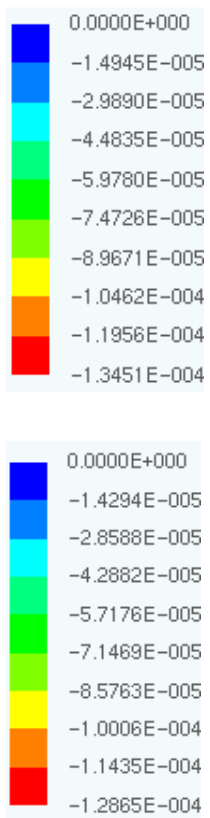

$-1.2865 \mathrm{E}-004$

Figura 75 - Deslocamentos transversais para um quarto do problema: (a) placa reforçada em uma direção laminada equivalente; (b) placa reforçada em uma direção com fibras; (c) placa reforçada em duas direções laminada equivalente; (d) placa reforçada em duas direções com fibras.

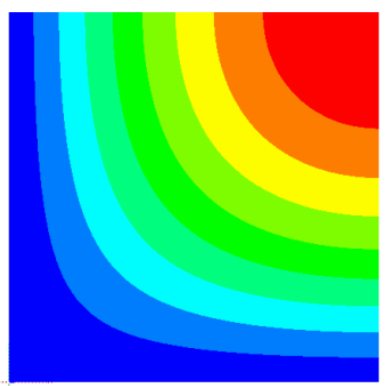

(b)

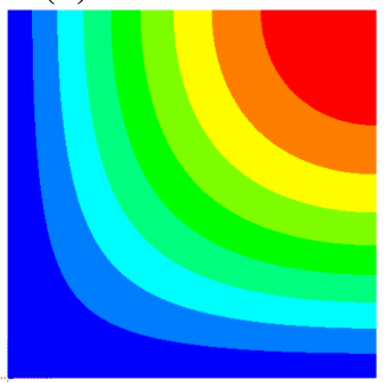

(d)

É possível observar da Tabela 8 que os resultados obtidos estão conforme esperado, ou seja, os deslocamentos transversais máximos obtidos para a placa reforçada em uma direção, para ambas as formulações, são menores que aqueles obtidos para a placa homogênea e são maiores que aqueles obtidos para a placa reforçada em duas direções. Além disso, é possível observar que as soluções obtidas com formulação introduzida neste trabalho são menores que aquelas obtidas com a formulação de referência, destacando que com a formulação 
introduzida neste trabalho será possível, em trabalhos futuros, determinar as tensões de contato entre as fibras e a matriz.

Tabela 8 - Valores numéricos para o deslocamento transversal máximo em (m).

\begin{tabular}{|c|c|c|c|c|}
\hline \multirow{2}{*}{$\begin{array}{c}\text { PLACA } \\
\text { HOMOGÊNEA }\end{array}$} & \multicolumn{2}{|c|}{ REFORÇADA EM 1 DIREÇÃO } & \multicolumn{2}{|c|}{ REFORÇADA EM 2 DIREÇÕES } \\
\hline & $\begin{array}{c}\text { LAMINADA } \\
\text { EQUIVALENTE }\end{array}$ & COM FIBRAS & $\begin{array}{c}\text { LAMINADA } \\
\text { EQUIVALENTE }\end{array}$ & COM FIBRAS \\
\hline$-1,4051 \times 10^{-4}$ & $-1,2744 \times 10^{-4}$ & $-1,3451 \times 10^{-4}$ & $-1,2211 \times 10^{-4}$ & $-1,2865 \times 10^{-4}$ \\
\hline
\end{tabular}

Outros valores para o módulo de elasticidade, coeficiente de Poisson e módulo de elasticidade transversal foram testados e os resultados obtidos para o deslocamento transversal máximo da placa podem ser visualizados na Figura 76.

O objetivo de testar diferentes valores de módulo de elasticidade, coeficiente de Poisson e módulo de elasticidade transversal é destacar a importância de se conhecer os parâmetros que descrevem corretamente as propriedades do meio, principalmente o módulo de elasticidade transversal visto que este problema se mostrou bastante sensível a este parâmetro. 


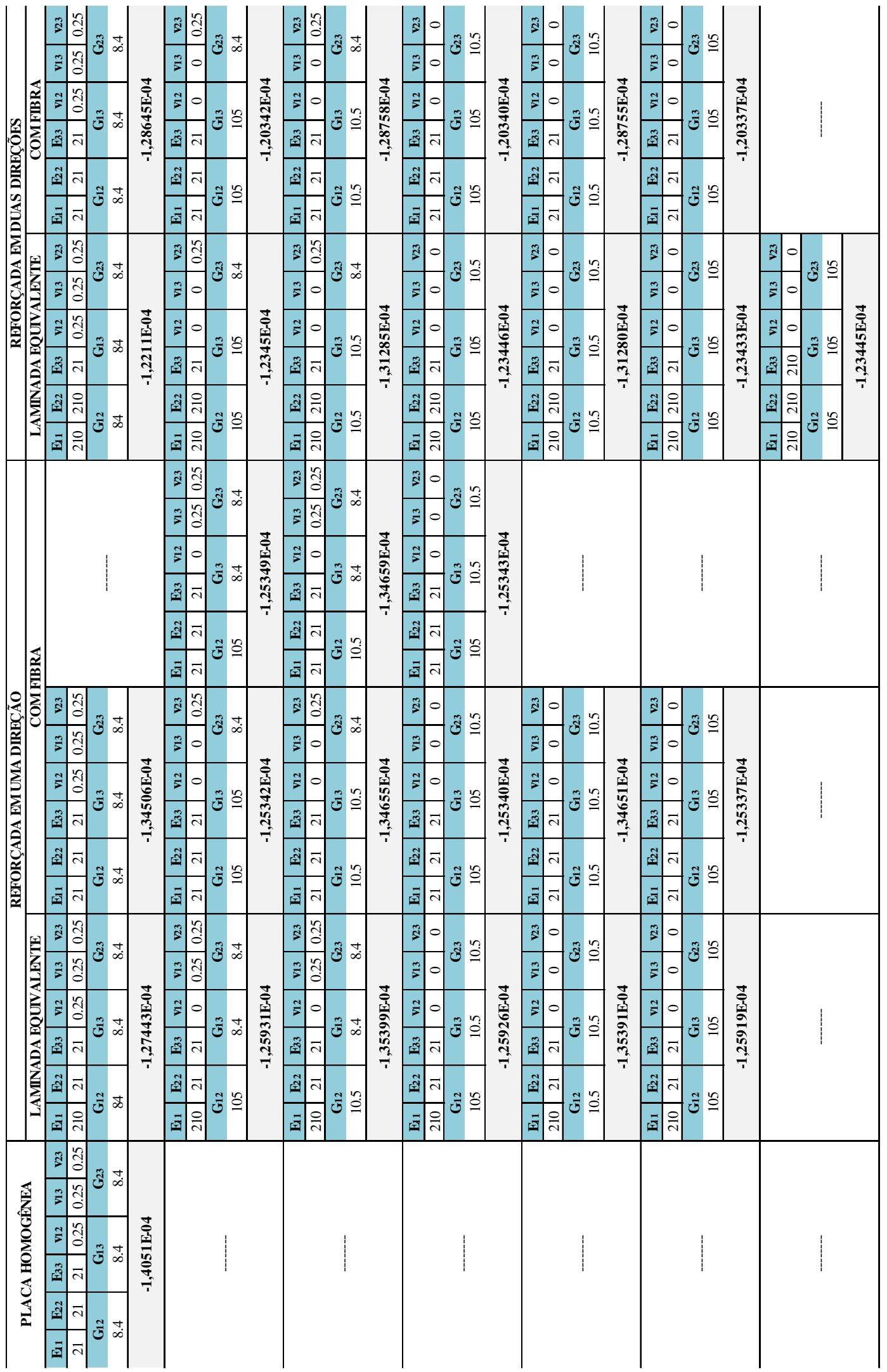

Figura 76 - Deslocamento transversal máximo para diferentes valores de módulo de elasticidade, coeficiente de Poisson e módulo de elasticidade transversal. 


\subsubsection{EXEMPLO 3: CASCA CILÍNDRICA LAMINADA REFORÇADA COM FIBRAS}

Neste exemplo, um cilindro laminado ortotrópico é analisado. A geometria do problema, extraída de Sansour e Kollmann (2000), pode ser visualizada na Figura 77.

Destaca-se que este exemplo foi utilizado por Coda, Paccola e Sampaio (2013) para validar a formulação de casca laminada utilizada aqui como solução de referência para os resultados obtidos com a formulação introduzida neste trabalho. Coda, Paccola e Sampaio (2013) compararam sua solução com uma solução analítica para cascas laminadas cilíndricas ortotrópicas em pequenos deslocamentos apresentada por Jones (2002). Coda, Paccola e Sampaio (2013) compararam os arranjos $0^{\circ} / 0^{\circ} / 0^{\circ}, 90^{\circ} / 90^{\circ} / 90^{\circ}, 0^{\circ} / 90^{\circ} / 0^{\circ}$ e o caso isotrópico homogêneo com os resultados apresentados por Jones (2002) e apresentaram um quarto arranjo $90^{\circ} / 0^{\circ} / 90^{\circ}$, para demonstrar que a formulação introduzida para cascas laminadas ortotrópicas não apresentava travamento.

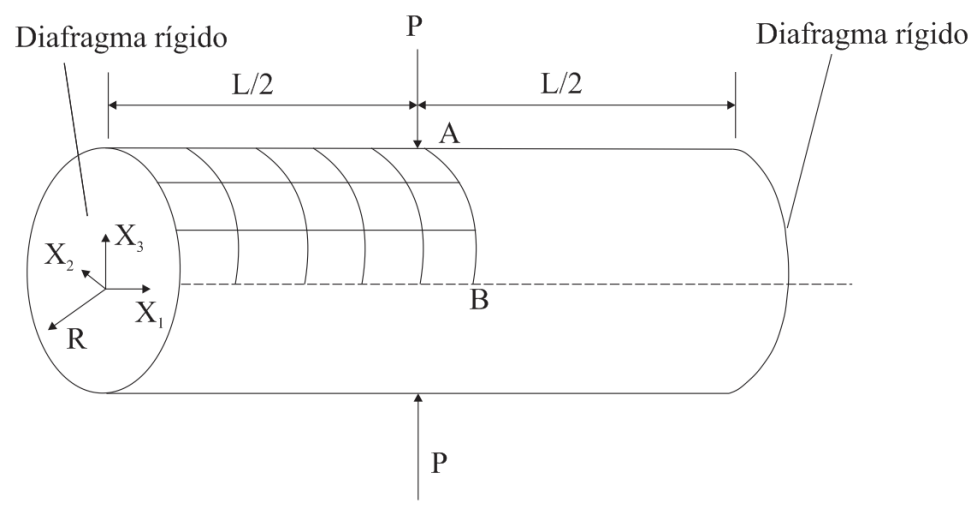

Figura 77 - Cilindro laminado comprimido. Fonte: (SANSOUR; KOLLMANN, 2000).

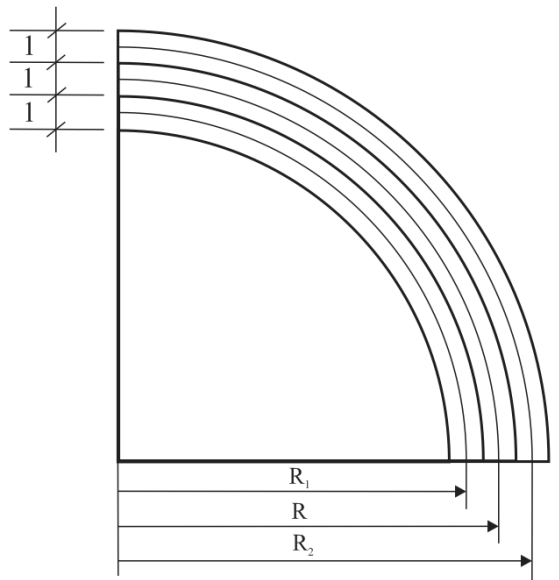

Figura 78 - Detalhe de 1/4 da seção transversal do cilindro laminado. 
Conforme foi comentado por Coda, Paccola e Sampaio (2013), uma vez que a metodologia utilizada para o desenvolvimento do elemento de casca proposto pelos autores é diferente daquela da solução de referência, analítica para pequenos deslocamentos, os valores convergentes devem se aproximar das soluções analíticas, mas certa diferença entre os resultados é esperada. De fato isto foi observado pelos autores que concluíram que a formulação se comporta de forma satisfatória. Lembra-se que na formulação apresentada por Coda, Paccola e Sampaio (2013), a relação constitutiva elástica é completa e a cinemática adotada considera a variação da espessura da casca.

Desta forma, os quatro arranjos testados por Coda, Paccola e Sampaio (2013) são aqui utilizados para analisar o comportamento da formulação de casca laminada considerando, agora, a discretização das fibras.

Como os autores compararam as soluções obtidas com outras de referência apresentadas por Jones (2002), estas não são aqui reescritas e apenas os resultados obtidos com a formulação de Coda, Paccola e Sampaio (2013) são utilizados como referência. Além disto, considerando que Coda, Paccola e Sampaio (2013) testaram diferentes malhas para analisar a convergência da solução do problema, somente a malha 20x20 mostrada na Figura 79 para $1 / 8$ do cilindro, definida como aceitável pelos autores, foi utilizada.

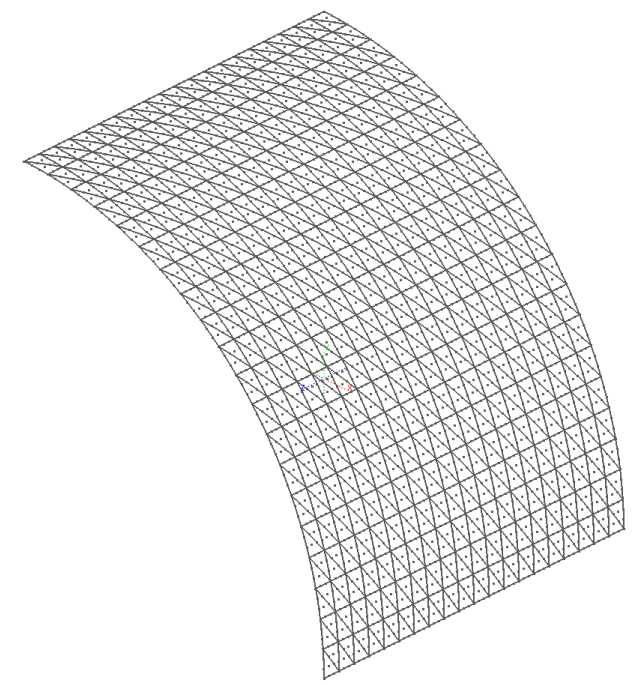

Figura 79 - Discretização da superfície de referência da matriz: 20x20 - 800 elementos com aproximação cúbica para as variáveis totalizando 3721 nós e 26047 graus de liberdade.

Da mesma forma como na referência original, as dimensões e outras propriedades físicas do problema não possuem unidades. Assim, os valores da geometria são: L $=600$, $\mathrm{R}=300$ e $\mathrm{h}=3,0$ dividida em 3 lâminas de espessura 1 cada. Nos arranjos considerados, 
$0^{\circ} / 0^{\circ} / 0^{\circ}, 90^{\circ} / 90^{\circ} / 90^{\circ}, 0^{\circ} / 90^{\circ} / 0^{\circ}$ e $90^{\circ} / 0^{\circ} / 90^{\circ}, 0^{\circ}$ corresponde à direção longitudinal de orientação das fibras e $90^{\circ}$ à direção circunferencial. As propriedades físicas adotadas na análise de cada um dos arranjos são especificadas na Tabela 9 e seguem os mesmos valores utilizados pela referência original. Os resultados obtidos para estas análises são designados como solução de referência laminada equivalente.

Tabela 9 - Dados utilizados para a modelagem do problema laminado equivalente com (E)x $10^{6}$ e $(\mathrm{G}) \times 10^{6}$.

\begin{tabular}{|c|c|c|c|c|c|c|c|c|c|c|}
\hline \multirow{3}{*}{ ARRANJO } & \multirow{3}{*}{ LÂMINA } & \multicolumn{9}{|c|}{ MATRIZ } \\
\hline & & \multicolumn{3}{|c|}{ E } & \multicolumn{3}{|c|}{$v$} & \multicolumn{3}{|c|}{$\mathbf{G}$} \\
\hline & & $\overline{\mathbf{E}_{1}}$ & $\mathbf{E}_{2}$ & $\overline{E_{3}}$ & $v_{12}$ & $v_{13}$ & $\mathrm{~V}_{23}$ & $\overline{G_{12}}$ & $\overline{\mathbf{G}_{13}}$ & $\mathbf{G}_{23}$ \\
\hline \multirow{3}{*}{$0^{\circ} / 0^{\circ} / 0^{\circ}$} & 1 & 40 & 1 & 1 & 0,25 & 0,25 & 0 & 0,6 & 0,6 & 0.5 \\
\hline & 2 & 40 & 1 & 1 & 0,25 & 0,25 & 0 & 0,6 & 0,6 & 0,5 \\
\hline & 3 & 40 & 1 & 1 & 0,25 & 0,25 & 0 & 0,6 & 0,6 & 0,5 \\
\hline \multirow{3}{*}{$90^{\circ} / 90^{\circ} / 90^{\circ}$} & 1 & 1 & 40 & 1 & 0,25 & 0 & 0,25 & 0,6 & 0,5 & 0,6 \\
\hline & 2 & 1 & 40 & 1 & 0,25 & 0 & 0,25 & 0,6 & 0,5 & 0,6 \\
\hline & 4 & 1 & 40 & 1 & 0,25 & 0 & 0,25 & 0,6 & 0,5 & 0,6 \\
\hline \multirow{3}{*}{$0^{\circ} / 90^{\circ} / 0^{\circ}$} & 1 & 40 & 1 & 1 & 0,25 & 0,25 & 0 & 0,6 & 0,6 & 0,5 \\
\hline & 2 & 1 & 40 & 1 & 0,25 & 0 & 0,25 & 0,6 & 0,5 & 0,6 \\
\hline & 3 & 40 & 1 & 1 & 0,25 & 0,25 & 0 & 0,6 & 0,6 & 0,5 \\
\hline \multirow{3}{*}{$90^{\circ} / 0^{\circ} / 90^{\circ}$} & 1 & 1 & 40 & 1 & 0,25 & 0 & 0,25 & 0,6 & 0,5 & 0,6 \\
\hline & 2 & 40 & 1 & 1 & 0,25 & 0,25 & 0 & 0,6 & 0,6 & 0,5 \\
\hline & 3 & 1 & 40 & 1 & 0,25 & 0 & 0,25 & 0,6 & 0,5 & 0,6 \\
\hline
\end{tabular}

\section{$\mathbf{0}^{\circ} / \mathbf{0}^{\%} / \mathbf{0}^{\circ}$}
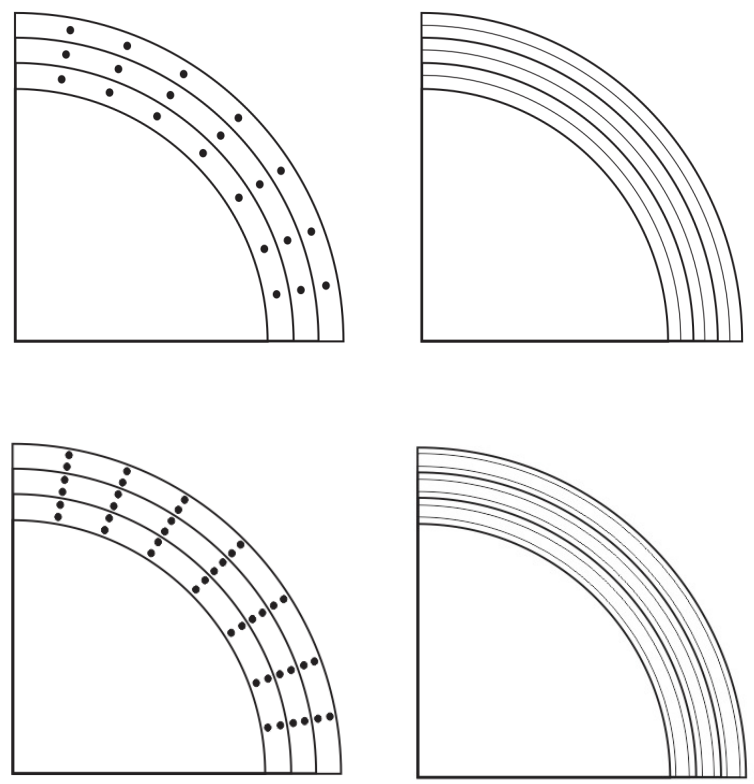

$90^{\circ} / 90^{\circ} / 90^{\circ}$

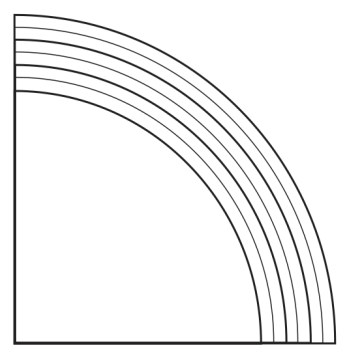

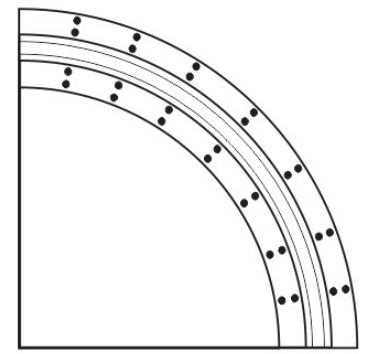

(a)

$0^{\circ} / 90^{\circ} / 0^{\circ}$

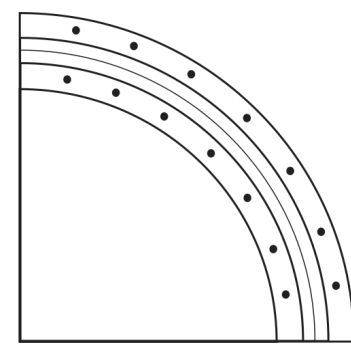

(b)

Figura 80 - Arranjos fibra-matriz considerados: $0^{\circ}$ corresponde à direção longitudinal e $90^{\circ}$ à direção circunferencial.

Para o problema com fibras, duas formas de disposição destas nas lâminas foram consideradas. Na primeira, as fibras estão posicionadas na superfície média de cada lâmina 
(Figura 80a), ou seja, em $R_{1}=299, \quad R=300$ e $R=301$ na Figura 78. Na segunda, duas camadas de fibras localizadas a $\pm 0,25$ da superfície média de cada lâmina foram consideradas (Figura 80b). Em ambos os casos de análises de fibras imersas, o material da matriz foi considerado isotrópico homogêneo. A Tabela 10 mostra os dados adotados para a matriz e o número de fibras necessários em cada lâmina para aproximar o módulo de elasticidade do problema equivalente quando as fibras estão localizadas na linha média da lâmina. Para o arranjo com duas camadas de fibras por lâmina, adotou-se $E_{\mathrm{f}}=426 \times 10^{6} \mathrm{e}$ $\mathrm{A}_{\mathrm{f}}=0,457$, lembrando-se que neste caso, as duas camadas de fibras por lâmina foram dispostas a $\pm 0,25$ da superfície média da lâmina considerada. Os demais dados são os mesmos da Tabela 10.

Para discretizar as fibras, foram utilizados elementos curvos com aproximação cúbica para as variáveis e cada fibra longa, em cada uma das camadas consideradas, foi dividida em 40 elementos finitos. O número total de fibras para cada arranjo para o caso de duas camadas de fibra por lâmina é mostrado na Tabela 11. As fibras foram igualmente espaçadas e dispostas a 3 unidades de comprimento das bordas para que uma fibra inteira não coincidisse com as extremidades da malha da matriz. Já as extremidades livres das fibras coincidem com a malha da matriz em todos os casos. $\mathrm{O}$ aspecto da discretização das fibras para os quatro arranjos considerados podem ser visualizado na Figura 81.

Da mesma forma como feito por Coda, Paccola e Sampaio (2013), a carga aplicada é suficientemente pequena, $\mathrm{P}=2,5 \times 10^{-7}$, para que seja possível a comparação com resultados em pequenos deslocamentos.

A Figura 82 mostra as configurações finais deformadas de 1/8 do cilindro, obtidas com a formulação de referência, casca laminada equivalente, e com a formulação proposta neste trabalho para os quatro arranjos testados. Os resultados exibidos na Figura 82b são aqueles obtidos para o caso em que se consideram duas camadas de fibras por lâmina.

A Tabela 12 compara o deslocamento na direção de P obtido no ponto de aplicação da carga $\mathrm{P}$ para os diferentes arranjos considerados, tanto para a fibra na linha média quanto para fibras em duas camadas, com aqueles obtidos pela formulação de referência. Aqui, o deslocamento foi designado pela letra $W$ e $n=4$ é a divisão do carregamento devido à discretização adotada, 1/8 do cilindro. O deslocamento final a ser comparado é obtido pela relação $\mathrm{EhW} / \mathrm{nP}$. 
Tabela 10 - Dados utilizados para a modelagem do problema com fibras com (E)x $10^{6}$ e $(\mathrm{G}) \times 10^{6}$.

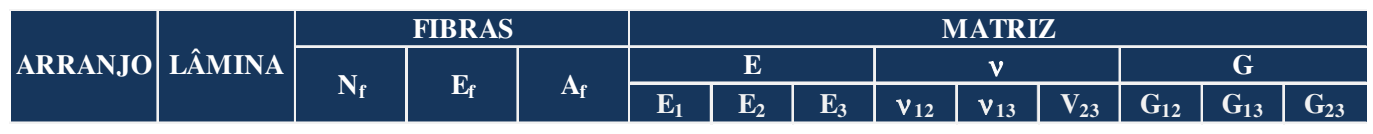

\begin{tabular}{|c|c|c|c|c|c|c|c|c|c|c|c|c|c|}
\hline \multirow{3}{*}{$0^{\circ} / 0^{\circ} / 0^{\circ}$} & 1 & 47 & 39 & 1 & 1 & 1 & 1 & 0,25 & 0,25 & 0 & 0,4 & 0,4 & 0,5 \\
\hline & 2 & 47 & 39 & 1 & 1 & 1 & 1 & 0,25 & 0,25 & 0 & 0,4 & 0,4 & 0,5 \\
\hline & 3 & 47 & 39 & 1 & 1 & 1 & 1 & 0,25 & 0,25 & 0 & 0,4 & 0,4 & 0,5 \\
\hline \multirow{3}{*}{$90^{\circ} / 90^{\circ} / 90^{\circ}$} & 1 & 30 & 39 & 1 & 1 & 1 & 1 & 0,25 & 0 & 0,25 & 0,4 & 0,5 & 0,4 \\
\hline & 2 & 30 & 39 & 1 & 1 & 1 & 1 & 0,25 & 0 & 0,25 & 0,4 & 0,5 & 0,4 \\
\hline & 4 & 30 & 39 & 1 & 1 & 1 & 1 & 0,25 & 0 & 0,25 & 0,4 & 0,5 & 0,4 \\
\hline \multirow{3}{*}{$0^{\circ} / 90^{\circ} / 0^{\circ}$} & 1 & 47 & 39 & 1 & 1 & 1 & 1 & 0,25 & 0,25 & 0 & 0,4 & 0,4 & 0,5 \\
\hline & 2 & 30 & 39 & 1 & 1 & 1 & 1 & 0,25 & 0 & 0,25 & 0,4 & 0,5 & 0,4 \\
\hline & 3 & 47 & 39 & 1 & 1 & 1 & 1 & 0,25 & 0,25 & 0 & 0,4 & 0,4 & 0,5 \\
\hline \multirow{3}{*}{$90^{\circ} / 0^{\circ} / 90^{\circ}$} & 1 & 30 & 39 & 1 & 1 & 1 & 1 & 0,25 & 0 & 0,25 & 0,4 & 0,5 & 0,4 \\
\hline & 2 & 47 & 39 & 1 & 1 & 1 & 1 & 0,25 & 0,25 & 0 & 0,4 & 0,4 & 0,5 \\
\hline & 3 & 30 & 39 & 1 & 1 & 1 & 1 & 0,25 & 0 & 0,25 & 0,4 & 0,5 & 0,4 \\
\hline
\end{tabular}

Tabela 11 - Número total de nós de fibra longa para o arranjo com duas camadas de fibra por lâmina.

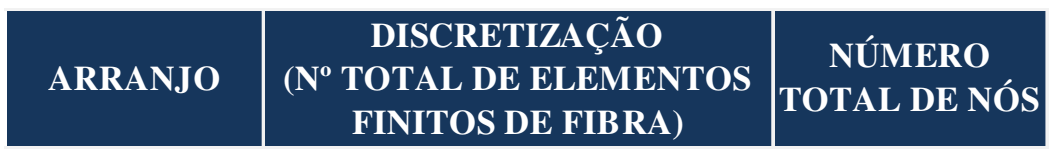

\begin{tabular}{|c|c|c|}
\hline $0^{\circ} / 0^{\circ} / 0^{\circ}$ & 11280 & 34122 \\
\hline $90^{\circ} / 90^{\circ} / 90^{\circ}$ & 7200 & 21780 \\
\hline $0^{\circ} / 90^{\circ} / 0^{\circ}$ & 9920 & 30008 \\
\hline $90^{\circ} / 0^{\circ} / 90^{\circ}$ & 8560 & 25894 \\
\hline
\end{tabular}

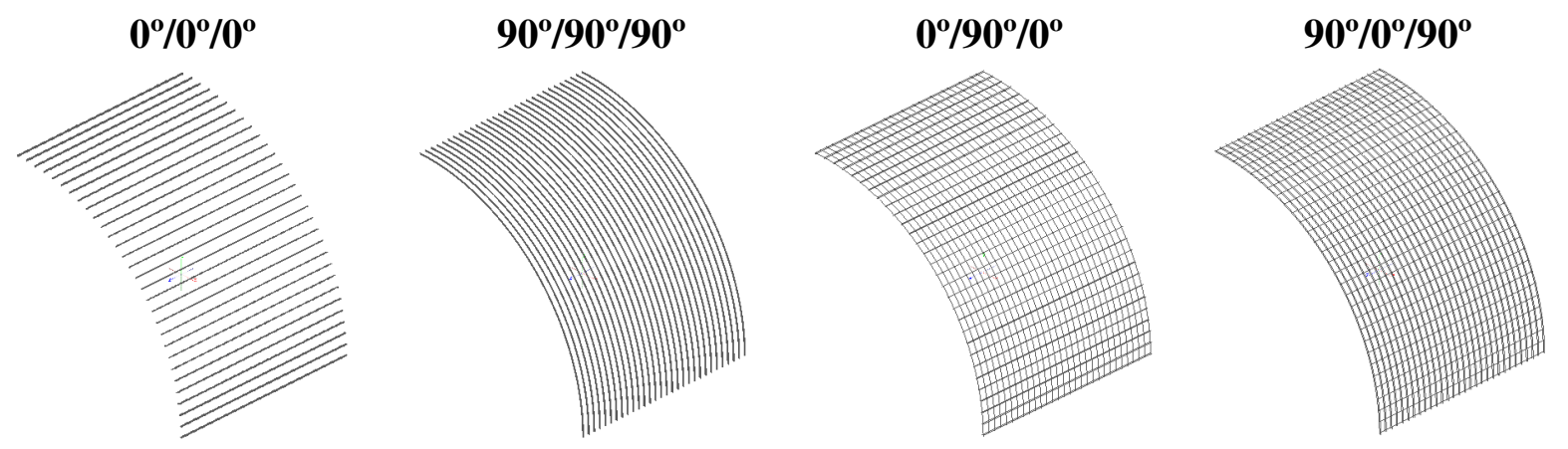

Figura 81 - Discretização das fibras para os diferentes arranjos testados.

Tabela 12 - Deslocamento total na direção de P para o ponto de aplicação da carga P.

\begin{tabular}{|c|c|c|c|c|c|c|c|c|}
\hline \multirow{4}{*}{ ARRANJO } & \multirow{2}{*}{\multicolumn{2}{|c|}{ LAMINADA EQUIVALENTE }} & \multicolumn{6}{|c|}{ LAMINADA COM FIBRAS } \\
\hline & & & \multicolumn{3}{|c|}{1 CAMADA DE FIBRAS POR LÂMINA } & \multicolumn{3}{|c|}{2 CAMADAS DE FIBRAS POR LÂMINA } \\
\hline & \multicolumn{2}{|c|}{ (REFERÊNCIA) } & \multirow{2}{*}{ W } & \multirow{2}{*}{ EhW/nP } & \multirow{2}{*}{$\begin{array}{c}\text { DESVIO } \\
\text { RELATIVO (\%) }\end{array}$} & \multirow{2}{*}{ W } & \multirow{2}{*}{ EhW/nP } & \multirow{2}{*}{$\begin{array}{c}\text { DESVIO } \\
\text { RELATIVO (\% }\end{array}$} \\
\hline & W & EhW/nP & & & & & & \\
\hline
\end{tabular}

\begin{tabular}{|c|c|c|c|c|c|c|c|c|}
\hline $0^{\circ} / 0^{\circ} / 0^{\circ}$ & 2,3954E-05 & 2874,48 & $2,6876 \mathrm{E}-05$ & 3225,12 & 12,198 & $2,6580 \mathrm{E}-05$ & 3189,6 & 10,96 \\
\hline $90^{\circ} / 90^{\circ} / 90^{\circ}$ & 8,2901E-06 & 994,812 & $9,3320 \mathrm{E}-06$ & 1119,84 & 12,568 & 8,9692E-06 & 1076,304 & 8,19 \\
\hline $0^{\circ} / 90^{\circ} / 0^{\circ}$ & $1,5902 \mathrm{E}-05$ & 1908,24 & 2,6083E-05 & 3129,96 & 64,023 & $1,9047 \mathrm{E}-05$ & 2285,64 & 19,78 \\
\hline $90^{\circ} / 0^{\circ} / 90^{\circ}$ & $6,4651 \mathrm{E}-06$ & 775,812 & $7,9080 \mathrm{E}-06$ & 948,96 & 22,318 & $7,2907 \mathrm{E}-06$ & 874,884 & 12,77 \\
\hline
\end{tabular}




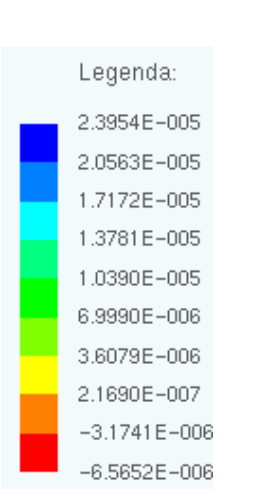

(a)

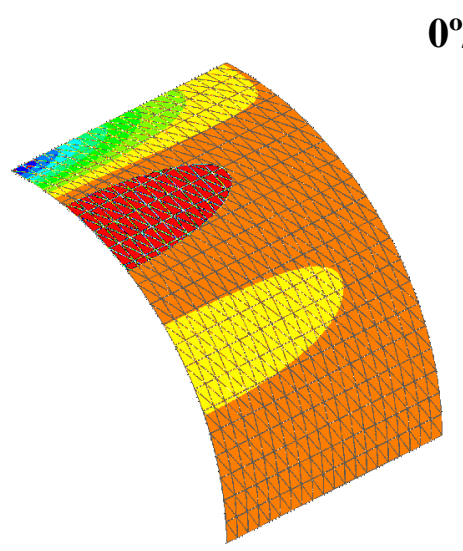

$90^{\circ} / 90^{\circ} / 90^{\circ}$

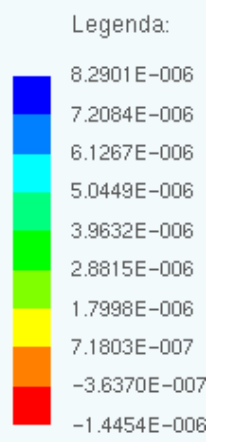

(a)

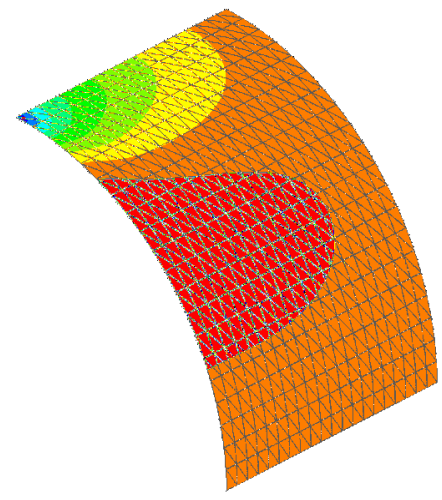

$0^{\circ} / 90^{\circ} / 0^{\circ}$

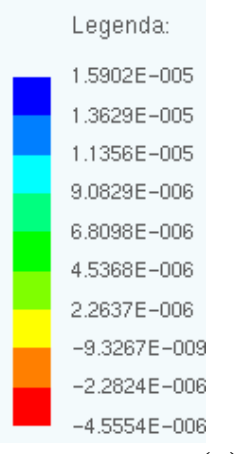

(a)

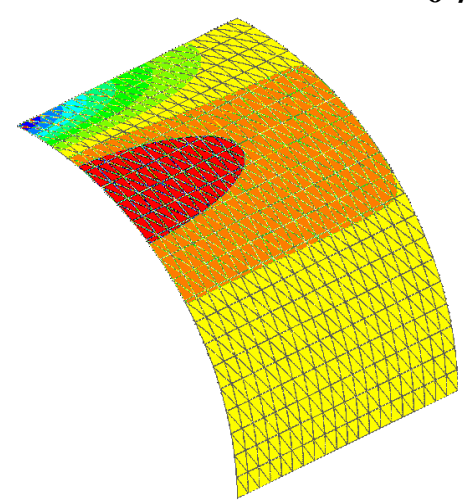

$90^{\circ} / 0^{\circ} / 90^{\circ}$

\begin{tabular}{|c|}
\hline Legenda: \\
\hline $6.4651 \mathrm{E}-006$ \\
\hline $5.6196 \mathrm{E}-006$ \\
\hline $4.7741 \mathrm{E}-006$ \\
\hline $3.9287 \mathrm{E}-006$ \\
\hline $3.0832 E-006$ \\
\hline $2.2377 \mathrm{E}-006$ \\
\hline $1.3922 \mathrm{E}-006$ \\
\hline $5.4678 \mathrm{E}-007$ \\
\hline$-2.9869 \mathrm{E}-007$ \\
\hline$-1.1442 E-006$ \\
\hline
\end{tabular}

(a)

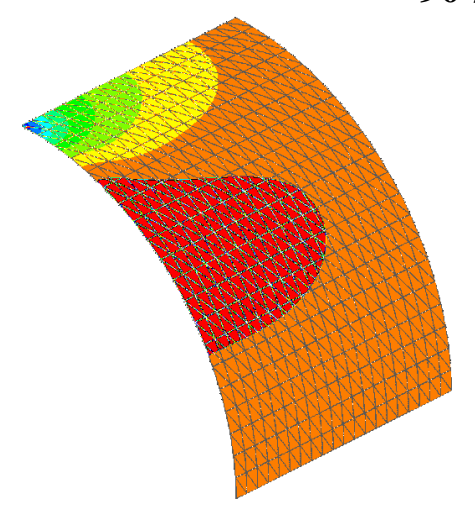

$0^{\circ} / 0^{\circ} / 0^{\circ}$
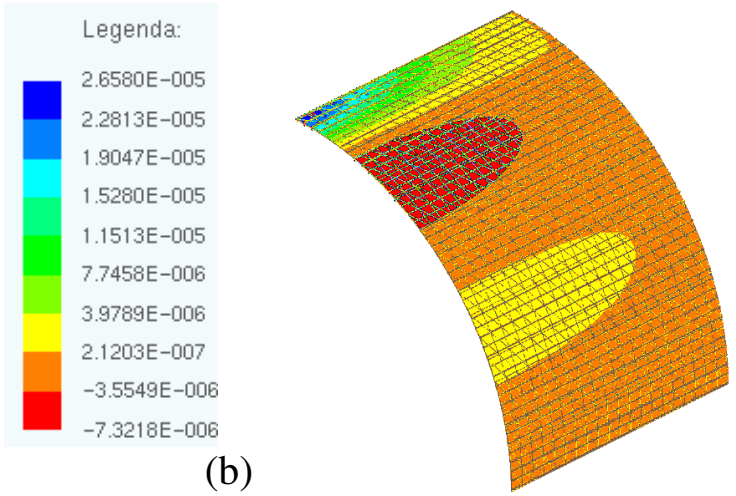

(b)

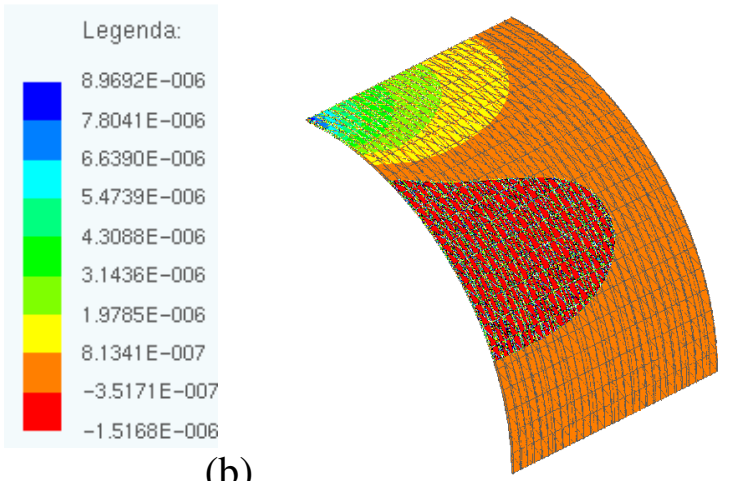

(b)

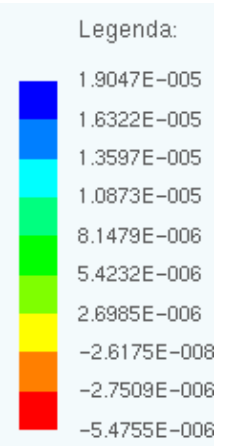

(b)
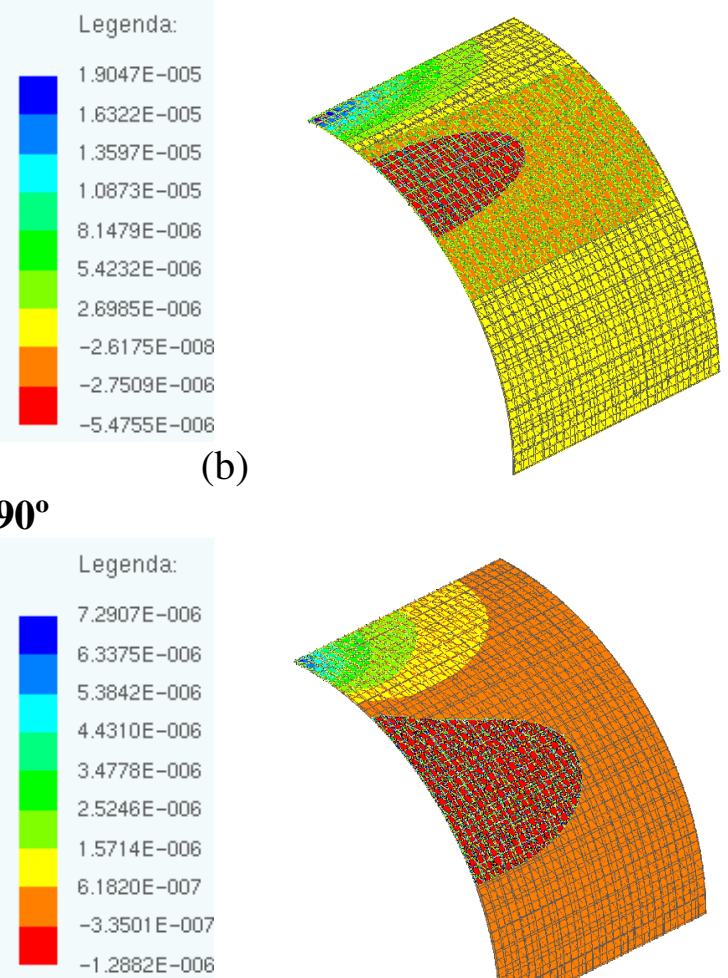

(b)

Figura 82 - Deslocamentos na direção de P de 1/8 do cilindro para os diferentes arranjos testados: (a) laminada equivalente; (b) laminada com fibras. 
É possível observar a partir dos resultados obtidos que a formulação introduzida neste trabalho fornece valores maiores que a solução de referência em todos os casos analisados e para os dados considerados. Analisando-se primeiramente o caso em que as fibras são dispostas na linha média das camadas, observa-se da Tabela 12 um grande desvio entre as soluções para os arranjos $0^{\circ} / 90^{\circ} / 0^{\circ}$ e $90^{\circ} / 0^{\circ} / 90^{\circ}$. Isso ocorre devido à forma como as fibras foram dispostas nas camadas, na linha média, influenciando nas soluções quando a contribuição da inércia à flexão da lâmina central é determinante na constituição da inércia total da casca. Ao mudar a disposição das fibras na matriz, considerando-se duas fibras por camada e consequentemente afastando-as da linha média das camadas, observa-se, da Tabela 12, a diminuição nos desvios entre as soluções, principalmente para os arranjos $0^{\circ} / 90^{\circ} / 0^{\circ} \mathrm{e}$ $90^{\circ} / 0^{\circ} / 90^{\circ}$.

Acredita-se que à medida que o número de fibras utilizado na discretização aumenta e os parâmetros que descrevem corretamente as propriedades do meio são conhecidos, como por exemplo, o módulo de elasticidade transversal $\mathrm{G}$ para o qual este problema se mostra bastante sensível, o comportamento das soluções obtidas pela formulação proposta tende a ser semelhante ao apresentado pelo meio homogeneizado. De qualquer forma, por serem problemas diferentes não se espera equidade de resultados.

\subsubsection{CASCA LAMINADA REFORÇADA COM FIBRAS ALEATÓRIAS}

Para demonstrar as potencialidades da formulação, um caso qualitativo é apresentado. Nele, fibras curtas são espalhadas aleatoriamente nas lâminas da casca na taxa de $1 \%$ do volume do cilindro. Três comprimentos para as fibras foram considerados, $\mathrm{L}_{\mathrm{f}}=2,54, \mathrm{~L}_{\mathrm{f}}=5$ e $\mathrm{L}_{\mathrm{f}}=10$. Em todos os casos, o módulo de elasticidade e a área da seção transversal são dados, respectivamente, por $\mathrm{E}_{\mathrm{f}}=426 \times 10^{6}$ e $\mathrm{A}_{\mathrm{f}}=0,457$. Com estes dados, o número total de fibras com aproximação cúbica para as variáveis para cada caso considerado é dado, respectivamente, por 3568,1854 e 924 . As fibras foram espalhadas em seis camadas, duas por lâmina, a $\pm 0,25$ da superfície média da lâmina. A Tabela 13 mostra um quadro resumo dos dados adotados para as fibras e a Tabela 14 o número total de nós de fibra em cada discretização.

Primeiramente analisou-se o cilindro laminado sem a contribuição das fibras e em seguida considerou-se a presença das fibras. Os dados adotados na análise da matriz são 
mostrados na Tabela 15. As configurações finais deformadas para $1 / 8$ do cilindro correspondentes às análises realizadas podem ser visualizadas na Figura 83.

Tabela 13 - Dados das fibras aleatórias com (E)x10

\begin{tabular}{|c|c|c|c|c|c|c|c|c|c|c|}
\hline \multirow{3}{*}{ LÂMINA } & \multirow{3}{*}{ CAMADA } & \multicolumn{9}{|c|}{ FIBRAS ALEATÓRIAS } \\
\hline & & \multirow{2}{*}{$\mathbf{E}_{\mathbf{f}}$} & \multirow{2}{*}{$\mathbf{A}_{\mathbf{f}}$} & \multirow{2}{*}{$\xi_{3}$} & \multicolumn{2}{|c|}{ CASO 1} & \multicolumn{2}{|c|}{ CASO 2} & \multicolumn{2}{|c|}{ CASO 3} \\
\hline & & & & & $\mathbf{N}_{\mathbf{f}}$ & $\overline{\mathbf{L}_{\mathbf{f}}}$ & $\mathbf{N}_{\mathbf{f}}$ & $\overline{\mathbf{L}_{\mathbf{f}}}$ & $\mathbf{N}_{\mathbf{f}}$ & $\overline{\mathbf{L}_{\mathbf{f}}}$ \\
\hline \multirow{2}{*}{1} & 1 & 426 & 0,457 & +0.5 & 608 & 2,54 & 309 & 5 & 154 & 10 \\
\hline & 2 & 426 & 0,457 & -0.5 & 608 & 2,54 & 309 & 5 & 154 & 10 \\
\hline \multirow{2}{*}{2} & 1 & 426 & 0,457 & +0.5 & 608 & 2,54 & 309 & 5 & 154 & 10 \\
\hline & 2 & 426 & 0,457 & -0.5 & 608 & 2,54 & 309 & 5 & 154 & 10 \\
\hline \multirow{2}{*}{3} & 1 & 426 & 0,457 & +0.5 & 608 & 2,54 & 309 & 5 & 154 & 10 \\
\hline & 2 & 426 & 0,457 & -0.5 & 608 & 2,54 & 309 & 5 & 154 & 10 \\
\hline
\end{tabular}

Tabela 14 - Número total de nós de fibras aleatórias.

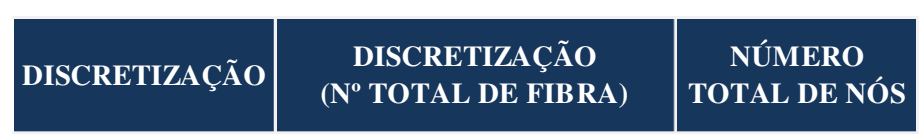

\begin{tabular}{ccc}
\hline CASO 1 & 3648 & 14592 \\
\hline CASO 2 & 1854 & 7416 \\
\hline CASO 3 & 924 & 3696 \\
\hline
\end{tabular}

Tabela 15 - Dados da matriz para análise do domínio com fibras aleatórias.

\begin{tabular}{|c|c|c|c|c|c|c|c|c|c|}
\hline \multirow{3}{*}{ LÂMINA } & \multicolumn{9}{|c|}{ MATRIZ } \\
\hline & \multicolumn{3}{|c|}{$\mathbf{E}$} & \multicolumn{3}{|c|}{$\mathrm{v}$} & \multicolumn{3}{|c|}{$\mathbf{G}$} \\
\hline & $E_{1}$ & $\mathbf{E}_{2}$ & $\mathbf{E}_{3}$ & $v_{12}$ & $v_{13}$ & $\mathbf{V}_{23}$ & $\mathbf{G}_{12}$ & $\mathbf{G}_{13}$ & $\mathbf{G}_{23}$ \\
\hline 1 & 1 & 1 & 1 & 0,25 & 0,25 & 0 & 0,4 & 0,4 & 0,5 \\
\hline 2 & 1 & 1 & 1 & 0,25 & 0,25 & 0 & 0,4 & 0,4 & 0,5 \\
\hline 3 & 1 & 1 & 1 & 0,25 & 0,25 & 0 & 0,4 & 0,4 & 0,5 \\
\hline
\end{tabular}

Tabela 16 - Deslocamentos na direção de P para o ponto de aplicação da carga P do cilindro reforçado com fibras aleatórias.

\begin{tabular}{|c|c|c|c|c|c|c|c|}
\hline \multirow{2}{*}{\multicolumn{2}{|c|}{ LAMINADA EQUIVALENTE }} & \multicolumn{6}{|c|}{ LAMINADA COM FIBRAS } \\
\hline & & \multicolumn{2}{|c|}{$L_{\mathrm{f}}=2,54$} & \multicolumn{2}{|c|}{$\mathbf{L}_{\mathrm{f}}=\mathbf{5}$} & \multicolumn{2}{|c|}{$L_{\mathrm{f}}=10$} \\
\hline $\mathbf{W}$ & EhW/nP & $\mathbf{W}$ & EhW/nP & $\mathbf{W}$ & EhW/nP & W & EhW/nP \\
\hline $5,6260 \mathrm{E}-05$ & 6751,20 & 4,3387E-05 & 5206,44 & $4,4032 \mathrm{E}-05$ & 5283,84 & $4,8015 \mathrm{E}-05$ & 5761,8 \\
\hline DESVIO REI & TIVO (\%) & & 22,881 & & 21,735 & & 14,655 \\
\hline
\end{tabular}


(a)

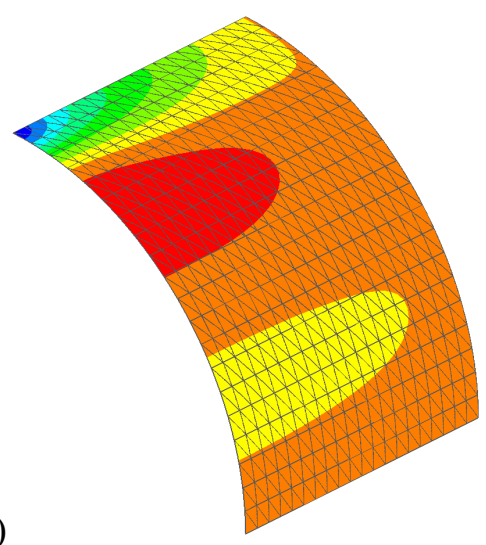

(b)

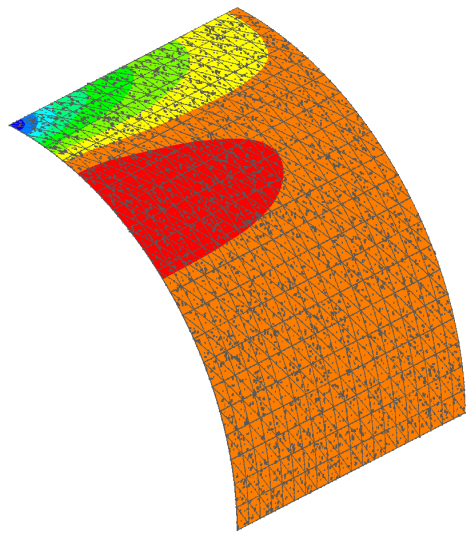

(c)

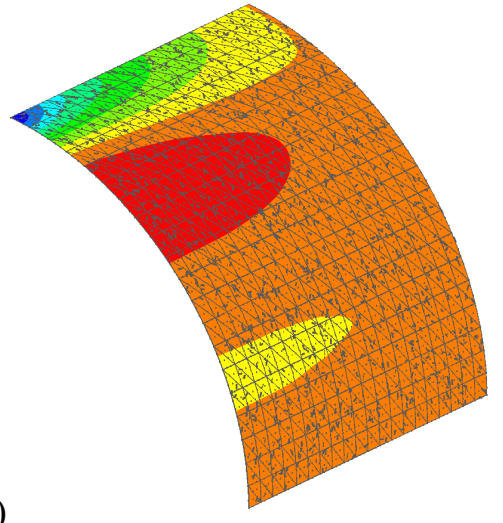

(d)

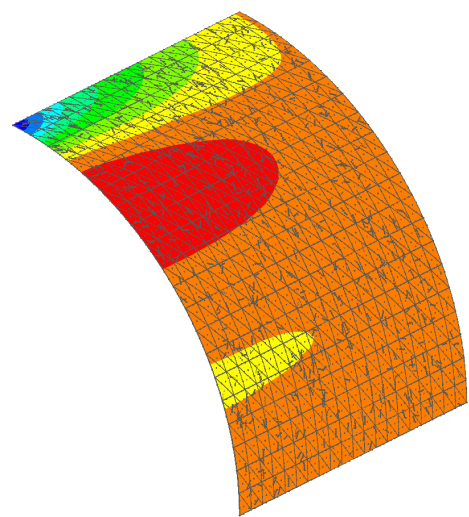

Legenda:

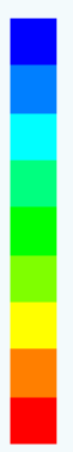

$5.6260 \mathrm{E}-005$

4.8274E-005

$4.0288 \mathrm{E}-005$

3.2302E-005

$2.4315 \mathrm{E}-005$

$1.6329 \mathrm{E}-005$

8.3429E-006

$3.5672 \mathrm{E}-007$

$-7.6295 \mathrm{E}-006$

$-1.5616 \mathrm{E}-005$

Legenda:

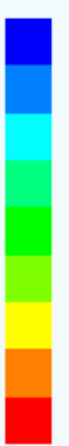

4.3387E-005

3.7335E-005

3.1283E-005

2.5230E-005

$1.9178 \mathrm{E}-005$

$1.3126 \mathrm{E}-005$

$7.0736 \mathrm{E}-006$

$1.0214 \mathrm{E}-006$

$-5.0309 \mathrm{E}-006$

$-1.1083 \mathrm{E}-005$

Legenda:

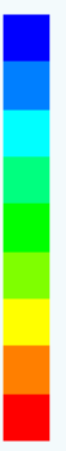

4.4032E-005

3.7807E-005

3.1583E-005

2.5358E-005

1.9133E-005

1.2908E-005

6.6837E-006

4.5894E-007

$-5.7658 \mathrm{E}-006$

$-1.1991 \mathrm{E}-005$

Legenda:

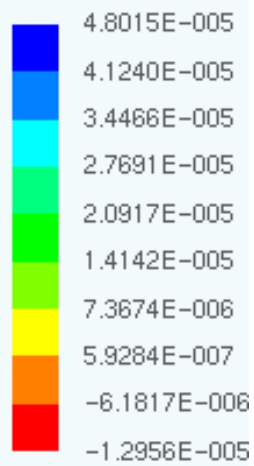

Figura 83 - Configurações finais deformadas de 1/8 do cilindro laminado: (a) sem fibras; (b) com 3568 fibras aleatórias; (c) com 1854 fibras aleatórias; (d) com 924 fibras aleatórias. 
Os valores obtidos pela relação $\mathrm{EhW} / \mathrm{nP}$, na direção de $\mathrm{P}$ para o ponto de aplicação da carga P para os casos analisados, bem como a diferença entre os deslocamentos obtidos para os casos com fibras em relação ao deslocamento obtido para o cilindro sem fibras são mostrados na Tabela 16.

Em todos os casos, a adição das fibras enrijeceu o conjunto, porém, observa-se que embora o volume de fibras considerado nos casos analisados seja o mesmo, $1 \%$ do volume do cilindro, os resultados obtidos para a discretização menos densa (Figura 83d) são maiores que aqueles obtidos para a discretização mais densa (Figura 83b). Detalhes sobre a geração das fibras aleatórias são apresentados no APÊNDICE C.

\subsubsection{CASCA LAMINADA REFORÇADA COM FIBRAS EM GRANDES DESLOCAMENTOS}

Para finalizar, realizou-se a análise em grandes deslocamentos dos arranjos $0^{\circ} / 0^{\circ} / 0^{\circ} \mathrm{e}$ $0^{\circ} / 90^{\circ} / 0^{\circ}$ pelas formulações laminada equivalente e laminada com fibras e do caso com fibras aleatórias. Neste caso, $\mathrm{P}=0,25$ foi aplicado em 50 passos de carga. Todos os demais dados utilizados na análise são os mesmos adotados para o caso linear.

A Figura 84 mostra as trajetórias de equilíbrio do ponto de aplicação da carga $\mathrm{P}$ para os arranjos $0^{\circ} / 0^{\circ} 0^{\circ}$ e $0^{\circ} / 90^{\circ} / 0^{\circ}$ obtidas com a formulação de referência e com a formulação introduzida neste trabalho. A Figura 85 mostra a trajetória de equilíbrio do ponto de aplicação da carga P para a casca sem fibras e com fibras aleatórias. A Figura 86 compara as trajetórias de equilíbrio da Figura 84 e da Figura 85 e a Figura 87 mostra as configurações finais deformadas dos casos analisados.

Da Figura 84 observa-se que os deslocamentos apresentados pela casca laminada com o arranjo $0^{\circ} / 0^{\circ} / 0^{\circ}$ são maiores que aqueles apresentados pelo arranjo $0^{\circ} / 90^{\circ} / 0^{\circ}$, tanto para a formulação laminada equivalente quanto para a formulação com fibras, e que em ambos os arranjos a casca com fibras modelada com a formulação introduzida neste trabalho apresenta maior deslocabilidade do que quando modelada com formulação laminada equivalente.

Destaca-se da Figura 85 a grande deslocabilidade da casca laminada reforçada com fibras aleatórias e como consequência disto a presença de alguns trechos que parecem indicar instabilidades na trajetória de equilíbrio deste caso. 


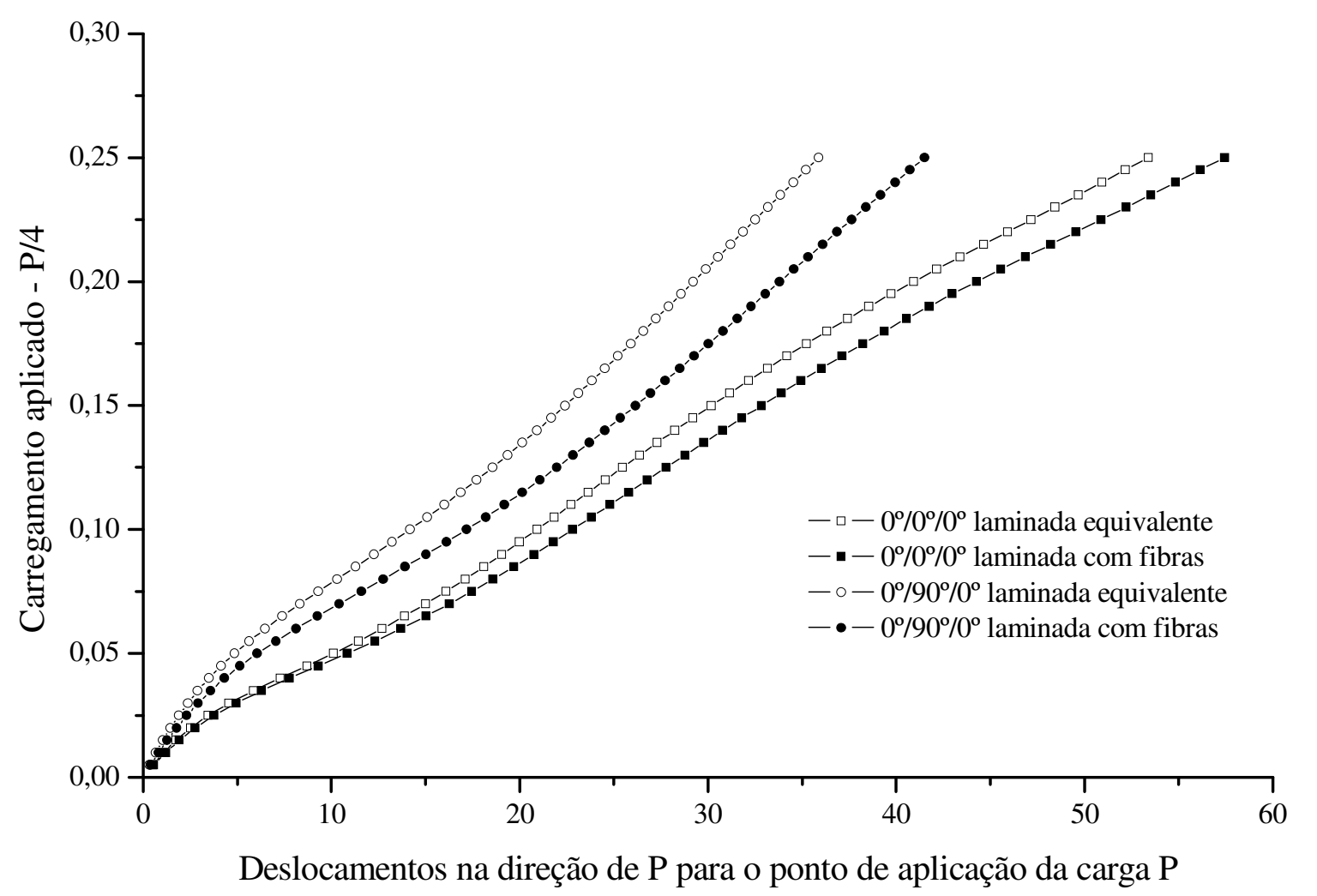

Figura 84 - Trajetória de equilíbrio do ponto de aplicação da carga P para os diferentes arranjos considerados.

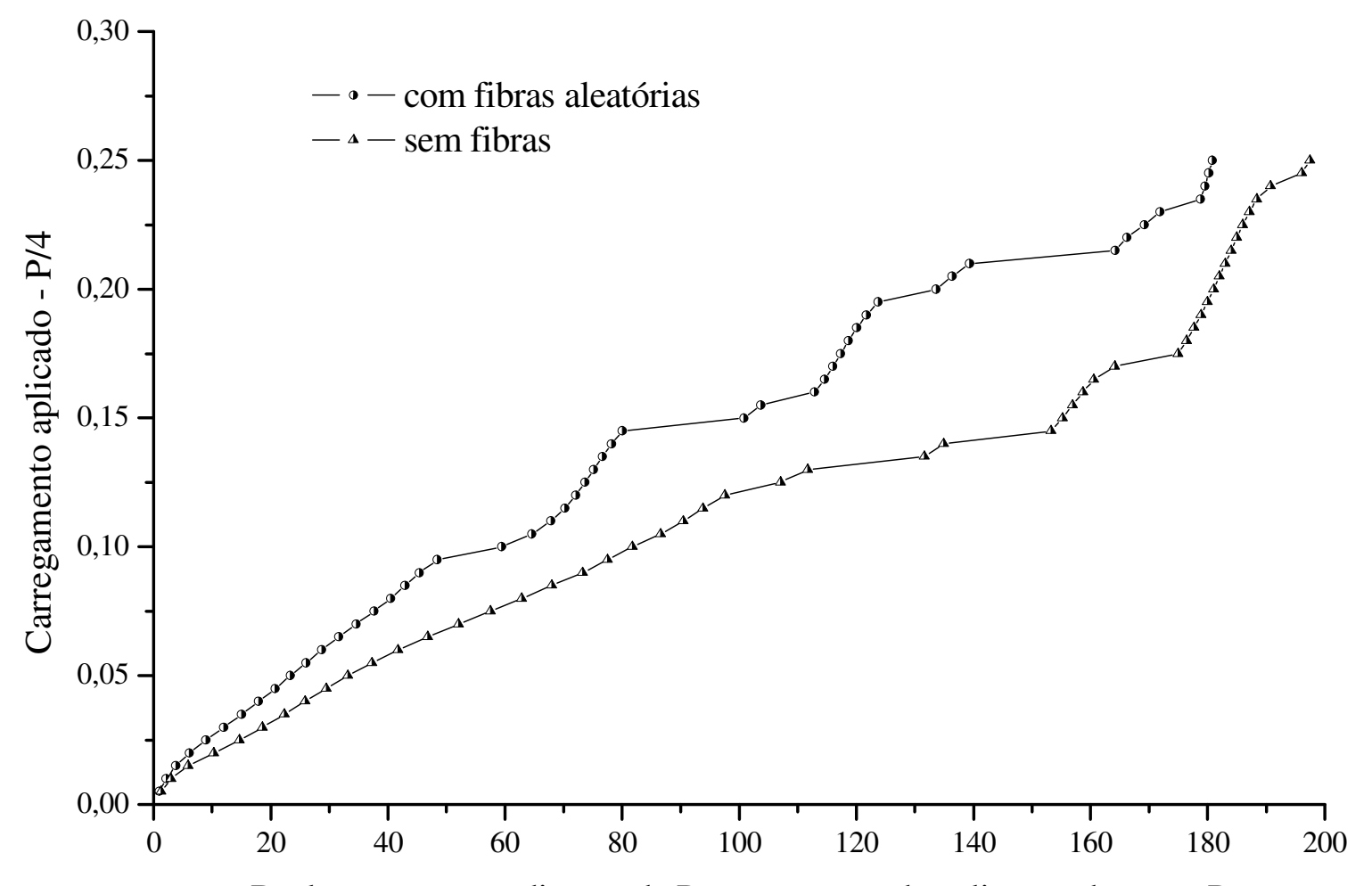

Deslocamentos na direção de $\mathrm{P}$ para o ponto de aplicação da carga $\mathrm{P}$

Figura 85 - Trajetória de equilíbrio do ponto de aplicação da carga P para a casca laminada reforçada com fibras aleatórias. 


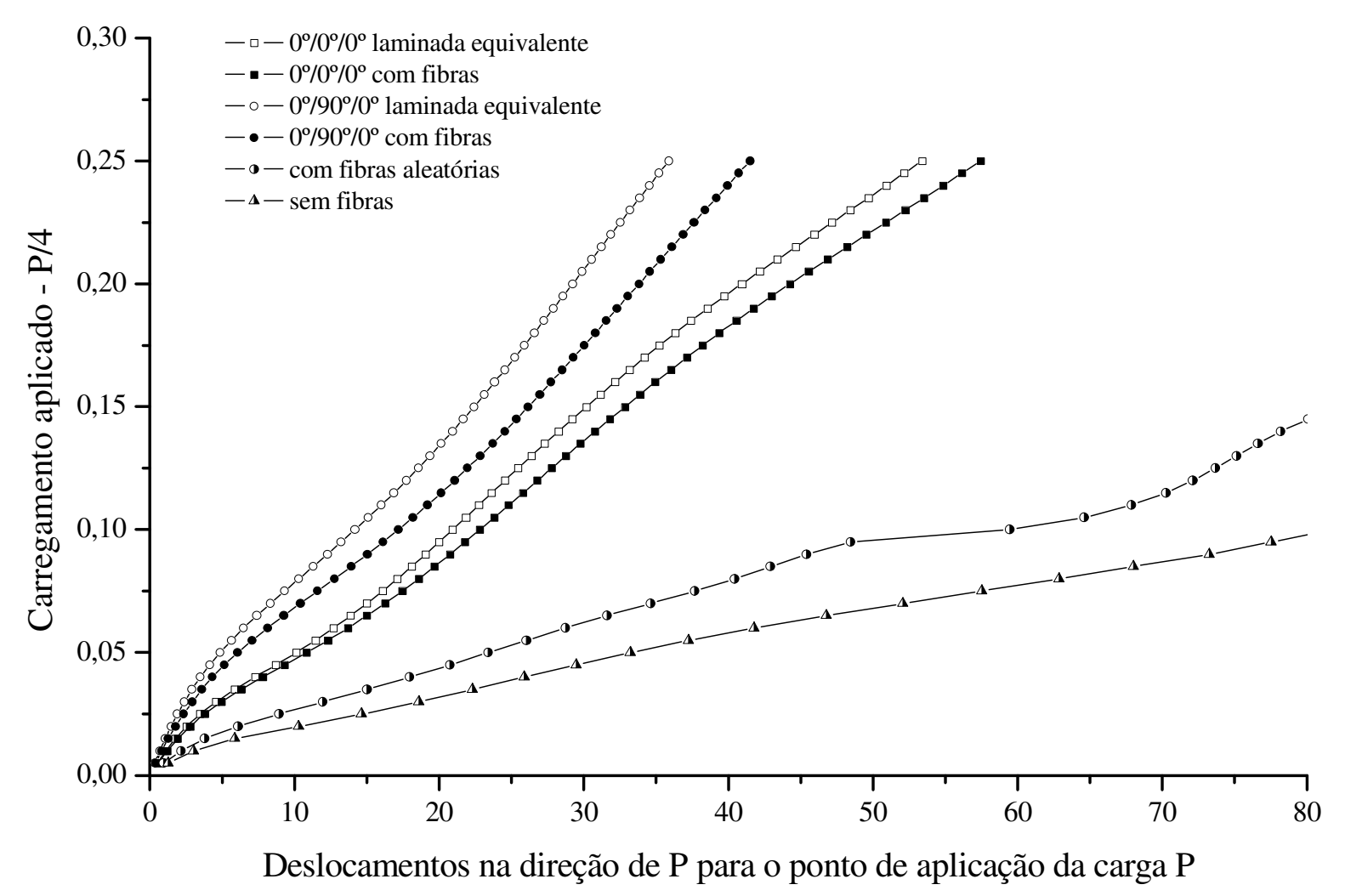

Figura 86 - Comparação entre as trajetórias de equilíbrio para os arranjos considerados.

A Figura 86 compara as trajetórias de equilíbrio da Figura 84 e da Figura 85 no intervalo 0 a 80 para os deslocamentos do ponto de aplicação da carga $P$.

Considerando a complexidade do problema estudado, as variáveis envolvidas na sua modelagem, os vários arranjos testados, a presença de lâminas, a modelagem com fibras aleatórias, a sensibilidade da solução aos parâmetros que descrevem as propriedades do material como, por exemplo, o módulo de elasticidade transversal, etc., é possível observar com base nos resultados obtidos, que a formulação introduzida neste trabalho é adequada para a análise de cascas laminadas reforçada com fibras.

Por fim, mostram-se na Figura 88 e na Figura 89 vistas ampliadas da configuração final deformada de $1 / 8$ do cilindro reforçado com fibras para os arranjos $0^{\circ} / 0^{\circ} / 0^{\circ}$ e $0^{\circ} / 90^{\circ} / 0^{\circ}$, respectivamente. A Figura 90 e a Figura 91 mostram vistas ampliadas de 1/8 do cilindro reforçado com fibras aleatórias. 

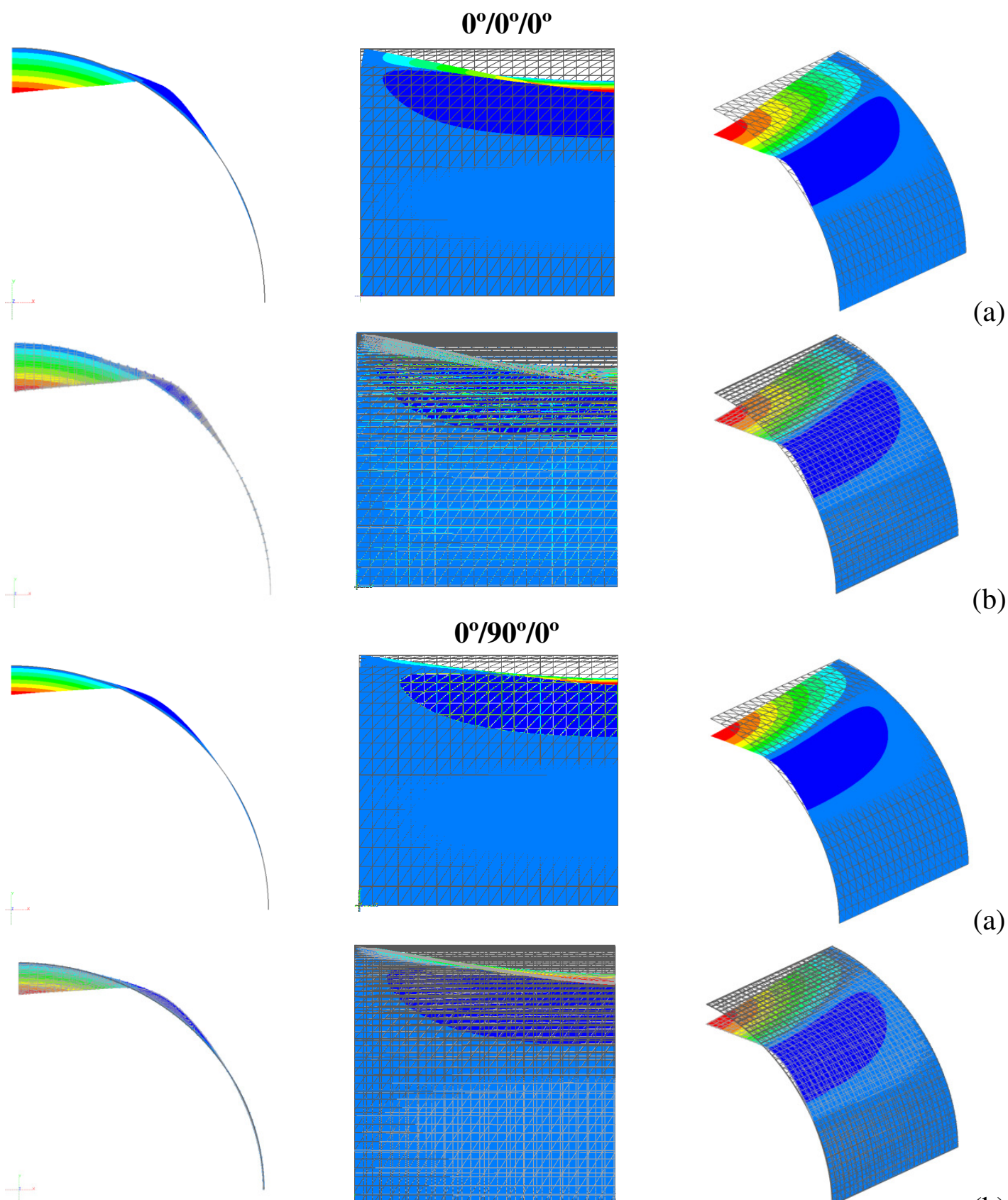

(a)

\section{$0^{\circ} / 90^{\circ} / 0^{\circ}$}
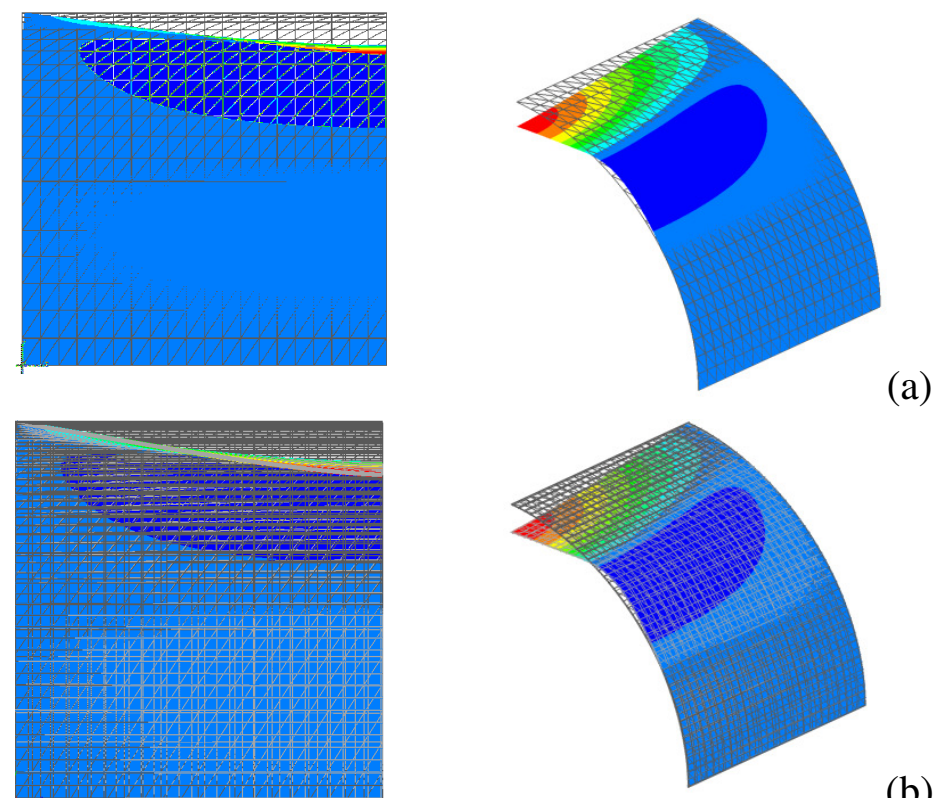

(a)

\section{FIBRAS ALEATÓRIAS}
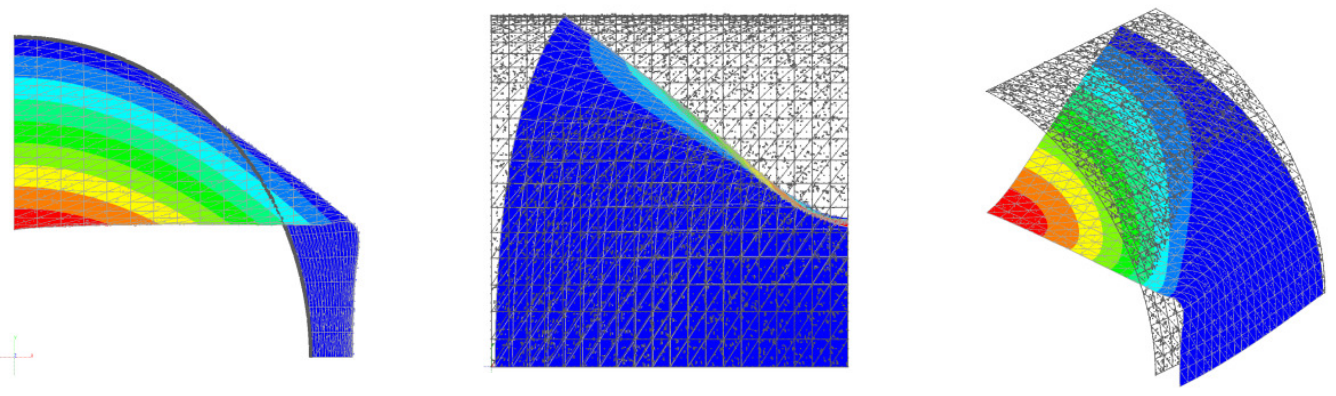

(b)

(c)

Figura 87 - Configuração final de 1/8 do cilindro em grandes deslocamentos para os diferentes arranjos testados: (a) laminada equivalente; (b) laminada com fibras; (c) laminada com fibras aleatórias. 


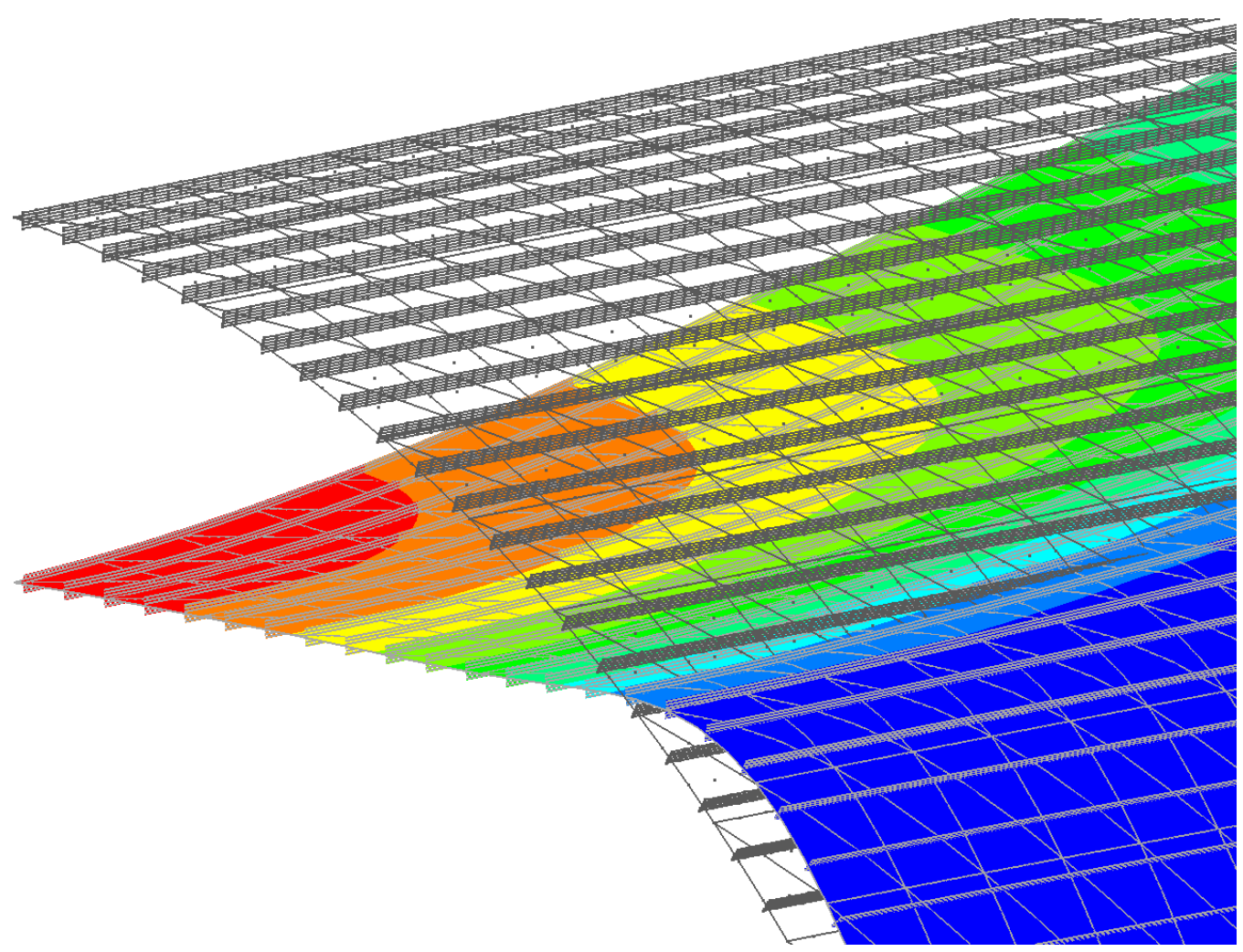

Figura 88 - Vista ampliada da configuração final deformada de 1/8 do cilindro reforçado com fibras: arranjo $0^{\circ} / 0^{\circ} / 0^{\circ}$.

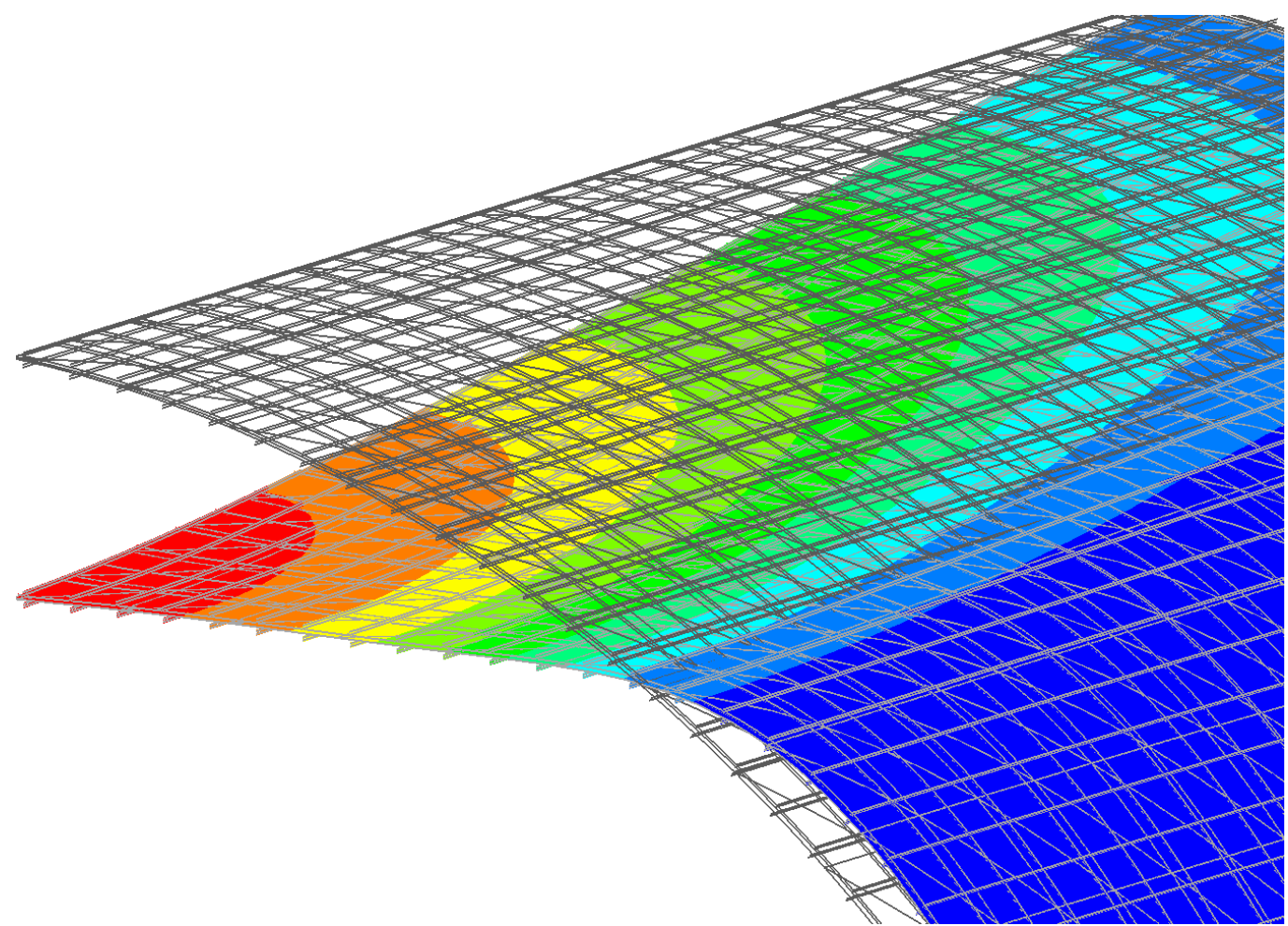

Figura 89 - Vista ampliada da configuração final deformada de 1/8 do cilindro reforçado com fibras: arranjo $0^{\circ} / 90^{\circ} / 0^{\circ}$. 


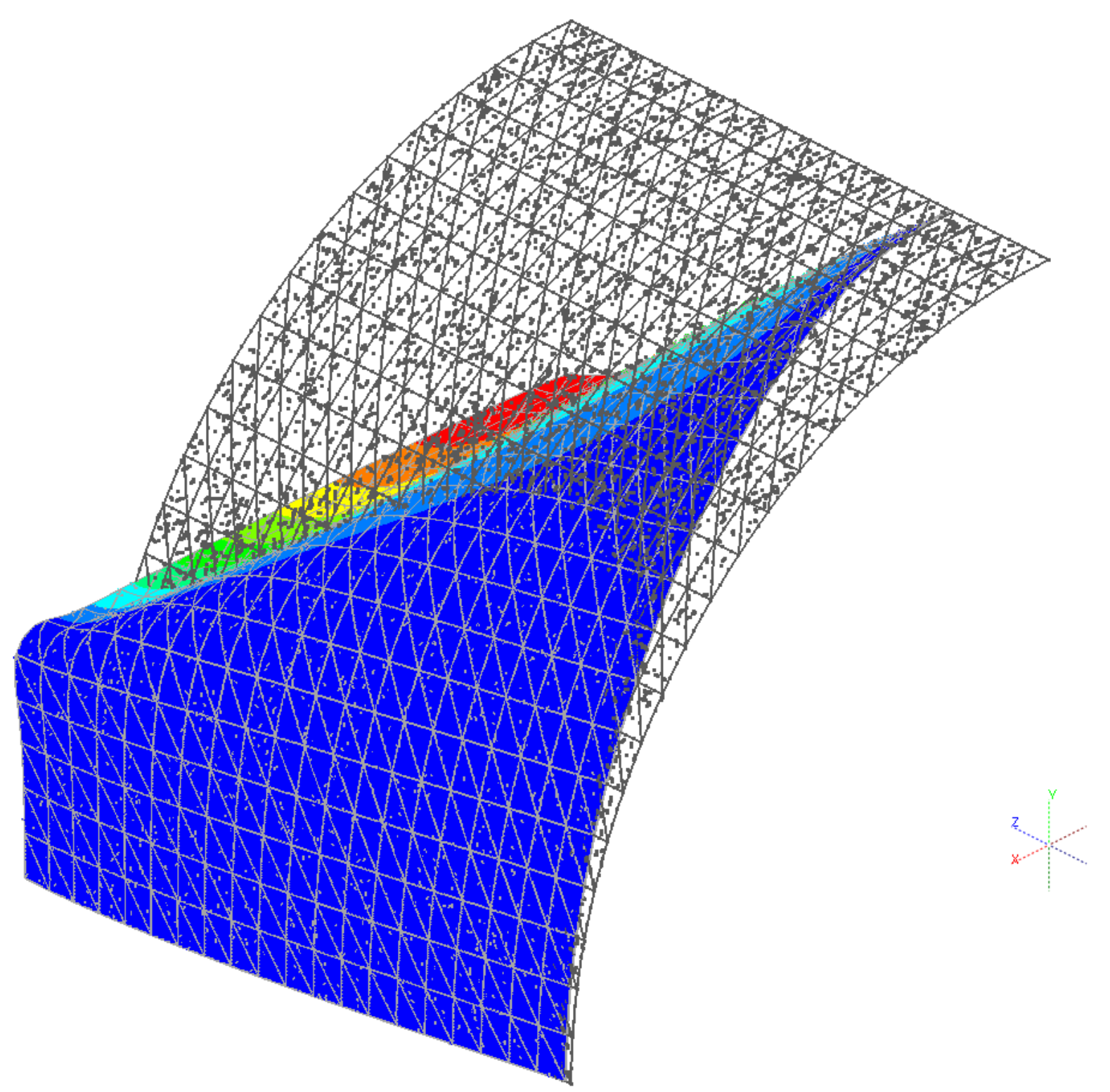

Figura 90 - Vista ampliada da configuração deformada de 1/8 do cilindro reforçado com fibras aleatórias. 

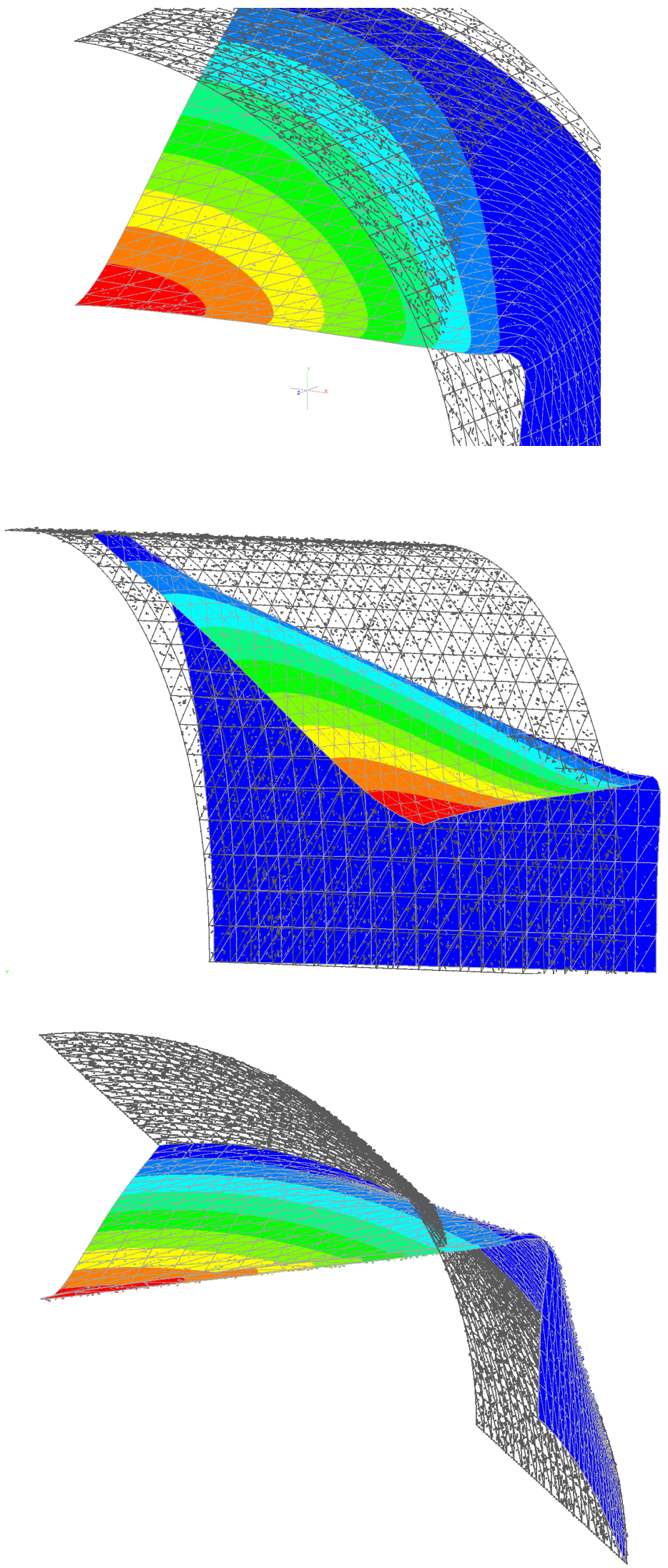

Figura 91 - Outras vistas ampliadas da configuração final deformada de 1/8 do cilindro reforçado com fibras aleatórias. 


\section{CONCLUSÃO}

Uma formulação para a inclusão de fibras longas e curtas, aleatórias ou não, nas diversas lâminas de cascas laminadas anisotrópicas sem o aumento do número de graus de liberdade do problema com a consideração de não linearidade geométrica através do método dos elementos finitos com parâmetros nodais em posições foi proposta e implementada com sucesso neste trabalho.

Elementos de fibras curvas de ordem qualquer, utilizando polinômios de Lagrange e lei constitutiva unidimensional, foram desenvolvidos especialmente para a análise de meios reforçados. Estes elementos foram introduzidos em meios contínuos, chapas, placas e cascas, modelados por elementos finitos triangulares de ordem cúbica.

A estratégia de inclusão, baseada nos conceitos de conjugado energético força-posição associado a relações cinemáticas entre uma posição qualquer do contínuo e os nós de fibra, foi desenvolvida em uma versão generalizada ainda não disponível na literatura, portanto original, e testada com sucesso nos exemplos apresentados ao longo da tese.

Um programa de chapa com elementos finitos triangulares de ordem qualquer foi integralmente implementado para testar a ideia original que em seguida foi estendida para aplicações em cascas laminadas. Foi desenvolvida, também de forma original, uma estratégia para o cálculo diferencial das forças de contato entre o contínuo e as fibras. Alternativamente, foi implementada técnica de cálculo de tensões de contato média, onde as forças de transferência nodais são divididas por comprimentos representativos. As duas estratégias foram comparadas no programa de chapa concluindo-se que, apesar de mais elegante, a técnica diferencial apresenta resultados com maiores oscilações do que o cálculo pela média. $\mathrm{Na}$ generalização do cálculo para elementos de casca optou-se por implementar apenas o cálculo de tensões pela média.

Para possibilitar a geração das fibras de forma livre, sem coincidência dos nós das fibras com os nós das discretizações de chapas e cascas, criou-se algoritmo que identifica a qual elemento finito um ponto qualquer do plano pertence e quais são as coordenadas 
adimensionais deste ponto relativas ao elemento finito detectado. Este algoritmo foi testado e utilizado com sucesso nas aplicações de chapas, placas e cascas. Para cascas, limitou-se a aplicação do algoritmo desenvolvido para geração e inclusão automática de fibras a malhas estruturadas, onde a coincidência da numeração de nós e elementos possibilita a aplicação direta das conectividades determinadas ao mapeamento da casca.

A partir de todas as análises realizadas conclui-se que o algoritmo de geração, a estratégia de inclusão de fibras baseada em conjugados energéticos, o cálculo das tensões de contato e, portanto, todas as propostas desta tese estão corretas e apresentaram os resultados esperados. No que diz respeito aos comportamentos físicos interpretados pelos resultados obtidos dois comentários importantes podem ser feitos.

O primeiro é que a utilização de método numérico que considera fibras diretamente imersas nos domínios pode ser uma estratégia alternativa às técnicas de homogeneização bastante utilizadas atualmente, tendo em vista a grande dificuldade em se determinar propriedades elásticas representativas para meios ortotrópicos ou anisotrópicos constituídos por esse tipo de material, principalmente quando as fibras são espalhadas de forma aleatória no meio de interesse.

O segundo é que os cálculos de tensões de contato entre fibra e matriz em elementos finitos é um problema bastante difícil de ser resolvido. Esta dificuldade se deve ao fato do campo de deformação e, consequentemente de tensão, não ser contínuo entre elementos finitos e no caso da presença de grandes gradientes, como nas tensões de contato tratadas neste problema, a simples média entre valores não é capaz de regularizar os resultados.

Destas conclusões e considerando-se a abrangência dos temas necessários para o desenvolvimento desta tese, tais como, não linearidade geométrica, materiais compósitos, método dos elementos finitos, elementos finitos de cascas laminada, etc., e dadas as potencialidades da formulação proposta e do programa base disponível sugere-se para trabalhos futuros:

1. Desenvolver uma formulação para considerar a não linearidade física na fibra;

2. Desenvolver uma formulação para considerar o deslizamento entre fibra e matriz;

3. Desenvolver uma formulação com dano e enriquecimento de tensão ao longo da espessura do laminado;

4. Desenvolver uma formulação dinâmica não linear geométrica para analisar a influência dos enrijecedores na vibração e amortecimento das estruturas;

5. Considerar outros métodos na resolução do problema de equilíbrio não linear 
geométrico, como comprimento de arco;

6. Paralelizar o código computacional. 


\section{REFERÊNCIAS BIBLIOGRÁFICAS}

AHMAD, S.; IRONS, B.; ZIENKIEWICZ, C. Analysis of thick and thin shell structures by curved finite elements. Int. J. Numer. Meth. Engng., 2, 419-451, 1970.

ANTMAN, S. The eversion of thick spherical shells. Archive for Rational Mechanics and Analysis, 70, 113-123, 1979.

ARCINIEGA, R.; REDDY, J. Consistent third-order shell theory with application to composite circular cylinders. American Institute of Aeronautics and Astronautics (AIAA) Journal, 43(9), 2024-2038, 2005.

ASSAN, A. E. Método dos Elementos finitos: primeiros passos. 2a Edição. Campinas/SP: Editora da Unicamp, 2003.

BABUSKA, I.; MELENK, J. The partition of unity method. Int. J. Numer. Methods Eng., 40, 727-758, 1997.

BALAKRISHNAN, S.; MURRAY, D. Finite element prediction of reinforced concrete. Ph.D. Thesis. University of Alberta, 1986.

BARUT, A.; MADENCI, E.; TESSLER, A.; STARNES JR., J. H. A new stiffened shell element for geometrically nonlinear analysis of composite laminates. Computers and Structures, 77, 11-40, 2000.

BASAR, Y.; ITSKOV, M.; ECKSTEIN, A. Composite laminates: nonlinear interlaminar stress analysis by multi-layer shell elements. Comput. Methods Appl. Mech. Engrg., 185, 367-397, 2000.

BERT, C. A critical evaluation of new plate theories applied to laminated composites. Composite Structures, 2, 329-347, 1984.

BHIMARADDI, A.; CARR, A.; MOSS, P. Finite element analysis of laminated shells of revolution with laminated stiffeners. Computers \& Structures, 33, 295-305, 1989.

BOLANDER JR., J.; SAITO, S. Discrete modeling of short-fibre reinforcement in cementitious composites. Adv. Cem. Based Mater., 6, 76-86, 1997.

BONET, J.; WOOD, R.D.; MAHANET, J.; HEYWOOD, P. Finite element analysis of air supported membrane structures. Comput. Methods Appl. Mech. Eng., 190(5-7), 579-595, 2000. 
CARRAZEDO, R. Estudo e desenvolvimento de código computacional para análise de impacto entre estruturas levando em consideração efeitos térmicos. Tese (Doutorado). Escola de Engenharia de São Carlos, Universidade de São Paulo, São Carlos, 2009.

CHUDOBA, R.; JERÁBEK, J.; PEIFFER, F. Crack-centered enrichment for debonding in two-phase composite applied to textile reinforced concrete. Int. J. Multiscale Comput. Eng., 7(4), 309-328, 2009.

CIARLET, P. Mathematical Elasticity - Volume 1: Three Dimensional Elasticity. NorthHolland, 1988.

CODA, H. B. Análise da vibração livre de meios elásticos bidimensionais pelo método dos elementos de contorno. Dissertação (Mestrado). Escola de Engenharia de São Carlos, Universidade São Paulo, São Carlos, Brasil, 1990.

CODA, H. B. A solid-like FEM for geometrically non-linear 3D frames. Computational Methods in Applied Mechanics and Engineering, 198(47-48), 3712-3722, 2009.

CODA, H. B.; GRECO, M. A simple FEM formulation for large deflection 2D frame analysis based on position description. Computer Methods in Applied Mechanics and Engineering, 193, 3541-3557, 2004.

CODA, H. B.; PACCOLA, R. R. An alternative positional FEM formulation for geometrical non-linear analysis of shells: curved triangular isoparametric elements. Computational Mechanics, 40(1), 185-200, 2007.

CODA, H. B.; PACCOLA, R. R.. A positional FEM formulation for geometrical non-linear analysis of shells. Latin American Journal of Solids and Structures(5), 205-223, 2008.

CODA, H. B.; PACCOLA, R. R. Unsconstrained finite element for geometrical nonlinear dynamic of shells. Mathematical Problems in Engineering, Article ID 575131(2009), 32, 2009.

CODA, H. B.; PACCOLA, R. R. Improved finite element for 3D laminate frame analysis including warping for any cross-section. Applied Mathematical Modelling, 34(4), 1107$1137,2010$.

CODA, H. B.; PACCOLA, R. R. A FEM procedure based on positions and unsconstrained vectors applied to non-linear dynamic of 3D frames. Finite Elements in Analysis and Design, 47(4), 319-333, 2011.

CODA, H. B.; PACCOLA, R. R.; SAMPAIO, M. S. M. Positional description applied to the solution of geometrically non-linear plates and shells. Finite Elements in Analysis and Design, 67, 66-75, 2013.

CRISFIELD, M. Non-linear Finite Element Analysis of Solids and Structures. Volume 1: Essentials. John Wiley \& Sons, 1991. 
DANIEL, I.; ISHAI, O. Engineering mechanics of composite materials. Oxford University Press, Inc., 2006.

DÉSIR, J.-M.; ROMDHANE, M.R.B.; ULM, F.-J.; FAIRBAIRN, E.M.R. Steel-concrete interface: revisiting constitutive and numerical modeling. Comput. Struct., 71, 489-503, 1999.

DUARTE, C.; ODEN, J. An h-p adaptive method usign clouds. Comput. Methods Appl. Mech. Eng., 139, 237-262, 1996.

DUARTE, C.; ODEN, J. H-p clouds-an h-p meshless method. Numer. Methods Partial Differ. E., 12, 673-705, 1996.

DUARTE, C.; BABUSKA, I.; ODEN, J. Generalized finite element methods for threedimensional structural mechanics problems. Comput. Struct., 77, 215-232, 2000.

GILEWSKI, W.; RADWAFLSKA, M. A survey of finite element models for the analysis of moderately thick shells. Finite Elements in Analysis and Design, 9, 1-21, 1991.

GRECO, M.; CODA, H. B. Positional FEM formulation for flexible multi-body dynamic analysis. Journal of Sound and Vibration, 290, 1141-1174, 2006.

GREENE, B.; STROME, D.; WEIKEL, R. Application of the stiffness method to the analysis of shell structures. In Proc. Aviation Conference of ASME. Los Angeles. CA. 1961.

HERAKOVICH, C. Mechanics of fibrous composites. John Wiley \& Sons, Inc., 1998.

HETTICH, T.; HUND, A.; RAMM, E. Modeling of failure in composites by X-FEM and level sets within a multiscale framework. Comput. Methods Appl. Mech. Eng., 197, 414424, 2008.

HOLZAPFEL, G. A. Nonlinear Solid Mechanics - A Continuum Approach for Engineering. Chichester, England: John Wiley \& Sons Ltd., 2000. 455p.

JEYACHANDRABOSE, C.; KIRKHOPE, J. A high precision triangular laminated anisotropic shallow thin shell finite element. Computers \& Structures, 21(4), 701-723, 1985.

JONES, I. Pinched cylinder benchmarks for shear deformable laminated shells. Plast. Rubber Compos., 31(6), 249-261, 2002.

JONES, R. Mechanics of Composite Materials. MC Grawn Hill, New York, 1975.

KANT, T.; KOMMINENI, J. C0 finite element geometrically non-linear analysis of fibre reinforced composite ans sandwich laminates based on a higher-order theory. Computers \& Structures, 45, 511-520, 1992. 
KANT, T.; KOMMINENI, J. Geometrically non-linear transient analysis of laminated composite and sandwich shells with a refined theory and C0 finite elements. Computers \& Structures, 52, 1243-1259, 1994.

LEVISON, M. An accurate, simple theory of the statics and dynamics of elastic plates. Mechanics Research Communications, 7, 343-350, 1980.

LI, L.Y.; APPLEGARTH, I.; BULL, J.W.; BETTES, P.; BOND, T.J.; THOMPSON, P.A. An auto-adaptive finite element analysis software for stiffened plate and shell structures. Advances in Engineering Software, 28, 285-291, 1997a.

LI, L.Y.; BETTESS, P.; BULL, J.W.; BOND, T.J. Adaptive finite element analysis of stiffened shells. Advances in Engineering Software, 28, 501-507, $1997 \mathrm{~b}$.

LI, Z.; LARA, M. P.; BOLANDER, J. Restraining effects of fibers during non-uniform drying of cement composites. Cem. Concr. Res., 36, 1643-1652, 2006.

LIANGXIN, S.; ZHIYU, S. The analysis of laminated composite plates based on the simple higher-order theory. Computers \& Structures, 43, 831-837, 1992.

LIAO, C.-L.; REDDY, J. Analysis of anisotropic, stiffened composite laminates using a continuum-bases shell element. Computers \& Structures, 34, 805-815, 1990.

LIU, Y.; SOH, C.-K. Shear correction for Mindlin type plate and shell elements. International Journal for Numerical Methods in Engineering, 69, 2789-2806, 2006.

LO, K.; CHRISTENSES, R.; WU, E. A high-order theory of plate deformation. Part 2: laminated plates. Journal of Applied Mechanics, 44, 669-676, 1997.

LUENBERG, D. Linear and nonlinear programming. Addison-Wesley Publishing Company, 1989.

MACIEL, D. N. Análise de problemas elásticos não lineares geométricos empregando o método dos elementos finitos posicional. Tese (Doutorado). Escola de Engenharia de São Carlos, Universidade de São Carlos, São Carlos, 2008.

MACKERLE, J. Finite and boundary element analyses of shells: A bibliography (1990-1992). Finite Elements in Analysis and Design, 14, 73-83, 1993.

MACKERLE, J. Finite-and boundary-element linear and nonlinear analyses of shells and shell-like structures. A bibliography (1999-2001). Finite Elements in Analysis and Design(38), 765-782, 2002.

MARQUES, G. Estudo e desenvolvimento de código computacional baseado no MEF para análise dinâmica não linear geométrica de sólidos bidimensionais. Dissertação (Mestrado). Escola de Engenharia de São Carlos, Universidade de São Paulo, São Carlos, 2006. 
MATTHEWS, F.; RAWLINGS, R. Composite materials: Engineering and Science. Chapman \& Hall, 1994.

MELENK, J.; BABUSKA, I. The partition of unity finite element method: basic theory and applications. Comput. Methods Appl. Mech. Eng., 139, 289-314, 2006.

MINSKI, R. L. Aprimoramento de formulação de identificação e solução do impacto bidimensional entre estrutura e anteparo rígido. Dissertação (Mestrado). Escola de Engenharia de São Carlos, Universidade de São Paulo, São Carlos, 2008.

ODEN, J. T. Mechanics of elastic structures. New York: McGraw-Hill Book Company, 1967.

ODEN, J.; DUARTE, C.; ZIENKIEWICZ, O. A new cloud-based hp finite element method. Comput. Methods Appl. Mech. Eng., 153, 117-126, 1998.

OGDEN, R. W. Non-linear elastic deformation. Chichester, Ellis Horwood. Nova Iorque, 1984.

OLIVER, J.; LINERO, D.L.; HUESPE, A.E.; MANZOLI, O.L. Two-dimensional modeling of material failure in reinforced concrete by means of a continuum strong discontinuity approach. Comput. Methods Appl. Mech. Eng., 197, 332-348, 2008.

PASCON, J. P. Modelos constitutivos para materiais hiperelásticos: estudo e implementação computacional. Dissertação (Mestrado). Departamento de Engenharia de Estruturas, Escola de Engenharia de São Carlos, Universidade de São Paulo, São Carlos, 2008.

PASCON, J. P. Sobre modelos constitutivos não lineares para materiais com gradação funcional exibindo grandes deformações: implementação numérica em formulação não linear geométrica. Tese (Doutorado). Departamento de Engenharia de Estruturas, Escola de Engenharia de São Carlos, Universidade de São Paulo, São Carlos, 2012.

PHAN, N.; REDDY, J. Analysis of laminated composite plates using a higher-order shear deformation theory. International Journal for Numerical Methods in Engineering, 21, 2201-2219, 1985.

PRUSTY, B. Linear static analysis of composite hat-stiffened laminated shells using finite elements. Finite Elements in Analysis and Design, 39, 1125-1138, 2003.

PRUSTY, B.; SATSANGI, S. Analysis of stiffened shell for ships and ocean structures by finite elemen method. Ocean Engineering, 28, 621-638, 2001.

QATU, M.; ASADI, E.; WANG, W. Review of recent literature on static analyses of composite shells: 2000-2010. Open Journal of Composite Materials, 2, 61-86, 2012.

QATU, M.; SULLIVAN, R.; WANG, W. Recent research advances on the dynamic analysis of composite shells: 2000-2009. Composite Structures, 93, 14-31, 2010. 
RADTKE, F.; SIMONE, A.; SLUYS, L. A partition of unity finite element method for obtaining elastic properties of continua with embedded thin fibres. International Journal for Numerical Methods in Engineering, 6(84), 708-732, 2010a.

RADTKE, F.; SIMONE, A.; SLUYS, L. A computational model for failure analysis of fibre reinforced concrete with discrete treatment of fibres. Eng. Fract. Mech., 77(4), 597-620, 2010b.

RADTKE, F.; SIMONE, A.; SLUYS, L. A partition of unity finte element method for simulating nonlinear debonding and matriz failure in thin fibre composites. International Journal for Numerical Methods in Engineering, 86(4-5), 453-476, 2011.

RAMESH, S.S.; WANG, C.M.; REDDY, J.N.; ANG. K.K. A higher-order plate element for accurate prediction of interlaminar stresses in laminated composite plates. Composite Structures, 91, 337-357, 2009.

REDDY, J. A simple higher-order theory for laminated composite plates. Journal of Applied Mechanics, 51, 745-752, 1983.

REDDY, J. A refined nonlinear theory of plates with transverse shear deformation. International Journal of Solids and Structures, 20, 881-896, 1984.

REDDY, J.; LIU, C. A higher-order shear deformation theory of laminated elastic shells. International Journal of Engineering Science, 23, 319-330, 1985.

RIGOBELLO, R. Desenvolvimento e aplicação de código computacional para análise de estruturas de aço aporticadas em situação de incêndio. Tese (Doutorado). Escola de Engenharia de São Carlos, Universidade de São Paulo, São Carlos, 2011.

SAMANTA, A.; MUKHOPADHYAY, M. Finite element large deflection static analysis of shallow and deep stiffened shells. Finite Elements in Analysis and Design, 33, 187-208, 1999.

SAMARTIN, A. Numerical methods in nonlinear analysis of shell structures. Journal of the International Association for Shell and Spatial Structures, 34, 81-102, 1993.

SAMPAIO, M. S. M.; PACCOLA, R. R.; CODA, H. B. Fully adherent fiber-matrix FEM formulation for geometrically nonlinear 2D solid analysis. Finite Elements in Analysis and Design, 66, 12-25, 2013.

SANCHES, R. A. K. Sobre o acoplamento fluido-casca utilizando o Método dos Elementos Finitos. Escola de Engenharia de São Carlos, Universidade de São Paulo, São Carlos, 2011.

SANCHEZ, J. A. G. Uma formulação em elementos finitos para a análise dinâmica e estática não linear de risers incluindo o contato com o leito do mar. Tese (Doutorado). Departamento de Engenharia de Estruturas, Escola de Engenharia de São Carlos, Universidade de São Paulo, São Carlos, 2013. 
SANSOUR, C.; KOLLMANN, F. Families of 4-nodes and 9-nodes finite elements for a finite deformation shell theory. Anassessment of hybrid stress, hybrid strain and enhanced strain elements. Computational Mechanics, 24, 435-447, 2000.

SCHLANGEN, E.; VAN MIER, J. Simple lattice model for numerical simulation of fracture of concrete materials and structures. Mater. Struct., 25, 534-542, 1992.

SOKOLNIKOFF, I. Mathematical theory of elasticity. 2a Edição. New York: McGraw Hill, 1956.

TAUCHERT, T. R. Energy principles in structural mechanics. McGraw Hill Book Company, 1974.

VALLIAPPAN, S. Continuum mechanics fundamentals. Rotterdam: Distributed in USA \& Canada by MBS, 1981.

VANALLI, L. O MEC e o MEF aplicados à análise de problemas viscoplásticos em meios anisotrópicos e composto. Tese (Doutorado). Departamento de Engenharia de Estruturas, Escola de Engenharia de São Carlos, Universidade de São Paulo, São Carlos, 2004.

VANALLI, L.; PACCOLA, R. R.; CODA, H. B. A simple way to introduce fibers into FEM models. Commun. Numer. Methods Eng., 24, 585-603, 2008.

VENKATESH, A.; RAO, K. Analysis of laminated shells with laminated stiffeners using rectangular shell finite elements. Computers Methods in Applied Mechanics and Engineering, 38, 255-272, 1983.

VENKATESH, A.; RAO, K. Analysis of laminated shells of revolution with laminated stiffeners using a doubly curved quadrilateral finite element. Computers \& Structures, 20, 669-682, 1985.

VILLAÇA, S. F.; GARCIA, L. Introdução à teoria da elasticidade. 4a Edição. Rio de Janeiro: COPPE/UFRJ, 2000.

VISON, J.; SIERAKOWSKI, R. The behavior of structures composed of composite materials. Martinus Nijhoff Publishers, 1987.

YANG, H.; SAIGAL, S.; LIAW, D. Advances of thin shell finite elements and some applications - version I. Computers \& Structures, 35, 481-504, 1990.

YANG, H.T.Y.; SAIGAL, S.; MASUD, A.; KAPANIA, R.K. A survey of recent shell finite elemens. Int. J. Numer. Meth. Engng, 47, 101-127, 2000.

YANG, Y.; SHIEH, M. Solution method for nonlinear problems with multiple critical points. AIAAJ, 28, 2110-2116, 1990.

YASIN, M.; KAPURIA, S. An efficient layerwise finite element for shallow composite and sandwich shells. Composite Structures, 98, 202-214, 2013. 
ZHANG, Y.; YANG, C. Recent developments in finite element analysis for laminated composite plates. Composite Structures, 88, 147-157, 2009.

ZUDANS, Z. Analyses of asymmetric stiffened shell type structures by the finite element method: 1. Flat rectangular elements. Nuclear Engineering and Design, 8, 367-379, 1968.

ZUDANS, Z. Analysis of asymmetric stiffened shell type structures by the finite element method: III. Constant transverse shear model. Nuclear Engineering and Design, 11, 177194, 1970. 


\section{APÊNDICE A - VETOR DE FORÇAS INTERNAS E MATRIZ HESSIANA DO ELEMENTO DE FIBRA SIMPLES}

Neste anexo apresentam-se as expressões analíticas das componentes do vetor de forças internas e da matriz Hessiana do elemento finito de fibra simples.

\section{A.1. Vetor de forças internas}

Lembra-se do Capítulo 4 que a expressão para encontrar as componentes do vetor de forças internas do elemento de fibra é dada por:

$$
\bar{F}_{i}{ }^{i n t}=\frac{1}{2} \overline{\mathbb{E}} \bar{E} \bar{A} \bar{L}_{0} \frac{\partial \bar{E}}{\partial \bar{Y}_{i}},
$$

ou ainda,

$$
\bar{F}^{i n t}=\left\{\begin{array}{l}
\bar{F}_{1}^{i n t} \\
\bar{F}_{2}^{i n t} \\
\bar{F}_{3}^{\text {int }} \\
\bar{F}_{4}^{\text {int }}
\end{array}\right\},
$$

cujas componentes são dadas após efetuada a derivada da deformação de Green em relação aos parâmetros nodais por:

$$
\begin{gathered}
\bar{F}_{1}^{i n t}=\frac{\overline{\mathbb{E}} \bar{A}}{\bar{L}_{0}} \bar{E}\left(\bar{Y}_{1}-\bar{Y}_{3}\right), \bar{F}_{2}^{i n t}=\frac{\overline{\mathbb{E}} \bar{A}}{\bar{L}_{0}} \bar{E}\left(\bar{Y}_{2}-\bar{Y}_{4}\right), \\
\bar{F}_{3}^{i n t}=\frac{\overline{\mathbb{E}} \bar{A}}{\overline{L_{0}}} \bar{E}\left(\bar{Y}_{3}-\bar{Y}_{1}\right), F_{4}^{i n t}=\frac{\overline{\mathbb{E}} \bar{A}}{\bar{L}_{0}} \bar{E}\left(\bar{Y}_{4}-\bar{Y}_{2}\right) .
\end{gathered}
$$

\section{A.2. Matriz Hessiana}

As componentes da matriz Hessiana do elemento de fibra simples podem ser obtidas a partir da expressão: 


$$
\bar{H}_{i j}=\frac{\partial}{\partial \bar{Y}_{j}}\left(\frac{1}{2} \overline{\mathbb{E}} \bar{E} \bar{A} \bar{L}_{0} \frac{\partial \bar{E}}{\partial \bar{Y}_{i}}\right),
$$

que podem ser organizadas matricialmente como:

$$
\bar{H}=\left[\begin{array}{llll}
\bar{H}_{11} & \bar{H}_{12} & \bar{H}_{13} & \bar{H}_{14} \\
\bar{H}_{21} & \bar{H}_{22} & \bar{H}_{23} & \bar{H}_{24} \\
\bar{H}_{31} & \bar{H}_{32} & \bar{H}_{33} & \bar{H}_{34} \\
\bar{H}_{41} & \bar{H}_{42} & \bar{H}_{43} & \bar{H}_{44}
\end{array}\right] .
$$

As expressões analíticas destas componentes são dadas por:

$$
\begin{aligned}
& \bar{H}_{11}=\frac{\overline{\mathbb{E}} \bar{A}}{\bar{L}_{0}}\left[\frac{\left(\bar{Y}_{1}-\bar{Y}_{3}\right)^{2}}{\bar{L}_{0}^{2}}+\bar{E}\right], \bar{H}_{12}=\frac{\overline{\mathbb{E}} \bar{A}}{\bar{L}_{0}}\left[\frac{\left(\bar{Y}_{2}-\bar{Y}_{4}\right)\left(\bar{Y}_{1}-\bar{Y}_{3}\right)}{\bar{L}_{0}^{2}}\right], \\
& \bar{H}_{13}=-\frac{\overline{\mathbb{E}} \bar{A}}{\bar{L}_{0}}\left[\frac{\left(\bar{Y}_{1}-\bar{Y}_{3}\right)^{2}}{\bar{L}_{0}^{2}}+\bar{E}\right], \bar{H}_{14}=\frac{\overline{\mathbb{E}} \bar{A}}{\bar{L}_{0}}\left[\frac{\left(\bar{Y}_{4}-\bar{Y}_{2}\right)\left(\bar{Y}_{1}-\bar{Y}_{3}\right)}{\bar{L}_{0}^{2}}\right] \text {, } \\
& \bar{H}_{21}=\frac{\overline{\mathbb{E}} \bar{A}}{\bar{L}_{0}}\left[\frac{\left(\bar{Y}_{1}-\bar{Y}_{3}\right)\left(\bar{Y}_{2}-\bar{Y}_{4}\right)}{\bar{L}_{0}^{2}}\right], \bar{H}_{22}=\frac{\overline{\mathbb{E}} \bar{A}}{\bar{L}_{0}}\left[\frac{\left(\bar{Y}_{2}-\bar{Y}_{4}\right)^{2}}{\bar{L}_{0}^{2}}+\bar{E}\right] \text {, } \\
& \bar{H}_{23}=\frac{\overline{\mathbb{E}} \bar{A}}{\bar{L}_{0}}\left[\frac{\left(\bar{Y}_{3}-\bar{Y}_{1}\right)\left(\bar{Y}_{2}-\bar{Y}_{4}\right)}{\bar{L}_{0}^{2}}\right], \bar{H}_{24}=-\frac{\overline{\mathbb{E}} \bar{A}}{\bar{L}_{0}}\left[\frac{\left(\bar{Y}_{2}-\bar{Y}_{4}\right)^{2}}{\bar{L}_{0}^{2}}+\bar{E}\right] \text {, } \\
& \bar{H}_{31}=-\frac{\overline{\mathbb{E}} \bar{A}}{\bar{L}_{0}}\left[\frac{\left(\bar{Y}_{3}-\bar{Y}_{1}\right)^{2}}{\bar{L}_{0}^{2}}+\bar{E}\right], \bar{H}_{32}=\frac{\overline{\mathbb{E}} \bar{A}}{\bar{L}_{0}}\left[\frac{\left(\bar{Y}_{2}-\bar{Y}_{4}\right)\left(\bar{Y}_{3}-\bar{Y}_{1}\right)}{\bar{L}_{0}^{2}}\right] \text {, } \\
& \bar{H}_{33}=\frac{\overline{\mathbb{E}} \bar{A}}{\bar{L}_{0}}\left[\frac{\left(\bar{Y}_{3}-\bar{Y}_{1}\right)^{2}}{\bar{L}_{0}^{2}}+\bar{E}\right], \bar{H}_{34}=\frac{\overline{\mathbb{E}} \bar{A}}{\bar{L}_{0}}\left[\frac{\left(\bar{Y}_{4}-\bar{Y}_{2}\right)\left(\bar{Y}_{3}-\bar{Y}_{1}\right)}{\bar{L}_{0}^{2}}\right] \text {, } \\
& \bar{H}_{41}=\frac{\overline{\mathbb{E}} \bar{A}}{\bar{L}_{0}}\left[\frac{\left(\bar{Y}_{1}-\bar{Y}_{3}\right)\left(\bar{Y}_{4}-\bar{Y}_{2}\right)}{\bar{L}_{0}^{2}}\right], \bar{H}_{42}=-\frac{\overline{\mathbb{E}} \bar{A}}{\bar{L}_{0}}\left[\frac{\left(\bar{Y}_{4}-\bar{Y}_{2}\right)^{2}}{\bar{L}_{0}^{2}}+\bar{E}\right] \text {, } \\
& \bar{H}_{43}=\frac{\overline{\mathbb{E}} \bar{A}}{\bar{L}_{0}}\left[\frac{\left(\bar{Y}_{3}-\bar{Y}_{1}\right)\left(\bar{Y}_{4}-\bar{Y}_{2}\right)}{\bar{L}_{0}^{2}}\right], \bar{H}_{44}=\frac{\overline{\mathbb{E}} \bar{A}}{\bar{L}_{0}}\left[\frac{\left(\bar{Y}_{4}-\bar{Y}_{2}\right)^{2}}{\bar{L}_{0}^{2}}+\bar{E}\right] \text {. }
\end{aligned}
$$




\section{APÊNDICE B - RESULTADOS PARA A ANÁLISE DE CONFORMIDAdE DO MAPEAMENTO FIBRA-MATRIZ}

Neste apêndice apresentam-se os resultados obtidos para o Exemplo 2 do Capítulo 4 para o elemento bidimensional com aproximações, linear, quadrática, e do quarto grau.

A Figura 92 e a Tabela 17 mostram os resultados obtidos para o caso de matriz linear e fibra variando de linear ao quarto grau.
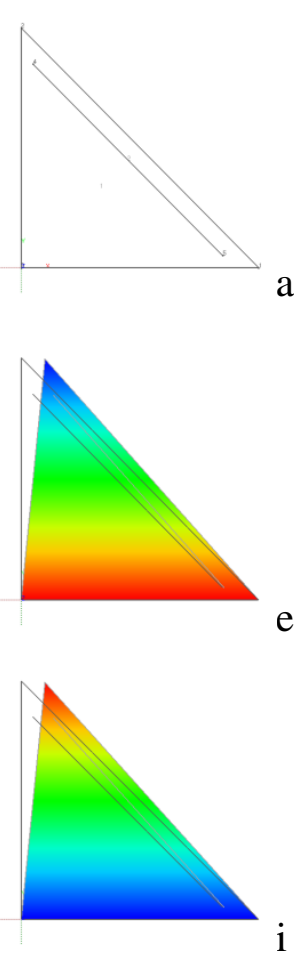
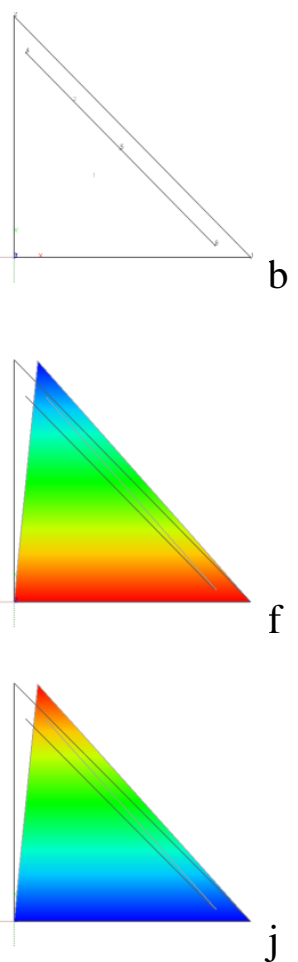
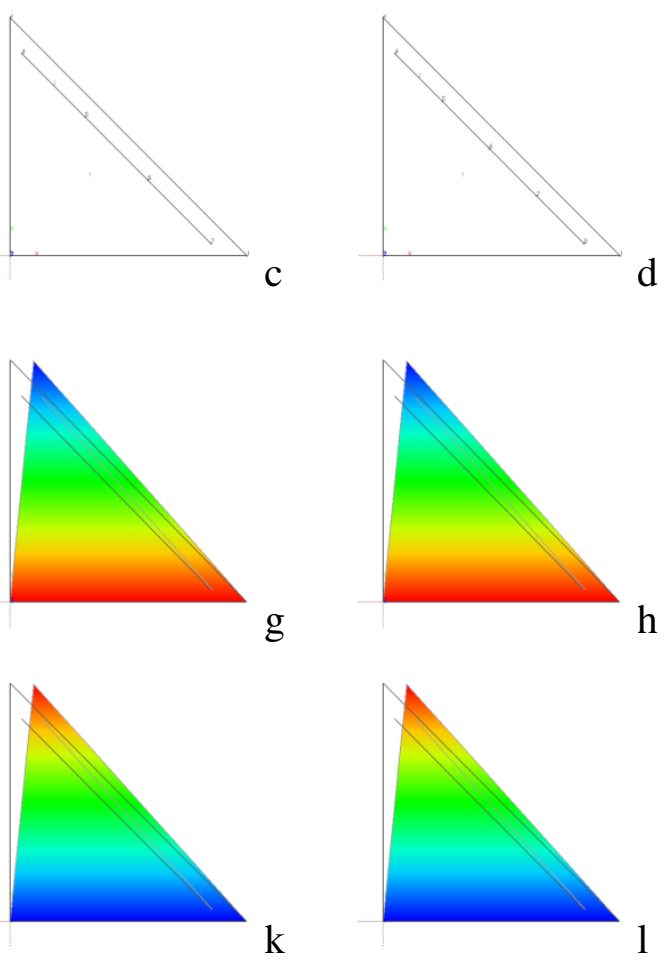

Figura 92 - Matriz com aproximação linear e fibras com aproximação variando de linear ao quarto grau.

Tabela 17 - Matriz com aproximação linear e fibras com aproximação variando de linear ao quarto grau.

\begin{tabular}{|c|c|c|c|c|c|c|c|c|}
\hline \multirow{2}{*}{$\begin{array}{l}\text { GRAUS DE } \\
\text { LIBERDADE }\end{array}$} & \multicolumn{8}{|c|}{ POSIÇŌES } \\
\hline & SÓLIDO: $1^{\circ}$ & FIBRA: $1^{\circ}$ & SÓLIDO: $1^{\circ}$ & FIBRA: $2^{\circ}$ & SÓLIDO: $1^{\circ}$ & FIBRA: $3^{\circ}$ & SÓLIDO: $1^{\circ}$ & FIBRA: $4^{\circ}$ \\
\hline 1 & 27,0000000000000 & 27,0000000000000 & 27,0000000000000 & 27,0000000000000 & 27,0000000000000 & 27,0000000000000 & 27,0000000000000 & 27,0000000000000 \\
\hline 2 & 169,1478643081250 & $\begin{array}{l}169,1478643081250 \\
\end{array}$ & 169,1478643081250 & 169,1478643081250 & 169,1478643081250 & 169,1478643081250 & 169,1478643081250 & 169,1478643081250 \\
\hline 3 & 40,0909090909091 & 40,0909090909091 & 40,0909090909091 & 40,0909090909091 & 40,0909090909091 & 40,0909090909091 & 40,0909090909091 & 40,0909090909091 \\
\hline 4 & 154,6753197683930 & \begin{tabular}{|l|l|}
154,6753197683930 \\
\end{tabular} & 154,6753197683930 & 154,6753197683930 & 154,6753197683930 & 154,6753197683930 & 154,6753197683930 & 154,6753197683930 \\
\hline 5 & 53,1818181818182 & 53,1818181818182 & 53,1818181818182 & 53,1818181818182 & 53,1818181818182 & 53,1818181818182 & 53,1818181818181 & 53,1818181818181 \\
\hline 6 & 140,2027752286600 & 140,2027752286600 & 140,2027752286600 & 140,2027752286600 & 140,2027752286600 & 140,2027752286600 & 140,2027752286600 & 140,2027752286600 \\
\hline 7 & 66,2727272727273 & 66,2727272727273 & 66,2727272727273 & 66,2727272727273 & 66,2727272727273 & 66,2727272727273 & 66,2727272727273 & 66,2727272727273 \\
\hline 8 & 125,7302306889270 & 125,7302306889270 & 125,7302306889270 & 125,7302306889270 & 125,7302306889270 & 125,7302306889270 & 125,7302306889270 & 125,7302306889270 \\
\hline 9 & 79,3636363636364 & 79,3636363636364 & 79,3636363636364 & 79,3636363636364 & 79,3636363636364 & 79,3636363636364 & 79,3636363636364 & 79,3636363636364 \\
\hline 10 & 111,2576861491950 & 111,2576861491950 & 111,2576861491950 & 111,2576861491950 & 111,2576861491950 & 111,2576861491950 & 111,2576861491950 & 111,2576861491950 \\
\hline 11 & 92,4545454545455 & $\begin{array}{l}92,4545454545455 \\
\end{array}$ & 92,4545454545455 & 92,4545454545455 & 92,4545454545455 & 92,4545454545455 & 92,4545454545455 & 92,4545454545455 \\
\hline 12 & 96,7851416094621 & \begin{tabular}{|c|}
96,7851416094621 \\
\end{tabular} & 96,7851416094621 & 96,7851416094621 & 96,7851416094621 & 96,7851416094621 & 96,7851416094621 & 96,7851416094621 \\
\hline 13 & 105,5454545454550 & 105,5454545454550 & 105,5454545454550 & 105,5454545454550 & 105,5454545454550 & 105,5454545454550 & 105,5454545454550 & 105,5454545454550 \\
\hline 14 & 82,3125970697295 & 82,3125970697295 & 82,3125970697295 & 82,3125970697295 & 82,3125970697295 & 82,3125970697295 & 82,3125970697295 & 82,3125970697295 \\
\hline 15 & 118,6363636363640 & 118,6363636363640 & 118,6363636363640 & 118,6363636363640 & 118,6363636363640 & 118,6363636363640 & 118,6363636363640 & 118,6363636363640 \\
\hline 16 & 67,8400525299968 & 67,8400525299968 & 67,8400525299968 & 67,8400525299968 & 67,8400525299968 & 67,8400525299968 & 67,8400525299968 & 67,8400525299968 \\
\hline 17 & 131,7272727272730 & 131,7272727272730 & 131,7272727272730 & 131,7272727272730 & $\begin{array}{l}131,7272727272730 \\
\end{array}$ & 131,7272727272730 & 131,7272727272730 & 131,7272727272730 \\
\hline 18 & 53,3675079902642 & $\begin{array}{l}53,3675079902642 \\
\end{array}$ & 53,3675079902642 & 53,3675079902642 & $\begin{array}{r}53,3675079902642 \\
\end{array}$ & 53,3675079902642 & 53,3675079902642 & 53,3675079902642 \\
\hline 19 & 144,8181818181820 & 144,8181818181820 & 144,8181818181820 & 144,8181818181820 & 144,8181818181820 & 144,8181818181820 & 144,8181818181820 & 144,8181818181820 \\
\hline 20 & $\begin{array}{l}38,8949634505315 \\
\end{array}$ & $\begin{array}{l}38,8949634505315 \\
\end{array}$ & 38,8949634505315 & $\begin{array}{l}38,8949634505315 \\
\end{array}$ & 38,8949634505315 & $\begin{array}{l}38,8949634505315 \\
\end{array}$ & \begin{tabular}{|l}
38,8949634505315 \\
\end{tabular} & $\begin{array}{l}38,8949634505315 \\
\end{array}$ \\
\hline 21 & 157,9090909090910 & \begin{tabular}{|l|}
157,9090909090910 \\
\end{tabular} & $\begin{array}{l}157,9090909090910 \\
\end{array}$ & 157,9090909090910 & 157,9090909090910 & 157,9090909090910 & 157,9090909090910 & 157,9090909090910 \\
\hline 2 & 24,42 & & 24,4 & 24,42 & 77989 & 24,42 & 24,4 & 24,422418910 \\
\hline 23 & 171,0000000000000 & 171,0000000000000 & 171,0000000000000 & 171,0000000000000 & 171,0000000000000 & 171,0000000000000 & 171,0000000000000 & 171,0000000000000 \\
\hline 24 & 9,9498743710662 & $\begin{array}{l}9,9498743710662 \\
\end{array}$ & 9,9498743710662 & 9,9498743710662 & 9,9498743710662 & 9,9498743710662 & 9,9498743710662 & 9,9498743710662 \\
\hline
\end{tabular}


A Figura 93 e a Tabela 18 mostram os resultados obtidos para o caso de matriz quadrática e fibra variando de linear ao quarto grau.
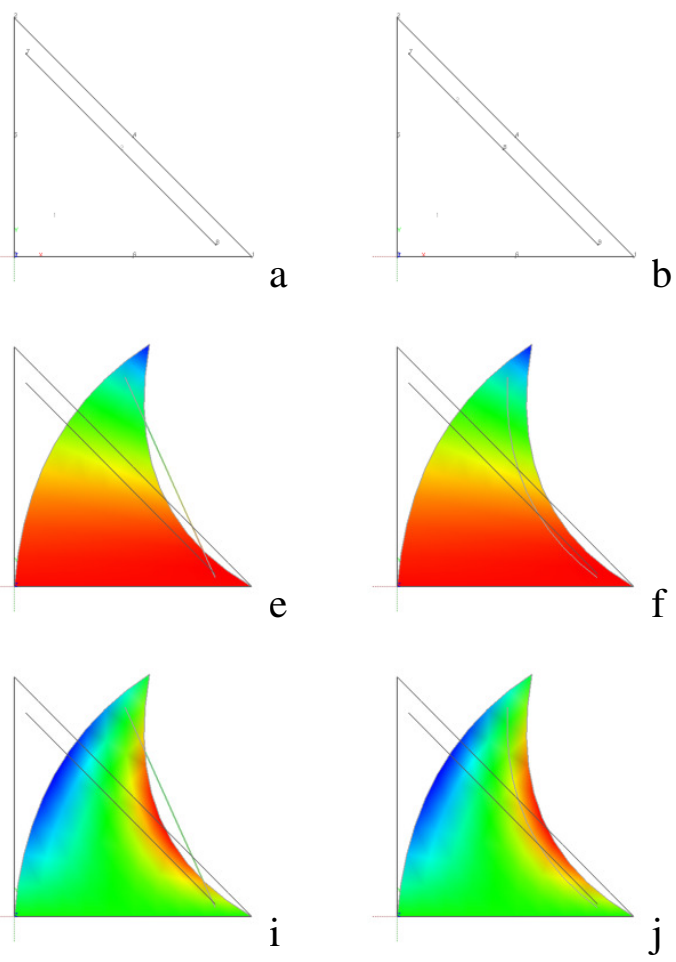
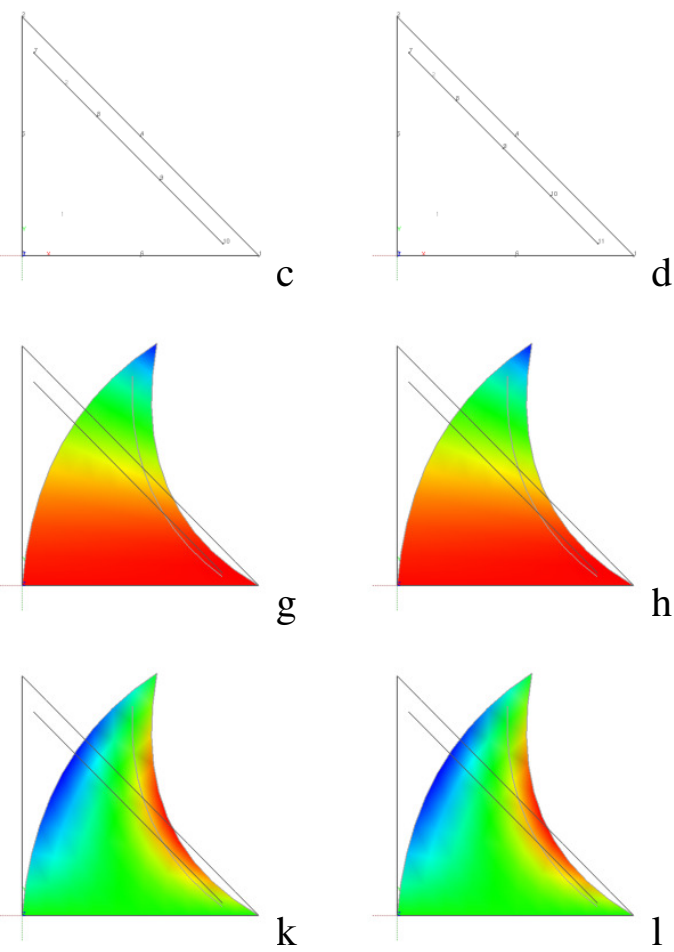

Figura 93 - Matriz com aproximação quadrática e fibras com aproximação variando de linear ao quarto grau.

Tabela 18 - Matriz com aproximação quadrática e fibras com aproximação variando de linear ao quarto grau.

\begin{tabular}{|c|c|c|c|c|c|c|c|c|}
\hline \multirow{2}{*}{$\begin{array}{c}\text { GRAUS DE } \\
\text { LIBERDADE }\end{array}$} & \multicolumn{8}{|c|}{ POSIÇŌES } \\
\hline & SÓLIDO: $2^{\circ}$ & FIBRA: $1^{\circ}$ & SÓLIDO: $2^{\circ}$ & FIBRA: $2^{\circ}$ & SÓLIDO: $2^{\circ}$ & FIBRA: $3^{\circ}$ & SÓLIDO: $2^{\circ}$ & FIBRA: $4^{\circ}$ \\
\hline 4 & 93,36706952715830 & 93,36706952715830 & 93,36706952715830 & 93,36706952715830 & 93,36706952715830 & 93,36706952715830 & 93,36706952715830 & 93,36706952715830 \\
\hline 2 & 174,82808657928200 & 174,82808657928200 & 174,82808657928200 & 174,82808657928200 & 174,82808657928200 & 174,82808657928200 & 174,82808657928200 & 174,82808657928200 \\
\hline 3 & 93,06750392293870 & 100,28341863941400 & 93,06750392293870 & \begin{tabular}{r|}
93,06750392293870 \\
\end{tabular} & 93,06750392293870 & 93,06750392293870 & 93,06750392293870 & 93,06750392293870 \\
\hline 4 & 155,59013912756300 & 159,59425102400300 & 155,59013912756300 & 155,59013912756300 & 155,59013912756300 & 155,59013912756300 & 155,59013912756300 & 155,59013912756300 \\
\hline 5 & 94,21112126201420 & 107,19976775166900 & $\begin{array}{l}94,21112126201420 \\
\end{array}$ & 94,21112126201420 & 94,21112126201420 & 94,21112126201420 & $\begin{array}{l}94,21112126201410 \\
\end{array}$ & 94,21112126201410 \\
\hline 6 & 137,15301405513100 & 144,36041546872400 & 137,15301405513100 & 137,15301405513100 & 137,15301405513100 & 137,15301405513100 & $\begin{array}{l}137,15301405513100 \\
\end{array}$ & 137,15301405513100 \\
\hline 7 & 96,79792154438470 & 114,11611686392500 & 96,79792154438470 & 96,79792154438470 & 96,79792154438470 & 96,79792154438470 & 96,79792154438470 & 96,79792154438470 \\
\hline 8 & 119,51671136198800 & 129,12657991344600 & 119,51671136198800 & 119,51671136198800 & 119,51671136198800 & 119,51671136198800 & 119,51671136198800 & 119,51671136198800 \\
\hline 9 & 100,82790477005000 & 121,03246597618000 & 100,82790477005000 & 100,82790477005000 & 100,82790477005000 & 100,82790477005000 & 100,82790477005000 & 100,82790477005000 \\
\hline 10 & 102,68123104813300 & 113,89274435816700 & 102,68123104813300 & 102,68123104813300 & 102,68123104813300 & 102,68123104813300 & 102,68123104813300 & 102,68123104813300 \\
\hline 11 & 106,30107093901100 & 127,94881508843600 & 106,30107093901100 & 106,30107093901100 & 106,30107093901100 & 106,30107093901100 & 106,30107093901100 & 106,30107093901100 \\
\hline 12 & 86,64657311356610 & 98,65890880288820 & 86,64657311356610 & 86,64657311356610 & 86,64657311356610 & 86,64657311356610 & 86,64657311356610 & 86,64657311356610 \\
\hline 13 & 113,21742005126600 & 134,86516420069100 & 113,21742005126600 & 113,21742005126600 & 113,21742005126600 & 113,21742005126600 & 113,21742005126600 & 113,21742005126600 \\
\hline 14 & 71,41273755828730 & $\begin{array}{l}83,42507324760950 \\
\end{array}$ & 71,41273755828740 & $\begin{array}{l}71,41273755828730 \\
\end{array}$ & 71,41273755828730 & 71,41273755828740 & 71,41273755828730 & 71,41273755828730 \\
\hline 15 & 121,57695210681700 & 141,78151331294700 & 121,57695210681700 & 121,57695210681700 & 121,57695210681700 & 121,57695210681700 & 121,57695210681700 & 121,57695210681700 \\
\hline 16 & 56,97972438229670 & 68,19123769233070 & 56,97972438229670 & 56,97972438229670 & 56,97972438229670 & 56,97972438229670 & $\begin{array}{l}56,97972438229670 \\
\end{array}$ & 56,97972438229670 \\
\hline 17 & 131,37966710566200 & 148,69786242520300 & 131,37966710566200 & 131,37966710566200 & $\begin{array}{l}131,37966710566200 \\
\end{array}$ & 131,37966710566200 & $\begin{array}{l}131,37966710566200 \\
\end{array}$ & 131,37966710566200 \\
\hline 18 & 433,34753358559420 & 52,95740213705190 & 43,34753358559420 & 43,34753358559420 & 43,34753358559420 & 43,34753358559420 & 43,34753358559420 & 43,34753358559420 \\
\hline 19 & 142,62556504780300 & 155,61421153745800 & 142,62556504780300 & 142,62556504780300 & 142,62556504780300 & 142,62556504780300 & 142,62556504780300 & 142,62556504780300 \\
\hline 20 & 30,51616516817990 & 37,72356658177320 & 30,51616516817990 & 30,51616516817990 & 30,51616516817990 & 30,51616516817990 & 30,51616516817990 & 30,51616516817990 \\
\hline 21 & 155,31464593323900 & 162,53056064971400 & 155,31464593323900 & 155,31464593323900 & 155,31464593323900 & 155,31464593323900 & $\begin{array}{l}155,31464593323900 \\
\end{array}$ & 155,31464593323900 \\
\hline 22 & 18,4856 & 22,48973102649440 & 18,48561913005370 & 18,48561913005370 & 18,48561913005370 & 18,48561913005370 & 18,48561913005370 & 18,48561913005370 \\
\hline 23 & 169,44690976196900 & 169,44690976196900 & 169,44690976196900 & 169,44690976196900 & $\begin{array}{l}169,44690976196900 \\
\end{array}$ & 169,44690976196900 & \begin{tabular}{ll|l}
169,44690976196900 \\
\end{tabular} & 169,44690976196900 \\
\hline 24 & 7,25589547121566 & 7,25589547121566 & $\begin{array}{l}7,25589547121566 \\
\end{array}$ & 7,25589547121566 & 7,25589547121566 & 7,25589547121566 & 7,25589547121566 & 7,25589547121566 \\
\hline
\end{tabular}

A Figura 94 e a Tabela 19 mostram os resultados obtidos para o caso de matriz do quarto grau e fibra variando de linear ao quarto grau. 

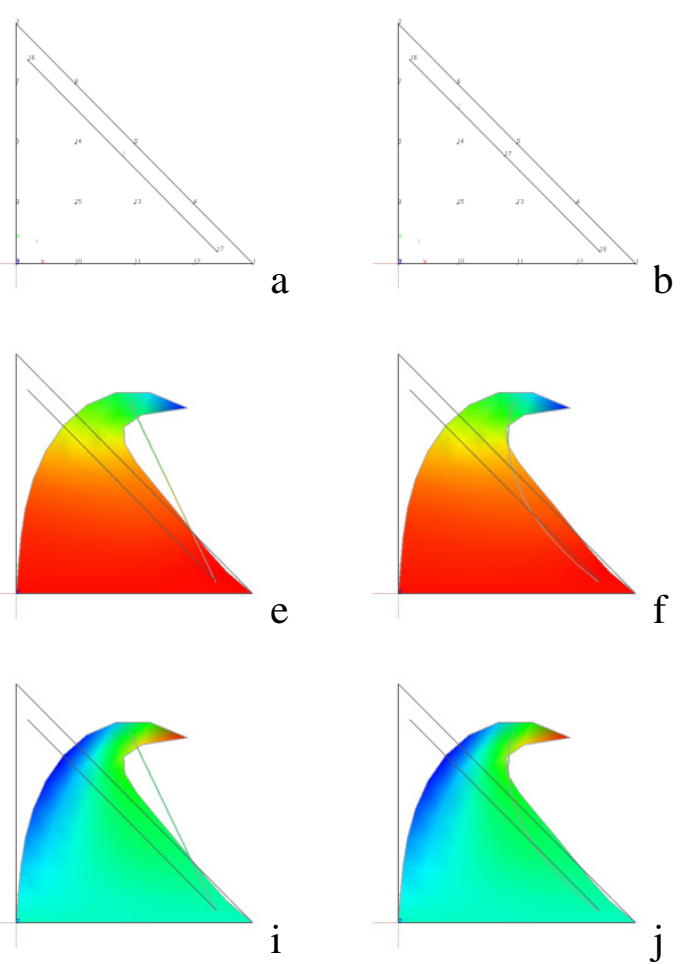
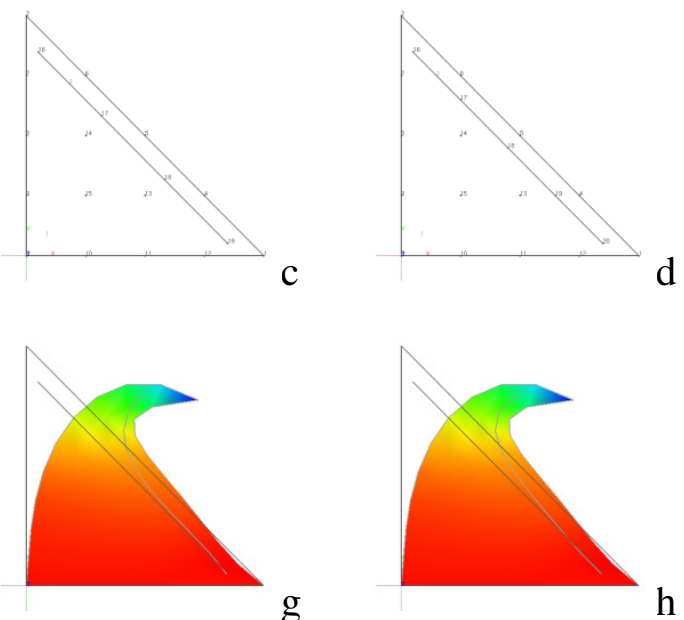

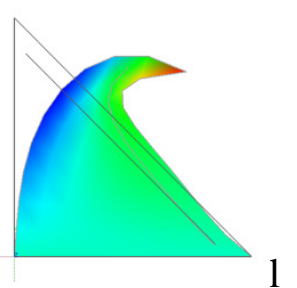

Figura 94 - Matriz com aproximação do quarto grau e fibras com aproximação variando de linear ao quarto grau.

Tabela 19 - Matriz com aproximação do quarto grau e fibras com aproximação variando de linear ao quarto grau.

\begin{tabular}{|c|c|c|c|c|c|c|c|c|}
\hline \multirow{2}{*}{$\begin{array}{l}\text { GRAUS DE } \\
\text { LIBERDADE }\end{array}$} & \multicolumn{8}{|c|}{ POSIÇŌES } \\
\hline & SÓLIDO: $4^{\circ}$ & FIBRA: $1^{\circ}$ & SÓLIDO: $4^{\circ}$ & FIBRA: $2^{\circ}$ & SÓLIDO: $4^{\circ}$ & FIBRA: $3^{\circ}$ & SÓLIDO: $4^{\circ}$ & FIBRA: $4^{\circ}$ \\
\hline 1 & 96,4180217928292 & 96,4180217928292 & 96,4180217928292 & 96,4180217928292 & 96,4180217928292 & 96,4180217928292 & 96,4180217928295 & 96,4180217928292 \\
\hline 2 & 159,1391051929030 & 159,1391051929030 & 159,1391051929030 & 159,1391051929030 & 159,1391051929030 & 159,1391051929030 & 159,1391051929030 & 159,1391051929030 \\
\hline 3 & 83,0597149904298 & 103,0200398717300 & 83,0597149904301 & 93,5595782026024 & $\begin{array}{r}83,0597149904294 \\
\end{array}$ & 86,3716184214077 & 83,0597149904298 & 83,0597149904303 \\
\hline 4 & 145,6738987006200 & 145,5195230587460 & 145,6738987006210 & 145,0219168291020 & 145,6738987006190 & 145,3304973160970 & 145,6738987006200 & 145,6738987006200 \\
\hline 5 & 79,1288455842682 & 109,6220579506300 & 79,1288455842678 & 92,5932269462010 & 79,1288455842682 & 82,2664383083520 & 79,1288455842676 & 79,1288455842679 \\
\hline 6 & 131,7889265780490 & 131,8999409245890 & 131,7889265780490 & 131,0042497112290 & 131,7889265780490 & 131,4635989506060 & 131,7889265780490 & 131,7889265780490 \\
\hline 7 & 81,8723449504581 & 116,2240760295300 & 81,8723449504581 & 93,5189680236251 & 81,8723449504584 & 83,2319684642281 & 81,8723449504584 & 81,8723449504583 \\
\hline 8 & 117,7114172807260 & 118,2803587904320 & 117,7114172807260 & 117,0861038392850 & 117,7114172807270 & 117,5704419755020 & 117,7114172807270 & 117,7114172807270 \\
\hline 9 & 89,0077833737295 & 122,8260941084310 & 89,0077833737302 & $\begin{aligned} 96,3368014348746 \\
\end{aligned}$ & $\begin{array}{r}89,0077833737296 \\
\end{array}$ & $\begin{array}{l}88,3976958996024 \\
\end{array}$ & 89,0077833737293 & 89,0077833737298 \\
\hline 10 & 103,6198001200750 & 104,6607766562750 & 103,6198001200760 & 103,2674792132700 & 103,6198001200750 & 103,6830582698560 & 103,6198001200750 & 103,6198001200760 \\
\hline 11 & 98,7233700474236 & 129,4281121873310 & 98,7233700474237 & 101,0467271799490 & 98,7233700474236 & 96,8931076250411 & 98,7233700474237 & 98,7233700474234 \\
\hline 12 & 89,6437052633986 & 91,0411945221184 & 89,6437052633989 & 89,5483758331837 & 89,6437052633986 & 89,8334797127405 & 89,6437052633987 & 89,6437052633987 \\
\hline 13 & 109,6779530734920 & 136,0301302662310 & 109,6779530734920 & 107,6487452588500 & 109,6779530734920 & 107,8476906511100 & 109,6779530734920 & 109,6779530734930 \\
\hline 14 & 75,8639637338843 & 77,4216123879614 & 75,8639637338847 & 75,9287936990267 & 75,8639637338843 & 76,0537381832262 & 75,8639637338842 & 75,8639637338844 \\
\hline 15 & 121,0010194625040 & 142,6321483451320 & 121,0010194625040 & 116,1428556715760 & 121,0010194625040 & 120,3909319883760 & 121,0010194625040 & 121,0010194625050 \\
\hline 16 & 4106042 & 63,8020302538043 & 62,3126074106043 & 62,4087328107986 & 62,3126074106039 & 62,3758655603848 & 62,3126074106042 & 62,31260741 \\
\hline 17 & 132,2926951336360 & 149,2341664240320 & 132,2926951336360 & 126,5290584181270 & 132,2926951336360 & 133,6523186474050 & 132,2926951336360 & 132,2926951336370 \\
\hline 18 & 48,9728690285128 & 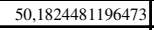 & 48,9728690285128 & 48,9881931684996 & 48,9728690285131 & 48,8318937232876 & $\begin{array}{l}48,9728690285128 \\
\end{array}$ & 48,9728690285129 \\
\hline 19 & 143,6237449146800 & 155,8361845029320 & 143,6237449146800 & 138,8073534985030 & 143,6237449146800 & 146,7613376387640 & 143,6237449146800 & 143,6237449146810 \\
\hline 20 & 35,7791821784491 & 36,5628659854903 & 35,7791821784491 & 35,6671747721295 & 35,7791821784491 & 35,4538545510061 & 35,7791821784490 & 35,7791821784493 \\
\hline 21 & 155,5355725420390 & 162,4382025818330 & 155,5355725420390 & 152,9777409127050 & 155,5355725420390 & 158,8474759730170 & 155,5355725420390 & 155,5355725420410 \\
\hline 22 & 22,6171813071349 & 22,9432838513333 & 22,6171813071350 & 22,4456776216884 & 22,6171813071351 & 22,2737799226119 & 22,6171813071352 & 22,6171813071352 \\
\hline 23 & 169,0402206607330 & 169,0402206607330 & 169,0402206607330 & 169,0402206607330 & 169,0402206607330 & 169,0402206607330 & 169,0402206607310 & 169,0402206607330 \\
\hline 24 & 9,3237017171763 & 9,3237017171763 & 9,3237017171763 & 9,3237017171763 & 9,3237017171763 & 9,3237017171763 & 9,3237017171763 & 9,3237017171763 \\
\hline
\end{tabular}

É possível observar para todos os casos testados que a condição de aderência total fibra-matriz é garantida se a ordem de aproximação da fibra é pelo menos a mesma ordem de aproximação adotada para os elementos bidimensionais. 


\section{APÊNDICE C - GERADOR DE FIBRAS LONGAS E CURTAS ALEATÓRIAS}

Neste apêndice, faz-se uma breve apresentação do gerador de fibra implementado. Nele é possível gerar malhas de fibras longas e curtas aleatórias para a análise de problemas bidimensionais reforçados com fibras e placas e casca laminadas reforçadas com fibras.

O gerador de fibras foi implementado em linguagem FORTRAN, versão VISUAL COMPAQ 6.6. Basicamente, a estrutura do programa é subdividida em três fases, o préprocessamento, o processamento e o pós-processamento. A Figura 95 mostra a estrutura do programa desenvolvido. $\mathrm{Na}$ etapa do pré-processamento, o usuário deve fornecer as características do problema para o qual se deseja gerar as fibras em um arquivo de texto mostrado posteriormente neste Apêndice. Estas informações são lidas pelo compilador e utilizadas pelas sub-rotinas de cálculo da fase de processamento para a geração das fibras. Por fim, na fase de pós-processamento, os resultados do processamento são impressos em um arquivo de texto.txt.

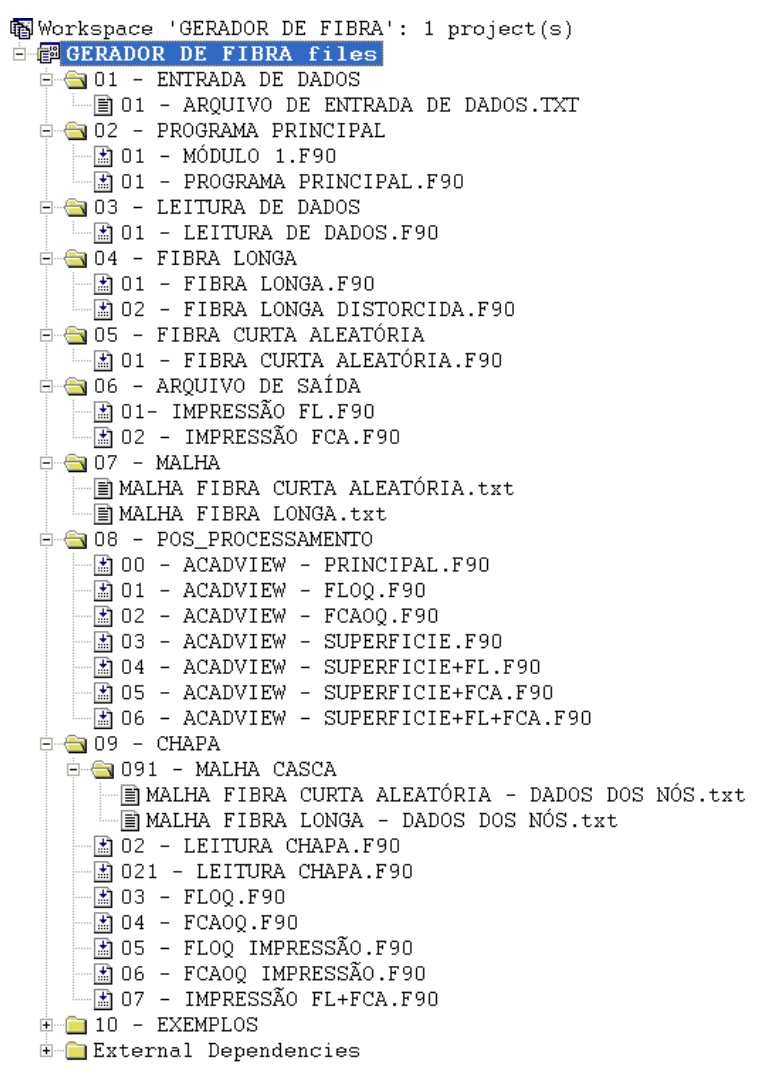

Figura 95 - Estrutura do programa para geração de fibras. 
Além disto, o código desenvolvido foi acoplado ao ACADVIEW, Software de acesso livre para pós-processamento em elementos finitos 2D e 3D, disponível para download em http://www.set.eesc.usp.br/portal/pt/softwares/27-pesquisa/softwares/157-acadview.

A estrutura do programa principal, que gerencia as sub-rotinas que constituem o programa, é mostrada na Figura 96. Nela destacam as estruturas para a geração das fibras longas, das fibras curtas aleatórias, dos dados quando estas estão contidas em uma superfície de referência informada pelo usuário e impressão dos dados gerados utilizados como arquivos de entrada para outros programas, como por exemplo, o de chapa descrito no Capítulo 4 e o de casca descrito no Capítulo 6.

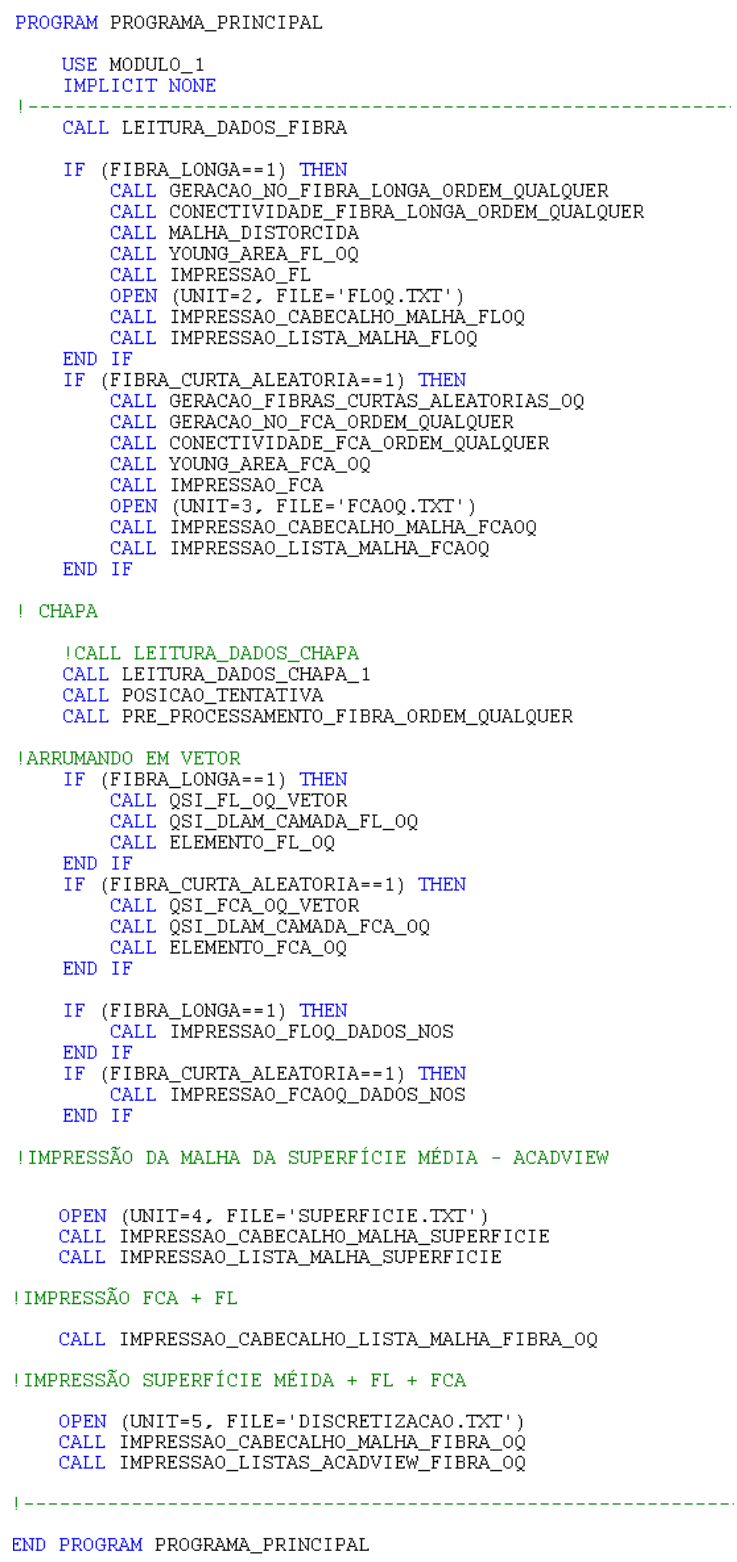

Figura 96 - Programa principal para gerenciamento das sub-rotinas de geração das fibras. 
A Figura 97 e a Figura 98 mostram, respectivamente, o arquivo de entrada de dados para a geração das fibras e o arquivo de entrada de dados da superfície de referência para a geração das coordenadas adimensionais dos nós da fibra.

No arquivo de entrada de dados das fibras, Figura 97, deve-se informar o tipo de fibra a ser gerado, se curva aleatória, longa ou ambos. Para o caso de fibras aleatórias deve-se informar o número de blocos onde serão geradas as fibras, o grau do polinômio e o número de pontos de Gauss a ser utilizado na integração numérica das fibras. Em seguida devem-se informar os nós inicial e final de cada bloco, as propriedades físicas e geométricas das fibras contidas em cada bloco e a quantidade de fibras a ser gerada.

No arquivo de entrada de dados da superfície de referência deve-se informar o número total de nós da discretização, o número de elementos, o número de nós do elemento, as coordenadas dos nós, a incidência dos elementos e o tipo de fibra considerado na discretização.

Para gerar as malhas das superfícies de referência utilizou-se um gerador pronto, disponível no GMEC/USP. O arquivo gerado por este programa é utilizado como arquivo de entrada do gerador de fibra desenvolvido, devendo-se apenas inserir as três ultimas linhas para informar o tipo de fibra a ser gerado.

Uma vez que são especificados os nós inicial e final das fibras longas, o programa subdivide estas de acordo com a quantidade especificada pelo usuário, gera a conectividade, as coordenadas adimensionais e atribui as propriedades conforme especificado no arquivo de entrada de dados.

As fibras aleatórias são geradas em um domínio, aqui designado como bloco, especificado pelo usuário. O domínio pode ser subdivido em vários blocos, de vários tamanhos, desde que limitado ao domínio do problema, e que podem conter fibras com propriedades diferentes.

Uma vez conhecidas as coordenadas iniciais e finais do(s) bloco(s), a obtenção das fibras se dá pela geração de dois pontos aleatórios com o comando interno do FORTRAN identificado como RANDOM_NUMBER(B), que retorna um número aleatório que satisfaz $0<\mathrm{B}<1$. O primeiro ponto gerado é tomado como coordenada inicial da fibra e, uma vez que o comprimento da fibra foi especificado pelo usuário, o segundo ponto gerado é utilizado como direção, cossenos diretores, da fibra.

Conhecidos os nós inicial e final da fibra faz-se uma verificação para assegurar que os pontos estão contidos no domínio do problema. Caso estejam, a próxima fibra é gerada, caso 
contrário calcula-se uma nova coordenada final até que a fibra esteja contida no bloco especificado.

Este procedimento é repetido para o número de fibras total do problema em cada bloco especificado pelo usuário.

O código computacional do procedimento descrito pode ser visualizado na Figura 99.

As demais sub-rotinas desenvolvidas são para atribuir a ordem de aproximação das variáveis da fibra especificada pelo usuário, determinar a conectividade e as demais propriedades, bem como imprimir os dados da discretização. Os arquivos de saída gerados pelo programa podem ser visualizados na Figura 100 e na Figura 101.

No arquivo de saída mostrado na Figura 100 são impressos, o número de nós e de elementos da discretização, o grau de aproximação da fibra e o número de pontos de Gauss utilizados para a integração numérica. Além disto, têm-se as coordenadas x, y e z, a conectividade, o módulo de elasticidade e a área da seção transversal de cada fibra.

Na Figura 101, tem-se as coordenadas adimensionais dos nós da fibra em relação à superfície de referência e em relação a espessura da camada, a camada onde está a fibra, a espessura da camada, a distância do centro de gravidade da lâmina considerada até a superfície de referência e em que elemento da superfície de referência está o nó de fibra considerado.

O programa imprime ainda os arquivos de dados para visualização da malha da superfície de referência, malha da fibra, e malha da superfície de referência juntamente com as fibras no ACADVIEW (Figura 102). 


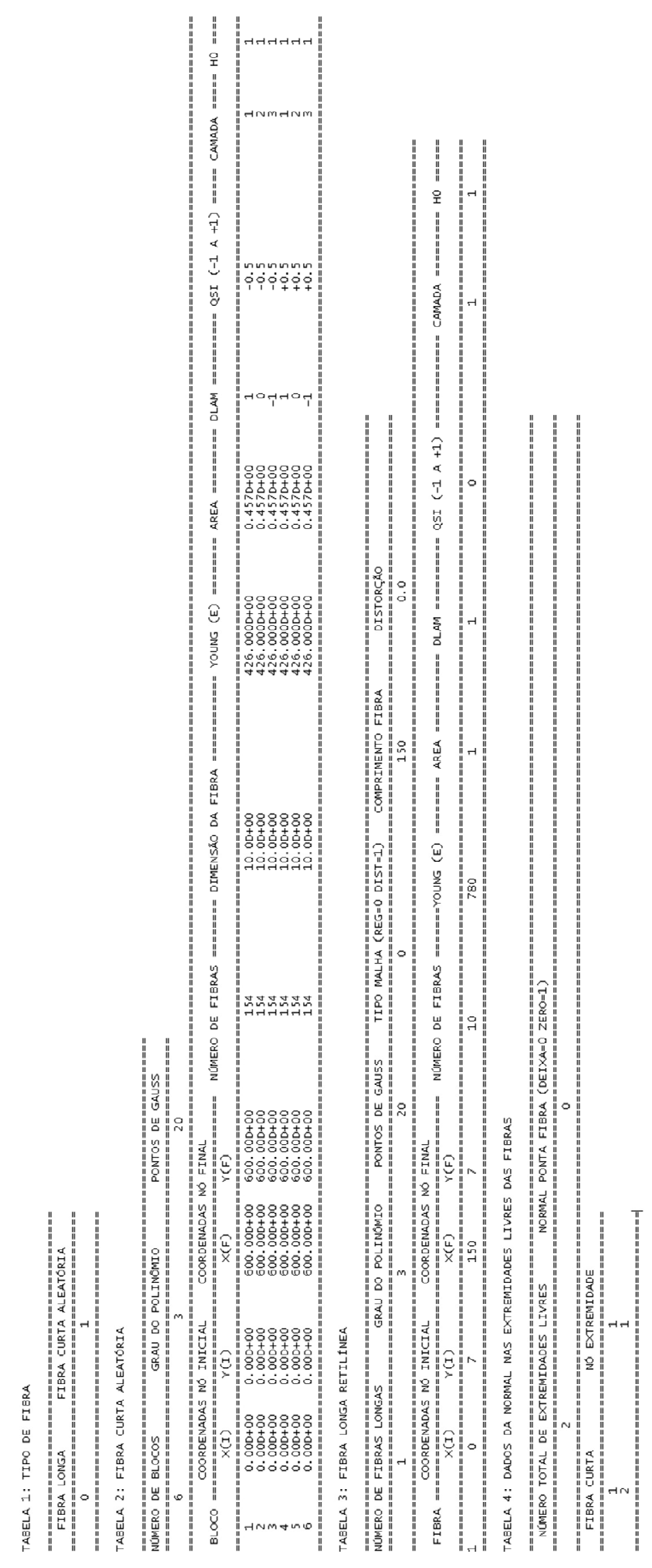

Figura 97 - Arquivo de entrada de dados para a geração das fibras. 

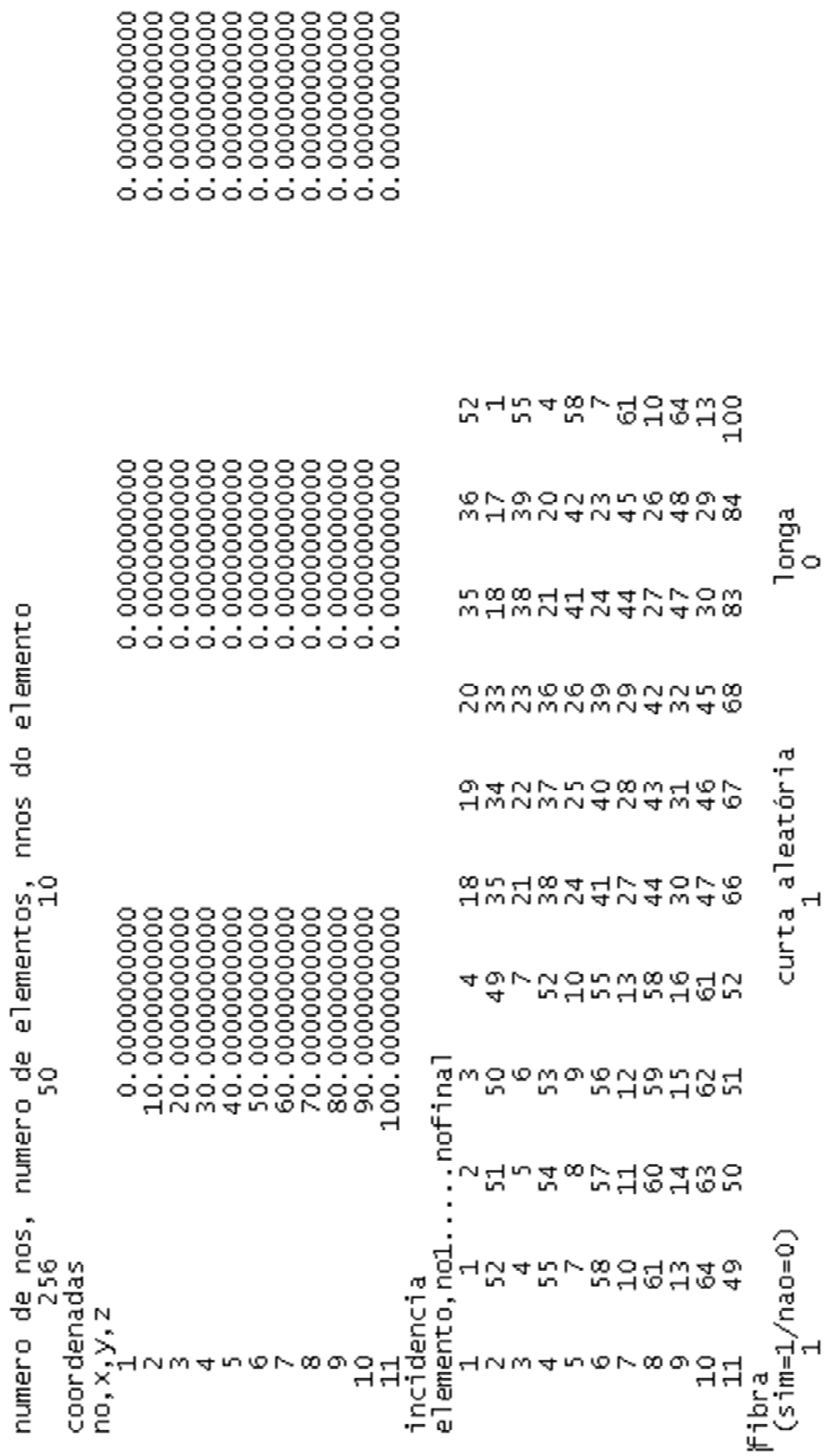

Figura 98 - Arquivo de entrada de dados da superfície de referência. 


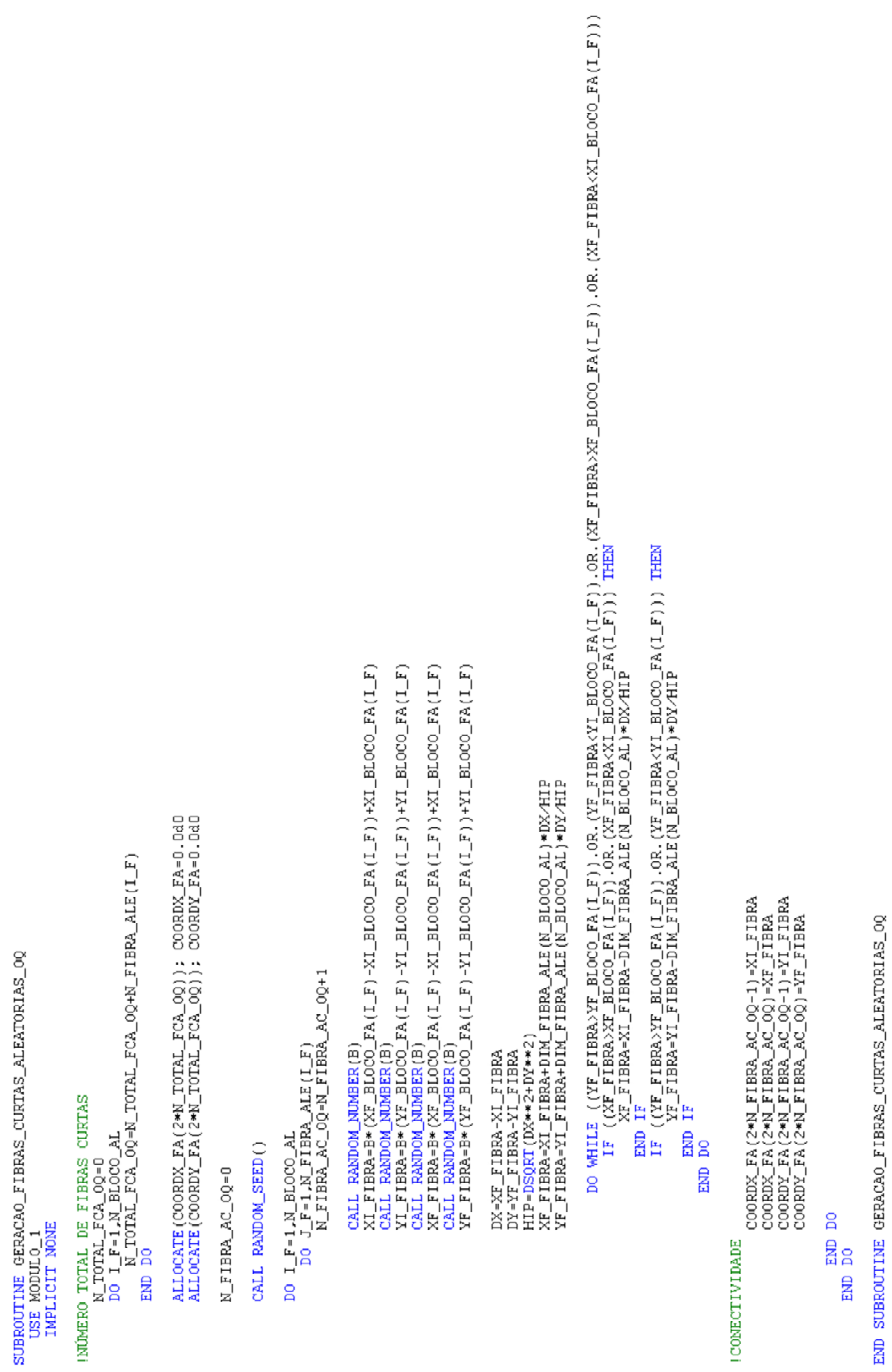

Figura 99 - Sub-rotina para geração das fibras aleatórias. 


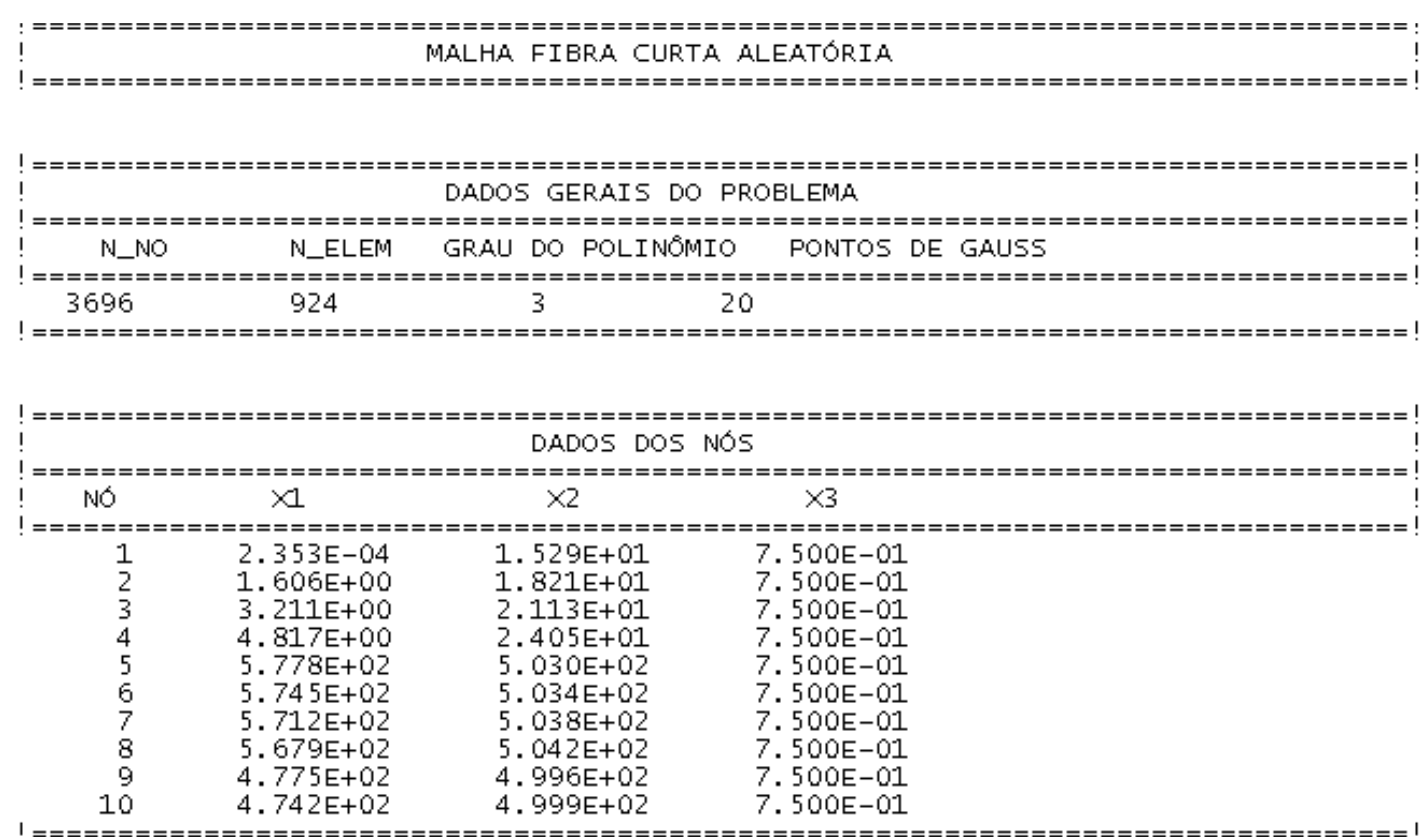

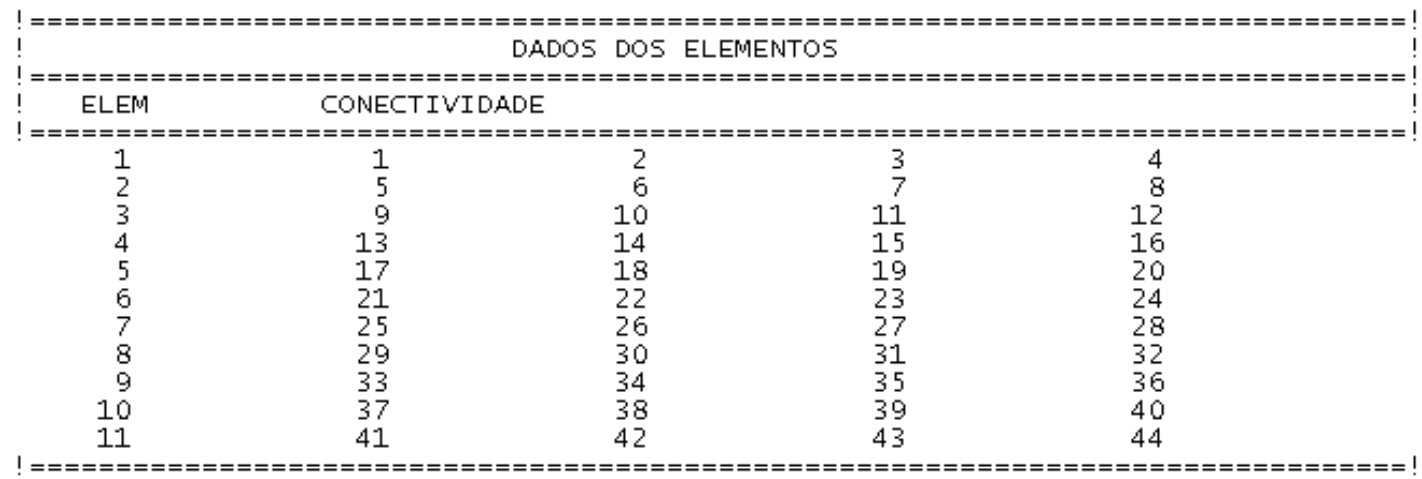

\begin{tabular}{|c|c|c|}
\hline ! & & DADOS DOS ELEMENTOS \\
\hline ! = = = = = = = & $========$ & 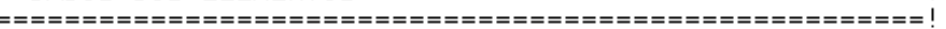 \\
\hline ELEM & M. ELAST & AREA \\
\hline$!========$ & $========$ & 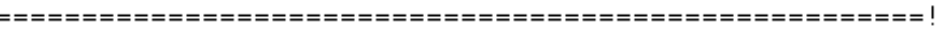 \\
\hline 1 & $4.260 \mathrm{E}+02$ & $4.570 \mathrm{E}-01$ \\
\hline 2 & $4.260 E+02$ & 4. $570 E-01$ \\
\hline 3 & $4.260 E+02$ & 4. $570 \mathrm{E}-01$ \\
\hline 4 & $4.260 \mathrm{E}+02$ & 4. $570 \mathrm{E}-01$ \\
\hline 5 & $4.260 E+02$ & 4. $570 \mathrm{E}-01$ \\
\hline 6 & $4.260 \mathrm{E}+02$ & 4. $570 \mathrm{E}-01$ \\
\hline 7 & $4.260 \mathrm{E}+02$ & 4. $570 \mathrm{OE}-01$ \\
\hline 8 & $4.260 \mathrm{E}+02$ & $4.570 \mathrm{E}-0 \mathrm{l}$ \\
\hline 9 & $4.260 \mathrm{E}+02$ & 4. $570 \mathrm{E}-01$ \\
\hline 10 & $4.260 E+02$ & 4. $570 \mathrm{E}-01$ \\
\hline
\end{tabular}

Figura 100 - Arquivo de saída com dados das fibras. 

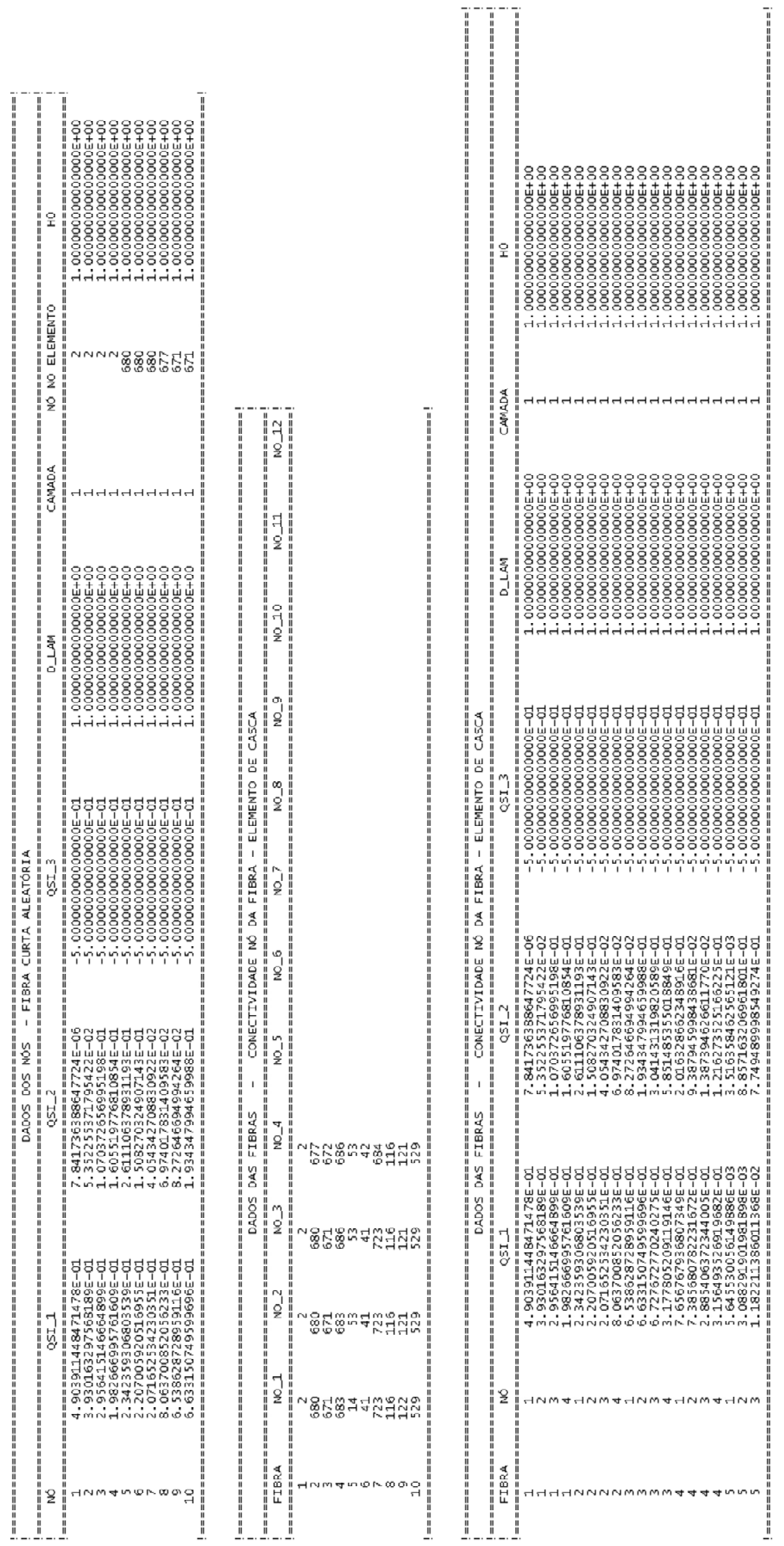

Figura 101 - Arquivo de saída com informações das lâminas. 


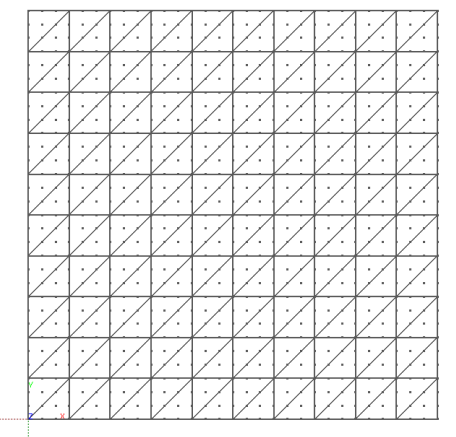

(a)

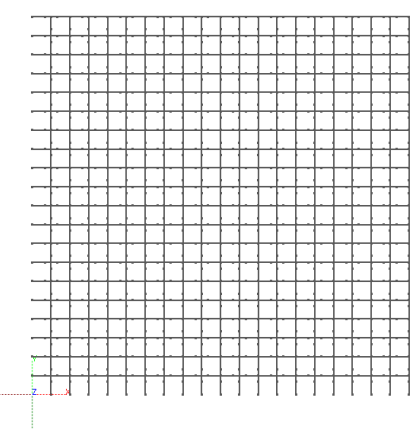

(b)

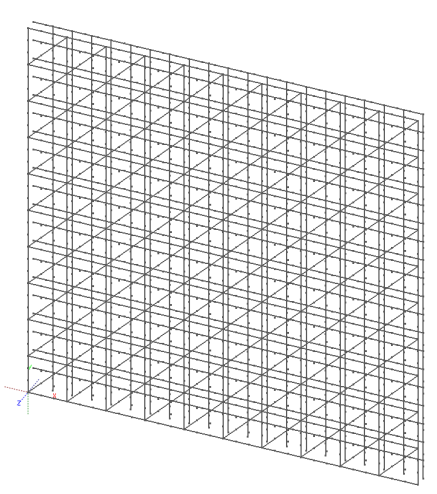

(c)

Figura 102 - Discretização obtida como o ACADVIEW: (a) superfície de referência; (b) fibras; (c) superfície de referência e fibras. 


\section{ANEXO A - POLINÔMIOS DE LAGRANGE E PRIMEIRA DERIVADA}

Seja $I$ um elemento finito unidimensional de fibra cuja ordem de aproximação é $(n-1)$, então existem $n$ funções de forma, cada uma associada a um determinado nó $l$ do elemento, que são obtidas, neste trabalho, utilizando-se os polinômios de Lagrange dados pelo produtório

$$
\phi_{l}(\xi)=\prod_{\substack{l \neq j \\ j=1}}^{n}\left(\frac{\xi-\xi_{j}}{\xi_{l}-\xi_{j}}\right)
$$

em que as coordenada adimensional $\xi_{l}$ e $\xi_{j}$ assumem valores no intervalo $[-1,+1]$ no espaço adimensional e cada função de forma $\phi_{l}(\xi)$ tem valor unitário no nó ao qual está associada e valor nulo nos demais nós do elemento.

A primeira derivada generalizada do produtório dos polinômios de Lagrange para que se possa implementar um código computacional para a análise de elementos de fibra com ordem qualquer de aproximação é dada por

$$
\frac{d}{d \xi}\left\{\phi_{l}(\xi)\right\}=\phi_{l}(\xi)\left[\sum_{\substack{l \neq j \\ j=1}}^{n} \frac{1}{\left(\xi-\xi_{j}\right)}\right] .
$$

Durante a concepção de formulações baseada no MEF para a análise de uma determinada classe de problemas podem resultar equações que, em geral, são difíceis ou que não podem ser integradas explicitamente. Neste sentido, recorre-se a uma estratégia de integração numérica para operacionalizar as integrações necessárias e assim conferir maior generalidade aos códigos computacionais desenvolvidos. Neste trabalho, utiliza-se a Quadratura de Gauss-Legendre para efetuar as integrações numéricas em domínios unidimensionais. 


\section{ANEXO B - INTEGRAÇÃO NUMÉRICA EM DOMÍNIOS TRIANGULARES}

\section{B.1. Quadratura de Hammer}

A integral na área do elemento finito triangular com coordenadas homogêneas mostrado na Figura 103 pode ser representada como

$$
\int_{A} f(x, y) d A=\int_{0}^{l} \int_{0}^{l-\xi_{2}} g\left(\xi_{1}, \xi_{2}\right) \operatorname{det} J d \xi_{1} d \xi_{2}=\sum_{i=1}^{N P H} g\left(\xi_{1}, \xi_{2}\right)_{i} w_{i} \operatorname{det} J
$$

sendo $\xi_{3}=1-\xi_{1}-\xi_{2}$ e $J$ o Jacobiano da transformação.

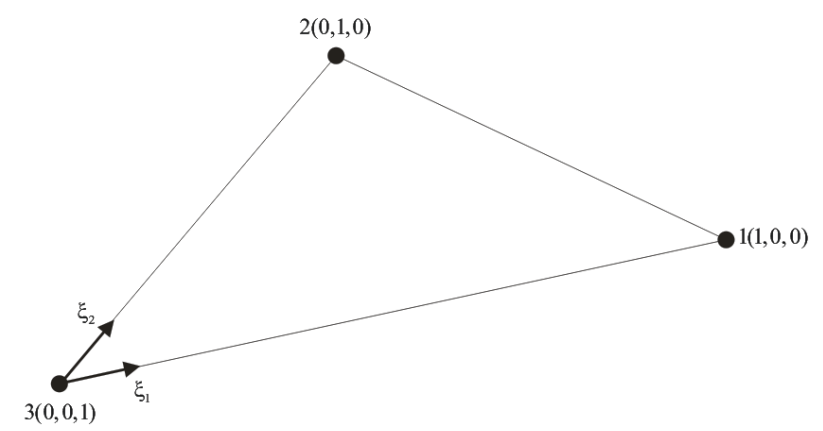

Figura 103 - Coordenas homogêneas do elemento finito triangular. (Fonte: ASSAN, 2003).

\section{B.2. Pontos e pesos de Hammer}

A Tabela 20 mostra os pontos e pesos de integração de Hammer para 1, 3, 4 e 7 pontos de integração dispostos conforme mostra a Figura 104. Esta tabela foi implementada no programa de chapa de ordem qualquer.

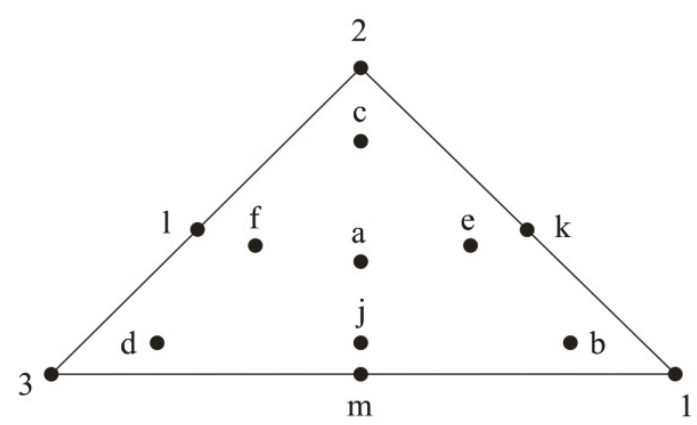

Figura 104 - Pontos de integração (Fonte: ASSAN, 2003). 
Tabela 20 - Pontos e pesos de Hammer (Fonte: ASSAN, 2003).

\begin{tabular}{|c|c|c|c|c|c|}
\hline \multirow{2}{*}{$\mathbf{N}$} & \multirow{2}{*}{ PONTOS } & \multicolumn{3}{|c|}{ COORDENADAS } & \multirow{2}{*}{$\begin{array}{c}\text { PESO } \\
w_{i}\end{array}$} \\
\hline & & $\xi_{1}$ & $\xi_{2}$ & $\xi_{3}$ & \\
\hline 1 & $\mathbf{a}$ & 0 & $1 / 3$ & $1 / 3$ & $1 / 3$ \\
\hline \multirow{3}{*}{3} & l & 0 & $1 / 2$ & $1 / 2$ & \multirow{3}{*}{$1 / 6$} \\
\hline & $\mathbf{m}$ & $1 / 2$ & 0 & $1 / 2$ & \\
\hline & $\mathbf{k}$ & $1 / 2$ & $1 / 2$ & 0 & \\
\hline \multirow{3}{*}{3} & b & $2 / 3$ & $1 / 6$ & $1 / 6$ & \multirow{3}{*}{$1 / 6$} \\
\hline & c & $1 / 6$ & $2 / 3$ & $1 / 6$ & \\
\hline & d & $1 / 6$ & $1 / 6$ & $2 / 3$ & \\
\hline \multirow{4}{*}{4} & $\mathbf{a}$ & $1 / 3$ & $1 / 3$ & $1 / 3$ & $-9 / 32$ \\
\hline & b & $3 / 5$ & $1 / 5$ & $1 / 5$ & $25 / 96$ \\
\hline & c & $1 / 5$ & $3 / 5$ & $1 / 5$ & $25 / 96$ \\
\hline & d & $1 / 5$ & $1 / 5$ & $3 / 5$ & $25 / 96$ \\
\hline \multirow{7}{*}{7} & $\mathbf{a}$ & $1 / 3$ & $1 / 3$ & $1 / 3$ & 0,1125000 \\
\hline & b & 0,7974270 & 0,1012865 & 0,1012865 & 0,0629696 \\
\hline & c & 0,1012865 & 0,7974270 & 0,1012865 & 0,0629696 \\
\hline & d & 0,1012865 & 0,1012865 & 0,7974270 & 0,0629696 \\
\hline & e & 0,4701420 & 0,4701420 & 0,0597160 & 0,0661971 \\
\hline & f & 0,0597160 & 0,4701420 & 0,4701420 & 0,0661971 \\
\hline & g & 0,4701420 & 0,0597160 & 0,4701420 & 0,0661971 \\
\hline
\end{tabular}

Na Tabela 20, N é o número de pontos de integração que pode ser associado ao grau de aproximação como: 1 ponto = aproximação linear; 3 pontos = aproximação quadrática; 4 pontos $=$ aproximação cúbica e 7 pontos $=$ aproximação quíntupla. 


\section{ANEXO C - OBTENÇÃO DAS COORDENADAS ADIMENSIONAIS DE UM PONTO CONTIDO EM UM ELEMENTO FINITO TRIANGULAR}

Neste anexo, descreve-se o procedimento para encontrar as coordenadas adimensionais de um ponto qualquer inserido em um elemento finito triangular, cujas coordenadas cartesianas dos nós são conhecidas. Este procedimento é utilizado neste trabalho para a determinação das coordenadas adimensionais dos nós do elemento finito de fibra e já foi utilizando anteriormente por (CODA, 1990; VANALLI, 2004).

A Figura 105 mostra um ponto $P$ com coordenadas cartesianas $X_{1}^{P}$ e $X_{2}^{P}$ inserido em um elemento finito triangular de área $A$.

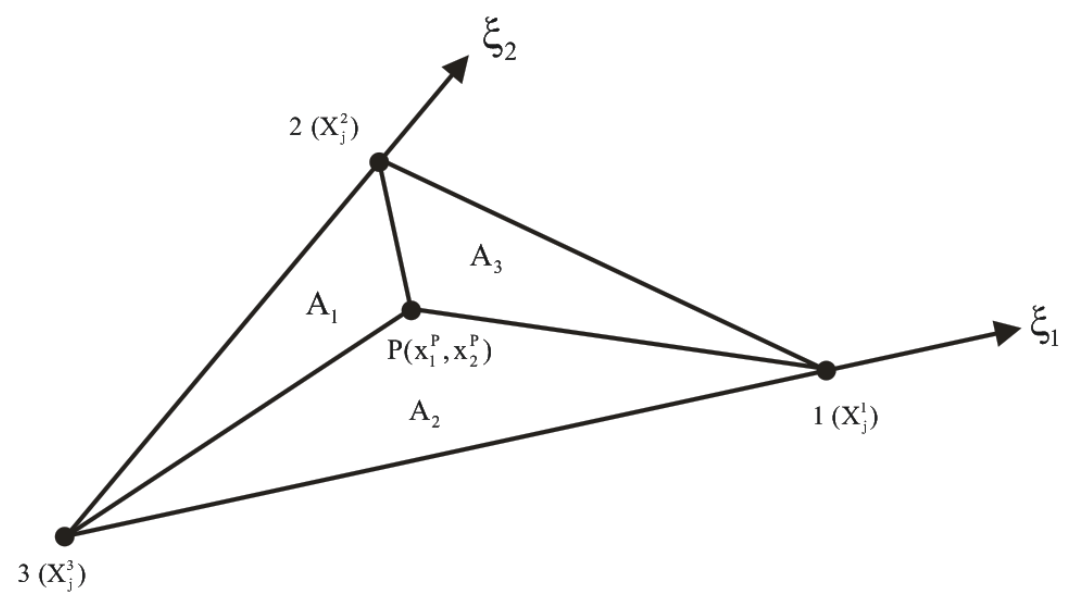

Figura 105 - PPonto P inserido em um elemento finito triangular.

As coordenadas cartesianas $X_{1}^{P}$ e $X_{2}^{P}$ do ponto $\mathrm{P}$ podem ser obtidas em função das coordenadas cartesianas dos nós do elemento finito triangular e das funções de forma associadas a estes nós calculadas no ponto P pela expressão

$$
X_{j}^{P}=\xi_{1}^{P} X_{j}^{1}+\xi_{2}^{P} X_{j}^{2}+\xi_{3}^{P} X_{j}^{3}, \quad(j=1,2)
$$

As coordenadas adimensionais $\xi_{\alpha}^{P}$ do ponto $\mathrm{P}$ podem ser obtidas pela expressão

$$
\xi_{\alpha}^{P}=\frac{1}{2 A}\left(2 A_{0}^{\alpha}+b^{\alpha} X_{1}^{P}+a^{\alpha} X_{2}^{P}\right),
$$


sendo

$$
\begin{gathered}
a^{\alpha}=X_{1}^{k}-X_{1}^{m}, \\
b^{\alpha}=X_{2}^{m}-X_{2}^{k}, \\
2 A_{0}^{\alpha}=X_{1}^{m} X_{2}^{k}-X_{1}^{k} X_{2}^{m}, \\
A=\frac{1}{2}\left(b^{1} a^{2}-b^{2} a^{1}\right) .
\end{gathered}
$$

As variáveis $\alpha, m$ e $k$ respeitam a relação cíclica

$$
\alpha=1,2,3 ; \quad m=2,3,1 ; \quad k=3,1,2,
$$

ou seja, se $\alpha=1 \quad m=2$ e $k=3$. 


\section{ANEXO D - FUNÇÕES DE FORMA DO ELEMENTO FINITO TRIANGULAR COM DEZ NÓS}

A Figura 106 mostra um elemento finito triangular com 10 nós, ou aproximação cúbica para as variáveis. Neste trabalho, este elemento é utilizado para discretizar a superfície média do elemento finito de casca. Neste sentido, utiliza-se quadratura de Hammer (ANEXO B) para integrar a superfície do elemento e quadratura de Gauss para efetuar a integração ao longo da espessura.

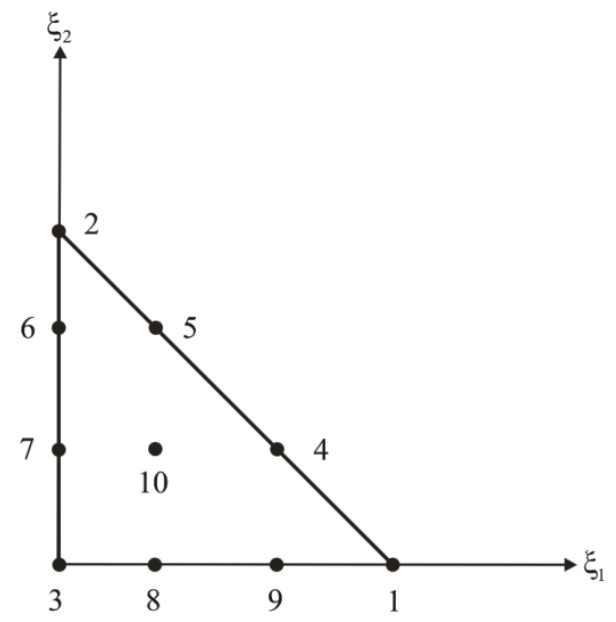

Figura 106 - Elemento finito triangular com dez nós.

\section{D.1. Funções de forma}

As funções de forma para um elemento finito triangular com dez nós (Figura 106), ou aproximação cúbica (ASSAN, 2003) são dadas, respectivamente, por

$$
\begin{gathered}
\phi_{1}=\frac{1}{2} \xi_{1}\left(3 \xi_{1}-1\right)\left(3 \xi_{1}-2\right), \phi_{2}=\frac{1}{2} \xi_{2}\left(3 \xi_{2}-1\right)\left(3 \xi_{2}-2\right), \\
\phi_{3}=\frac{1}{2} \xi_{3}\left(3 \xi_{3}-1\right)\left(3 \xi_{3}-2\right), \phi_{4}=\frac{9}{2} \xi_{1} \xi_{2}\left(3 \xi_{1}-1\right), \\
\phi_{5}=\frac{9}{2} \xi_{1} \xi_{2}\left(3 \xi_{2}-1\right), \phi_{6}=\frac{9}{2} \xi_{2} \xi_{2}\left(3 \xi_{2}-1\right), \\
\phi_{7}=\frac{9}{2} \xi_{2} \xi_{3}\left(3 \xi_{3}-1\right), \phi_{8}=\frac{9}{2} \xi_{3} \xi_{1}\left(3 \xi_{3}-1\right),
\end{gathered}
$$




$$
\phi_{9}=\frac{9}{2} \xi_{3} \xi_{1}\left(3 \xi_{1}-1\right), \phi_{10}=27 \xi_{1} \xi_{2} \xi_{3}
$$

\section{D.2. Primeira derivada das funções de forma}

As derivadas das funções de forma apresentadas no item D1 em relação às coordenadas adimensionais $\xi_{1}$ e $\xi_{2}$ são

$$
\begin{aligned}
& \frac{\partial \phi_{1}}{\partial \xi_{1}}=\frac{27}{2} \xi_{1}^{2}-9 \xi_{1}+1, \frac{\partial \phi_{1}}{\partial \xi_{2}}=0 \\
& \frac{\partial \phi_{2}}{\partial \xi_{1}}=0, \frac{\partial \phi_{2}}{\partial \xi_{2}}=\frac{27}{2} \xi_{2}^{2}-9 \xi_{2}+1 \\
& \frac{\partial \phi_{3}}{\partial \xi_{1}}=-\frac{11}{2}+18 \xi_{1}+18 \xi_{2}-\frac{27}{2} \xi_{1}^{2}-27 \xi_{1} \xi_{2}-\frac{27}{2} \xi_{2}^{2}, \\
& \frac{\partial \phi_{3}}{\partial \xi_{2}}=-\frac{11}{2}+18 \xi_{1}+18 \xi_{2}-\frac{27}{2} \xi_{1}^{2}-27 \xi_{1} \xi_{2}-\frac{27}{2} \xi_{2}^{2}, \\
& \frac{\partial \phi_{4}}{\partial \xi_{1}}=27 \xi_{1} \xi_{2}-\frac{9}{2} \xi_{2}, \frac{\partial \phi_{4}}{\partial \xi_{2}}=\frac{9}{2} \xi_{1}\left(3 \xi_{1}-1\right), \\
& \frac{\partial \phi_{5}}{\partial \xi_{1}}=27 \xi_{1} \xi_{2}-\frac{9}{2} \xi_{1}, \frac{\partial \phi_{5}}{\partial \xi_{2}}=\frac{9}{2} \xi_{2}\left(3 \xi_{2}-1\right), \\
& \frac{\partial \phi_{6}}{\partial \xi_{1}}=-\frac{9}{2} \xi_{2}\left(3 \xi_{2}-1\right), \frac{\partial \phi_{6}}{\partial \xi_{2}}=36 \xi_{2}-\frac{9}{2}-27 \xi_{1} \xi_{2}+\frac{9}{2} \xi_{1}-\frac{81}{2} \xi_{2}^{2}, \\
& \frac{\partial \phi_{7}}{\partial \xi_{1}}=-\frac{45}{2} \xi_{2}+27 \xi_{1} \xi_{2}+27 \xi_{2}^{2}, \frac{\partial \phi_{7}}{\partial \xi_{2}}=9-\frac{45}{2} \xi_{2}-45 \xi_{2}+\frac{27}{2} \xi_{1}^{2}+54 \xi_{1} \xi_{2}+\frac{81}{2} \xi_{2}^{2}, \\
& \frac{\partial \phi_{8}}{\partial \xi_{1}}=-45 \xi_{1}+\frac{81}{2} \xi_{1}^{2}+54 \xi_{1} \xi_{2}+9-\frac{45}{2} \xi_{2}+\frac{27}{2} \xi_{2}^{2}, \frac{\partial \phi_{8}}{\partial \xi_{2}}=-\frac{45}{2} \xi_{1}+27 \xi_{1}^{2}+27 \xi_{1} \xi_{2}, \\
& \frac{\partial \phi_{9}}{\partial \xi_{1}}=-\frac{81}{2} \xi_{1}^{2}+36 \xi_{1}-\frac{9}{2}-27 \xi_{1} \xi_{2}+\frac{9}{2} \xi_{2}, \frac{\partial \phi_{9}}{\partial \xi_{2}}=-\frac{9}{2} \xi_{1}\left(3 \xi_{1}-1\right), \\
& \frac{\partial \phi_{10}}{\partial \xi_{1}}=27 \xi_{2}-54 \xi_{1} \xi_{2}-27 \xi_{2}^{2}, \frac{\partial \phi_{10}}{\partial \xi_{2}}=27 \xi_{1}-27 \xi_{1}^{2}-54 \xi_{1} \xi_{2} .
\end{aligned}
$$

